\title{
Materials for Electrochemical Energy Conversion and Storage
}

\author{
by \\ Molly M. MacInnes \\ A dissertation submitted in partial fulfillment \\ of the requirements for the degree of \\ Doctor of Philosophy \\ (Chemistry) \\ in the University of Michigan \\ 2020
}

Doctoral Committee:

Professor Nicolai Lehnert, Co-Chair

Professor Stephen Maldonado, Co-Chair

Professor Zhan Chen

Assistant Professor Charles McCrory

Assistant Professor Nirala Singh 
Molly M. MacInnes

mmacinne@umich.edu

ORCID iD: 0000-0001-9513-7402

(C) Molly M. MacInnes 2020 


\section{Dedication}

To my parents, Ian and Michele, and to my sister and brother, Corinne and Joe. 


\section{Acknowledgements}

I would first like to acknowledge the support and mentorship of my two advisors, Professor Nicolai Lehnert and Professor Stephen Maldonado. Through your guidance I have grown as a scientist and as a professional. I learned a great deal about mentorship from both of you. Furthermore, I want to thank you for your support of my professional development and outreach goals, without which my time in graduate school would have had much less meaning.

To the members of the Lehnert lab and the Maldonado lab, past and present: thank you for always being welcoming, supportive, and non-judgmental (except where it was necessary). I will miss the banter and sarcasm that pervaded the Maldonado lab, and I will miss the long road trips and occasional board games with the Lehnert lab and the camaraderie and antics we had at conferences together. In particular I want to thank Dr. Shawn Eady, who was my very first mentor in graduate school. Shawn is one of the kindest and most patient people I know, not to mention a fantastic researcher. Shawn taught me the first things I knew about electrochemistry and had the patience to remind me how a Schlenk line works, among other basic laboratory skills that I had forgotten. When I think of people who are able to coolly proceed in the face of a mountain of responsibilities, I think of Shawn, and through my graduate career I have tried to emulate that ability. Furthermore, I want to thank the three people in the Maldonado lab who have been the biggest mentors to me over the years. Dr. Susu Lee, Dr. Josh Demuth, and Dr. Sofiya Hlynchuk. Susu and Josh seemed to have a vast well of knowledge between the two of them that I could always pick at. I learned a great deal from both of them and they were always willing to talk and bounce around ideas with me. Sofiya was with me for most of my time in graduate school. She was someone I always felt comfortable coming to with a question, whether it was about research, school, or personal problems. Sofiya made my time in grad school so much more enjoyable than it would have been without her and her absence this past year has been difficult.

I want to thank the staff in the chemistry department and elsewhere at the University of Michigan. In particular I want to thank Liz Oxford, Katie Foster, Roy Wentz, Tracy Stevenson, 
and the staff at $(\mathrm{MC})^{2}$ for always being positive and welcoming, excelling at their jobs, and generally making all of our lives easier.

All my friends, both in Ann Arbor and scattered across the rest of the world have been of the utmost importance to me over the past five years. I am so grateful to have so many generous and loving people to share in my successes and comfort me in my failures. There are too many names that I would like to list here, so instead I just want to say thank you to you all.

I want to thank all the professors who taught me at Oberlin College, especially Professor Rebecca Whelan, my former academic adviser, and the late Professor Jesse Rowsell, my former research advisor who mentored me for my honors thesis project. Additionally, I want to thank Dr. Stosh Kozimor and Kevin Boland who were my mentors in my very first research experience during a summer internship at the Los Alamos National Lab. It was during this summer that I discovered a passion for scientific research and that is partly thanks to both of them.

And lastly, I want to thank my family. Without you I would be nowhere near where I am now. I have received nothing but love and support from all of you. I'm so happy I could spend the last five years living within the same state as at least three of you, and the last two years living within the same walls as one of you. Thank you so much for everything you have done for me, for your unconditional love and support. I love you all so much. 


\section{Table of Contents}

$\begin{array}{ll}\text { Dedication } & \text { ii }\end{array}$

$\begin{array}{ll}\text { Acknowledgements } & \text { iii }\end{array}$

List of Tables $\quad$ vii

List of Figures

$\begin{array}{ll}\text { Abstract } & \text { xvi }\end{array}$

$\begin{array}{ll}\text { Chapter 1. Introduction } & 1\end{array}$

1.1 A societal need for clean energy 1

1.2 Photoelectrochemical cells for solar energy conversion. 5

$\begin{array}{ll}1.3 \text { Heterogeneous molecular electrocatalysis. } & 7\end{array}$

1.4 Reduced graphene oxide. 14

1.6 Other redox species for electrochemical energy storage $\quad 21$

1.7 Scope of thesis $\quad 22$

Chapter 2. Reduction of Graphene Oxide Thin Films by Cobaltocene and

$\begin{array}{ll}\text { Decamethylcobaltocene } & 24\end{array}$

2.1 Introduction 24

2.2 Experimental. 27

2.3 Results. $\quad 31$

2.4 Discussion $\quad 44$

2.5 Conclusions. $\quad 48$

Chapter 3. Physisorption of a Molecular Proton Reduction Electrocatalyst on Thin Graphitic

$\begin{array}{lr}\text { Films } & 50\end{array}$

$\begin{array}{ll}3.1 \text { Introduction } & 50\end{array}$ 
$\begin{array}{ll}3.2 \text { Experimental } & 51\end{array}$

3.3 Results $\quad 54$

$\begin{array}{ll}3.4 \text { Discussion } & 59\end{array}$

$\begin{array}{ll}3.5 \text { Conclusions } & 62\end{array}$

Chapter 4. Reduced Graphene Oxide Thin Films Embedded with a Molecular Catalyst: Kinetic

$\begin{array}{ll}\text { Considerations } & 64\end{array}$

4.1 Introduction $\quad 64$

$\begin{array}{ll}4.2 \text { Experimental } & 66\end{array}$

$\begin{array}{ll}4.3 \text { Results } & 69\end{array}$

$\begin{array}{ll}4.5 \text { Conclusions } & 79\end{array}$

Chapter 5. Discovery of an Unusually Stable Reduced Viologen via Synergistic Folding and

$\begin{array}{lr}\text { Encapsulation } & 80\end{array}$

$\begin{array}{ll}5.1 \text { Introduction } & 80\end{array}$

$\begin{array}{ll}5.2 \text { Experimental. } & 82\end{array}$

$\begin{array}{ll}5.3 \text { Results. } & 86\end{array}$

$\begin{array}{ll}5.4 \text { Discussion. } & 98\end{array}$

$\begin{array}{ll}5.5 \text { Conclusions. } & 104\end{array}$

Chapter 6. Future Work 106

6.1 Covalent grafting small molecules to RGO thin films 106

6.2 In situ x-ray absorption measurements on adsorbed cobalt bis(dichlorobenzenedithiolate) 112

6.3 Heterogeneous photoelectrocatalysis using p-GaP photoelectrodes with an electrocatalyst adsorbed to an RGO thin film 118

6.4 Passivation of n-Si photoelectrodes with RGO thin films 123

6.5 Graphene oxide self-assembled monolayers on silicon oxide surfaces 128

Chapter 7. Summary and Conclusions 131

$\begin{array}{ll}\text { 8. Bibliography } & 138\end{array}$ 


\section{List of Tables}

Table 1.1 Reported molecular proton reduction catalysts immobilized on inert electrodes........ 11

Table 1.2 Reported molecular proton reduction catalysts immobilized on semiconductor

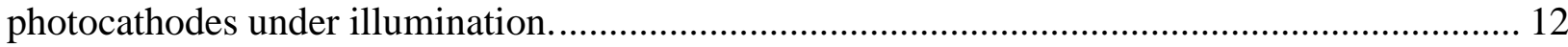

Table 2.1 Reported methods for the preparation of reduced graphene oxide and the resulting

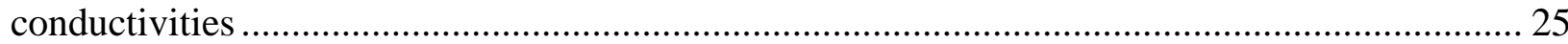

Table 2.2 Resistance and conductivities of GO and RGO films prepared under different conditions, measured by four-point probe.

Table 2.3 X-ray Photoelectron C 1s Spectra Fitting Results for As-prepared and Reduced Graphene Oxide Films ${ }^{a}$

Table 2.4 Raman Spectra for As-prepared and Reduced Graphene Oxide Films ${ }^{a, b}$

Table 5.1 Voltammetric features for 2, 3, and MV alone and in the presence of cucurbiturils as shown in Figure 5.1 


\section{List of Figures}

Figure 1.1 Three methods for solar water splitting: (a) electrolysis of water driven by an external photovoltaic, (b) photoelectrochemical water splitting, (c) photochemical water splitting. .......... 3

Figure 1.2 Example scheme of solar energy converted first (a) through the redox reaction A $\rightarrow$ $\mathrm{A}^{-}$followed by (b) conversion to $\mathrm{H}_{2}$ by electron transfer through a proton reduction catalyst. ..... 5

Figure 1.3 Example setup of a water-splitting electrolysis cell................................................ 8

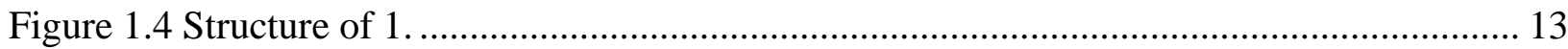

Figure 1.5 Structures of graphene (left) and RGO (right). ....................................................... 15

Figure 1.6 Scheme showing the synthetic steps to obtain RGO from graphite powder. Double

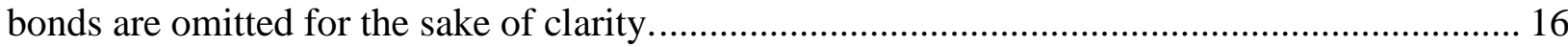

Figure 1.7 Sample set of XP C1s spectra of GO (top), RGO (middle), and graphite (bottom) that have been fitted with a series of Gaussian-Lorentzian peaks.

Figure 1.8 Sample Raman spectra of GO (top), RGO (middle), and graphite (bottom). The D, G, and G' bands are indicated. A $785 \mathrm{~nm}$ Raman laser excitation was used to collect these spectra.

Figure 1.9 Sample AFM images of (a) individual sheets of GO and (b) a thicker film of RGO on

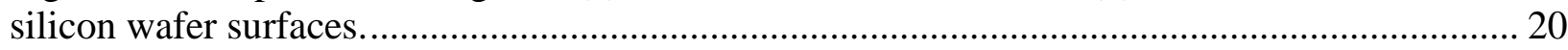

Figure 1.10 Encapsulation of methyl viologen radicals by cucurbit[7]uril (left) and cucurbit[8]uril (right).

Figure 2.1 Atomic force microscopy (AFM) images and height profiles of representative GO and RGO films used for four-point probe resistivity measurements. AFM image of (a) a GO film on glass, (c) a GO film treated with cobaltocene \& TFA on glass, (e) a GO film treated with decamethylcobaltocene on glass, and (g) a GO film treated with just TFA on glass. Height profiles along the lines denoted as 1 are shown in (b), (d), (f), and (h), respectively. Scale bars: 2 $\mu \mathrm{m}$.

Figure 2.2 a) Photograph of (I) a clean glass microscope slide; (2) a GO film deposited on a glass slide; (3) a GO film on glass treated with cobaltocene; (4) a GO film on glass treated with cobaltocene \& TFA; and (5) a GO film on glass treated with decamethylcobaltocene. b) Corresponding transmittance spectra of the films $2-5$ in (a). Scale bar: $1 \mathrm{~cm}$. 
Figure 2.3 (Top) Absorption spectra of cobaltocene in acetonitrile before (black line) and after (red line) addition of one molar equivalent of trifluoroacetic acid. (Bottom) Absorption spectra of cobaltocenium in acetonitrile.

Figure 2.4 Representative high-resolution C 1s X-ray photoelectron spectra of an as-cast GO film and after 30 minute immersions in solutions of cobaltocene, cobaltocene \& TFA, and decamethylcobaltocene, respectively. Thin vertical dashed lines are guides for the eye indicating the binding energies used for the five individual peaks (blue, purple, orange, green, and violet lines) used to generate the fit (solid red line). The backgrounds used to generate the fits are shown as thick dashed lines.

Figure 2.5 Representative Co 2p XP spectra of RGO films treated with cobaltocene \& TFA (top) and films treated with decamethylcobaltocene (bottom). The black lines correspond to freshly prepared RGO films and the red lines correspond to the same films after soaking in the respective solvents for several days. The solvents used for this removal of cobalt species was acidified acetonitrile in the case of films treated with cobaltocene \& TFA and dichloromethane in the case of films treated with decamethylcobaltocene....................................................... 36

Figure 2.6 Representative energy dispersive x-ray spectra (EDS) of an as-cast GO film on silicon and after 30 minute immersions in solutions of cobaltocene, cobaltocene \& TFA, and decamethylcobaltocene, respectively. 37

Figure 2.7 Representative Raman spectra of an as-cast GO film and after 30 minute immersions in solutions of cobaltocene, cobaltocene \& TFA, and decamethylcobaltocene, respectively. All raw spectra were normalized to the intensity of the peak near $1320 \mathrm{~cm}^{-1}$. The intensity of the spectra between 2200 and $3200 \mathrm{~cm}^{-1}$ has been increased by an order of magnitude to better visualize the peaks in this region. The scale bar highlights the intensity above the baseline of the overlap region between the $D$ and $G$ peaks.

Figure 2.8 Contact mode atomic force micrographs of (a) an as-cast GO film and after 30 minute immersions in solutions of (b) cobaltocene, (c) cobaltocene \& TFA, and (d) decamethylcobaltocene, respectively. Scale bars: $2 \mu \mathrm{m}$.

Figure 2.9 a, c) GO films deposited on indium thin films evaporated onto glass. b) The film in (a) after immersion in 50\% HI solution for 30 seconds. d) The film in (c) after treatment with cobaltocene \& TFA. Scale bar: $1 \mathrm{~cm}$.

Figure 2.10 (a) and (c) GO films patterned on paper. (b) The film in (a) after immersion in 50\% HI solution for 30 seconds. (d) The film in (c) after treatment with cobaltocene \& TFA. Scale bar: $1 \mathrm{~cm}$

Figure 2.11 Absorption spectra and cyclic voltammetric responses of GO and RGO films after immersion in $50 \mathrm{mM}$ methanol solutions for 15 minutes followed by rinsing with neat methanol. a) Absorption spectrum for crystal violet adsorbed on a GO film and b) the corresponding voltammetric responses before and after crystal violet adsorption. c) Absorption spectrum for crystal violet adsorbed on an RGO film prepared by reduction with cobaltocene and d) the corresponding voltammetric responses for the RGO film before and after crystal violet adsorption. e) Absorption spectrum for crystal violet adsorbed on an RGO film prepared by 
reduction with cobaltocene and TFA and f) the corresponding voltammetric responses for the RGO film type before and after crystal violet adsorption. g) Absorption spectrum for crystal violet adsorbed on an RGO film prepared by reduction with decamethylcobaltocene and $h$ ) the corresponding voltammetric responses for the RGO film type before and after crystal violet adsorption. Voltammetric responses were recorded in acetonitrile containing $0.1 \mathrm{M}$ tetrabutylammonium perchlorate and $40 \mathrm{mM}$ TFA. Scan rate: $10 \mathrm{mV} \mathrm{s}^{-1}$.

Figure 3.1 Co 2p XP spectra of RGO (blue), MWCNT (red), and graphene (black) after soaking in a $5 \mathrm{mM}$ solution of 1 .

Figure 3.2 Cyclic voltammetry of various graphitic thin films after soaking in a $5 \mathrm{mM}$ solution of 1 in acetonitrile for $>1 \mathrm{hr}$ with (top) and without (bottom) a Nafion capping layer. Supporting electrolyte was deaerated $0.1 \mathrm{M} \mathrm{KPF}_{6}$ (black), with $2 \mathrm{mM}$ TFA (red), $4 \mathrm{mM}$ TFA (blue), $6 \mathrm{mM}$ TFA (green), or $8 \mathrm{mM}$ TFA (orange). The dashed black curves are graphitic surfaces with no 1 adsorbed in $8 \mathrm{mM}$ TFA. The vertical dashed lines are guides for the eye to show shift in onset of current with a Nafion capping layer. The scan rate was $50 \mathrm{mV} / \mathrm{s}$.

Figure 3.3 Repeated voltammetric cycling of an fd-RGO film that had been soaked in a $5 \mathrm{mM}$ solution of 1 in acetonitrile for $>1 \mathrm{hr}$. Supporting electrolyte was deaerated $0.1 \mathrm{M} \mathrm{KPF}_{6}$ with 10 $\mathrm{mM}$ TFA (left) and $50 \mathrm{mM}$ TFA (right). The scan rate was $50 \mathrm{mV} / \mathrm{s}$.

Figure 3.4 Atomic force micrographs (left) and optical photographs (right) of a dropcasted GO thin film (top), a polished graphite rod (middle), and a freeze-dried GO film (bottom). The RMS roughness values calculated for each of these materials are $12 \mathrm{~nm}$ for GO, $104 \mathrm{~nm}$ for graphite, and $262 \mathrm{~nm}$ for fd-RGO.

Figure 3.5 Scanning electron micrographs of a freeze-dried GO film on a silicon wafer. Cross section view is on the left. The middle and right images are top down views at different magnifications.

Figure 3.6 AFM images of Nafion thin films cast on a silicon wafer, an RGO film, single layer graphene, and a MWCNT film. The insets are the height profiles along the red line in each image.

Figure 4.1 Diagram showing the processes that occur within a thin film on an electrode surface during electrocatalysis. The symbols are as follows: $k$ is the rate constant for the catalytic reaction; $\mathrm{DH}^{+}$is the diffusion coefficient of protons within the film; $\mathrm{DH}_{2}$ is the diffusion coefficient of hydrogen within the film; $\mathrm{D}_{\mathrm{e}}$ is the diffusion coefficient of electrons within the film; $\mathrm{Co}(\mathrm{III})$ is oxidized form of the catalyst; $\mathrm{Co}(\mathrm{II})$ is the reduced form of the catalyst after one electron transfer.

Figure 4.2 (Left) Representative AFM image of an edge site on a $200 \pm 100 \mathrm{~nm}$ film. The film thickness was measured in this region by taking a profile across the blue dashed line and averaging vertically between the two horizontal white dashed lines. This averaged profile is shown on the right 
Figure 4.3 Background subtracted linear sweep voltammetry of a series of film thicknesses with a catalyst density of $2 \times 10^{-4} \mathrm{~mol}$ catalyst per $\mathrm{cm}^{3}$ of RGO. The supporting electrolyte was a deaerated $\sim 0.2 \mathrm{M}$ phosphate buffer at $\mathrm{pH} 2$. Scan rate was $10 \mathrm{mV} \mathrm{s}^{-1}$.

Figure 4.4 Plotted current and TOF versus film thickness from background-subtracted voltammetry in Figure 4.3. (a) Current at -0.95 V. (b) TOF at -0.95 V. (c) Current at -0.67 V. (d) TOF at $-0.67 \mathrm{~V}$. The data point corresponding to a $600 \mathrm{~nm}$ film does not follow a clear trend with the other data points and should probably be re-measured.

Figure 4.5 Background subtracted linear sweep voltammetry of a series of film thicknesses with $1.7 \pm 0.2 \times 10^{-10}$ mole catalyst in each film. The supporting electrolyte was a deaerated $\sim 0.2 \mathrm{M}$ phosphate buffer at $\mathrm{pH}$ 2. Scan rate was $10 \mathrm{mV} \mathrm{s}^{-1}$.

Figure 4.6 Plotted current (a) and TOF (b) versus film thickness from the background-subtracted voltammetry in Figure 4.5. 71

Figure 4.7 Background subtracted linear sweep voltammetry of three catalyst containing films. In blue is the voltammogram from Figure 4.5 (the $600 \pm 300 \mathrm{~nm}$ film containing $1.7 \pm 0.2 \times 10^{-10}$ mol catalyst). In black and red are voltammograms from similar films but with an additional 600 $\pm 300 \mathrm{~nm}$ RGO film cast on top of and below, respectively, the catalyst-containing film. Supporting electrolyte was a deaerated $\sim 0.2 \mathrm{M}$ phosphate buffer at $\mathrm{pH} 2$. Scan rate was $10 \mathrm{mV} \mathrm{s}^{-}$ 1 .

Figure 4.8 Illustration of a current versus thickness trend for an electrocatalytic film that follows the assumptions in the reports by Savéant and Costentin. ${ }^{222-224}$ The green circles are the current at a defined potential from cyclic voltammetry measurements. The dashed lines show the intersection between the linear portion at the beginning of the curve and the plateau region. The thickness at this intersection is the optimum film thickness.

Figure 4.9 Replotted data from Figure 4.4c with gray dashed lines corresponding to the linear portion at the lower end of the curve and the plateau current. The intersection of these dashed lines is at the optimum thickness $\boldsymbol{d}$ fopt.

Figure 4.10 Left, cyclic voltammogram of surface adsorbed 1 on an RGO thin film on a glassy carbon electrode in deaerated $0.1 \mathrm{M} \mathrm{KPF}_{6}$ and $2 \mathrm{mM}$ trifluoroacetic acid at a scan rate of $50 \mathrm{mV}$ $\mathrm{s}^{-1}$. Right, FOWA fitting using the data from the voltammogram on the left. The black dotted line is the data and the red line is the linear fit. The value for $E_{\text {cat } / 2}$ was taken as $-0.82 \mathrm{~V}$ (the potential at half the current of the peak current). The slope of the line is $6.6 \times 10^{-5} \mathrm{~A}$, which is equal to

2. 24RTFv122kCA012 76

Figure 5.1 Depictions of $2^{4+}, 3^{4+}, \mathrm{CB}[8]$, and $\mathrm{CB}[7]$. 81

Figure 5.2 Syntheses of $2^{4+}$ and $3^{4+}$. Details for these syntheses are described below. 83

Figure $5.3{ }^{1} \mathrm{H}$ NMR spectra of a) an aqueous $\mathrm{D}_{2} \mathrm{O}$ solution of $2^{4+}$ and b) an aqueous $\mathrm{D}_{2} \mathrm{O}$ solution of $3^{4+}$. The peaks are labeled corresponding to the protons in the structures above. 84 
Figure $5.4{ }^{13} \mathrm{CNMR}$ spectra of $2^{4+}$ (left) and $3^{4+}$ (right). The peaks are labeled corresponding to the carbon atoms in the structures above.

Figure 5.5 Equilibrium geometries modeled for free reduced dimers $2^{4+}$ and $3^{4+}$. Note the clear difference in the number of bond rotations needed to access the $\pi$-stacked geometries from these conformations: $2^{4+}<3^{4+}$

Figure 5.6 Equilibrium geometries modeled for free reduced dimers $2^{2+}$ and $3^{2+}$. Side view shows similar spacing of viologen groups, while end and top view illustrate the better $\pi-\pi$ stacking preorganization of $2^{2+}$.

Figure 5.7 ITC determination of binding constant between $2^{4+}$ and CB [8] (left) and $3^{4+}$ (right). All solutions for titration were made in phosphate buffer $(10 \mathrm{mmol})$ and degassed in vacuum. Solutions of $2^{4+}(0.3 \mathrm{mM})$ or $3^{4+}(0.4 \mathrm{mM})$ were titrated into a solution of CB $[8](0.026 \mathrm{mM})$, and the resulting exotherm curve was fit to obtain binding constants of $1.2 \times 10^{6} \mathrm{M}^{-1}$ for $2^{4+}$ with

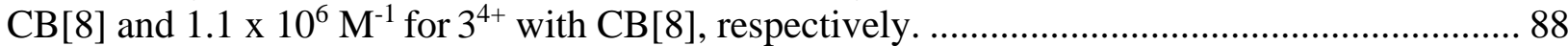

Figure 5.8 Cyclic voltammetry of viologen/cucurbituril pairs. From top to bottom: MV; 2; 3; MV + CB [7]; 2 + CB[7]; 3 + CB[7]; MV + CB [8]; 2 + CB [8]; 3 + CB [8]. Concentrations of MV, 2, and 3 , were $0.4,0.2$, and $0.2 \mathrm{mM}$, respectively. Concentrations of $\mathrm{CB}[7]$ and $\mathrm{CB}[8]$ were equivalent to the respective viologen in each panel. All voltammetry was performed in dearated $0.075 \mathrm{M}$ phosphate buffer at $\mathrm{pH}=7$ at a glassy carbon electrode and a scan rate of $0.05 \mathrm{~V} \mathrm{~s}^{-1} . .90$

Figure 5.9 Voltammetry of $2+\mathrm{CB}[8]$ (left) and $3+\mathrm{CB}[8]$ (right) at various scan rates collected at $0.25 \mathrm{mM}$ viologen with approximately one equivalent of $\mathrm{CB}$ [8]. The electrolyte was deaerated $0.075 \mathrm{M}$ phosphate buffer ( $\mathrm{pH} 7$ ). A glassy carbon electrode was used as the working electrode. Pt mesh was used as the counter electrode and a $\mathrm{Ag}$ wire coated with $\mathrm{AgCl}$ immersed in a fritted compartment containing saturated $\mathrm{KCl}$ was used as the reference electrode. 92

Figure $5.10{ }^{1} \mathrm{H}$ NMR spectra of a) aqueous $\mathrm{D}_{2} \mathrm{O}$ solutions of 2 in various oxidation states and in the presence of cucurbiturils and b) aqueous $\mathrm{D}_{2} \mathrm{O}$ solutions of 3 in various oxidation states and in the presence of cucurbiturils. Peaks labeled $j, k$, and $l$ correspond to cucurbituril protons. 93

Figure 5.11 Color variation in solutions of 2 with and without $\mathrm{CB}[8]$ after addition of reducing equivalents, defined as one reducing equivalent would reduce each viologen unity by $1 \mathrm{e}^{-}$. Top row: $2^{4+}$ (left); 2 after reduction with approximately half of the electrons necessary to fully reduce the solution to $2^{2+}$ (middle); 2 after the solution has been fully reduced to $2^{2+}$ (right). Bottom row: $2^{4+}$ with $\mathrm{CB}[8]($ left); 2 with $\mathrm{CB}[8]$ after reduction with approximately half of the electrons necessary to fully reduce the solution to $2^{2+}$ with $\mathrm{CB}[8]$ (middle); 2 with $\mathrm{CB}[8]$ after the solution has been fully reduced to $2^{2+} / \mathrm{CB}[8]($ right $)$. The solutions were $0.1 \mathrm{mM} 2$ in water. $\mathrm{CB}[8]$ was added in approximately 1 equivalent.

Figure 5.12 Absorbance spectra of 2 and 3 with and without cucurbiturils in solution after reduction to the $2+$ or $3+$ states. (left) $2^{2+}$ (black), $2^{2+}$ in the presence of an equivalent of CB[7] (blue), $2^{2+}$ in the presence of an equivalent of $\mathrm{CB}[8]$ (green), and $2^{3+}$ in the presence of an equivalent of $\mathrm{CB}[8]$ (red). (right) $3^{2+}$ (black), $3^{2+}$ in the presence of an equivalent of $\mathrm{CB}$ [7] (blue), $3^{2+}$ in the presence of an equivalent of $\mathrm{CB}[8]$ (green), and $3^{3+}$ in the presence of an equivalent of $\mathrm{CB}[8]$ (red)... 
Figure 5.13 a) Time-dependent absorbance spectra for $2^{2+}$ in aqueous solution recorded at $15 \mathrm{~s}$ intervals over $25 \mathrm{~min}$. b) Time-dependent absorbance spectra for $2^{2+}$ in same solution but with one equivalent of CB[8]. c) Time-dependence of the absorbance maxima in Figure 5.13a at $\lambda=$ $536 \mathrm{~nm}$ and in Figure 5.13b at $\lambda=623 \mathrm{~nm}$. d) Time-dependent absorbance spectra for $3^{2+}$ in aqueous solution. e) Time-dependent absorbance spectra for $3^{2+}$ in the same solution but with one equivalent of CB[8]. f) Time-dependence of the absorbance maxima in Figure 5.13d at $\lambda=$ $534 \mathrm{~nm}$ and in Figure 5.13e at $\lambda=622 \mathrm{~nm}$.

Figure 5.14 a.) Time-dependent absorption spectra for $\mathrm{MV}^{\cdot+}$ in phosphate buffer $(\mathrm{pH} 7)$ recorded at $15 \mathrm{~s}$ intervals over $25 \mathrm{~min}$. b) Time-dependent absorption spectra for $\mathrm{MV}^{{ }^{++}}$in same solution but with one equivalent of $\mathrm{CB}$ [8]. c) Time-dependence of the absorption maxima in Figure 5.14a at $\lambda=603 \mathrm{~nm}$ and in Figure $5.14 \mathrm{~b}$ at $\lambda=538 \mathrm{~nm}$.

Figure 5.15 Proposed elementary chemical and electrochemical steps involved in the reduction of 2 in the presence of $\mathrm{CB}[8]$.

Figure 5.16 a) The relative energies of molecular orbitals from DFT calculations (Spartan $16, \mathrm{~B} 97 \mathrm{D}, 6-311+\mathrm{G}^{*}$ ) showing the $3+$ and $2+$ states of 2 both without and within $\mathrm{CB}[8] . \mathrm{b}$ ) Graphical depiction of the LUMO of 2 in the $3+$ oxidation state and the sHOMO of 2 in the $2+$ oxidation state. c) The spin densities of 2 inside $\mathrm{CB}[8]$ in the $3+$ and $2+$ oxidation states are shown, with $\mathrm{CB}[8]$ omitted for clarity. Side and top views of the 2 are shown, with the blue regions highlighting the regions where the radical density is highest.

Figure 5.17 Energy minimized structure (hydrogens not shown) (a) and spin density map (b) of the $2\left(\mathrm{MV}^{+}\right)$pimer showing the known twisted conformation of the viologen moieties. 102

Figure 5.18 Structures of (left) cyclobis(paraquat- $p$-phenylene) and (right) cyclobis(paraquat- $o$ phenylene).

Figure 6.1 $\mathrm{Br} 3 \mathrm{~d}$ XP spectra of films casted from a GO suspension removed from a reaction mixture with 4-bromoaniline after the listed times.

Figure 6.2 Br 3d XP spectra (left) and C 1s XP spectra (right) of films casted on FTO from a suspension of $\mathrm{GO}$ after reaction with 4-bromoaniline. Each film was cycled in a $1 \mathrm{M} \mathrm{KCl}$ solution containing $1 \mathrm{mM} \mathrm{HCl}$ between open circuit potential and the potentials listed to the right in the figure three times. 108

Figure 6.3 Si 2p (left) and C 1s (right) XP spectra of GO films after reaction with TMPS. The top spectra are from a slurry of GO stirred in a solution of TMPs, and the bottom spectra are from a thin film of GO immersed in a solution of TMPS. In red are representative $\mathrm{C} 1 \mathrm{~s}$ spectra of an unreacted GO film. 109

Figure 6.4 FTIR spectra of GO (black) and GO after reaction with sodium azide (red). The arrow points to the N-N-N stretching peak.

Figure 6.5 I 3d (left) and $\mathrm{Cu} 2 \mathrm{p}$ (right) spectra of a GO-N 3 film after stirring in a solution of 6iodo-1-hexyne with a copper wire wrapped around the stir bar (red) and after stirring in a solution of 6-iodo-1-hexyne with a normal stir bar. 
Figure 6.6 Photograph of the electrochemical cell used for XAS experiments. Front view (left) and top view (right). Kapton film was glued (using super glue) across the window in the view on the left. The electrodes were inserted through the holes on the top of the cell in the view on the right and the solution was purged via an $\mathrm{N}_{2}$ line inserted through the smallest round hole. The cell is not air-tight, but a blanket of $\mathrm{N}_{2}$ was kept flowing over the top of the solution during experiments.

Figure 6.7 Overlay of normalized Co K-edge XANES spectra for two different samples at neutral $\mathrm{pH}$ (no added acid). Sample 1 was taken after soaking the electrode in a solution of 1 for about 4 hours on day 1, and Sample 8 was taking after soaking the electrode for about 2 days on day 5. The electrolyte was deaerated $0.1 \mathrm{M} \mathrm{KPF}_{6}$

Figure 6.8 Overlay of Co K-edge XANES spectra with the electrode held at various potentials at each $\mathrm{pH}$. Each electrode was first held at OCP, then brought to $-0.4 \mathrm{~V}$, then $-0.8 \mathrm{~V}$, then $-0.9 \mathrm{~V}$, and then it was brought back through the potentials in reverse order, ending at OCP. Hence, OCP (1st) was the first spectrum and OCP (2nd) the last spectrum taken in each case. (Note that at $\mathrm{pH}$ 2 , no data was taken at $-0.4 \mathrm{~V}$, hence the missing spectra.) The electrolyte was deaerated $0.1 \mathrm{M}$ $\mathrm{KPF}_{6}$ acidified with sulfuric acid. Each spectrum was taken on the same spot on the same (i.e. the sample position was not moved between scans).

Figure 6.9 Overlays of Co K-edge XANES spectra for individual carbon paper electrodes with adsorbed 1 at the highest (OCP) and lowest $(-0.9$ or $-0.8 \mathrm{~V})$ potentials over the range of $\mathrm{pH}$ values. The electrolyte was deaerated $0.1 \mathrm{M} \mathrm{KPF}_{6}$ acidified with sulfuric acid.

Figure 6.10 Fitted EXAFS data of a carbon paper electrode with 1 adsorbed, held at $-0.8 \mathrm{~V}$ vs $\mathrm{Ag} \mid \mathrm{AgCl}$. The electrolyte was deaerated in $0.1 \mathrm{M} \mathrm{KPF}_{6}$ and acidified to $\mathrm{pH} 1$ with sulfuric acid. Approximately 20 scans were taken and averaged.

Figure 6.11 Photocurrent response of a p-GaP(100) photoelectrode in deaerated $0.1 \mathrm{M}$ tetrabutylammonium perchlorate in acetonitrile with $10 \mathrm{mM}$ trifluoroacetic acid with various concentrations of 1 . A platinum mesh counter electrode and platinum wire quasi-reference electrode were used. The scan rate was $50 \mathrm{mV} / \mathrm{s}$ and the light intensity was $100 \mathrm{~mW} / \mathrm{cm}^{2}$ white light.

Figure 6.12 Photocurrent response of a methylated p-GaP(111A) photoelectrode with an RGO film in deaerated $0.1 \mathrm{M}$ tetrabutylammonium perchlorate in acetonitrile with $10 \mathrm{mM}$ trifluoroacetic acid with various concentrations of 1 . A platinum mesh counter electrode and platinum wire quasi-reference electrode were used. The scan rate was $50 \mathrm{mV} / \mathrm{s}$ and the light intensity was $100 \mathrm{~mW} / \mathrm{cm}^{2}$ white light. The dashed lines are the current in the dark.

Figure 6.13 Photocurrent response of a phenylated p-GaP(111A) photoelectrode with an RGO film before (black) and after (red) soaking in a $5 \mathrm{mM}$ solution of 1 in acetonitrile in a glovebox for 30 minutes. The supporting electrolyte was $0.1 \mathrm{M} \mathrm{KPF}_{6}$ in water with $10 \mathrm{mM}$ trifluoroacetic acid. A platinum mesh counter electrode and a $\mathrm{Ag} \mid \mathrm{AgCl}$ reference electrode were used. The scan rate was $50 \mathrm{mV} / \mathrm{s}$ and the light intensity was $100 \mathrm{~mW} / \mathrm{cm}^{2}$ white light. 121

Figure 6.14 Photocurrent response of phenylated p-GaP(111A) photoelectrodes with an RGO film (black and blue) and with an RGO film embedded with 1 (red and pink). The supporting 
electrolyte was $0.1 \mathrm{M} \mathrm{KPF}_{6}$ in water with $10 \mathrm{mM}$ trifluoroacetic acid. A platinum mesh counter electrode and a $\mathrm{Ag} \mid \mathrm{AgCl}$ reference electrode were used. The scan rate was $50 \mathrm{mV} / \mathrm{s}$ and the light intensity was $100 \mathrm{~mW} / \mathrm{cm}^{2}$ white light. 121

Figure 6.15 Possible functionalization of a gallium phosphide surface that can present both a hydrophobic (left) and hydrophilic (right) surface.

Figure 6.16 (left) Electrolysis of a silicon photoanode with (red) and without (black) an RGO thin film under $0.5 \mathrm{~V}$ and $100 \mathrm{~mW} / \mathrm{cm}^{2}$ white light. (right) Photocurrent response of the Si/RGO photoanode after the electrolysis. The electrolyte was $0.5 \mathrm{M} \mathrm{K}{ }_{4} \mathrm{Fe}(\mathrm{CN})_{6}$ in $0.2 \mathrm{M}$ phosphate buffer ( $\mathrm{pH}$ 7). Platinum mesh was used as the counter electrode and $\mathrm{Ag} \mid \mathrm{AgCl}$ was used as the reference electrode.

Figure 6.17 (a) Photoresponse of a silicon (111) photoanode functionalized with a benzyl alcohol group with (red) and without (black) an RGO film. (b) Dark current of the same electrodes as in (a). (c) Electrolysis under illumination at $0.5 \mathrm{~V}$ using the same electrodes as in (a) and (b). (d) Photoresponse of the electrodes after conducting the electrolysis in (c). The electrolyte was 0.5 $\mathrm{M} \mathrm{K}_{4} \mathrm{Fe}(\mathrm{CN})_{6}$ in $0.2 \mathrm{M}$ phosphate buffer $(\mathrm{pH} 7)$ and illumination in all cases was $50 \mathrm{~mW} / \mathrm{cm}^{2}$ white light. Platinum mesh was used as the counter electrode and $\mathrm{Ag} \mid \mathrm{AgCl}$ was used as the reference electrode.

Figure 6.18 (a) Photoresponse of a silicon (111) photoanode functionalized with a benzyl alcohol group with (red) and without (black) an RGO film after drying under vacuum. (b) Photoresponse of the electrodes after conducting the electrolysis in (c). (c) Electrolysis under illumination and $0.5 \mathrm{~V}$ using the same electrodes as in (a) and (b). The electrolyte was $0.5 \mathrm{M} \mathrm{K}_{4} \mathrm{Fe}(\mathrm{CN})_{6}$ in $0.2 \mathrm{M}$ phosphate buffer $(\mathrm{pH} \mathrm{7})$ and illumination in all cases was $50 \mathrm{~mW} / \mathrm{cm}^{2}$ white light. Platinum mesh was used as the counter electrode and $\mathrm{Ag} \mid \mathrm{AgCl}$ was used as the reference electrode. 126

Figure 6.19 Si 2p XP spectra of the silicon photoanodes before and after electrolysis under illumination. (a) Silicon (111) functionalized with a benzyl alcohol group. (b) Silicon (111) functionalized with a benzyl alcohol group and with an RGO film that had been dried under vacuum.

Figure 6.20 Scheme for GO monolayer attachment on silicon oxide surfaces using APTES (left) and TEOS (right).

Figure 6.21 Atomic force micrographs of self-assembled GO films on silicon wafers. (Top) A silicon wafer that had been functionalized with APTES and then immersed in a methanolic suspension of $\mathrm{GO}$ at $70^{\circ} \mathrm{C}$ for two hours. (Bottom) Silicon wafers that had been functionalized with TEOS and then immersed in a methanolic suspension of GO at room temperature for 20 hours, 4 days, and 1 week. 


\begin{abstract}
Photoelectrochemical systems are a promising method for the conversion of solar energy to storable chemical fuels or charge carriers. Limitations on these systems include high overpotentials for driving redox reactions, which reduce the efficiency of energy conversion, and difficulties in long-term storage of charge carriers. The work described in this thesis addresses both issues. First, high overpotentials can be mitigated by the addition of an electrocatalyst. Heterogeneous molecular electrocatalysis is promising but reports in photoelectrochemical systems are limited. This thesis describes the use of reduced graphene oxide (RGO) thin films to immobilize molecular electrocatalysts on electrodes. Second, charge carriers used to store electrochemical energy are subject to poisoning by oxygen. This thesis presents the use of hostguest chemistry to stabilize a radical species in the presence of oxygen.

First, a novel method for the fabrication of RGO thin films is demonstrated. This method is the first report using dissolved outer-sphere reductants to reduce graphene oxide to RGO. As a result, these RGO films are reduced without heteroatom doping or over-reduction, common problems with previously reported inner-sphere reductions. Furthermore, this method is exceedingly gentle and can be performed with a variety of underlying substrates including soft organic, non-conducting, and chemically sensitive materials.

RGO thin films deposited on electrode surfaces are shown to adsorb the proton reduction electrocatalyst cobalt(III) bis(dichlorobenzenedithiolate), presumably through $\pi$-stacking interactions. The retention of the electrocatalyst under turnover conditions is poor on smooth films but excellent on rough films. It is therefore hypothesized that the $\pi$-stacking interactions are weak and may become disrupted during turnover, leading to fast loss of the catalyst from smooth surfaces. However, on rough RGO films, catalyst intercalation is possible, leading to mechanical trapping that prevents it from diffusing away during electrocatalysis. This work has implications for the field of small molecule surface modifications that employ $\pi-\pi$ interactions. Specifically, these interactions may be weak, but intercalation within a graphitic material can lead to enhanced retention of adsorbed species.
\end{abstract}


Taking advantage of these findings, I then devised a new approach where catalyst and GO are co-deposited, and I studied the kinetics of electrocatalytic proton reduction in the resulting RGO films that are embedded with the electrocatalyst. This is the first time that fundamental kinetics of an electrocatalytic RGO thin film have been investigated. It is shown that different processes are limiting depending on the thickness of the film. For films thinner than 200-500 nm, diffusion processes limit the current, whereas for thicker films, electrical conductivity of the film likely plays a role. These conclusions are relevant for maximizing current in electrochemical energy conversion systems, especially since RGO is commonly used as a catalyst support in such systems.

The second part of this thesis describes interactions of two bis-viologen species with the cage molecule cucurbit[8]uril (CB[8]). A unique viologen oxidation state is identified in the presence of $\mathrm{CB}[8]$, identifiable by its absorption spectrum. This species possesses extended stability in the presence of oxygen. Computations suggest that the presence of a buried SOMO is the origin of this enhanced stability. This work has relevance in energy storage systems such as redox flow batteries and solar redox batteries, where trace oxygen can poison solutions of reduced viologens. Extending the stability of viologens by entrapment within cage complexes is a promising method for improving the shelf-life of these species. 


\section{Chapter 1. Introduction}

\subsection{A societal need for clean energy}

For the last 1 million years or more, humans and our recent ancestors have been burning organic materials to produce energy. ${ }^{1}$ For most of that million years, we have used materials like wood, peat, and coal, but since the industrial revolution, humanity has become more dependent on fossil fuels like oil and gas. In every case, however, the burning of organic materials generates pollutants like ash and gaseous species such as carbon dioxide and $\mathrm{NO}_{\mathrm{x}}$. Since the global population has seen many periods of exponential growth over the last century, ${ }^{2}$ these emissions have recently resulted in the onset of an environmental crisis. Today energy usage is at an all-time high. In 2015, global energy consumption was 575 quadrillion Btu, and the US Energy Information Administration predicted energy consumption in 2040 to be more than $25 \%$ higher, around 739 quadrillion Btu. ${ }^{3}$ As a result, carbon emissions will increase proportionately. In 2011, the OECD reported a projected increase in $\mathrm{CO}_{2}$ emissions of $70 \%$ by $2050 .{ }^{4}$ And in turn, as a result of this increase in greenhouse gas emissions, global temperatures have increased by $1.8^{\circ} \mathrm{F}\left(1^{\circ} \mathrm{C}\right)$ since 1900 and are expected to rise $2.5^{\circ} \mathrm{F}\left(1.4^{\circ} \mathrm{C}\right)$ by $2050 .^{5}$ This warming will likely result in catastrophic changes to our world and climate, such as sea levels rising as much as 8 feet by 2100 and an increase in severe weather events. ${ }^{5}$ Furthermore, studies have shown that climate change disproportionately affects lower-income countries and communities, which also happen to be the groups of people least responsible for greenhouse gas emissions. ${ }^{6-8}$ Therefore, it is imperative that communities that are able to implement change do so whenever possible.

One important direction in which we are moving is the complete switch to renewable and emission-free energy sources. One of the most extensively discussed and well-known sources of clean, renewable energy is solar radiation, and for good reason: one hour of irradiance from the sun delivers more energy than the total energy consumed by humans in one year. ${ }^{9}$ This suggests that even with fractionally efficient solar cells covering a fraction of the earth's surface, we could potentially power the planet on solar energy alone. In reality, of course, there are other obstacles 
that limit the feasibility of a totally solar powered society. One major obstacle is the intermittency and non-uniformity of solar irradiance on the Earth's surface. For example, the sun does not shine at night, and in regions with high cloud cover there is much lower solar irradiance than in drier regions. In order to mitigate this disparity, research has been directed towards methods of converting solar energy into a form of energy that can be stored for use at times when the sun is not shining.

A promising method for solar energy storage is the conversion of sunlight to chemical fuels. The most common non-biological fuel generated by solar energy conversion is hydrogen gas through the water splitting reaction:

$$
\begin{gathered}
4 \mathrm{H}^{+}+4 \mathrm{e}^{-} \rightarrow 2 \mathrm{H}_{2} \\
2 \mathrm{H}_{2} \mathrm{O} \rightarrow \mathrm{O}_{2}+4 \mathrm{H}^{+}+4 \mathrm{e}^{-}
\end{gathered}
$$

$$
2 \mathrm{H}_{2} \mathrm{O} \rightarrow 2 \mathrm{H}_{2}+\mathrm{O}_{2}
$$

This is an exceptionally non-polluting reaction as the only reactant is water and the only two products are hydrogen and oxygen gasses.

The conversion of sunlight to fuel through an electrochemical reaction like the water splitting reaction can be conducted in a number of ways. The three most common methods are described here. First, sunlight can be converted directly to a chemical fuel through a photochemical process like photosynthesis. In this process, light absorbers, usually semiconductor nanoparticles ${ }^{10}$ or, less often, molecular absorbers, ${ }^{11}$ capture solar energy and drive a chemical transformation. This process is shown for a nanoparticle light absorber in Figure 1.1c. When the light absorber absorbs photons of the appropriate energy, it enters an excited state where the excited electron can be transferred to an electron acceptor in solution (for the water-splitting reaction this is $\mathrm{H}^{+}$ions). In the case of the nanoparticle in Figure 1.1c, a positively charged hole $\left(\mathrm{h}^{+}\right)$is also formed, which transfers to an electron donor in solution (in this case $\mathrm{H}_{2} \mathrm{O}$ ). The equivalent of $\mathrm{h}^{+}$in an excited molecular light absorber is the newly emptied SOMO that can accept an electron. In this way, a single light absorber drives both the proton reduction half-reaction $\left(2 \mathrm{H}^{+} \rightarrow \mathrm{H}_{2}\right)$ and the water oxidation half-reaction $\left(2 \mathrm{H}_{2} \mathrm{O} \rightarrow \mathrm{O}_{2}\right)$. In some cases, a co-catalyst is also added to improve the kinetics of the reaction. These are often solid state clusters (such as $\mathrm{MO}_{\mathrm{x}}$ or $\mathrm{MS}_{\mathrm{x}}$ materials) on the surface of the light absorbing nanoparticle or solution state coordination complexes. ${ }^{12}$ 
Alternatively, the formation of chemical fuels can be acheived using an electrochemical cell. In Figure 1.1a, a water splitting reaction is driven in an electrochemical cell by an external photovoltaic (PV). This system can be termed a PV-electrolyzer. In this setup, solar energy is capture by the PV device. A p-type and an n-type semiconductor are sandwiched together, creating a p-n junction. When these semiconductors absorb sunlight, electrons are excited into the conduction bands, and a potential difference is generated across the junction driving electron flow through an external circuit, which in this case is an electrochemical cell. The physics of photovoltaic operation is described in detail in Reference 13. Fuel generation is then accomplished in the electrolyzer by using the current supplied by the PV to drive the proton reduction and water oxidation half-reactions at a cathode and an anode, respectively, immersed in an electrolyte solution (Figure 1.1a). Several examples of PV-electrolyzer devices are reported in the references cited here. ${ }^{14-17}$

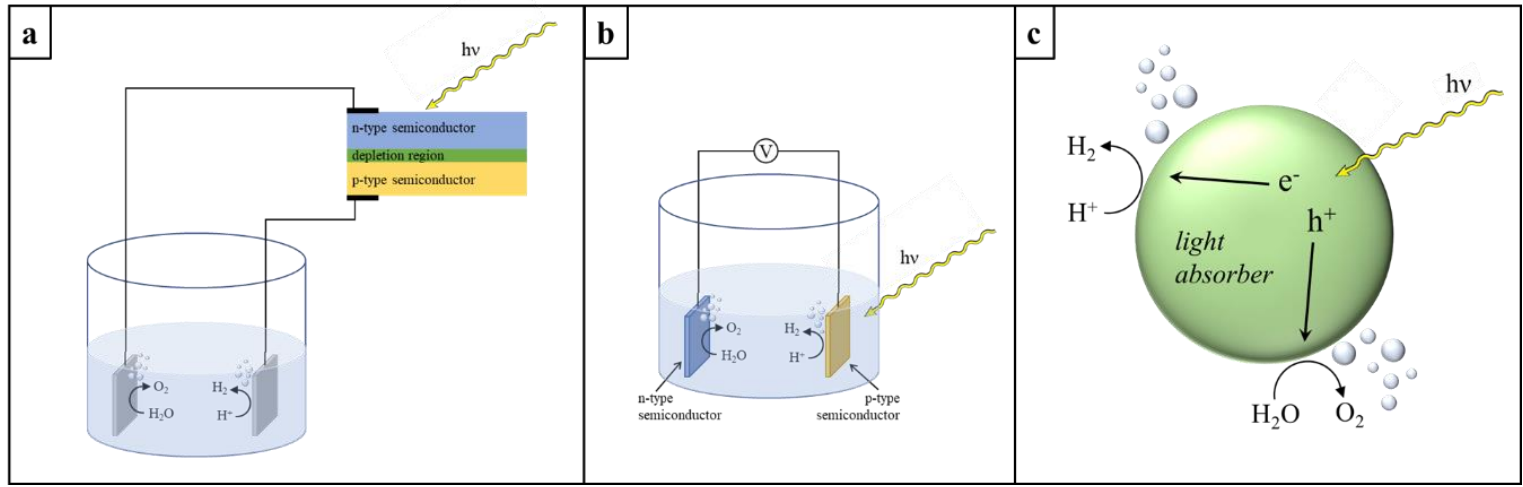

Figure 1.1 Three methods for solar water splitting: (a) electrolysis of water driven by an external photovoltaic, (b) photoelectrochemical water splitting, (c) photochemical water splitting.

Lastly, photoelectrochemical (PEC) cells can be understood as a hybrid of a PVelectrolyzer and a photochemical system. In this type of system (shown in Figure 1.1b), sunlight is converted to electricity (as in a regenerative PEC), a chemical fuel (e.g. a water splitting PEC), or both. ${ }^{18-20}$ The example shown in Figure $1.1 \mathrm{~b}$ represents a water splitting PEC. In a PEC, the electrodes themselves are semiconductors that absorb sunlight and drive a current in an external circuit, as in a PV device. The electron flow from this current then drives the redox reaction in solution, as in an electrolyzer. The cathode of a water-splitting PEC is a p-type semiconductor that can drive proton reduction; the anode is an n-type semiconductor that can drive water oxidation. ${ }^{21-}$ 
22 The major difference between a PV-electrolyzer and a PEC cell lies in the use of semiconductor materials both as electrodes and as light absorbers in the case of a PEC, whereas in a PVelectrolyzer, the light absorbing component is separate from the water splitting component.

\section{Beyond direct water splitting}

While the water splitting reaction is a popular source of chemical fuel because of the global abundance of water and the low environmental impact of burning $\mathrm{H}_{2}$, difficulties arise in the ability to store $\mathrm{H}_{2}$ long-term and to transport it. $\mathrm{H}_{2}$ is gaseous at ambient conditions so in order to store it with a high energy density, it must be compressed, which requires an energy input and introduces safety risks. Because of its low molecular weight, hydrogen molecules easily diffuse through containment walls leading to significant loss of $\mathrm{H}_{2}$ when stored for long periods of time. Additionally, $\mathrm{H}_{2}$ is combustible and can form explosive mixtures with $\mathrm{O}_{2}$, which is of particular risk when dealing with a leaky container of hydrogen. ${ }^{23}$

Non-gaseous fuels are easier and safer to store. These can include dissolved, liquid, and solid-state chemical fuels. For example, in redox flow batteries, molecules like organic radical polymers, vanadium oxide species, and organometallic complexes are used to store electrochemical energy. ${ }^{24-25}$ Alternatively, these redox species can be used as mediators in the water splitting reaction. In other words, the solar conversion methods described above (i.e. PEC, photochemistry, or PV) drive conversion of a redox species that can be stored as an energy source. When it is necessary to release the stored energy, these redox mediators can be combined with a proton reduction catalyst to facilitate spontaneous $\mathrm{H}_{2}$ generation by transfer of electrons from the redox species to $\mathrm{H}^{+}$ions. This is most often done using platinum nanoparticles as the catalyst. ${ }^{26-28}$ An example of this process is shown in Figure 1.2. In Figure 1.2a, species A is photoelectrochemically converted to $\mathrm{A}^{-}$(here $\mathrm{B}$ is a sacrificial reductant necessary to complete the electron transfer). Solutions of $\mathrm{A}^{-}$can be stored and transported without the safety risks associated with storing and transporting hydrogen gas; in this way, $\mathrm{A}^{-}$is a more practical energy storage material. In Figure 1.2b, $\mathrm{A}^{-}$is converted to a combustible fuel $\left(\mathrm{H}_{2}\right)$ by addition of a proton source and a proton reduction catalyst. In this scheme, the reduction potential of $\mathrm{A}^{-}$is sufficiently negative to drive reduction of protons, but the process is kinetically unfavorable unless a proton reduction catalyst is also present to facilitate the electron transfer. This means $\mathrm{A}^{-}$can be indefinitely stable in water solutions until platinum nanoparticles are added to the solution, in which case $\mathrm{H}_{2}$ is 
evolved. A further benefit of a system like this is that the energy conversion and storage aspects of PEC can be separately optimized.

a.) Electrochemical conversion and storage of solar energy

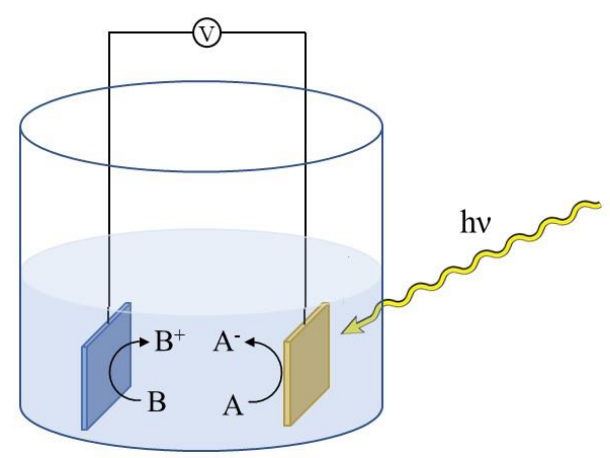

b.) Conversion of stored energy to a combustible fuel $\left(\mathrm{H}_{2}\right)$

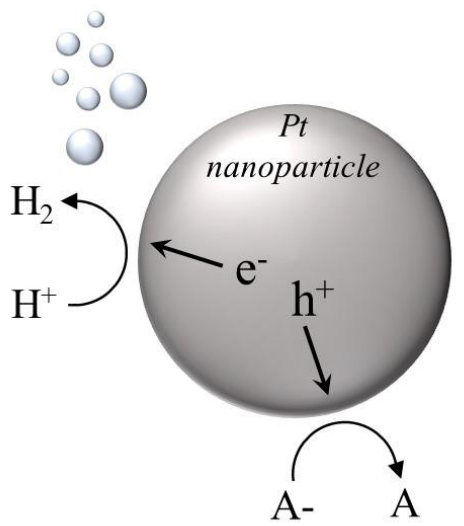

Figure 1.2 Example scheme of solar energy converted first (a) through the redox reaction $\mathrm{A} \rightarrow \mathrm{A}^{-}$ followed by (b) conversion to $\mathrm{H}_{2}$ by electron transfer through a proton reduction catalyst.

\subsection{Photoelectrochemical cells for solar energy conversion.}

Photoelectrochemical solar energy conversion has advantages over both photochemical and PV-electrolysis conversion of solar energy. First, there are two major advantages of a PEC over photochemical conversion. One of these is the ease of separation of the products. During the water splitting reaction, gaseous $\mathrm{H}_{2}$ and $\mathrm{O}_{2}$ are evolved simultaneously. In photochemistry these two species are created essentially at the same reaction site (in Figure 1.1c, both reactions happen simultaneously at the same nanoparticle); hence a subsequent separation step is required to obtain pure $\mathrm{H}_{2}$. In a $\mathrm{PEC}, \mathrm{H}_{2}$ and $\mathrm{O}_{2}$ are formed at physically separate electrodes. The separate collection of these two products is achieved readily by use of a membrane separator in the cell. A second advantage of PEC conversion over photochemical conversion lies in the ease of understanding and controlling fundamental aspects of the process. Advances in surface analysis methods make it easier to understand the chemistry of a macroscopic electrode compared to a discrete lightabsorbing particle. Furthermore, traditional electroanalytical techniques allow for an additional level of characterization of photoelectrode operation. 
The main advantage of a PEC over a PV-electrolyzer lies in its simpler design. Pristine semiconductors are expensive materials, as are the high surface area electrodes necessary to produce $\mathrm{H}_{2}$ efficiently. The cost of the conducting connective elements of a PV-electrolyzer device is also non-negligble. In a PEC, the semiconductors act simultaneously as the light absorbers and the electrodes, thereby simplifying the system and eliminating the costs of connective elements required in a PV-electrolyzer.

\section{Limitations on PEC efficiency}

Several limitations on water splitting PEC efficiency for solar energy conversion and storage currently exist. In the following, the limitations that are addressed by the research in this thesis are described.

First, large overpotentials can be detrimental in water-splitting electrochemical cell designs. Overpotential is any energy input beyond the thermodynamic energy of the reaction needed to drive the reaction. Three types of overpotential can occur in an electrochemical cell. ${ }^{29-}$ ${ }^{30}$ The first is activation overpotential, which generally arises from an activation energy barrier for the reaction. This is due to reaction kinetics and is specific to the electrode material and redox reaction. For example, proton reduction has an extremely low activation overpotential on platinum electrodes but a much higher activation overpotential on silver electrodes. ${ }^{31}$ The second is concentration overpotential, which arises from slow or limited diffusion of species to and from the electrode surface. This often observed when bubble formation blocks areas on the electrode surface, a common problem in the water splitting reaction. Lastly, resistance overpotential occurs because of cell design. This is common when junctions are formed in a cell, for example across a membrane separator. Specifically, an electrical resistance within the cell leads to a potential drop across that area.

This thesis focuses largely on mitigating the activation overpotential of the cathodic side of the water-splitting reaction. Activation overpotential of a particular reaction can be lessened by the addition of an electrocatalyst. Homogeneous (or solution phase) electrocatalysts are usually molecular species, most often coordination complexes. ${ }^{32}$ Many molecular catalysts mimic proton reduction and water oxidation catalysts in nature (e.g. hydrogenase). ${ }^{11,33}$ Heterogeneous catalysts can either be the same molecular catalysts immobilized on the electrode surface ${ }^{34-35}$ or solid state 
materials, such as metal nanoparticles, ${ }^{36}$ binary material films like $\mathrm{MoS}_{\mathrm{x}},{ }^{37}$ or metal-organic frameworks. ${ }^{38}$

A second limitation of PEC devices is the stability of photoelectrodes in solution and under bias. Many semiconductor materials that have been investigated for water splitting exhibit low stability in water and under illumination and bias. For example, n-type silicon, a common photoanode material, is prone to oxidative attack by the solvent under positive applied bias in water. Many p-type III-V and II-VI semiconductors (i.e. those comprised of elements from the third and fifth rows or the second and sixth rows of the periodic table) are also prone to selfreduction under negative bias. For example, the $\mathrm{Ga}$ atoms in $\mathrm{GaP}$ can reduce to metallic $\mathrm{Ga}^{0}$ clusters under a mild negative bias, resulting in a disruption of the crystal lattice and allowing for chemical attack. To prevent oxidative and reductive poisoning of semiconductor photoelectrodes, research in surface passivation techniques has been pursued. These techniques can be divided into two categories: chemical passivation and physical passivation (with some systems operating by both methods). In the former, dangling bonds at the surface are saturated by covalent grafting of a functional group to the surface. The latter operates by deposition of an organic or inorganic layer on the semiconductor surface that physically blocks reactive species (like water or $\mathrm{O}_{2}$ ). Covalent grafting is surface specific. For example, Grignard type reactions work well on silicon surfaces ${ }^{39}$ whereas indium phosphide can be passivated by thiol self-assembled monolayers. ${ }^{40}$ Physical passivation is generally surface independent and a variety of materials have been investigated for this purpose, including metal oxides ${ }^{41}$, organic polymers ${ }^{42}$, and graphene ${ }^{43}$.

\subsection{Heterogeneous molecular electrocatalysis.}

The setup of a water-splitting electrolysis cell is shown in Figure 1.3. The anode and cathode are immersed in electrolyte solutions and separated by a membrane that allows for ion diffusion between the two compartments but limits transport of dissolved $\mathrm{H}_{2}$ and $\mathrm{O}_{2}$. The two gaseous products can then be collected separately as they are evolved into the headspace of their respective half-cells.

This thesis discusses electrocatalysis in electrochemical and photoelectrochemical cells, in particular, the use of molecular heterogeneous electrocatalysts. In heterogeneous electrocatalysis, the catalyst is immobilized on the electrode surface. In contrast, in homogeneous electrocatalysis, the catalyst is dissolved in the electrolyte solution (the electrolyte reservoir in Figure 1.3). 
Heterogeneous electrocatalysis has several benefits over its homogeneous counterpart. For one, it significantly limits the amount of catalyst required. At a given moment in time, only the catalyst at the electrode surface is active. In other words, only catalyst at the electrode surface can undergo electron transfer with the electrode and participate in electrocatalysis. This means that catalyst dissolved in the electrolyte reservoir is largely inactive. Second, heterogeneous catalysis limits catalyst transport across ion exchange membranes in electrolysis cells, a source of contamination during long-term electrolysis. And lastly, heterogeneous electrocatalysis allows the scope of usable catalysts to expand beyond only water-soluble catalysts. This thesis specifically investigates heterogeneous molecular electrocatalysts. The advantage of molecular electrocatalysts over solid state electrocatalysts lies in well-defined active sites and a greater degree of tunability of molecular species.

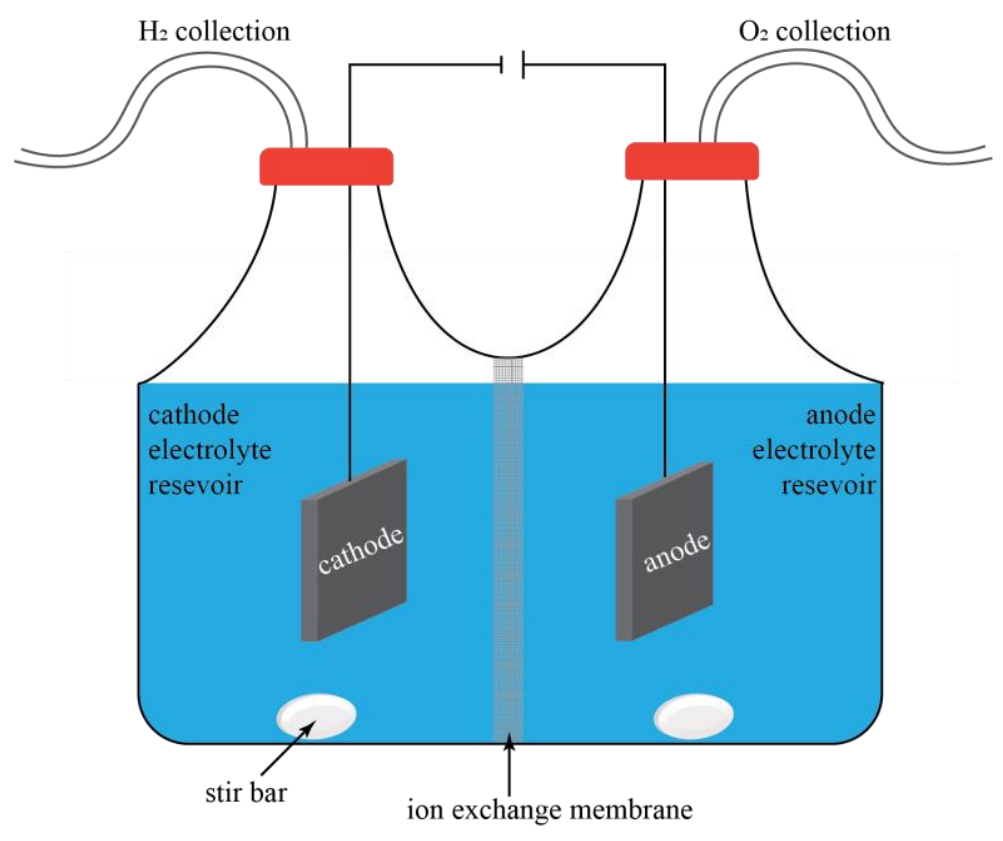

Figure 1.3 Example setup of a water-splitting electrolysis cell.

While much research has been devoted toward developing new bulk heterogeneous ${ }^{37-38,44-}$ ${ }^{45}$ and homogeneous molecular water splitting electrocatalysts, ${ }^{46-48}$ heterogenization of molecular electrocatalysts is less well-developed. ${ }^{47,49-51}$ Various strategies for immobilizing electrocatalysts on electrode surfaces have been explored, and Table 1.1 lists several examples of immobilized proton reduction electrocatalysts and their kinetic parameters. In this table, TOF and TON are the 
turnover frequency and turnover number, respectively, of each system. TOF is the average number of hydrogen molecules generated per catalyst site. It is usually calculated from an electrolysis experiment in which the electrode is held at a specific potential for a certain amount of time. The total charge passed during the electrolysis is then converted to moles of $\mathrm{H}_{2}$. If the Faradaic efficiency is $100 \%$ (i.e. percentage of electrons that are used to produce $\mathrm{H}_{2}$ ), for example, then all of the current goes towards $\mathrm{H}_{2}$ production, so moles of $\mathrm{H}_{2}$ produced $=1 / 2$ (moles of electrons passed during electrolysis). An equation for calculating TOF when Faradaic efficiency is $100 \%$ is shown in Equation 1.1, where $q$ is the charge passed in Coulombs, $F$ is the Faraday constant in Coulombs per mole, $t$ is the time of the electrolysis experiment, and $n$ is the number of moles of catalyst. If the Faradaic efficiency is less than $100 \%$, then the moles of $\mathrm{H}_{2}$ may be quantified using gas chromatography and can replace $\frac{q}{2 F}$ in equation 1.1.

$$
T O F=\frac{q}{2 F t n}
$$

It should be noted that TOF is highly dependent on applied potential and solution conditions (e.g. $\mathrm{pH})$ because it is related to current. This makes it difficult to directly compare different electrocatalytic systems.

TON is the total number of turnovers achieved during an electrolysis. This number is usually used as a measure of the stability of a system. For example, an electrocatalytic system may have a high turnover frequency for the first few minutes of electrolysis, but if the catalyst decomposes quickly, then the TON after an electrolysis experiment of several hours will be low. It should be noted that in Table 1.1, the time over which TON was calculated for each system varies greatly. TON is also, like TOF, dependent on applied potential and solution conditions, leading to further difficulties in comparing electrocatalytic systems.

Another difficult aspect in the comparison of electrocatalytic systems is the measure of overpotential. Overpotential is usually defined as the potential required to obtain a specific current density in an electrocatalytic system as compared to the potential required to obtain the same current density using a platinum electrode under the same conditions. However, because of differences in the choice of current density, catalyst surface loading, and actual electrode surface 
area (as opposed to geometric surface area), among other things, overpotential is difficult to compare among different systems.

In summary, TOF, TON, and overpotential are all parameters that can be used to compare the efficiency and stability of electocatalytic systems; however, because of differences in how these parameters are measured, calculated, and reported, it can be difficult to directly compare systems. Methods for obtaining these parameters should always be clearly reported and taken into account when comparing systems.

The electrocatalytic systems listed in Table 1.1 are all comprised of molecular electrocatalysts immobilized on inert electrode surfaces. The strategies for immobilization listed here can be divided into two subgroups: covalent attachment and noncovalent attachment, i.e. physical adsorption (termed "physisorption"). The former involves a chemical bond between the catalyst and the surface, and the latter involves the use of attractive van der Waals forces or entropic forces between the catalyst and the surface, for example charge-charge attractive forces or $\pi$-stacking interactions.

Covalently grafted catalysts are often more robust than physisorbed systems because of the strength of a chemical bond compared with a physical interaction. Covalent attachment, however, is inherently surface-specific. Certain electrode surfaces have received more attention than others for covalent attachment of molecular species. For example, metal oxides have been studied extensively for their ability to bind molecules containing phosphonate, carboxylate, and siloxane moieties. ${ }^{52-53}$ Silicon can easily be functionalized by Grignard reagents. ${ }^{39}$ And carbon-based electrodes can be functionalized with small molecules by targeting the variety of functional groups inherent to carbon surfaces, e.g. $\mathrm{C}=\mathrm{C}$ double bonds, carboxylates, and epoxides, among others. ${ }^{54-}$ ${ }^{55}$ Some work on covalent attachment to electrode surfaces is discussed in Chapter 6 of this thesis.

Physisorption, on the other hand, utilizes attractive van der Waals forces or entropic forces to immobilize small molecules on surfaces. These forces are most often charge-charge interactions $^{56-58}$ and $\pi-\pi$ interactions. ${ }^{54-55}$ These attachment methods are often less robust compared to covalent attachment methods, resulting in loss of catalyst from the surface over time. Physisorption, however, often has the advantage of requiring fewer synthetic steps than covalent attachment. For example, the physisorbed systems described in Chapters 3 and 4 of this thesis require no synthesis for the surface attachment. 


\begin{tabular}{|c|c|c|c|c|c|c|c|c|}
\hline Catalyst & Electrode $^{a}$ & linkage type & $\begin{array}{c}\text { overpotential } \\
(m V)\end{array}$ & $\begin{array}{c}\text { surface } \\
\text { coverage (mol } \\
\left.\mathrm{cm}^{-2}\right) \\
\end{array}$ & $\operatorname{TOF}\left(s^{-1}\right)$ & TON (time) & $p H$ & Reference \\
\hline $\begin{array}{c}\text { Nickel } \\
\text { bis(diphosphine) }\end{array}$ & MWCNT & covalent & $300^{b}$ & $1.5 \times 10^{-9}$ & $5.6^{c}$ & $1 \times 10^{5}(10 \mathrm{hr})$ & - & 59 \\
\hline $\begin{array}{c}\text { Nickel } \\
\text { bis(diphosphine) }\end{array}$ & MWCNT & noncovalent & $\sim 0$ & $2 \times 10^{-9}$ & $3.9^{d}$ & $8.5 \times 10^{4}(6 \mathrm{hr})$ & 0.3 & 60 \\
\hline Rhodium complex & carbon black & noncovalent & 350 & $2.6 \times 10^{-9}$ & $0.95^{e}$ & $206(1.25 \mathrm{hr})$ & 1.9 & 61 \\
\hline 1D Cobalt dithiolene & glassy carbon & noncovalent & $\sim 500$ & $5 \times 10^{-7}$ & - & $f$ & 1.3 & 62 \\
\hline Cobaloxime & MWCNT & covalent & 350 & $4.5 \times 10^{-9}$ & $2.2^{g}$ & $5.5 \times 10^{4}(7 \mathrm{hr})$ & 4.5 & 63 \\
\hline Cobaloxime & $\begin{array}{l}\text { Mesoporous } \\
\text { ITO }\end{array}$ & covalent & 300 & $1.5 \times 10^{-7}$ & - & - & 7 & 64 \\
\hline Hydrogenase & HOPG & covalent & 800 & - & - & $h$ & 1 & 65 \\
\hline Cobalt clathrochelate & glassy carbon & covalent & 800 & - & - & - & 1 & 66 \\
\hline Cobalt corrole & CNT & covalent & $800^{j}$ & - & 99 & ${ }^{k}(10 \mathrm{hr})$ & 14 & 67 \\
\hline $\begin{array}{c}\mathrm{W}_{2} \mathrm{O}_{2}(\mu-\mathrm{S})_{2}\left(\mathrm{~S}_{2}\right) \\
\left.\left(\mathrm{S}_{4}\right)\right]^{2-}\end{array}$ & glassy carbon & noncovalent & $227^{l}$ & - & 93 & $m$ & 0 & 68 \\
\hline $\begin{array}{c}\text { Cobalt bis(dichloro- } \\
\text { benzenedithiolate) }\end{array}$ & HOPG & noncovalent & 590 & $2.6 \times 10^{-10}$ & $320^{i}$ & $9.1 \times 10^{6}(8 \mathrm{hr})$ & 0.3 & 69 \\
\hline
\end{tabular}

${ }^{a}$ MWCNT $=$ multi-walled carbon nanotubes; ITO $=$ indium tin oxide $; \mathrm{HOPG}=$ highly ordered pyrolytic graphite $; \mathrm{CNT}=$ carbon nanotubes. ${ }^{b}$ potential required to produce $4 \mathrm{~mA} \mathrm{~cm}^{-2} .{ }^{c}$ at $-0.5 \mathrm{~V} .{ }^{d}$ at $-0.3 \mathrm{~V} .{ }^{e}$ at $2 \mathrm{~mA} \mathrm{~cm}^{-2} .{ }^{f}$ low stability due to delamination. ${ }^{g}$ at $-0.59 \mathrm{~V} .{ }^{h}$ very low stability. ${ }^{i}$ averaged over 8 hr electrolysis. ${ }^{j}$ at a current of $12.1 \mathrm{~mA} \mathrm{\mu g}{ }^{-1} .{ }^{k}$ no change in current over $10 \mathrm{hrs} .{ }^{l}$ at a current of $10 \mathrm{~mA} \mathrm{~cm}{ }^{-2} .{ }^{m}$ stability tested by cycling, no change after $1000 \mathrm{cycles}$.

Table 1.1 Reported molecular proton reduction catalysts immobilized on inert electrodes. 


\begin{tabular}{|c|c|c|c|c|c|c|c|c|}
\hline Catalyst & semiconductor & linkage type & $\begin{array}{l}\text { onset potential } \\
(\mathrm{mV})\end{array}$ & $\begin{array}{c}\text { surface coverage } \\
\left(\mathrm{mol} \mathrm{cm}^{-2}\right)\end{array}$ & Stability & $\mathrm{pH}$ & $\begin{array}{l}\text { Illumination } \\
\left.(\mathrm{mW} \mathrm{cm})^{-2}\right)\end{array}$ & Reference \\
\hline $\begin{array}{c}\text { nickel } \\
\text { phosphine }\end{array}$ & Si (111) & covalent & $-60^{a}$ & $2.5 \times 10^{-10}$ & - & 1 & $33^{d}$ & 70 \\
\hline $\mathrm{Mo}_{3} \mathrm{~S}_{4}$ & Si pillars & noncovalent & $150^{b}$ & - & $\begin{array}{l}>60 \mathrm{~min} \\
\text { electrolysis }\end{array}$ & 0 & $28.3^{e}$ & 71 \\
\hline $\mathrm{Fe}_{2} \mathrm{~S}_{2}(\mathrm{CO})_{6}$ & InP nanocrystals & noncovalent & $>250^{b}$ & - & - & 7 & $f$ & 72 \\
\hline Cobaloxime & $\mathrm{GaP}$ & covalent & $240^{c}$ & $11 \times 10^{-9}$ & $\begin{array}{c}>60 \text { min } \\
\text { electrolysis }\end{array}$ & 7 & 100 & 73 \\
\hline $\begin{array}{l}\text { 1D Cobalt } \\
\text { dithiolene }\end{array}$ & $\mathrm{Si}$ & noncovalent & $\sim 200$ & $5 \times 10^{-7}$ & $\begin{array}{l}>20 \mathrm{~min} \\
\text { electrolysis }\end{array}$ & 1.3 & 1 sun & 62 \\
\hline
\end{tabular}

${ }^{a}$ vs NHE. ${ }^{b}$ vs SHE. ${ }^{c}$ vs RHE. ${ }^{d}$ LED white light. ${ }^{e}$ red light, $\lambda>620$ nm. ${ }^{f} 395 \mathrm{~nm}$ diode (no intensity given).

Table 1.2 Reported molecular proton reduction catalysts immobilized on semiconductor photocathodes under illumination. 
It is important to note that for both covalent and noncovalent immobilization methods listed in Table 1.1, carbon materials comprise the majority of electrode surfaces used. This is representative of literature reports of small molecule immobilization on electrode surfaces, suggesting that immobilization on carbon materials is more well-understood than immobilization on any other electrode material.

The complex in the last row of Table 1.1 (cobalt bis[dichlorobenzenedithiolate], complex 1 shown in Figure 1.4) is the catalyst that will be discussed in several chapters of this thesis in terms of immobilization on electrode surfaces. This catalyst was chosen for four reasons: (1) its previously demonstrated adsorption on graphitic carbon electrodes (Table 1.1), (2) its high catalytic activity when immobilized on an electrode (highest

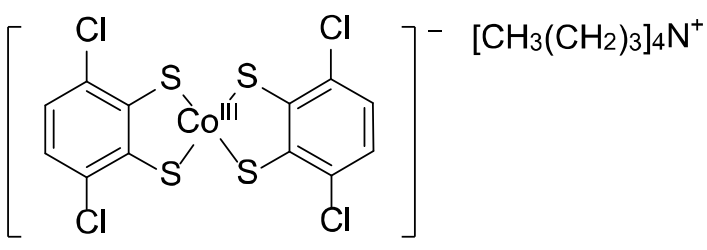

Figure 1.4 Structure of 1. TOF and TON of all systems listed in Table 1.1), (3) ease of synthesis, and (4) the potential to modify the ligands for covalent attachment to surfaces.

Further considerations are necessary when envisioning a heterogenized molecular electrocatalytic system for solar water splitting. Specifically, immobilization on light-absorbing semiconductor materials is necessary. As already mentioned, a vast majority of reported techniques for immobilizing molecular catalysts on electrode surfaces use carbon materials as the electrode (as evidenced by Table 1.1). Unfortunately, however, carbon materials are not lightabsorbing. Therefore, these techniques are not useful for immobilizing molecular catalysts directly on photoelectrode surfaces. Immobilization of molecular proton reduction electrocatalysts on light absorbing materials is comparatively less well researched than that on inert carbon materials. Table 1.2 lists a few examples of such systems. As with electrocatalysis, these photoelectrocatalytic systems are difficult to compare directly with each other. This is exacerbated in photoelectrocatalytic systems by the addition of variable parameters such as light intensity and wavelength, dopant density in the semiconductor, and stability of the semiconductor itself. These parameters will not be discussed at length here. An in-depth description of electron transfer using semiconductor electrodes can be found in the following references 74-75.

The surface chemistry of an electrode can be altered by addition of an ultrathin layer of some material. The surface chemistry of the electrode is then defined by this material rather than the electrode itself. Therefore, a material that can be deposited in an ultrathin layer on any electrode 
regardless of the identity of the electrode allows for universal immobilization chemistry on electrode surfaces. Because there is such a large pool of research that has already been devoted to immobilization methods on graphitic carbon, thin films of graphitic carbon on photoelectrode surfaces are highly desirable. The requirements for such a material are threefold: (1) high optical transparency to allow light absorption by the underlying semiconductor, (2) electrical conductivity to allow transfer of electrons between the semiconductor and the catalyst at the surface, and (3) a well-defined and consistent surface chemistry allowing for immobilization of small molecules. The development of graphene-like films on electrode surfaces for the immobilization of small molecules is a major focus of this thesis. An additional benefit of graphene modification of semiconductors surfaces lies in its ability to passivate the surface (i.e. protect against corrosion). ${ }^{43}$, 76-77

\subsection{Reduced graphene oxide.}

Graphene is a 2D material ( 1 atom thick), consisting of an extended aromatic network of carbon. Because of the aromaticity, it is electronically conductive, and because of its thinness it is highly optically transparent. Graphene is generally fabricated by CVD methods ${ }^{78}$ or by exfoliation from graphite, as in the "scotch tape method". 79 These methods, however, are not amenable to fabricating large area sheets of graphene. CVD is energy intensive and graphite exfoliation results in flakes of graphene up to only millimeters in diameter.

Reduced graphene oxide (RGO, sometimes called rGO) is a graphene-like material that will be discussed in depth here. RGO has three major advantages over pristine graphene: (1) it is easily and cheaply synthesized from graphite through solution methods; (2) films can be cast from a solution-processable precursor, allowing for facile, controllable, and low-cost deposition of thin films; and (3) RGO has a large variety of surface oxygen functional groups, allowing for a range of methods for grafting small molecules to the surface. The structures of graphene and RGO are shown in Figure 1.5. Graphene is a single, large-area sheet of aromatic carbon, whereas RGO is made up of smaller sheets of partially oxidized aromatic carbon. 


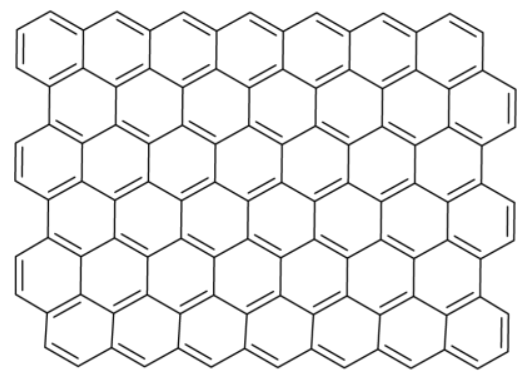

graphene

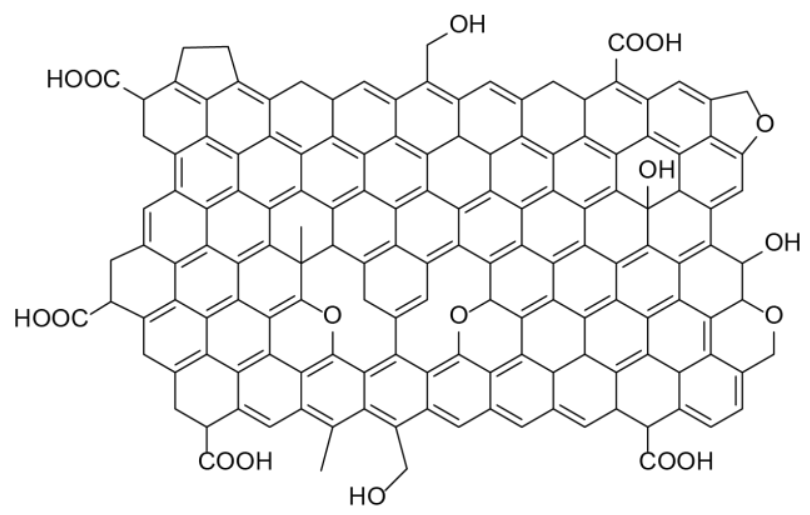

RGO

Figure 1.5 Structures of graphene (left) and RGO (right).

RGO is fabricated in four steps. This process is shown in Figure 1.6. The first step consists of oxidizing graphite flakes using strong oxidants, such as sulfuric acid combined with $\mathrm{KMnO}_{4}{ }^{80}$ This creates a material known as graphite oxide, which retains the honeycomb molecular structure of graphite but not the aromaticity. Graphite oxide, therefore, is not electrically conductive. However, because of the oxygen groups created during the oxidation process, it is a hydrophilic material and can be exfoliated and suspended in water and some other polar protic solvents. This exfoliated, oxidized material is termed graphene oxide (GO). Suspensions of GO can be cast as thin films on a variety of surfaces by methods including dropcasting, spincasting, spray pyrolysis, Langmuir-Blodgett, and others. ${ }^{81-84}$ Once these films are cast, they can be reduced to RGO and in doing so, the aromatic network, and thereby the electrical conductivity, is restored.

RGO is useful for surface modification both through covalent grafting and physisorption. The former is achievable because of the variety of oxygen functional groups that remain even after reduction of GO. These groups consist largely of epoxides and hydroxides with smaller amounts of carbonyls and esters. This variety of functional groups allows for many different routes of grafting small molecules to RGO materials. For example, there are several reports on grafting to RGO through amide linkages ${ }^{85-87}$ and amine attack at epoxide groups. ${ }^{88-90}$ Additionally, RGO is useful for physisorption of small molecules because it can participate in $\pi$-stacking interactions. Small molecules containing a $\pi$-system that can lay flat on the RGO surface will spontaneously adsorb to that surface. Some molecular electrocatalysts like porphyrin complexes inherently 
contain these flat $\pi$-systems, and some must be modified with something like a pyrene "foot" that can dangle off the catalyst and adsorb to the RGO surface. ${ }^{60,91-92}$

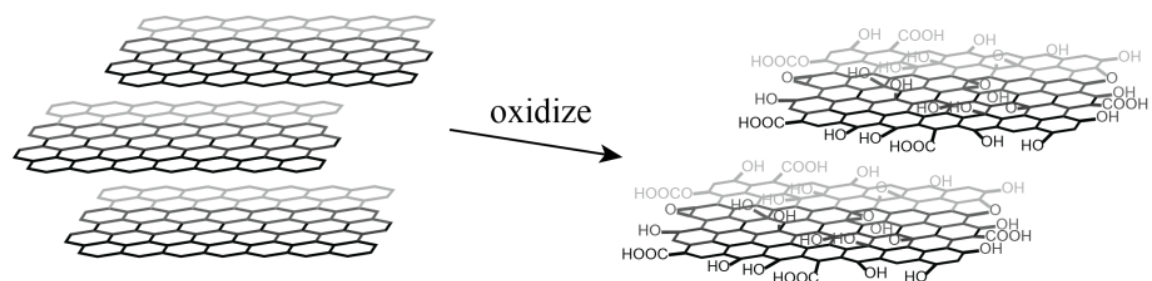

graphite

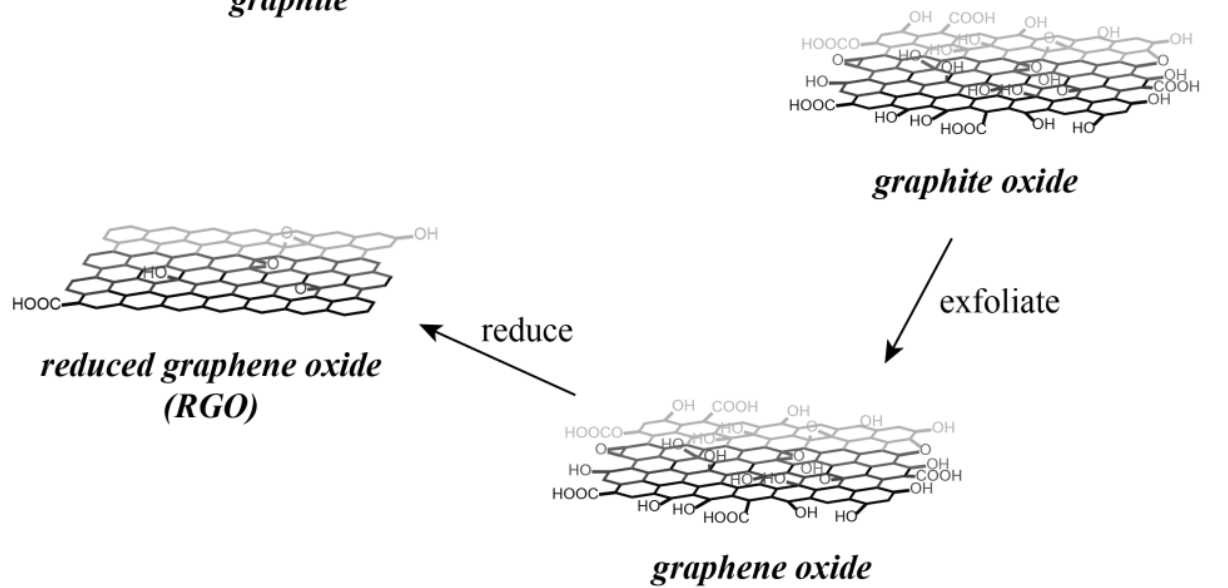

Figure 1.6 Scheme showing the synthetic steps to obtain RGO from graphite powder. Double bonds are omitted for the sake of clarity.

Several different oxidation methods have been used to produce GO from graphite powder, the most common being the named Hummers, Hofmann, and Staudenmaier methods. ${ }^{80,93-95}$ Each of these oxidation methods produce graphene oxide with slightly different chemical and structural properties. Even more varied, however, are the reduction methods published to date, which in turn produce chemically, structurally, and electronically varied RGO. ${ }^{96-98}$ For this reason, it is extremely important to have well-understood methods for the characterization of RGO. Chapter 2 of this thesis describes a new method for the fabrication of thin films of RGO and the characterization of that RGO. Here I discuss the methods commonly used to characterize RGO and other graphitic materials.

X-ray photoelectron spectroscopy (XPS) is an important surface characterization method used to determine the chemical composition of graphitic materials. The $\mathrm{C} 1 \mathrm{~s}$ and the $\mathrm{O} 1 \mathrm{~s}$ peaks can be deconvoluted to give information on the types and relative quantities of different carbon and oxygen functional groups within the material. Using the $\mathrm{O} 1 \mathrm{~s}$ peak to obtain this information, 


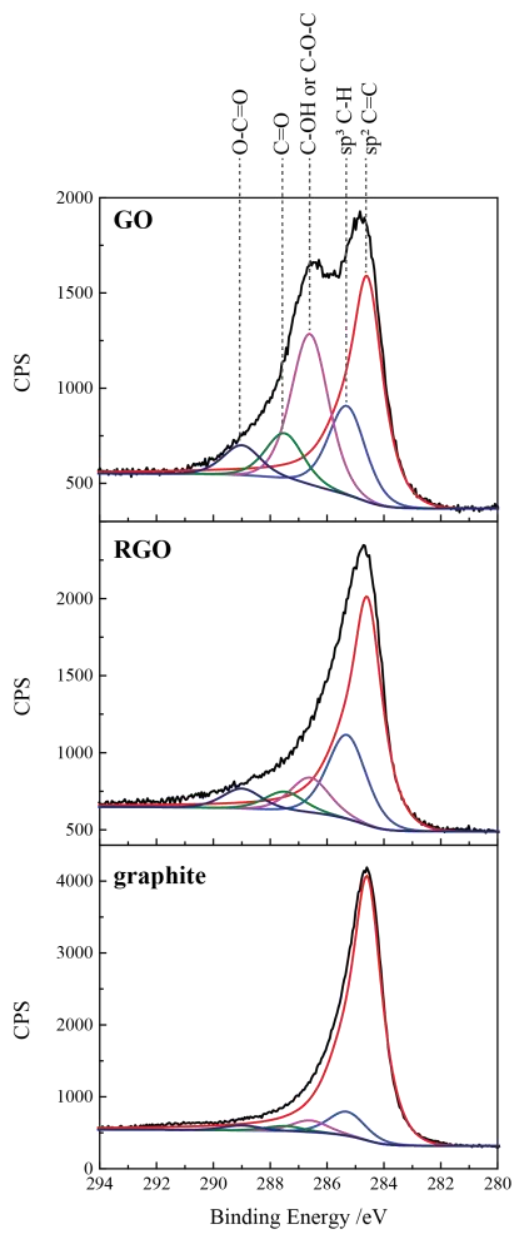

Figure 1.7 Sample set of XP C1s spectra of GO (top), RGO (middle), and graphite (bottom) that have been fitted with a series of Gaussian-Lorentzian peaks.

however, can be complicated by the presence of water within the material. Since RGO is fabricated through oxidation of graphite in an aqueous mixture and GO is hydrophilic and often suspended in water prior to reduction to RGO, residual water trapped in the RGO matrix can make up a large portion of the $\mathrm{O}$ 1s signal. The $\mathrm{C}$ 1s signal, however, is less ambiguous. A sample set of XP C1s spectra are shown in Figure 1.7. These spectra have been fitted with a series of peaks. The peaks correspond to the functional groups labeled in the figure. As can be seen, graphite is almost entirely $\mathrm{sp}^{2}$ carbon, as expected, while GO has large quantities of C-O functional groups. RGO contains an intermediate level of $\mathrm{C}-\mathrm{O}$ functionalities. The graphitic $\mathrm{C}=\mathrm{C}$ peak is fitted with an asymmetric function. The asymmetry of this peak is due low energy $\pi^{*} \rightarrow \pi$ transitions, known as shake-up satellites, that occur when an electron is ejected and a hole is left behind within the $\pi$-system of the graphitic structure. ${ }^{99-100}$ Each other type of carbon functionality can be fitted with a symmetric 
Gaussian-Lorentzian product. Because these functionalities are not part of the $\pi$-system their excitations do not produce shake-up satellites.

Importantly, fitted C1s XP not only give information on the total C-O content of graphitic materials but also the relative amounts of each type of $\mathrm{C}-\mathrm{O}$ functionality. For example, from the data shown in Figure 1.7, it is apparent that the reduction method used here (that is the CoCp$/ \mathrm{TFA}$ solution described in Chapter 2) greatly reduces the amount of $\mathrm{C}-\mathrm{OH}$ and $\mathrm{C}-\mathrm{O}-\mathrm{C}$ groups while having limited effect on the $\mathrm{O}-\mathrm{C}=\mathrm{O}$ (carboxylate) groups. Having this kind of information can help understand the mechanism of GO reduction, allowing for better control of that reduction. It can also help better understand the wetting of RGO in different solvents, the acidity of RGO, and the ability of RGO to undergo different types of covalent grafting procedures, among other things.

Raman spectroscopy is another useful tool for probing the structure of graphitic materials. Dresselhaus et al. give an in-depth description of the origin of Raman bands for single- and multilayer graphene and graphite. ${ }^{101-102}$ The four most distinctive Raman bands produced by graphitic carbon are termed the D, G, D', and G' (also called 2D or D*) bands and show up around 1300, 1580, 1620, and $2600 \mathrm{~cm}^{-1}$, respectively. In Figure 1.8, sample spectra of GO, RGO, and graphite are shown and the D, G, and G' peaks are labeled. In this case, the G and D' peaks overlap forming a single broad band identified simply as the $G$ peak. The pure $G$ band is so named because it is the only first-order Raman mode of graphitic carbon. It arises from the $E_{2 g}$ stretching of the graphitic matrix (i.e. $\mathrm{sp}^{2}$ carbon stretches). In graphene and highly ordered graphite, this is by far the most intense band. The D and D' bands are considered defect bands. They arise from $\mathrm{sp}^{3}$ carbon stretching. The $\mathrm{D}$ band has sometimes been explained as the symmetric $A_{l g}$ stretching mode, which becomes Raman-active as the symmetry of the graphitic network is broken. ${ }^{103}$ The G' band is an overtone of the $\mathrm{D}$ band (for this reason, some researchers call it the $2 \mathrm{D}$ or $\mathrm{D}^{*}$ band). It should be noted that both the $\mathrm{D}$ and $\mathrm{G}$ ' bands exhibit frequencies that are dependent on the laser excitation frequency because they arise from a double resonant process. Specifically, resonance selectively intensifies different modes from a set of modes depending on the wavelength of excitation. ${ }^{104}$

Because the $G$ peak arises from perfect graphitic carbon and the D peak arises from defective graphitic carbon, the relative intensities of these two peaks can give information on the level of defects within the graphitic material. Specifically, the $I_{D} / I_{G}$ ratio is inversely proportional to crystallite size and can be used to calculate the average crystallite size (that is, the average radius of defect-free graphitic regions). ${ }^{103,} 105$ However, this relationship is only valid when the 
crystallites are $>3 \mathrm{~nm}$. For materials with smaller crystallites, the relationship has a more complex dependence on crystallite size. ${ }^{106-107}$ Furthermore, because the G and D' bands are so close in frequency, they often overlap (as is the case in Figure 1.8) and present as a single broadened peak, usually still called the G peak. Therefore, the position and FWHM of this G peak can also give information on the relative defect density of graphitic materials. For example, the $\mathrm{G}$ peak in Figure 1.8 occurs at slightly different wavenumbers in GO $\left(\sim 1606 \mathrm{~cm}^{-1}\right)$ and RGO $\left(1597 \mathrm{~cm}^{-1}\right)$ and with a slightly narrower FWHM in RGO compared to GO.

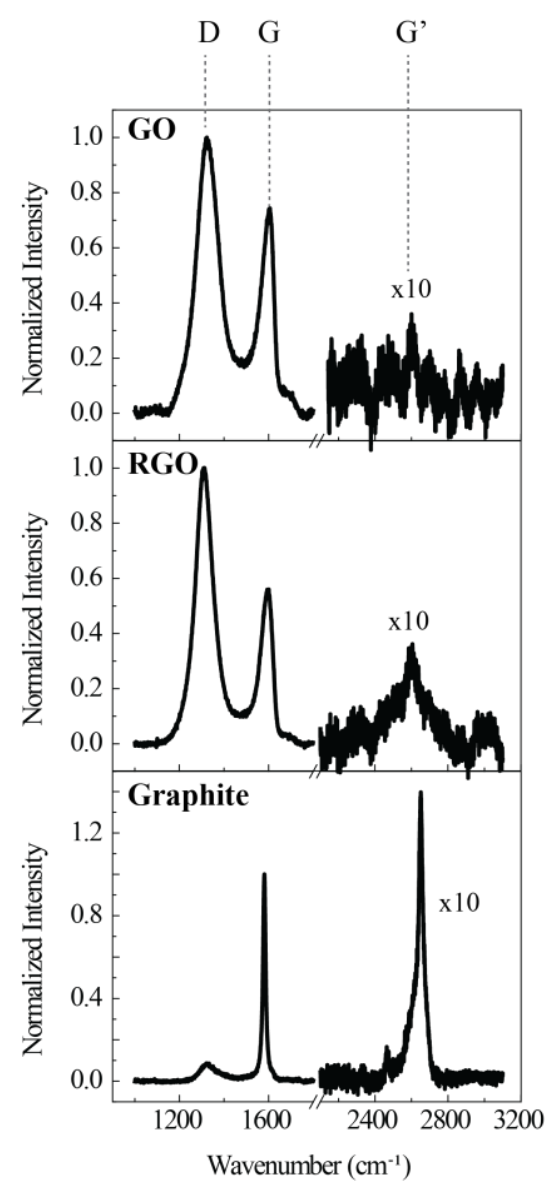

Figure 1.8 Sample Raman spectra of GO (top), RGO (middle), and graphite (bottom). The D, G, and G' bands are indicated. A $785 \mathrm{~nm}$ Raman laser excitation was used to collect these spectra.

Attempts to deconvolute the G, D, and G' peaks have been presented in the literature. Deconvolution of the $G$ peak is fairly straightforward as it is comprised mainly of the pure $G$ peak and the D' peak. These can be fitted with Lorentzian functions. ${ }^{108}$ Some reports have also attempted to deconvolute the D band using a set of Lorentzian functions; ${ }^{106,108-112}$ however, the physical origins of the peaks that comprise the $\mathrm{D}$ band are poorly understood, so these fittings vary 
considerably. Lastly, the G' band can give information on stacking defects in highly ordered materials such as few-layer graphene or HOPG (highly ordered pyrolytic graphite). Dresselhaus et al. have shown fittings of the G' peak for few-layer graphene and highly ordered graphite. ${ }^{101}$ In low order materials like graphite and RGO, the G' band appears as a broad peak, and in extremely low order materials, like GO, the G' band disappears altogether. ${ }^{113-114}$ The effect of G' peak broadening and disappearance can be seen in the spectra in Figure 1.8.

Other commonly used methods to characterize graphitic materials include atomic force microscopy (AFM) and electrical resistance measurements. The former is an imaging technique that generally has resolution down to $1 \mathrm{~nm}$, and in some cases atomic resolution ${ }^{115}$. Single RGO sheets can be imaged using this technique (Figure 1.9a) or for thicker RGO films, surface morphology, roughness, and thickness can be obtained (Figure 1.9b). Conductive AFM can be used to determine the sheet resistance of RGO, ${ }^{116-117}$ but when this technique is not available, fourpoint probe measurements are commonly used to measure sheet resistance. ${ }^{118-121}$
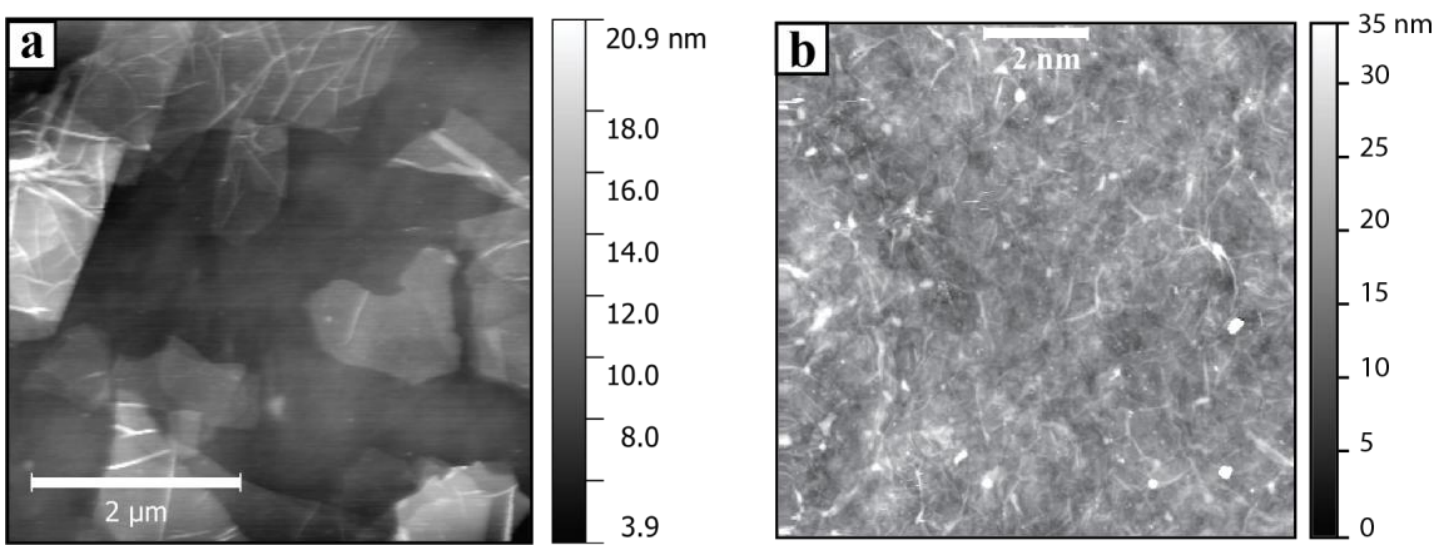

Figure 1.9 Sample AFM images of (a) individual sheets of GO and (b) a thicker film of RGO on silicon wafer surfaces.

Many other methods are available for the characterization of RGO materials. These methods include but are not limited to transmission and scanning transmission electron microscopy (TEM and STEM), ${ }^{122-126}$ scanning tunneling microscopy (STM), ${ }^{127-129}$ infrared absorbance (IR) spectroscopy, ${ }^{123}$, 130-132 X-ray diffraction (XRD), ${ }^{121-123,125-126,} 133$ thermogravimetric analysis (TGA), ${ }^{122,126,134-135}$ UV-visible absorbance spectroscopy, ${ }^{122,124-125,134}$ and NMR spectroscopy. ${ }^{120,}$ ${ }^{126}$ Because these methods were not used extensively in this thesis, they are not discussed in depth here; however, examples of their usage for the characterization of RGO and related materials can be found in the listed references. 


\subsection{Other redox species for electrochemical energy storage}

As mentioned previously in Section 1.1, a major hurdle in the implementation of hydrogen as a fuel is the difficulty of storage and transportation. Gaseous fuels have low energy density unless compressed, but compression requires an energy cost. Furthermore, $\mathrm{H}_{2}$ leakage from sealed containers is rapid compared with larger molecules, and hydrogen is explosive when mixed with oxygen. For these reasons, research into alternative redox species for energy storage is important. Organic redox species have the benefit of being relatively cheap and non-toxic with a high energy density compared to metal-based species. In particular, viologens (bipyridine derivatives) have received significant attention, especially in the redox flow battery community. These compounds have extremely fast charge transfer rate constants, high reversibility, and long charge-discharge cycling lifetimes. Furthermore, they are highly water soluble and easy to derivatize. A disadvantage of viologens is the reactivity of the reduced radical forms with oxygen, which is a major factor in the shelf-life of these species.

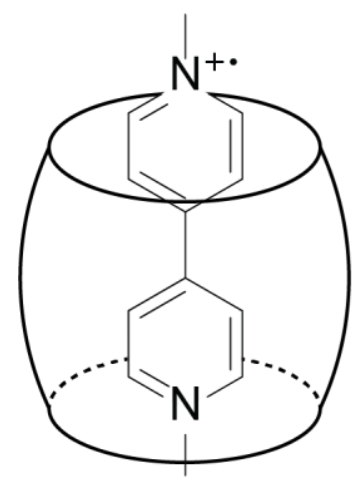

cucurbit[7]uril

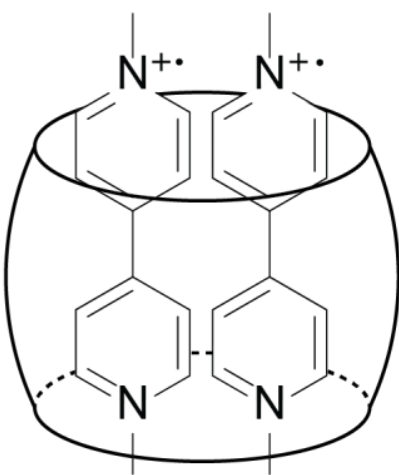

cucurbit[8]uril

Figure 1.10 Encapsulation of methyl viologen radicals by cucurbit[7]uril (left) and cucurbit[8]uril (right).

Little research has been published on slowing the kinetics of viologen radical reaction with $\mathrm{O}_{2}$; however, some reports have manipulated the thermodynamics of viologen radicals. Most of these involve encapsulation inside cage molecules or structures, i.e. host-guest complexation. Molecular encapsulation, or host-guest chemistry, occurs when a large molecule (the host molecule) contains a cavity into which a smaller molecule (the guest molecule) can insert. The insertion is non-covalent, driven by van der Waals forces or entropy. Viologen molecules have been studied primarily as guest molecules using cucurbiturils, cyclodextrins, and 
sulfonatocalixarenes. ${ }^{136-142}$ For example, Figure 1.10 shows encapsulation of methyl viologen radicals inside two different cucurbituril cage molecules: cucurbit[7]uril and cucurbit[8]uril (the numbers 7 and 8 indicate the number of monomers that make up the cucurbituril ring). A single viologen radical molecule can be encapsulated inside cucurbit[7]uril in water. This encapsulation destabilizes the radical by preventing it from pairing with other radicals in solution. The destabilization effect was shown by a negative shift in the reversible electrochemical reduction potential of methyl viologen. ${ }^{143}$ On the other hand, encapsulation of two viologen radical molecules is possible inside the larger cucurbit[8]uril and this leads to stabilization of the radicals by facilitating pairing. ${ }^{142-143}$

\subsection{Scope of thesis}

This thesis can be divided into two separate objectives. The first is the development of RGO thin films and their use for immobilizing a molecular hydrogen evolution electrocatalyst on electrodes for heterogeneous electrocatalysis. The second is the investigation of host-guest complexation between viologen derivatives and cucurbiturils, its effects on viologen reaction with dissolved oxygen, and its implications for long-term storage of reduced viologen solutions.

Chapter 2 discusses a method for fabricating RGO thin films on a variety of surfaces. This method is exceedingly gentle and results in highly reduced, smooth RGO films of controllable thickness. The reduction method is the first published method that uses outer-sphere reductants (cobaltocene derivatives). The films produced by this method are used to adsorb a molecular, redox-active dye to electrodes and are shown to facilitate charge transfer to this molecule.

Chapter 3 investigates the immobilization of the molecular proton reduction catalyst 1 (Figure 1.2) to graphitic thin films on electrode surfaces. Data are presented on three types of graphitic materials: the RGO developed in Chapter 2, commercial single layer graphene, and multiwalled carbon nanotubes (MWCNT). The data show initial adsorption of the catalyst to all three graphitic surfaces, but retention of the physisorbed catalyst was low under turnover conditions except when using an ultra-rough surface. The reason for this was hypothesized to be catalyst intercalation on rough surfaces.

Chapter 4 presents kinetic considerations of proton reduction in an electrocatalytic thin film. The film is composed of RGO with $\mathbf{1}$ embedded among the graphitic layers. The films were prepared by dropcasting mixtures of GO and $\mathbf{1}$ followed by electrochemical reduction. By 
controlling the thickness of the film and the quantity of $\mathbf{1}$, kinetic limitations were determined from cyclic voltammetric data.

Chapter 5 describes the interactions of two bis-viologen derivatives with cucurbit[7]uril (CB[7]) and cucurbit[8]uril (CB[8]) in solution. Both cucurbiturils have high binding constants with both viologens. However, it is shown that $\mathrm{CB}[7]$ thermodynamically destabilizes the reduction of both viologens, whereas $\mathrm{CB}[8]$ stabilizes them. Furthermore, the first 2-electron reduction of each viologen is split into two 1-electron reductions in the presence of $\mathrm{CB}$ [8]. The singly reduced viologen was isolated and is shown to be a unique mono-radical bis-viologen species that has not been observed in solution except in the case of the "blue box molecule" (a cyclic bis-viologen derivative). This mono-radical viologen complexed with $\mathrm{CB}[8]$ is shown here to be significantly stabilized against reaction with dissolved oxygen.

Finally, Chapter 6 discusses preliminary data on five separate fronts and lists future experiments that should be conducted on these projects. These include (1) three methods for covalently grafting small molecules to RGO thin films. These methods can be used to graft a derivative of 1 to electrode surfaces, further improving retention time; (2) in situ XANES data on 1 physisorbed to carbon paper electrodes in neutral and acidic solutions under applied potential; (3) the use of RGO thin films on gallium phosphide photocathodes to immobilize $\mathbf{1}$ for heterogeneous photoelectrocatalytic proton reduction; (4) passivation of silicon photoanodes against oxidative corrosion using RGO thin films; and (5) the formation of self-assembled graphene oxide monolayers on silicon oxide surfaces. 


\section{Chapter 2. Reduction of Graphene Oxide Thin Films by Cobaltocene and Decamethylcobaltocene}

Adapted from ACS Applied Materials and Interfaces, Vol. 10, "Reduction of Graphene Oxide Thin Films by Cobaltocene and Decamethylcobaltocene," MacInnes, M. M.; Hlynchuk, S.; Acharya, S.; Lehnert, N.; Maldonado, S. 2004-2015, Copyright 2018.

\subsection{Introduction}

Thin films of reduced graphene oxide (RGO) are attractive for chemically stable and optically transparent coatings with low electrical resistance. ${ }^{144-146}$ Preparation of thin films of RGO typically involves first the chemical oxidation of graphite to form graphene oxide (GO) suspensions, followed by the casting of GO as a thin film, and finally reductive treatment of the thin film to form RGO. ${ }^{96-97,126}$

To date three main methods for the reduction of $\mathrm{GO}$ thin films to RGO have been investigated. ${ }^{97}$ Electrochemical reduction of GO thin films or electrodeposition of suspended GO particles can be used to form RGO films on electrode surfaces. ${ }^{147-153}$ Applied potentials more negative than $E=-0.6 \mathrm{~V}$ vs. the standard hydrogen electrode (SHE) are generally required to obtain RGO films by this method. The probability that these films incorporate species from the electrolyte is high. ${ }^{154-155}$ In addition, this method is limited only to conductive materials that can serve as electrodes under extreme cathodic potentials. A second method to prepare RGO films is thermal annealing of cast GO films in either a vacuum, inert atmosphere, or reducing atmosphere. ${ }^{97,}$ 156-163 The extent of reductive conversion depends strongly on the atmosphere, time, and temperature. Typically, temperatures in excess of $700^{\circ} \mathrm{C}$ are required, with some researchers recommending temperatures upwards of $1000^{\circ} \mathrm{C} .{ }^{157,162-163}$ This methodology necessarily eliminates the possibility of substrates that cannot withstand such temperatures. A third route is the chemical conversion of GO films to RGO films by reaction with a molecular reductant. A variety of molecular reducing reagents (Table 2.1), including hydrazine, hydroiodic acid, carbohydrates and metal hydrides, have been described. ${ }^{96,120-121,123-126,130,134-135,157,164-178}$ However, these reagents can unintentionally load the RGO films with heteroatoms (e.g. nitrogen, boron, or iodine) ${ }^{96,121,168}$ generate gaseous 
byproducts (e.g. $\mathrm{N}_{2}$ and $\mathrm{H}_{2}$ ) which can perturb the film structure, can be destructive/corrosive to certain substrate materials, and reduce graphene oxide to varying degrees. Other possible methods of reduction include irradiation ${ }^{179}$ and plasma treatment ${ }^{180}$, which each have the potential to damage the substrate material.

\begin{tabular}{|c|c|c|}
\hline Reduction method & Sheet Conductivity $\left(\mathrm{S} \mathrm{m}^{-1} \times 10^{4}\right)$ & Reference \\
\hline \multicolumn{3}{|l|}{ Chemical reductions } \\
\hline Hydrazine monohydrate & 0.02 & 169 \\
\hline Hydrazine monohydrate & 1.6 & 170 \\
\hline Phenylhydrazine & 0.47 & 134 \\
\hline Hydrazine vapor/annealing at $400^{\circ} \mathrm{C}$ & 1.0 & 157 \\
\hline $\mathrm{NaBH}_{4}$ & 0.082 & 120 \\
\hline $\mathrm{NaBH}_{4} / \mathrm{H}_{2} \mathrm{SO}_{4} /$ annealing at $1100^{\circ} \mathrm{C}$ & 2.02 & 120 \\
\hline LiAlH & $<0.01$ & 172 \\
\hline HI & 2.98 & 121 \\
\hline $\mathrm{HI} / \mathrm{AcOH}$ & 3.04 & 126 \\
\hline $\mathrm{HI} / \mathrm{AcOH}$ vapor & 0.785 & 126 \\
\hline Malonic acid & 0.044 & 125 \\
\hline Lawesson's Reagent & 0.476 & 173 \\
\hline Lawesson's Reagent $/ 300^{\circ} \mathrm{C}$ anneal under $\mathrm{H}_{2}$ & 3.09 & 173 \\
\hline $\mathrm{NH}_{3} \mathrm{BH}_{3}$ & 2.03 & 135 \\
\hline Thiourea dioxide/ $\mathrm{NaOH} /$ cholate & 0.3205 & 123 \\
\hline Hydroxylamine/ $\mathrm{NH}_{3}$ & 0.1122 & 174 \\
\hline$p$-phenylene diamine & 1.5 & 175 \\
\hline Isopropyl alcohol & 0.1019 & 130 \\
\hline Benzyl alcohol & 0.46 & 130 \\
\hline L-ascorbic acid & 0.08 & 124 \\
\hline L-ascorbic acid/ $\mathrm{NH}_{3}$ & 0.77 & 176 \\
\hline $\mathrm{NaHSO}_{3}$ & 0.65 & 177 \\
\hline $\mathrm{Na}_{2} \mathrm{~S}_{2} \mathrm{O}_{4} / \mathrm{NaOH}$ & 0.1377 & 178 \\
\hline \multicolumn{3}{|l|}{ Electrochemical Reductions } \\
\hline Electrochemical (-1.3 V vs SCE) & 9.0 & 152 \\
\hline Electrochemical (-0.9 V vs $\mathrm{Ag} / \mathrm{AgCl})$ & 0.85 & 147 \\
\hline \multicolumn{3}{|l|}{ Other Methods of Reduction } \\
\hline Methane plasma & 15.9 & 180 \\
\hline Annealing at $1100^{\circ} \mathrm{C}$ in vacuum & 10 & 157 \\
\hline Annealing at $1000^{\circ} \mathrm{C}$ under $\mathrm{Ar}$ & 8.71 & 162 \\
\hline UV-irradiation under high vacuum & 0.000075 & 179 \\
\hline
\end{tabular}

Table 2.1 Reported methods for the preparation of reduced graphene oxide and the resulting conductivities 
Although isolated reports have demonstrated the interaction between graphene oxide and an electrochemically generated reductant (e.g. methyl viologen radical), ${ }^{181}$ a simple and surprisingly unexplored strategy for producing RGO films is the immersion of GO films in a solution of outer-sphere reductant. Metallocenes are a useful class of outer-sphere reductants. Although metallocene adsorption on nanostructured graphitic carbons has been pursued previously, ${ }^{182-184}$ metallocenes have not yet been explored for the reduction of graphene oxide. Cobaltocene $\left(\mathrm{CoCp}_{2}\right)$ is one possible reductant which has a very negative standard potential $\left(E^{0}=\right.$ $-0.93 \mathrm{~V}$ vs NHE) that ought to facilitate spontaneous injection of $\mathrm{e}^{-}$into $\mathrm{GO}$ and has long been used as a 'clean', one-electron chemical reductant in the synthesis of various inorganic and organic compounds. ${ }^{185}$ The permethylated form, decamethylcobaltocene $\left(\mathrm{CoCp}^{*}\right)$, is an even stronger reducing agent $\left(E^{0}=-1.54 \mathrm{~V}\right.$ vs $\left.\mathrm{NHE}\right)$. Both metallocenes have large self-exchange reaction rates and small reorganization energies for oxidation to the respective metallocenium states, ${ }^{186-187}$ further underscoring their ability to rapidly transfer electrons without substantial bond lengthening/shortening or bond breaking/forming.

Cobaltocene is also interesting since it forms adducts with weak acids when dissolved in aprotic organic solvents. The identity of this adduct is debated, however, and has not received much attention in recent literature. ${ }^{188-190}$ Thus, the reaction of GO with cobaltocene both in the presence and absence of a weak acid like trifluoroacetic acid $\left(\mathrm{p} K_{a}=12.65 \text { in acetonitrile }\right)^{191}$ presents a separate test of whether the reducing strength of the reagent and the availability of protons during reduction imparts a perceptible difference in the resultant RGO films, as suggested by other groups. ${ }^{96}$

Accordingly, in this work, we present data describing the separate reactions of cobaltocene and decamethylcobaltocene with thin GO films, both in the presence and absence of organic acids. This report is the first study to utilize outer-sphere reductants, affording a simpler route to reduce graphitic oxides. We present data that show conclusively that these reactions produce high quality RGO films at room temperature without concomitant mechanical damage to the films or the underlying substrate. Further, we present data that suggests the resultant electrical and chemical properties of the films reduced by the different metallocenes are not equivalent, suggesting it may be possible to produce intentionally RGO films with specific electrical and physicochemical properties. 


\subsection{Experimental.}

Chemicals and Materials. Decamethylcobaltocene (Sigma Aldrich), cobaltocenium hexafluorophosphate (98\%, Sigma Aldrich), trifluoroacetic acid (TFA, 99\%, Sigma Aldrich), methanol (99.9\%, Fisher Scientific) hydrogen peroxide (30\% in $\mathrm{H}_{2} \mathrm{O}$, Sigma Aldrich), hydrochloric acid (37\%, Sigma Aldrich), sulfuric acid (ACS grade, Fisher Scientific Education), tetrabutylammonium perchlorate (G. Frederick Smith Chemical Company), ferrocene (98\%, Acros Organics), aminotriethyoxysilane (98\%, Sigma Aldrich), toluene (anhydrous, 99.8\%, Sigma Aldrich), crystal violet (Sigma Aldrich), hydriodic acid (47-51\%, J.T. Baker Chemical Co.), indium foil (99.99\%, Aldrich), and potassium permanganate (98\%, Acros) were used as received. Cobaltocene (Sigma Aldrich) was purified by sublimation at $T=80^{\circ} \mathrm{C}$. Acetonitrile (99.9\%, Fisher Scientific) and dichloromethane (99.9\%, Fisher Scientific) were distilled and subjected to three freeze-pump-thaw cycles before glovebox use; otherwise solvents were used as received. Graphite flakes (-20+84 mesh, Johnson Matthey) was used as the carbon source for graphene oxide suspensions and for the XPS graphite standard. Water with a resistivity $>18.2 \mathrm{M} \Omega$ (Nanopure Barnstead Water Purification) was used throughout.

Polished Si(111) wafers from Wafer Works doped with As $\left(\leq 0.006 \Omega \mathrm{cm}^{-1}\right)$ were used as substrates for Raman and XPS measurements. Glass microscope slides from Fischer Scientific were used as substrates for UV/vis and conductivity measurements. Fluorine-doped tin oxide (FTO) coated glass from MTI Corporation ( $2 \mathrm{~mm}$ thick, $12-14 \Omega \mathrm{sq}^{-1}$ ) was used for electrochemical measurements. Si wafers, glass slides, and FTO substrates were degreased using a sequence of water, methanol, and acetone, sonicating for ten minutes in each solvent. Glass and FTO were also etched with oxygen plasma (Plasma Etch, PE-50) for 5 minutes. Glassy carbon substrates were prepared by first polishing with alumina $(1 \mu \mathrm{m}, 0.3 \mu \mathrm{m}$, and $0.05 \mu \mathrm{m}$ alumina suspensions $)$ on felt and then soaking in isopropanol for 30 minutes. Indium thin film substrates were prepared by evaporating $600 \mathrm{~nm}$ of indium foil onto degreased glass microscope slides.

Preparation of graphene oxide suspensions. A modified Hummers method was used. ${ }^{192}$ A 4-mL aliquot of water was mixed with $46 \mathrm{~mL}$ of concentrated $\mathrm{H}_{2} \mathrm{SO}_{4}$ and cooled to $T=0^{\circ} \mathrm{C}$ in an acetone bath. After cooling, $1 \mathrm{~g}$ of graphite flakes was added and then $3 \mathrm{~g} \mathrm{KMnO}_{4}$ was added slowly over 1 hour. This suspension was stirred slowly for 48 hours at $T=0^{\circ} \mathrm{C}$. Following, the resultant mixture was a dark green, thick slurry. This suspension was then poured into $300 \mathrm{~mL}$ of chilled water and stirred for another 10 minutes. An $8 \mathrm{~mL}$ aliquot of $30 \% \mathrm{v} / \mathrm{v} \mathrm{H}_{2} \mathrm{O}_{2}(a q)$ was then 
added to quench the reaction. The suspension was filtered with P5 filter paper from Fisher Scientific and the collected materials were re-suspended in 1:9 v/v concentrated $\mathrm{HCl}: \mathrm{H}_{2} \mathrm{O}$. This suspension was then filtered again and re-suspended a total of three times. The wet filtered product was then suspended in pristine water and subjected to dialysis $\left(10,000 \mathrm{~g} \mathrm{~mol}^{-1}\right.$ weight limit) for 2 days. The resultant suspension was then stored in a flask on the benchtop for up to several months.

Preparation of reduced graphene oxide films. A small volume of the graphene oxide suspension was removed and sonicated for 20 minutes to exfoliate and disperse fully individual graphene oxide sheets. This volume was then centrifuged at $9000 \mathrm{rpm}$ for 30 minutes. The yellow supernatant was removed and diluted to approximately $1 \mathrm{mg} \mathrm{mL}^{-1}$. The diluted suspension was then dropcast onto the substrate of choice and allowed to air dry. To obtain uniform films for transmittance measurements, GO suspensions were cast on functionalized glass microscope slides. The glass was first functionalized with aminotriethoxysilane (APTES) by immersion in a $1 \%$ APTES solution in dry toluene for 24 hours followed by thorough rinsing with toluene. The GO suspension was first diluted with ethanol (3:1 ethanol:water) and was then spin casted on the functionalized glass surfaces at $300 \mathrm{rpm}$ for 1 minute followed by $3000 \mathrm{rpm}$ for 3 minutes. This procedure tended to yield films with thicknesses $\sim 1 \times 10^{-8} \mathrm{~m}$. Both drop cast and spin cast GO films were then reduced by immersion in solutions containing either $15 \mathrm{mM} \mathrm{CoCp} 2$ in acetonitrile or $15 \mathrm{mM} \mathrm{CoCp}{ }_{2}{ }_{2}$ in dichloromethane under a dry $\mathrm{N}_{2}$ atmosphere inside a glove box for $30 \mathrm{~min}$ at room temperature. In some experiments, a molar equivalent (with respect to $\mathrm{CoCp}_{2}$ ) of TFA was added to the cobaltocene solution before immersion of the graphene oxide film. Longer times and higher temperatures did not demonstrate change to any reported observable. Following immersion, the films were removed from the glovebox and rinsed with solvent. These films were allowed to air dry under ambient conditions for at least one day before conducting four-point probe measurements. GO thin films on paper and indium metal substrates were also reduced by immersion in HI acid to compare with the reduction methods described here. These samples were immersed in a 50\% v/v solution of HI acid, then removed, and finally rinsed thoroughly with water.

Conductivity measurements Two-point probe measurements were performed by painting two $1 \mathrm{~mm}$ diameter spots of silver print (Sliver Print II from GC Electronics) separated by $2 \mathrm{~mm}$ on the surface of the films deposited on glass slides. Resistance measurements were performed with a TP4000ZC TekPower multimeter (input impedance $=>40 \mathrm{M} \Omega$ ). Separate samples for fourpoint probe measurements were prepared by sputtering gold contacts onto the surface of the films 
using a poly(methyl methacrylate) shadow mask with a grid of $500 \mu \mathrm{m}$ holes spaced by $950 \mu \mathrm{m}$. The sheet resistances were measured using a Keithley 4200 semiconductor parameter analyzer and an Alessi probe station, with the current sourced through the outer two terminals and the voltage drop measured across the inner two terminals. Conductivity values were calculated using thicknesses measured by atomic force microscopy (Figure 2.1). All films for electrical measurements had thickness $\sim 3 \times 10^{-8} \mathrm{~m}$. For more precise measurements, a four-point probe apparatus (Miller FPP-5000) was also used to measure the sheet resistances for these thin films.
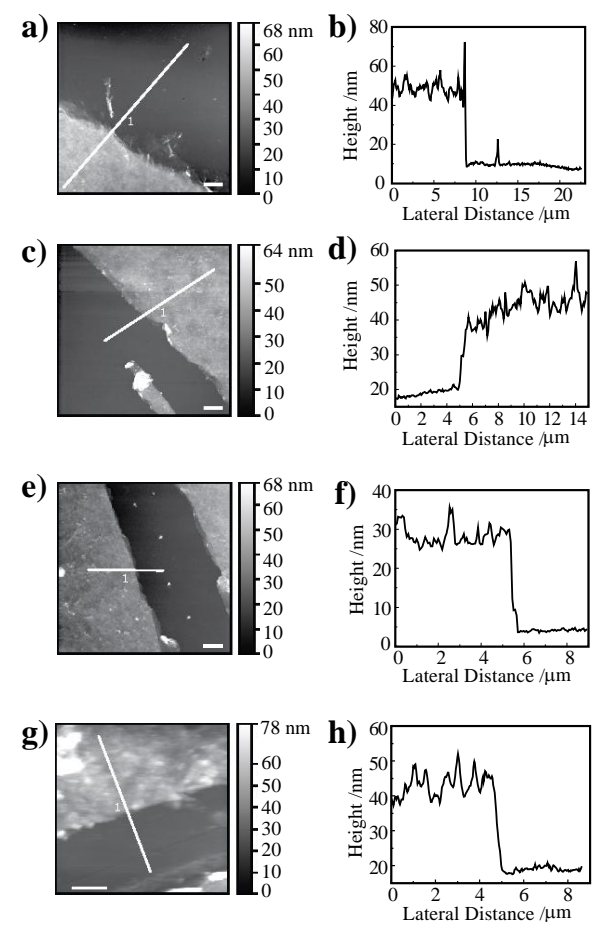

Figure 2.1 Atomic force microscopy (AFM) images and height profiles of representative GO and RGO films used for four-point probe resistivity measurements. AFM image of (a) a GO film on glass, (c) a GO film treated with cobaltocene \& TFA on glass, (e) a GO film treated with decamethylcobaltocene on glass, and (g) a GO film treated with just TFA on glass. Height profiles along the lines denoted as 1 are shown in (b), (d), (f), and (h), respectively. Scale bars: $2 \mu \mathrm{m}$.

$X$-ray photoelectron spectroscopy. X-ray photoelectron (XP) spectra were acquired with a PHI 5400 analyzer using an unmonochromated Al Ka (1486.6 eV) source. Spectra were recorded without charge neutralization at a base pressure of $<2.5 \times 10^{-9}$ Torr. A $6 \mathrm{~mA}$ emission current and $10 \mathrm{kV}$ anode HT was used. Survey scans were acquired at a pass energy of $117.40 \mathrm{eV}$. Highresolution XP spectra were recorded at a pass energy of $23.5 \mathrm{eV}$. Each collected C1s spectra was an average of 100 high resolution scans. 
The C1s spectra were analyzed and fit with the following approach. A C1s spectrum for a pristine graphite flake (Johnson Matthey) was collected. This spectrum was fit with a total of five peaks. The lowest binding energy peak was assumed to correspond to the emitted electrons from carbon atoms involved in the aromatic network of graphite. The peak width and asymmetric tail of this peak were assessed through least-squares fitting and determined to be an asymmetric Gaussian-Lorentzian product function described by the text string "A(0.4,0.38,20)GL(80)" (fit in CasaXPS software) with a peak width of $1.216 \mathrm{eV}$. The other four peaks at higher binding energies were assumed to have symmetric shapes (i.e. these emitted electrons came from carbon atoms not participating in the graphitic $\pi$ electron network) described by a 1:1 convolution of Gaussian and Lorentzian functions and peak widths of 1.5-1.6 eV. The relative positions of all five peaks were based on literature assignments for graphitic carbons and oxidized carbon-containing surface groups ${ }^{193}$ followed by iterative fits of the spectra for the graphitic flake. The specific peak positions used here yielded fits for the spectrum of the graphite flake sample with the lowest fitting residuals and were not allowed to float in the fitting of the data for the thin films. A Shirley background correction was then used for all collected spectra and the lowest energy peak (the asymmetric peak) was calibrated to $284.6 \mathrm{eV}$ in each spectrum.

Raman spectroscopy. Unpolarized Raman spectra were obtained using a Renishaw RM series Raman microscope equipped with a Leica 50x $(N A=0.75)$ objective, with edge filters for the $785 \mathrm{~nm}$ excitation line, in a backscattering geometry. A $785 \mathrm{~nm}$ diode laser was used as the incident excitation source with a total radiant power of $1.12 \mathrm{~mW}$ over a $20 \mu \mathrm{m}^{2}$ spot which was collected for 30 seconds and averaged over 5 scans.

Energy dispersive $x$-ray spectroscopy. Energy dispersive x-ray spectroscopy (EDS) mapping was performed at $5 \mathrm{kV}$ with a PentaFET Precision $\mathrm{x}$-act detector in a LEO 1455VP scanning electron microscope. Films were deposited and treated on clean silicon wafer substrates.

$U V$-visible absorption spectroscopy. Solution UV-visible absorption experiments were performed in $1 \mathrm{~cm}$ quartz cuvettes using an Analytik Jena Specord S600 spectrophotometer. Solid state UV-visible absorption experiments were performed with a Varian Carey 5000 UV-vis-NIR spectrophotometer. Adsorption of crystal violet was performed by immersion of the RGO films on glass in a $50 \mathrm{mM}$ solution of crystal violet in methanol and then rinsing with neat methanol. Spectra represent the absorption of light through dye-loaded films relative to the respective RGO films on glass before adsorption. 
Electrochemical measurements. Electrochemical measurements were taken using a $\mathrm{CH}$ Instruments $760 \mathrm{C}$ potentiostat. A three-electrode cell was used with a supporting electrolyte of deaerated 0.1 M tetrabutylammonium perchlorate and $4 \mathrm{mM}$ trifluoroacetic acid in acetonitrile. A platinum mesh was used as the counter electrode and a $\mathrm{Ag}$ wire immersed in a $\mathrm{AgNO}_{3}$ solution in a fritted compartment was used as a quasi-reference electrode. Absolute potentials were determined by spiking the electrolyte with ferrocene and referencing potentials to the ferrocene/ferricenium couple. Working electrodes were prepared by casting films on FTO-coated glass electrodes.

\subsection{Results.}

Electrical Conductivity of Films. The reaction between metallocenes and graphene oxide films all uniformly resulted in a perceptible color change in the films, as demonstrated in Figure 2.2a. On glass slides, the GO films generally showed a faint, pale yellow color before reduction. The films darkened noticeably within the first $30 \mathrm{~s}$ of immersion, achieving a grey tint. Treatment with just cobaltocene produced films with a very slight grey tint, whereas treatments with cobaltocene \& TFA and with decamethylcobaltocene produced films that were significantly darker. The corresponding transmittance spectra of GO films before and after each treatment are shown in Figure 2.2b.

a)

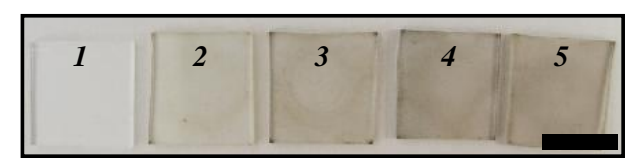

b)

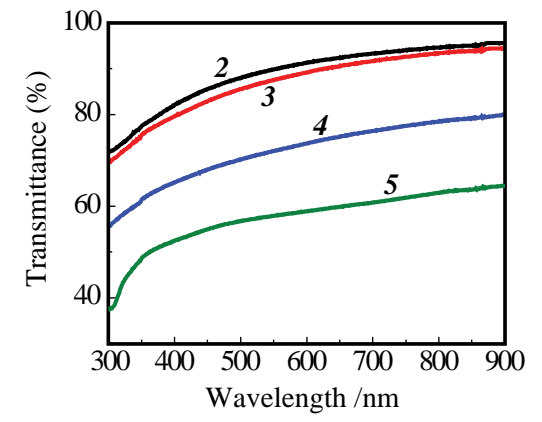

Figure 2.2 a) Photograph of (1) a clean glass microscope slide; (2) a GO film deposited on a glass slide; (3) a GO film on glass treated with cobaltocene; (4) a GO film on glass treated with cobaltocene \& TFA; and (5) a GO film on glass treated with decamethylcobaltocene. b) Corresponding transmittance spectra of the films 2-5 in (a). Scale bar: $1 \mathrm{~cm}$.

Treatment with cobaltocene alone minimally lowered the overall transmittance of the film from that of untreated GO. Treatment with cobaltocene \& TFA further lowered the transmittance, and 
treatment with decamethylcobaltocene produced the darkest films with the lowest transmittance. These films were then measured by a two point probe method for determination of approximate resistance. These values are listed in Table 2.2.

\begin{tabular}{|c|c|c|}
\hline Treatment & $\begin{array}{c}\text { Resistance } / \Omega \\
\text { Two-point probe } \\
\text { measurement }\end{array}$ & $\begin{array}{l}\text { Conductivity /S m-1 a } \\
\text { Four-point probe } \\
\text { measurement }\end{array}$ \\
\hline as-prepared GO & $10^{10}-10^{11 b}$ & $10.2 \pm 0.4$ \\
\hline after reaction with acid & $\geq 10^{13 b, c}$ & -- \\
\hline after reaction with $\mathrm{CoCp}_{2}$ & $(3 \pm 2) \times 10^{5 d}$ & -- \\
\hline after reaction with $\mathrm{CoCp}_{2}+$ acid & $(4 \pm 2) \times 10^{3 d}$ & $(1.2 \pm 0.6) \times 10^{4 e}$ \\
\hline after reaction with $\mathrm{CoCp}_{2}^{*}$ & $(3 \pm 1) \times 10^{3 d}$ & $(1.3 \pm 0.3) \times 10^{4 e}$ \\
\hline after reaction with $\mathrm{CoCp}_{2}{ }_{2}+$ acid & $(1.8 \pm 0.8) \times 10^{3 d}$ & -- \\
\hline
\end{tabular}

Table 2.2 Resistance and conductivities of GO and RGO films prepared under different conditions, measured by four-point probe.

Several points were clear from these data. First, in addition to a color change, all putative RGO films showed a statistically relevant decrease in resistance relative to GO films. Second, the resistance of the putative RGO films was decreased further when a proton source was used in conjunction with cobaltocene to perform the reduction by almost a factor of $10^{-2}$. Although addition of a molar equivalent of TFA to cobaltocene in acetonitrile turned the solution from dark burgundy to dark orange, the absorption spectra indicated that the reaction between cobaltocene and the acid was not stoichiometric, i.e. a large amount of cobaltocene remained unreacted (Figure 2.3). Control measurements where GO films were reacted with TFA solutions in acetonitrile did not result in higher conductivities. Rather, exposure to only this TFA solution consistently increased the measured resistance to a value that exceeded the input impedance of the measuring apparatus, implying acid alone significantly lowered the film conductivity. Third, the presence of a proton donor was not required to render even lower resistance, as immersion in solutions with 

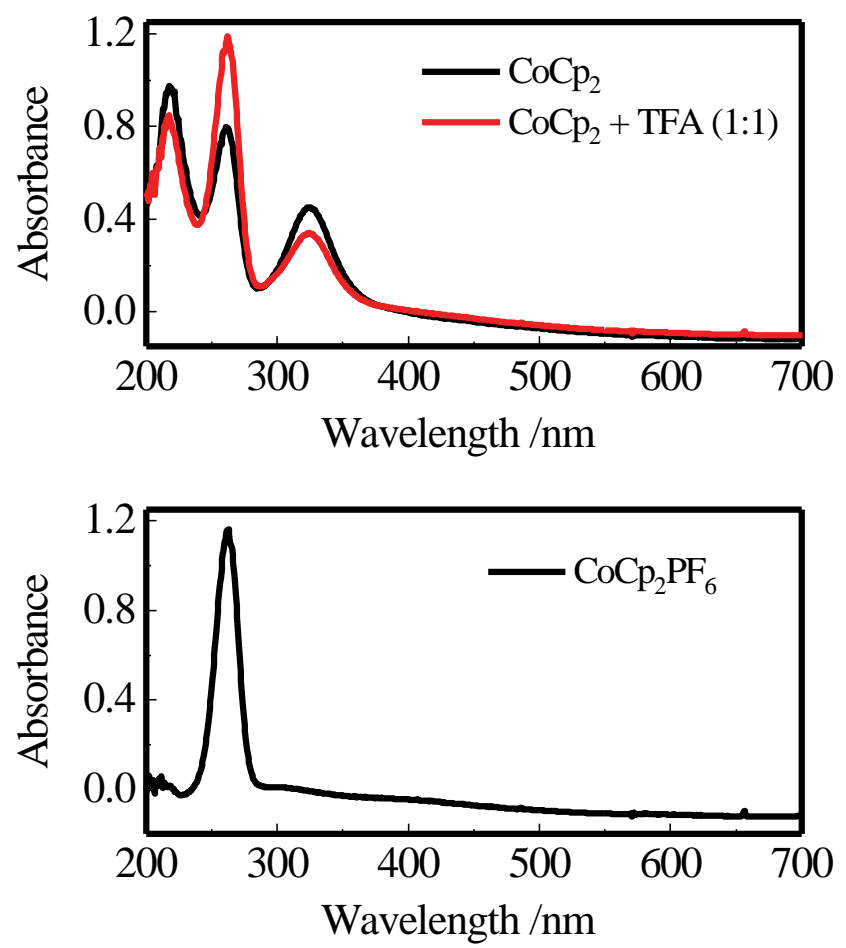

Figure 2.3 (Top) Absorption spectra of cobaltocene in acetonitrile before (black line) and after (red line) addition of one molar equivalent of trifluoroacetic acid. (Bottom) Absorption spectra of cobaltocenium in acetonitrile.

just decamethylcobaltocene showed the lowest resistance. Addition of TFA to these solutions did not produced RGO films with any further decrease in resistance.

Since the reaction of GO films with solutions of either cobaltocene with TFA or just decamethylcobaltocene yielded comparable resistance values, additional four point probe resistance measurements were performed with these putative RGO films to obtain more precise values. Conductivity values were extracted using thicknesses measured by AFM (Figure 2.1). These data are also reported in Table 2.1.

X-Ray Photoelectron Spectroscopy. Figure 2.4 shows representative C1s X-ray photoelectron spectra for a GO film, an RGO film prepared with a solution of cobaltocene, an RGO film prepared with a solution of cobaltocene with TFA, and an RGO film prepared with a solution of decamethylcobaltocene. A conservative fitting approach was adopted, where each spectrum was fit as a linear combination of 5 different peaks (vide supra). The assignments for the five peaks, from lowest to highest binding energy, followed the general conventions historically employed in the XP analysis of graphitic carbons. ${ }^{193}$ These five peaks were ascribed to $s p^{2}$ carbon 
participating in a large aromatic system (i.e. graphitic: 1), $s p^{3}$ carbon bound only to other carbon or hydrogen (2), $s p^{3}$ carbon singly bound to one oxygen (i.e. alcohols and epoxides: 3 ), $s p^{2}$ carbon doubly bound to one oxygen (i.e. quinone, carbonyl: 4), and carbon bound to two oxygens (i.e. carboxylate, ester: 5), respectively. The peak shapes, full widths at half maximum (fwhm), and relative spacings used for fitting these peaks were determined from best fits of the $\mathrm{C} 1 \mathrm{~s}$ spectrum of a clean graphite flake sample. The asymmetric tailing for the graphitic peak (arising from interaction of emitted core electrons with the conduction band) ${ }^{194}$ for this sample was used for the peak shape of the first fitted peak.

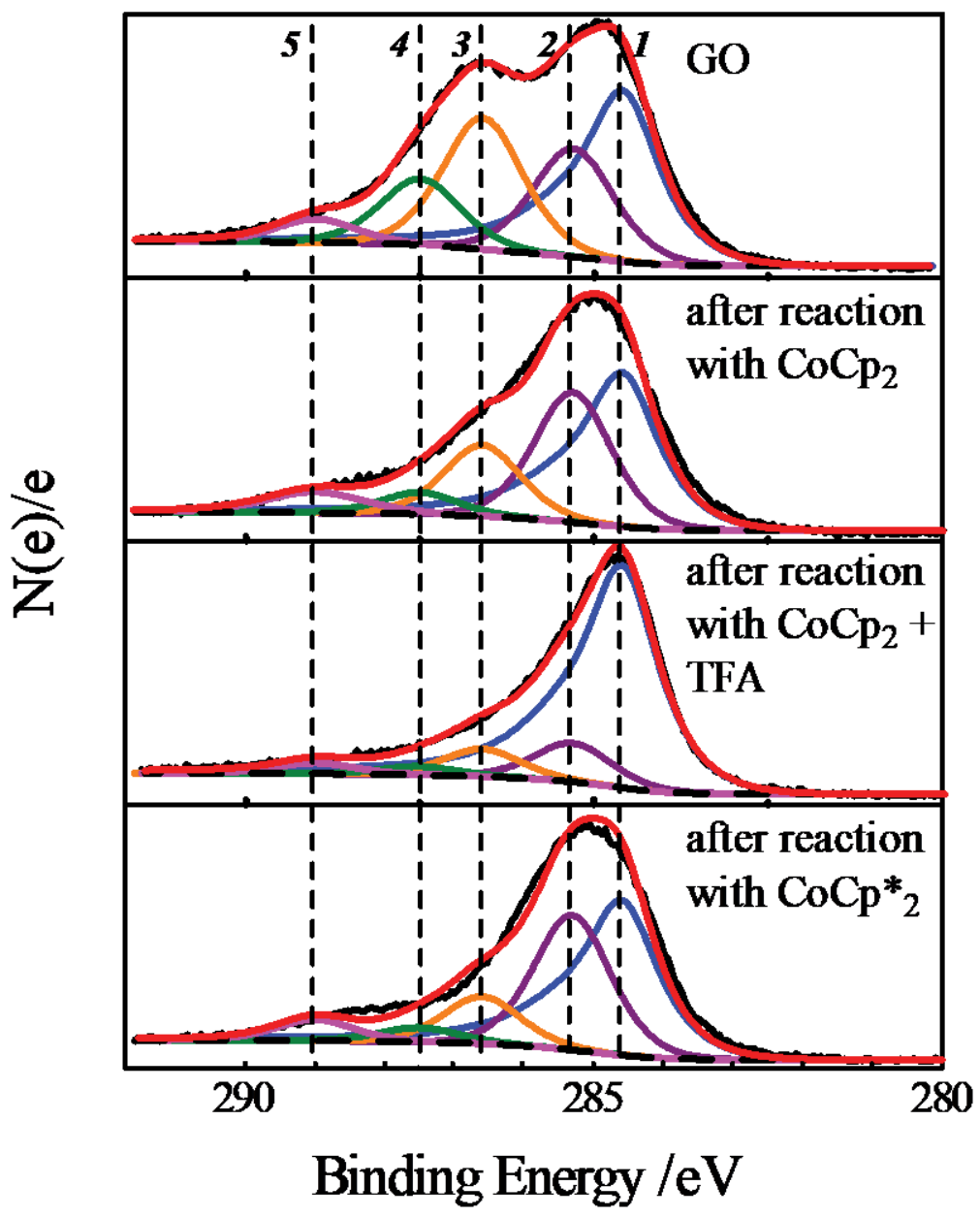

Figure 2.4 Representative high-resolution C 1s X-ray photoelectron spectra of an as-cast GO film and after 30 minute immersions in solutions of cobaltocene, cobaltocene \& TFA, and decamethylcobaltocene, respectively. Thin vertical dashed lines are guides for the eye indicating the binding energies used for the five individual peaks (blue, purple, orange, green, and violet lines) used to generate the fit (solid red line). The backgrounds used to generate the fits are shown as thick dashed lines. 
The results for the fitting analyses of the $\mathrm{C}$ 1s XP spectra are contained in Table 2.3. For GO films, the C1s XP spectrum could be fit with all five peaks, with a sizable contribution from peak 3. RGO films prepared by reaction with cobaltocene yielded differences in the C1s XP spectrum. Peak 1 was still the most pronounced peak but the relative contributions from peaks 3 and 4 were strongly attenuated. The relative intensity of peak 5 was nominally unchanged. The C1s spectrum for RGO films prepared with cobaltocene \& TFA was also different from the data for GO films but in two distinct ways. First, the relative intensity of peak 1 now greatly exceeded the combined contributions from all other peaks. Second, the relative intensity of peak 2 was comparable to the relative intensity of peak 3. In contrast, the C1s spectrum for RGO films prepared with decamethylcobaltocene largely mirrored the spectrum for RGO films prepared with cobaltocene, with slightly smaller contributions from peaks 3,4 , and 5 .

\begin{tabular}{cccccc}
\hline Treatment & 1 & 2 & 3 & 4 & 5 \\
\hline as-prepared $\mathrm{GO}^{b}$ & $38 \pm 7$ & $17 \pm 8$ & $31 \pm 5$ & $9 \pm 3$ & $5 \pm 3$ \\
after reaction with $\mathrm{CoCp}_{2}{ }^{c}$ & $35 \pm 8$ & $40 \pm 9$ & $15 \pm 3$ & $5.1 \pm 0.8$ & $5 \pm 0.9$ \\
after reaction with $\mathrm{CoCp}_{2}+$ acid $^{d}$ & $59 \pm 6$ & $21 \pm 3$ & $10 \pm 2$ & $5.1 \pm 0.8$ & $5 \pm 1$ \\
after reaction with CoCp ${ }^{*}{ }^{e}$ & $33 \pm 6$ & $44 \pm 9$ & $13 \pm 7$ & $5 \pm 1$ & $6 \pm 2$ \\
\hline${ }^{a}$ Peaks labels: 1 - $\mathrm{sp}^{3} \mathrm{C} ; 2-\mathrm{sp}^{2} \mathrm{C} ; 3-\mathrm{C}-\mathrm{O} ; 4-\mathrm{C}=\mathrm{O} ;$ and $5-\mathrm{O}-\mathrm{C}=\mathrm{O} .{ }^{b} N=14,{ }^{c} N=4,{ }^{d} N=7,{ }^{e} N=13$ &
\end{tabular}

Table 2.3 X-ray Photoelectron C 1s Spectra Fitting Results for As-prepared and Reduced Graphene Oxide Films ${ }^{a}$

Figure 2.5 shows the Co 2p XP spectrum of an RGO film prepared with a solution of cobaltocene and trifluoroacetic acid and of an RGO film prepared with a solution of decamethylcobaltocene (red traces). It is apparent that a significant amount of cobalt remains adsorbed to the film surfaces after reduction. EDS analysis in Figure 2.6 also shows residual cobalt signal. After the films have been soaked in an acetonitrile solution of trifluoroacetic acid for two days, however, the cobalt signal is gone (Figure 2.5, red traces). This indicates that the cobalt is weakly adsorbed and can easily be removed. 


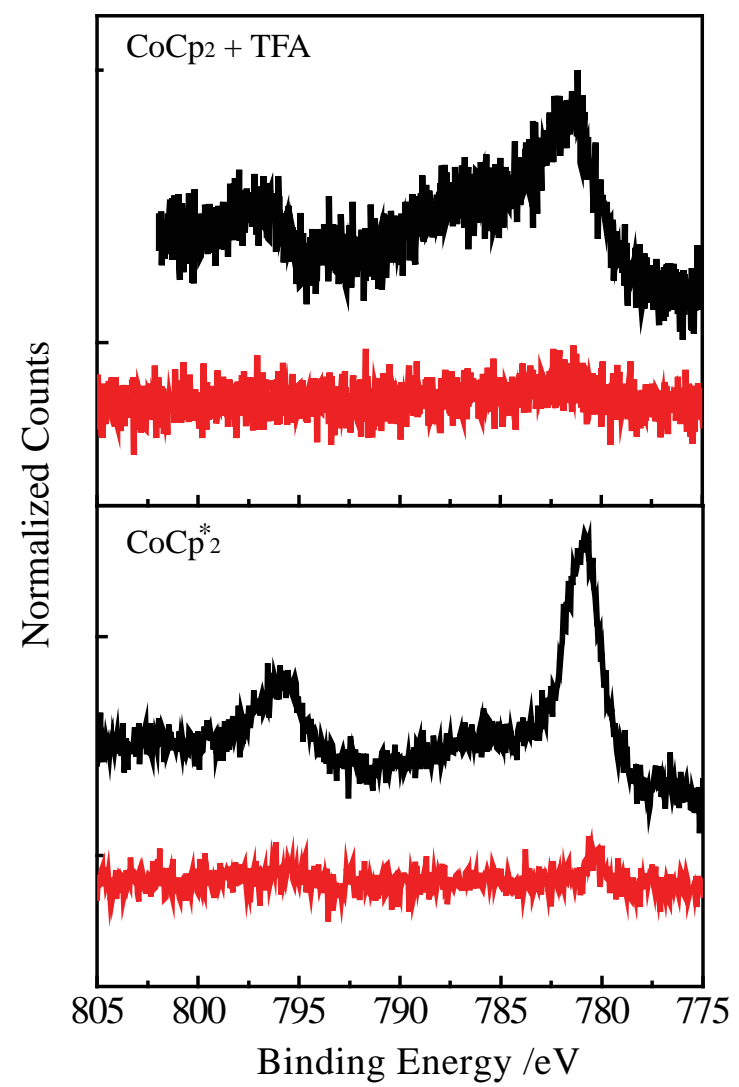

Figure 2.5 Representative Co 2p XP spectra of RGO films treated with cobaltocene \& TFA (top) and films treated with decamethylcobaltocene (bottom). The black lines correspond to freshly prepared RGO films and the red lines correspond to the same films after soaking in the respective solvents for several days. The solvents used for this removal of cobalt species was acidified acetonitrile in the case of films treated with cobaltocene \& TFA and dichloromethane in the case of films treated with decamethylcobaltocene. 


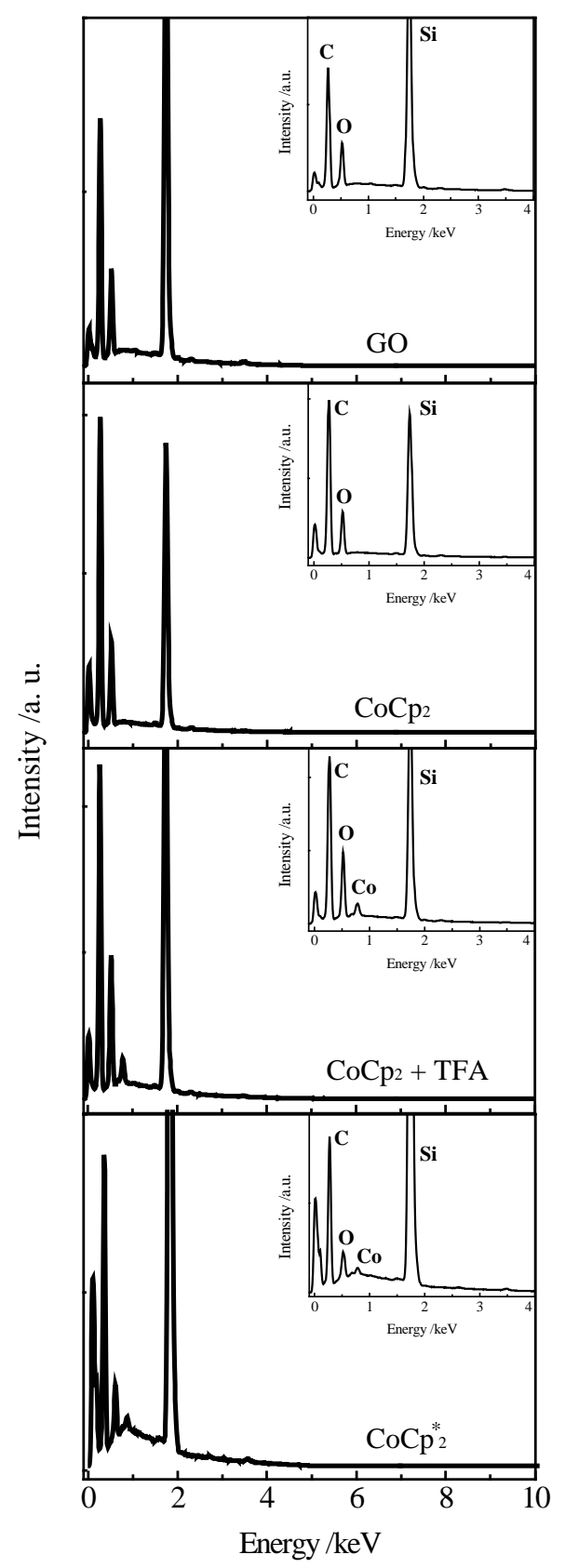

Figure 2.6 Representative energy dispersive x-ray spectra (EDS) of an as-cast GO film on silicon and after 30 minute immersions in solutions of cobaltocene, cobaltocene \& TFA, and decamethylcobaltocene, respectively.

Raman Microscopy. Figure 2.7 summarizes the collected first- and second-order Raman spectra for the GO and RGO film types. Although the loss of symmetry in GO and RGO films can activate several distinct vibrational modes that are otherwise 'silent' in pristine graphite, ${ }^{108-109,195}$ 
no attempt was made here to fit the as-collected spectra with individual modes/“interbands' ${ }^{109}$ Instead, the primary spectral features are described here and summarized in Table 2.4. Each spectrum in Figure 2.7 showed a band near $1320 \mathrm{~cm}^{-1}$ (i.e. the $D$ band) and a second less intense signal near $1610 \mathrm{~cm}^{-1}$ (i.e. the $G$ band). For GO films, the $D$ and $G$ bands were resolvable but showed substantial overlap. Additionally, no G' band (appearing near $2600 \mathrm{~cm}^{-1}$ in graphite) was apparent above the baseline noise. Raman spectra for RGO films prepared with just cobaltocene showed five principal differences. First, the peak positions of the $D$ and $G$ bands red-shifted slightly. Second, the fwhm values for both bands decreased. Third, the overlap between these bands (i.e. the intensity at $\sim 1480-1500 \mathrm{~cm}^{-1}$ ) was less. Fourth, a peak at $\sim 2600 \mathrm{~cm}^{-1}$ (the $2 D$ band) ${ }^{101,106}$ was apparent. The RGO film prepared from a solution of cobaltocene and TFA showed these same changes in the corresponding Raman spectra with some subtle differences. The position of the $D$ and $G$ bands were more red-shifted, the fwhm values for these bands were smaller with lower overlap between the bands, but the relative intensity of the $G$ band remained the same. However, the $2 D$ band at $2600 \mathrm{~cm}^{-1}$ was perceptible over the baseline but with a lower signal to noise ratio. The RGO film prepared with decamethylcobaltocene showed all of these same trends, except $G$, band was clearly visible as well as a secondary peak at $2880 \mathrm{~cm}^{-1}$.

The fifth principal difference in the Raman spectra for the GO and RGO films was the relative intensities of the $G$ band. For graphitic materials, the ratio of the $D$ and $G$ peak intensities (i.e. $I_{D}$ and $I_{G}$, respectively) is proportional to the average crystallite size of a graphitic material, $L_{a}$, but in a complex manner, ${ }^{106-107}$

$$
L_{a}=k\left(\frac{I_{D}}{I_{G}}\right)^{n}
$$

where $k$ is a proportionality constant that depends on the wavelength of excitation, the extent of resonance, the instrument response factor, and the scattering properties of the studied material ${ }^{107}$ and $n$ is the order of the dependence. For graphitic carbons with large (> $3 \mathrm{~nm}$ ) domain sizes, $n$ is 


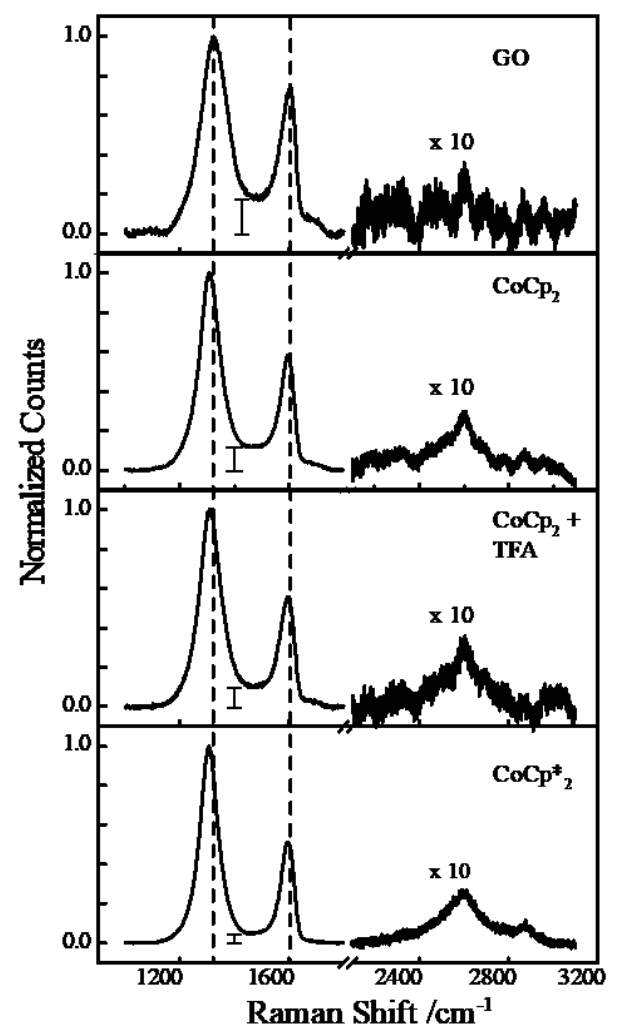

Figure 2.7 Representative Raman spectra of an as-cast GO film and after 30 minute immersions in solutions of cobaltocene, cobaltocene \& TFA, and decamethylcobaltocene, respectively. All raw spectra were normalized to the intensity of the peak near $1320 \mathrm{~cm}^{-1}$. The intensity of the spectra between 2200 and $3200 \mathrm{~cm}^{-1}$ has been increased by an order of magnitude to better visualize the peaks in this region. The scale bar highlights the intensity above the baseline of the overlap region between the $D$ and $G$ peaks.

\begin{tabular}{|c|c|c|c|c|c|}
\hline Treatment & $\begin{array}{c}D \text { band } \\
\text { position } \\
/ \mathrm{cm}^{-1}\end{array}$ & $\begin{array}{l}D \text { band } \\
f w h m \\
/ \mathrm{cm}^{-1}\end{array}$ & $\begin{array}{c}G \text { band } \\
\text { position } \\
/ \mathbf{c m}^{-1}\end{array}$ & $\begin{array}{l}G \text { band } \\
f w h m \\
/ \mathrm{cm}^{-1}\end{array}$ & $I_{D} / I_{G}$ ratio \\
\hline as-prepared GO & $1340 \pm 20$ & $130 \pm 8$ & $1604 \pm 3$ & $70 \pm 20$ & $1.2 \pm 0.2$ \\
\hline after reaction with $\mathrm{CoCp}_{2}$ & $1310 \pm 1$ & $99 \pm 5$ & $1595 \pm 1$ & $71 \pm 8$ & $1.78 \pm 0.07$ \\
\hline after reaction with $\mathrm{CoCp}_{2}+$ acid & $1305 \pm 1$ & $86 \pm 7$ & $1593 \pm 1$ & $63 \pm 6$ & $2.0 \pm 0.2$ \\
\hline after reaction with $\mathrm{CoCp}_{2}^{*}$ & $1306 \pm 1$ & $76 \pm 4$ & $1592 \pm 1$ & $64 \pm 3$ & $2.5 \pm 0.1$ \\
\hline
\end{tabular}

${ }^{a} N=20$ for each measurement; ${ }^{b} \lambda_{\text {laser }}=785 \mathrm{~nm}$

Table 2.4 Raman Spectra for As-prepared and Reduced Graphene Oxide Films ${ }^{a, b}$

-1 , and $e q 1$ is the well-known Tuinstra-König relation. ${ }^{103}$ However, for graphitic materials with very small domains where the crystallite size defines the distance between defects, $n>0$, and that for nanocarbons, $n$ ranges from $\sim 0.75-2 .{ }^{106-107} \mathrm{~A}$ wide range of crystallite sizes for RGO and GO 
has been reported, likely largely as a function of the material preparation method. ${ }^{149,}$ 196-197 The increase in the $I_{D} / I_{G}$ ratio upon treatment with cobaltocenes shown here, which correlates with a decrease in resistivity, implies that the crystallite sizes of these GO and RGO films are $<3 \mathrm{~nm}$. Accordingly, the increase in the $I_{D} / I_{G}$ ratio measured in the RGO films after treatment with the reducing reagents implies a perceptible restoration of the graphitic network in the films. Unfortunately, the exact quantitation of the Raman spectra for graphitic nanocarbons cannot be performed without standards with matched scattering properties and without establishing $n$ beforehand. ${ }^{107}$ Nevertheless, from the spectra in Figure 2.7, RGO films prepared with decamethylcobaltocene showed the largest $I_{D} / I_{G}$ value (Table 2.4), implying that films prepared in this way have larger graphitic domains than films prepared either with just cobaltocene or cobaltocene \& TFA. The proper analysis of Raman spectra from small domain graphitic carbons is an unsettled topic, including the relevant number of bands. ${ }^{106,108-109,195,198-199}$ Some authors prefer to fit the Raman spectra of graphitic carbons with several bands and use the integrated areas of the $D$ and $G$ peaks for the Tuinstra-König analysis; however, because of the disagreement on the proper fitting parameters and number of bands, we limit our analyses here to the ratios of the apparent peak intensities for insight on graphitic character.

Atomic Force Microscopy. Atomic force micrographs were separately collected to ascertain the respective morphologies of the GO and RGO films. Figure 2.8 presents representative atomic force microscopy data showing the topography of each film type. The GO films appeared wrinkled (Figure 2.8a), with an observed root mean square surface roughness of $14 \pm 6 \mathrm{~nm}(N=$ 7). After immersion in a solution of just cobaltocene (Figure 2.8b), the films looked nominally unchanged. The roughness for these films was $10 \pm 2 \mathrm{~nm}(N=7)$. After immersion in solutions with equimolar amounts of cobaltocene \& TFA (Figure 2.8c), the films appeared rougher, with more pronounced wrinkling and a root mean square roughness of $19 \pm 8 \mathrm{~nm}(N=8)$. Reaction of GO films with decamethylcobaltocene (Figure 2.8d) imparted to significant morphological change, but the surface roughness decreased slightly to $8 \pm 3 \mathrm{~nm}(N=7)$.

Substrate/Reagent Compatibility. Figures 2.9 and 2.10 show photographs comparing the results of GO thin film reduction by the most common reductant $(\mathrm{HI}(a q))$ and by cobaltocene \& 

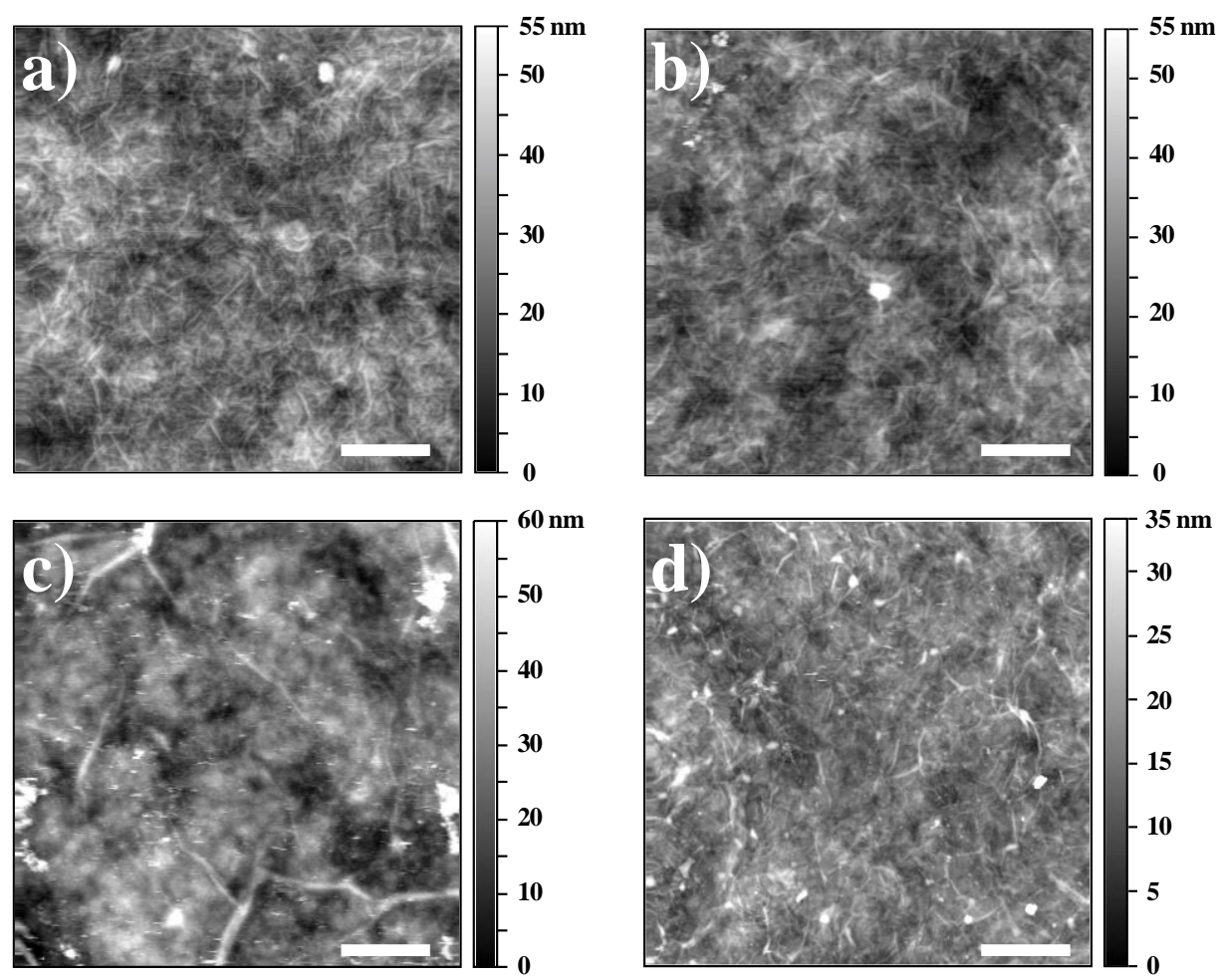

Figure 2.8 Contact mode atomic force micrographs of (a) an as-cast GO film and after 30 minute immersions in solutions of (b) cobaltocene, (c) cobaltocene \& TFA, and (d) decamethylcobaltocene, respectively. Scale bars: $2 \mu \mathrm{m}$.

TFA on two sensitive substrate materials: paper and thin films of indium metal on glass. Figures $2.9 \mathrm{a}$ and $2.9 \mathrm{c}$ show two GO films on indium metal. Figure $2.9 \mathrm{~b}$ shows the sample after immersion in a $\sim 50 \% \mathrm{HI}$ acid solution for 30 seconds. Under these conditions, the GO and indium metal were completely removed from the glass. The inset to Figure $2.9 \mathrm{~b}$ shows the rapid delamination upon immersion in the HI reductant solution. In Figure 2.9d shows the indium/reduced graphene oxide film stack after treatment with cobaltocene \& TFA. The only noticeable change after this treatment was the expected darkening of the graphene oxide film. Otherwise, both the graphitic film and the underlying metal substrate were mechanically unperturbed.

Figures 2.10 and 2.10 show patterned GO films prepared on paper substrates. Figure 2.10b shows the result after immersion in 50\% $\mathrm{HI}(a q)$ solution for 30 seconds, where an intense darkening of both the graphitic film and the paper substrate. The paper was clearly stained by the formation of $I_{2}$ following reaction with the paper. In contrast, Figure $2.10 \mathrm{~d}$ shows the sample in Figure 2.10c after treatment with cobaltocene \& TFA. The only noticeable change after this treatment is the darkening of the now-reduced graphene oxide film pattern. 


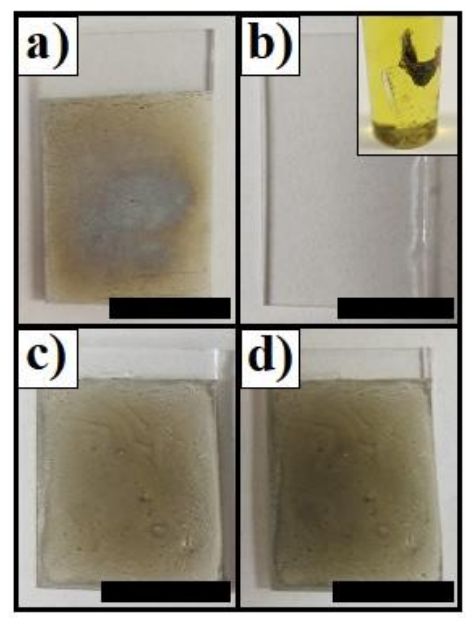

Figure 2.9 a, c) GO films deposited on indium thin films evaporated onto glass. b) The film in (a) after immersion in 50\% HI solution for 30 seconds. d) The film in (c) after treatment with cobaltocene \& TFA. Scale bar: $1 \mathrm{~cm}$.

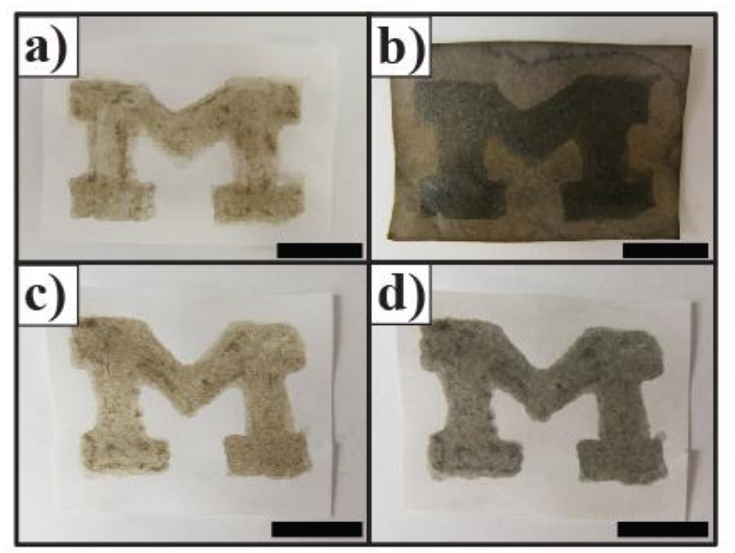

Figure 2.10 (a) and (c) GO films patterned on paper. (b) The film in (a) after immersion in 50\% HI solution for 30 seconds. (d) The film in (c) after treatment with cobaltocene \& TFA. Scale bar: $1 \mathrm{~cm}$

Adsorption on RGO Films as Electrode Coatings. All GO and RGO film types were probed in terms of their efficacies as thin electrode coatings. These film types were prepared on plain glass and FTO-coated glass and then exposed to solutions containing crystal violet for 15 min. Crystal violet is a strongly visible-light-absorbing and redox-active dye. The loading of crystal violet onto the films was monitored through light absorption. Figure 2.11a illustrates that after immersion for $15 \mathrm{~min}$, the GO films showed an order of magnitude higher absorption than any of the RGO films. The RGO film prepared with just cobaltocene had an intermediate absorbance while the other RGO films showed much less light absorbance, implying less extensive dye adsorption. These 
a)

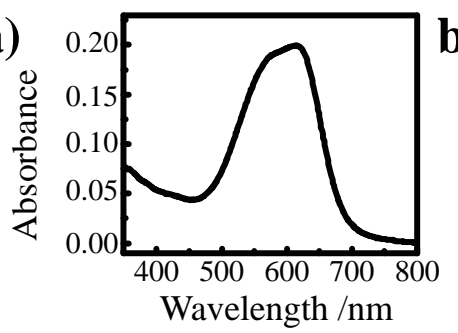

c)

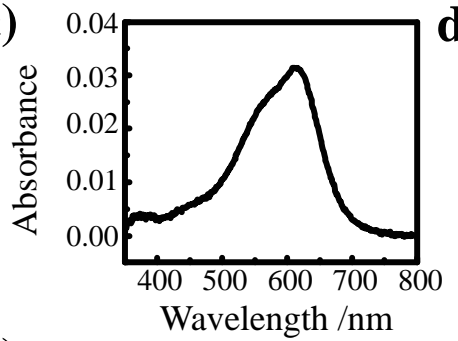

e)

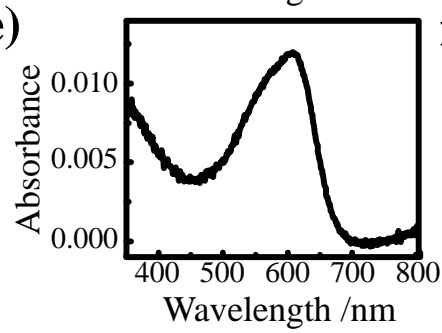

g)

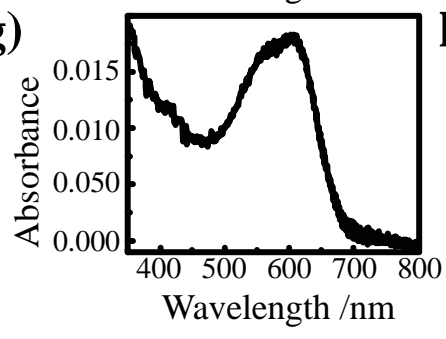

b)

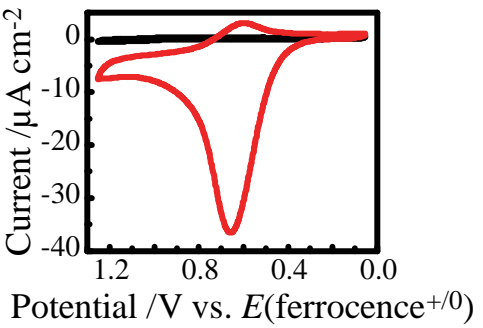

d)

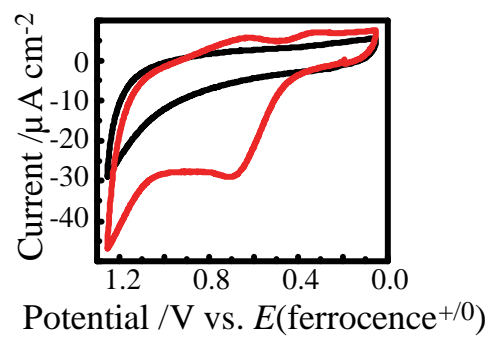

f)

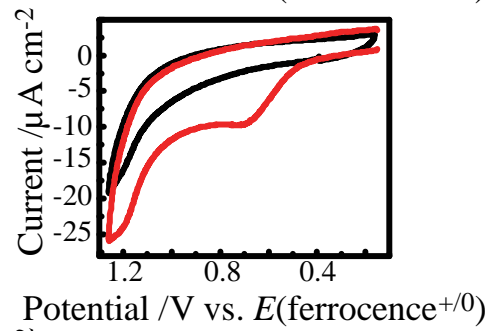

h)

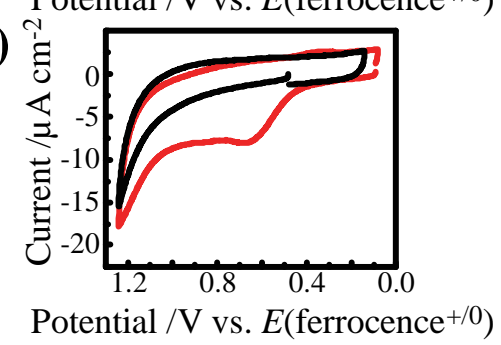

Figure 2.11 Absorption spectra and cyclic voltammetric responses of GO and RGO films after immersion in $50 \mathrm{mM}$ methanol solutions for 15 minutes followed by rinsing with neat methanol. a) Absorption spectrum for crystal violet adsorbed on a GO film and b) the corresponding voltammetric responses before and after crystal violet adsorption. c) Absorption spectrum for crystal violet adsorbed on an RGO film prepared by reduction with cobaltocene and d) the corresponding voltammetric responses for the RGO film before and after crystal violet adsorption. e) Absorption spectrum for crystal violet adsorbed on an RGO film prepared by reduction with cobaltocene and TFA and f) the corresponding voltammetric responses for the RGO film type before and after crystal violet adsorption. g) Absorption spectrum for crystal violet adsorbed on an RGO film prepared by reduction with decamethylcobaltocene and $\mathrm{h}$ ) the corresponding voltammetric responses for the RGO film type before and after crystal violet adsorption. Voltammetric responses were recorded in acetonitrile containing $0.1 \mathrm{M}$ tetrabutylammonium perchlorate and $40 \mathrm{mM}$ TFA. Scan rate: $10 \mathrm{mV} \mathrm{s}^{-1}$.

observations are in accord with the expectation that the cationic dye will have a strong electrostatic affinity with a charged surface and that the GO films had the most anionic character, followed by the cobaltocene-treated RGO films, and then the higher conductivity RGO films had the least anionic character. To assess whether there were any differences in electroactive loadings, cyclic 
voltammograms were recorded with the dye-loaded GO and RGO films, where the potential was biased sufficiently positive to effect the irreversible $2 \mathrm{e}^{-}$oxidation of crystal violet. ${ }^{200}$ Integration of the total charge passed (after correction from the background capacitance) indicated a comparable loading of crystal violet $\left(10^{-9} \mathrm{~mol} \mathrm{~cm}^{-2}\right)$ on the most conductive RGO films, but with a higher loading on the RGO film prepared with just cobaltocene. The shape of the voltammetry was comparable among the three RGO film types, consisting of a double-wave oxidation response, similar to what is seen for solution phase oxidation of crystal violet. ${ }^{200}$ The voltammetric response on the GO film showed only a single wave at approximately the same potential as the first wave in the voltammetry of crystal violet on RGO.

\subsection{Discussion}

The cumulative data support the following contentions. First, graphene oxide can be reduced by $1 \mathrm{e}^{-}$outer-sphere reductants. Second, the standard potential of the reductants strongly influences the extent of reduction. Third, the inclusion of a proton source (acid) in a cobaltocene solution strongly affects the reduction of graphene oxide and the nature of the resultant film. Fourth, the differences in the apparent properties of RGO films prepared by decamethylcobaltocene and by cobaltocene with trifluoroacetic acid do not translate into obvious differences in their electrical or electrochemical properties. These points are discussed below.

Reduction of Graphene Oxide by Outer-Sphere One-Electron Reductants. The substantial and rapid darkening of the as-cast films upon immersion in separate solutions of cobaltocene and decamethylcobaltocene indicate each metallocene readily reacts with graphene oxide. The XP data in Figure 2.4 separately and clearly show that these reactants lowered the overall oxygen content by primarily decreasing the number of alcohol/ether and quinone/carbonyl functionalities, as evidenced by the decrease in peaks 3 and 4, respectively. The Raman data in Figure 2.7 separately suggest a concomitant increase in the overall conjugation in the films, as denoted by the red-shifts of the $D$ and $G$ bands, narrowing of these peaks, and emergence of the $G$ ' band. These facts collectively underscore a restoration of the graphitic $s p^{2}$ network. The atomic force images separately indicate that the chemical reduction effected by reaction with either cobaltocene or decamethylcobaltocene did not substantially alter the physical structure of the graphene oxide films. The surface roughness of GO films decreases upon treatment with each of these reductants, possibly due to an increase in aromaticity that facilitates more layer stacking/ordering. Overall, 
these data are convincing that cobaltocene and decamethylcobaltocene are active for the gentle reduction of GO to RGO, i.e. conversion does not involve elimination of a significant number of carbon atoms.

However, the cumulative data also strongly indicate that the RGO films formed through reaction with these two metallocenes are not equivalent. The lower conductivity of the RGO films consistently produced with cobaltocene implied that this metallocene was less effective at reducing graphene oxide. Further analysis of the XP data argues against the difference in conductivity arising from a lesser ability of cobaltocene to remove oxygen-containing functionalities. Rather, the relative contributions in the $\mathrm{C} 1 \mathrm{~s}$ spectrum from oxygen-containing functionalities in the RGO films made with cobaltocene and decamethylcobaltocene were comparable (Table 2.3). Instead, the Raman spectra argue that removal of oxygen-containing functionalities by cobaltocene did not also restore as much of the conjugated network. That is, reaction with cobaltocene reductively eliminated the same amount of oxygen-containing functionalities as did reaction with decamethylcobaltocene without increasing the conductivity as much. The less prominent band at $\sim 2700 \mathrm{~cm}^{-1}$, the greater overlap between the $D$ and $G$ bands, and the larger Raman shifts for these bands for the RGO films prepared with cobaltocene are all consistent with graphitic domains with less order and smaller sizes. ${ }^{101}$ 103, 105-106, 108-110, 113, 195, 199, 201-202 Accordingly, the data strongly indicate that the less negative standard potential of the cobaltocene/cobaltocenium couple relative to the decamethylcobaltocene/decamethylcobaltocenium couple significantly lessens its ability to effect a conversion of graphene oxide back towards graphene.

The data presented here are the first to implicate an explicit dependence of the physicochemical properties of the resultant RGO films on the reducing power of a chemical reductant. The data clearly suggest that effective reductants do not just remove oxygenfunctionalities but they also must extend conjugation in the films. The presented work does not define what precise electrochemical potential is sufficient to do both, but the value appears to be somewhere between the standard potentials of cobaltocene and decamethylcobaltocene. Given the ability to tune the reducing power of outer-sphere reductants like metallocenes either through change in metal center and/or functionalization of the cyclopentadienyl rings, ${ }^{203-205}$ further experiments may more precisely identify if a single threshold potential exists.

Reduction of Graphene Oxide by Cobaltocene in the Presence of Acid. The reduction of GO films with cobaltocene in the presence of acid was also noticeably different. To be clear, there 
was no evidence of reduction when GO films were exposed to just acid solutions. Rather, the resistance measurements implied the action of acid in the absence of an electron source was to lower the film conductivity. In contrast, the conductivity of RGO films reduced with cobaltocene in the presence of acid was substantially higher. In fact, the conductivity values extracted from four-point probe measurements showed values that were statistically indistinguishable from those of RGO films prepared with decamethylcobaltocene. The XP data clearly implicated that the presence of acid helped further lower the total oxygen-containing functionalities, most specifically the amount of alcohol groups. In addition, the XP data indicated that treatment of GO films with cobaltocene and acid also lowered the apparent amount of $s p^{3}$ carbon (peak 2 in Figure 2.4), suggesting a possible change in the carbon lattice itself. The atomic force micrographs support this premise, with the most substantial roughening of the films occurring after reaction with cobaltocene and acid. The Raman data showed that the spectral features (i.e. peak positions, peak widths, relative intensities) of the films treated with cobaltocene $\&$ acid were different than those for RGO films prepared with just cobaltocene. The Raman spectra indicated that the graphitic domain size after treatment with cobaltocene $\&$ acid fell in between the values for GO films treated with just cobaltocene or just decamethylcobaltocene. However, the overall electrical conductivity of these films was equivalent to that of the films reduced with decamethylcobaltocene. This observation highlights that the sheet conductivity is likely a function of both the graphitic domain size and their interconnectivity. In principle, a material with larger graphitic domains that are electrically isolated from each other (e.g. due to selective functionalization at the periphery of the domains) could show lower macroscopic conductivity. Accordingly, because of the larger $\mathrm{sp}^{2}$ carbon content and lower oxygen content in the RGO films produced with cobaltocene \& TFA as seen in the XP spectra, we posit that the graphitic domains in these films are less 'insulated' by oxygen functionalities at their boundaries than the graphitic domains in films produced with decamethylcobaltocene are. This lower degree of electrical isolation may offset a change in the average graphitic domain size in the RGO films produced with cobaltocene \& TFA, thereby giving these films comparable conductivities to those produced with decamethylcobaltocene. In total, the data indicated that the presence of acid increased the reducing power of cobaltocene for effecting a transformation of GO but with a mechanism different than reduction by decamethylcobaltocene.

Control experiments performed in acetonitrile containing trifluoroacetic acid but without cobaltocene ruled out any specific action of the acid on graphene oxide alone. Rather, the data 
support the contention that a distinct chemical species is involved in solutions containing acid and cobaltocene. The oxidative protonation of cobaltocene in the presence of acids is known (eq 2.2), though the site of protonation (metal center or Cp ligand) is somewhat under debate. ${ }^{188-190}$

$$
\mathrm{CoCp}_{2}+\mathrm{H}^{+} \rightarrow \mathrm{H}-\mathrm{CoCp}_{2}^{+}
$$

Recent electron paramagnetic resonance measurements of decamethylcobaltocene in the presence of triflic acid or lutidinium imply protonation of the $\mathrm{Cp}$ ligand, ${ }^{190}$ suggesting this as the source of activity as a proton-coupled electron transfer reagent.

The mechanism of reduction of GO by this species is not known and cannot be gleaned from the presented data. Nevertheless, the presented evidence is clear on several points. Reduction of GO by this species is more extensive (i.e. substantially lowers oxygen-containing functionalities) than by either metallocene alone while still selective (i.e. does not increase $s p^{3}$ character that would lower conductivity). These features are not common to all metal hydrides. For example, $\mathrm{LiAlH}_{4}$, a strong metal hydride reductant, can reduce GO and almost entirely eliminate oxygen-containing groups. ${ }^{172}$ However, the resultant RGO film conductivities are substantially lower than other RGO film types, ${ }^{172}$ suggesting that $\mathrm{LiAlH}_{4}$ also compromises some of the aromatic network through reduction of $\mathrm{C}=\mathrm{C}$ bonds. In contrast, $\mathrm{NaBH}_{4}$, a milder reductant, also yields RGO films with much lower conductivities (Table 2.1) but with higher residual oxygen content ${ }^{172}$ presumably because it does not reduce GO as extensively. Accordingly, a putative $\mathrm{H}$ $\mathrm{CoCp}_{2}{ }^{+}$species in aprotic solvents may be an intermediate hydride reductant. If so, it might prove useful for selective reductive transformations in organic synthetic chemistry. ${ }^{206}$ Separate work is needed to explore this topic.

Substrate Material Tolerance. The data clearly illustrate the issues that arise when immersion in HI solutions is used to prepare RGO films on sensitive substrates. The corrosion and staining issues are clear with non-noble metals and porous substrates, respectively. These complication negate the high conductivities afforded by reaction with HI (Table 2.1). ${ }^{121}$ 158, 207-208 However, the data presented here demonstrate that metallocene solutions yield comparably conductive RGO thin films without incurring any mechanical or chemical damage to the underlying substrate. In this way, such outer-sphere redox reagents are quite mild with respect to processing considerations while still remaining sufficiently potent reducing reagents. 
RGO Film Coatings. The conductivities of the RGO films prepared by reduction with decamethylcobaltocene and cobaltocene in acid warrant further mention. As detailed in Table 2.1, the majority of RGO films produced by wet chemical reductants yield conductivities $<10^{3} \mathrm{~S} \mathrm{~m}^{-1}$. The conductivities for the RGO films prepared here are slightly higher and are close to the highest conductivities reported for RGO prepared by any method (wet chemical, thermal, or combination). ${ }^{96-97}$ Chemical reductants that yield more conductive RGO films tend to be species that can deliver both electrons and protons. In this manner, the activity of acidic cobaltocene solutions shown here is in line with the potency of other metal hydrides for GO reduction. ${ }^{120,} 172$ However, decamethylcobaltocene is unique, as it can only deliver electrons. Regarding reductants, its closest analog is not molecular but rather the approach to electrochemically reduce GO films cast on metallic electrodes. ${ }^{147-148,150,155}$ The data are also clear that the RGO films exhibit electrochemical responses for a probe cationic adsorbate that are sensitive to the extent of reduction in the films. We posit that the ionic character of GO films are not equally diminished upon reduction by all the investigated reducing solutions. More work is needed to determine whether the resultant electrical and electrochemical properties are not sensitive to just variations in surface chemistry/ordering. The use of other outer-sphere reductants and correlating the resultant properties/behaviors of the RGO films may inform on this topic.

\subsection{Conclusions.}

This work demonstrates that it is possible to reduce graphene oxide thin films by simple immersion in non-aqueous solutions containing strongly reducing metallocenes. Cobaltocene and decamethylcobaltocene are specifically shown to be active in reducing the number of oxygencontaining functionalities in and affecting the graphitic domain size of graphene oxide. The data also show that decamethylcobaltocene is more effective at reducing graphene oxide, suggesting a strong dependence on the standard potential of the reductant. This difference could be offset somewhat if cobaltocene was used in conjunction with a proton source during the reduction of GO films. These resultant RGO films show similarly high electrical conductivities, on par with films prepared by just decamethylcobaltocene. This work motivates further studies on two fronts. From a practical point of view, the results demonstrate a simple, effective, and very mild means to prepare highly conductive RGO films on any substrate type (insulator, semiconductor, conductor), even on substrates that are sensitive to corrosion or impregnation. This work importantly 
demonstrates reduction of graphene oxide by outer-sphere reductants for the first time, thereby highlighting a new way to explore and understand the reduction of graphene oxide materials through the tunability of outer-sphere reductants. 


\section{Chapter 3. Physisorption of a Molecular Proton Reduction Electrocatalyst on Thin Graphitic Films}

\subsection{Introduction}

In the face of an ever-increasing global energy demand and a looming environmental crisis due in part to emissions from carbon-based fuels, electrochemical water splitting is a promising method for generating a clean chemical fuel. In electrochemical water splitting, water is converted to hydrogen gas (at the cathode) and oxygen gas (at the anode). The hydrogen evolution halfreaction can be driven at its thermodynamic potential using a platinum electrode, but because of platinum's scarcity and costliness, it is preferable to use other, cheaper electrode materials. Most of these materials, however, require significant overpotentials due to unfavorable reaction kinetics at the electrode surface. The required overpotential can be lessened by addition of an electrocatalyst to the system. To this end, a wide range of proton reduction electrocatalysts have been developed. These include solid state materials, such as metal sulfides or oxides ${ }^{38}$, and molecular complexes, like the famous nickel diphosphine catalyst ${ }^{209}$ among others. ${ }^{32,210-211}$ Solid state catalysts have the benefit of operating heterogeneously, i.e. immobilized on the electrode surface, whereas molecular catalysts are most often homogeneous, i.e. dissolved in the electrolyte solution. Heterogeneous catalysis results in far less material waste than homogeneous catalysis. Molecular catalysts, however, have the advantage of a well-defined and easily tunable active site. It is therefore advantageous to find ways of heterogenizing molecular electrocatalysts, thereby combining the benefits of both types of systems.

Molecular catalysts can be immobilized on electrode surfaces in two ways: through physisorption by utilizing attractive van der Waals forces $54,60,91,212-213$ or by covalent attachment. ${ }^{34,214-216}$ Physisorption has the benefit of simplicity and ease of execution. One of the most common van der Waals forces used for physisorption on surfaces is $\pi$ - $\pi$ stacking interactions, which can occur between a molecule containing a $\pi$-system that can lie flat on a surface and a 
surface that also contains a $\pi$-system, e.g. graphitic carbon surfaces. The system discussed in this chapter utilizes these attractive forces.

Herein we report on the physisorption of the molecular proton reduction electrocatalyst cobalt (III) bis(dichlorobenzenedithiolate) (1) (Figure 1.2) to graphitic thin films on electrode surfaces. A set of cobalt bis(benzenedithiolate) proton reduction catalysts has previously been studied for their physisorption to graphite-based electrodes ${ }^{69,} 217$ and electrodeposited reduced graphene oxide (RGO) on FTO electrodes. ${ }^{218}$ Long-term stability during electrolysis in acidic conditions was demonstrated on graphite electrodes. Graphite is a low-cost, stable material commonly used for inert electrodes. However, to implement this system in photoelectrochemical cells for solar driven water-splitting, transparent graphitic thin films are required. To this end we report on the adsorption of $\mathbf{1}$ to three different transparent graphitic thin films: RGO, single layer graphene, and multiwalled carbon nanotubes (MWCNT). While it has previously been shown that adsorption to an electrodeposited film of RGO on an FTO surface leads to stable attachment, ${ }^{218}$ the data presented here suggest that adsorption to dropcast RGO films, graphene, and MWCNT films is extremely weak and desorption occurs quickly under turnover conditions. Furthermore, casting a film of a proton-conductive polymer on top of the adsorbed catalyst layer does not improve retention of the catalyst. The reason for the weak adsorption of catalyst on these surfaces is hypothesized to be the ultra-low roughness of the surfaces, preventing any intercalation within the film. To support this hypothesis, voltammetry data are presented, which show that increasing the roughness of the RGO film does indeed improve catalyst retention.

\subsection{Experimental}

Materials. Trifluoroacetic acid (TFA, 99\%, Sigma Aldrich), hydrogen peroxide $\left(30 \%\right.$ in $\mathrm{H}_{2} \mathrm{O}$, Sigma Aldrich), hydrochloric acid (37\%, Sigma Aldrich), sulfuric acid (ACS grade, Fisher Scientific Education), dimethylformamide (99.8\%, Sigma Aldrich), multi-walled carbon nanotubes (>98\% carbon basis, O.D. $\times$ L 6-13 $\mathrm{nm} \times 2.5-20 \mu \mathrm{m}$, Sigma Aldrich), Nafion (5\% wt. in lower aliphatic alcohols and water, Sigma Aldrich), graphite rods (Graphite Machining, Inc. Grade NAC-500 purified, <5 ppm ash level), and potassium permanganate (98\%, Acros) were used as received. Cobaltocene (Sigma Aldrich) was purified by sublimation at $T=80^{\circ} \mathrm{C}$. Acetonitrile (99.9\%, Fisher Scientific) was distilled and subjected to three freeze-pump-thaw cycles before glovebox use; otherwise solvents were used as received. Potassium hexafluorophosphate (95\%, TCI) was recrystallized from aqueous $0.1 \mathrm{M} \mathrm{KOH}$ before use. 
Graphite flakes (-20+84 mesh, Johnson Matthey) were used as the carbon source for graphene oxide suspensions. Single layer graphene on copper (Graphenea) was transferred to the desired substrate with thermal tape. Water with a resistivity $>18.2 \mathrm{M} \Omega$ (Nanopure Barnstead Water Purification) was used throughout.

Polished Si(111) wafers from Wafer Works doped with As $\left(\leq 0.006 \Omega \mathrm{cm}^{-1}\right)$ were used as substrates for XPS measurements. Glassy carbon substrates were prepared by first polishing with alumina ( $1 \mu \mathrm{m}, 0.3 \mu \mathrm{m}$, and $0.05 \mu \mathrm{m}$ alumina suspensions) on felt and then soaking in isopropanol or methanol for 5 minutes.

Preparation of graphene oxide suspensions. See Chapter 2.2 Experimental for preparation of graphene oxide.

Preparation of RGO films. A small volume of the graphene oxide suspension was removed and sonicated for 20 minutes to exfoliate and disperse fully individual graphene oxide sheets. This volume was then centrifuged at $9000 \mathrm{rpm}$ for 30 minutes. The brown supernatant was removed and diluted to approximately $1 \mathrm{mg} \mathrm{mL}^{-1}$. The diluted suspension was then dropcast onto the substrate of choice and allowed to air dry. These films were then reduced by immersion in solutions containing $15 \mathrm{mM} \mathrm{CoCp} 2$ and $15 \mathrm{mM}$ TFA in acetonitrile under a dry $\mathrm{N}_{2}$ atmosphere inside a glove box for $30 \mathrm{~min}$ at room temperature. Following immersion, the films were removed from the glovebox and rinsed with solvent. These films were allowed to air dry under ambient conditions.

Preparation of MWCNT films. About $1 \mathrm{mg}$ MWCNTs were suspended in about $2 \mathrm{~mL}$ DMF and sonicated for $\sim 15$ minutes. The suspension was centrifuged briefly to remove large particles. The resulting gray colloid was stable for several weeks. This colloid was dropcast on a desired surface (about $50 \mu \mathrm{L}$ per $\mathrm{cm}^{2}$ surface area) and allowed to air dry in ambient conditions.

Preparation of freeze-dried RGO (fd-RGO) films. Graphene oxide (GO) suspension (about $0.1 \mathrm{~mL}$ of $1 \mathrm{mg} / \mathrm{mL}$ suspension cast per $1 \mathrm{~cm}^{2}$ surface area) was dropcast on the desired surface and allowed to air dry. A similar sized droplet of GO was then cast on top of this film and the sample was immediately immersed in liquid nitrogen. Note that the underlying GO thin film was necessary when preparing samples on glassy carbon substrates because the wetting of GO on glassy carbon was so poor that the frozen GO droplet tended to easily slide off the substrate. If a thin film of GO was first prepared on the glassy carbon, then the frozen GO droplet adhered to the surface much more strongly. Immediately after freezing, the sample was then subjected to lyophilization. Once dried, the film was light brown and had a soft appearance. The GO was 
reduced to RGO by cobaltocene following the same procedure as described above for smooth RGO films.

Synthesis of 1 . In an inert atmosphere box under $\mathrm{N}_{2}$ pressure (Innovative Technologies), a solution of 3,6-dichloro-1,2-benzenedithiol (0.5 g, $2.4 \mathrm{mmol})$ and sodium methoxide (0.128 g, 2.4 mmol) was added dropwise to a suspension of cobalt(II) sulfate heptahydrate $(0.320 \mathrm{~g}, 1.14 \mathrm{mmol})$ in $40 \mathrm{~mL}$ of dry methanol in a roundbottom flask. The addition caused a color change to deep blue. The resultant solution was stirred for 2 hours. A solution of tetrabutylammonium bromide $(0.407$ $\mathrm{g}, 1.2 \mathrm{mmol}$ ) in $6 \mathrm{~mL}$ dry methanol was added at this time, and the solution was stirred for an additional 2 hours. The sealed flask was then removed from the glovebox and the solvent volume was reduced under vacuum to $<10 \mathrm{~mL}$, resulting in a thick, dark blue slurry. This was filtered and dried. The solid was then recrystallized from dichloromethane/ether to yield $\mathbf{1}$ as a dark blue crystalline solid (50\% yield). The UV-visible absorption spectrum matched that in the literature. ${ }^{218}$

Adsorption of 1 to carbon surfaces and Nafion capping layer. To adsorb 1, all films were immersed in a $5 \mathrm{mM}$ solution of $\mathbf{1}$ in acetonitrile for at least 30 minutes, then removed and rinsed gently but thoroughly with acetone. They were then gently dried with a stream of nitrogen.

To form a capping layer of Nafion, about $50 \mu \mathrm{L}$ of a Nafion solution per $\mathrm{cm}^{2}$ of surface area was dropcast on top of the carbon thin films. The Nafion capping layer was cast after adsorbing 1 to the carbon surface.

Preparation of graphite surface for AFM. Graphite rods were polished with coarse sandpaper to expose a fresh surface, then with fine sandpaper, and finally with alumina polish $(0.05 \mu \mathrm{m})$, according to the procedure in Reference 217 . The end was then sawed off with a diamond saw to make a puck that was placed face-up on the AFM stage.

$X$-ray photoelectron spectroscopy. X-ray photoelectron (XP) spectra were acquired with a PHI 5400 analyzer using an unmonochromated Al Ka (1486.6 eV) source or a Kratos Axis Ultra analyzer using a monochromated Al Ka (1486.6 eV) source. Spectra recorded on the PHI instrument were taken without charge neutralization at a base pressure of $<2.5 \times 10^{-9}$ Torr. A $6 \mathrm{~mA}$ emission current and $10 \mathrm{kV}$ anode HT was used. High-resolution XP spectra were recorded at a pass energy of $23.5 \mathrm{eV}$. Each C1s spectrum collected is an average of 20 high resolution scans and each other element spectrum collected is an average of 50 high resolution scans. 
Spectra recorded on the Kratos instrument were taken with charge neutralization at a base pressure of $<2.5 \times 10^{-9}$ Torr. An $8 \mathrm{~mA}$ emission current and $14 \mathrm{kV}$ anode HT were used. Each high-resolution spectrum taken was an average of 10 scans at a pass energy of $20 \mathrm{eV}$.

All spectra were calibrated to the $\mathrm{C} 1 \mathrm{~s}$ peak at $284.6 \mathrm{eV}$.

Electrochemical measurements. Electrochemical measurements were taken using a $\mathrm{CH}$ Instruments $760 \mathrm{C}$ potentiostat. A three-electrode cell was used with a supporting electrolyte of deaerated 0.1 M potassium hexafluorophosphate and various concentrations of trifluoroacetic acid in water. Platinum mesh was used as the counter electrode and $\mathrm{Ag} \mid \mathrm{AgCl}$ (sat'd $\mathrm{KCl}$ ) (in a compartment separated by a glass frit) was used as the reference electrode. RGO, MWCNT, and single layer graphene films were cast or transferred, respectively, on glassy carbon disk electrodes with a diameter of $3 \mathrm{~mm}$. The fd-RGO films were cast on square plates of glassy carbon which were then compressed in an o-ring cell for electrochemical analysis. The region of exposed electrode in this cell was $4.47 \mathrm{~mm}$ in diameter.

Atomic force microscopy. AFM was conducted on an Anasys Instruments NanoIR2. Images were taken with $300 \mathrm{pt}$ resolution in both $\mathrm{x}$ and $\mathrm{y}$ directions. Images of RGO thin films and polymer coated films were taken at $1 \mathrm{~Hz}$ in contact mode. Images of fd-RGO films were taken at $0.5 \mathrm{~Hz}$ in tapping mode.

Fourier transform infrared spectroscopy. FTIR spectroscopy was performed on a Thermo Scientific Nicolet iS50 instrument with an ATR diamond crystal attachment. Graphene oxide suspensions were dropcast on the ATR crystal and allowed to air dry for several minutes before obtaining a spectrum.

Scanning electron microscopy. SEM images were taken on a Zeiss LEO 1455VP microscope under $5 \mathrm{kV}$ accelerating voltage and $10 \mathrm{pA}$ beam current.

\subsection{Results}

Adsorption to surfaces. Figure 3.1 shows the Co 2p XP spectra of the three types of carbon thin films (excluding freeze-dried (fd)-RGO) after soaking in a $5 \mathrm{mM}$ solution of $\mathbf{1}$. The strong Co $2 \mathrm{p}$ signal indicates adsorption of $\mathbf{1}$ to all surfaces. It should be noted that the spectrum of RGO/1 was taken on an instrument lacking a monochromator whereas the spectra of graphene/1 and MWCNT/1 were taken on an instrument with a monochromated x-ray source. This is the reason for the difference in peak widths and signal-to-noise ratio among the three spectra. There is a 
distinct shift in the binding energy of the cobalt signal among the three materials. The Co $2 \mathrm{p}_{3 / 2}$ peaks of graphene/ $\mathbf{1}$ and MWCNT/1 occur at a binding energy of approximately $779.4 \mathrm{eV}$ and $780.0 \mathrm{eV}$, respectively, whereas the same peak occurs at significantly higher energy, $781.8 \mathrm{eV}$, for $\mathrm{RGO} / \mathbf{1}$. Furthermore, a second peak appears in the $\mathrm{RGO} / \mathbf{1}$ spectrum at around $787 \mathrm{eV}$.

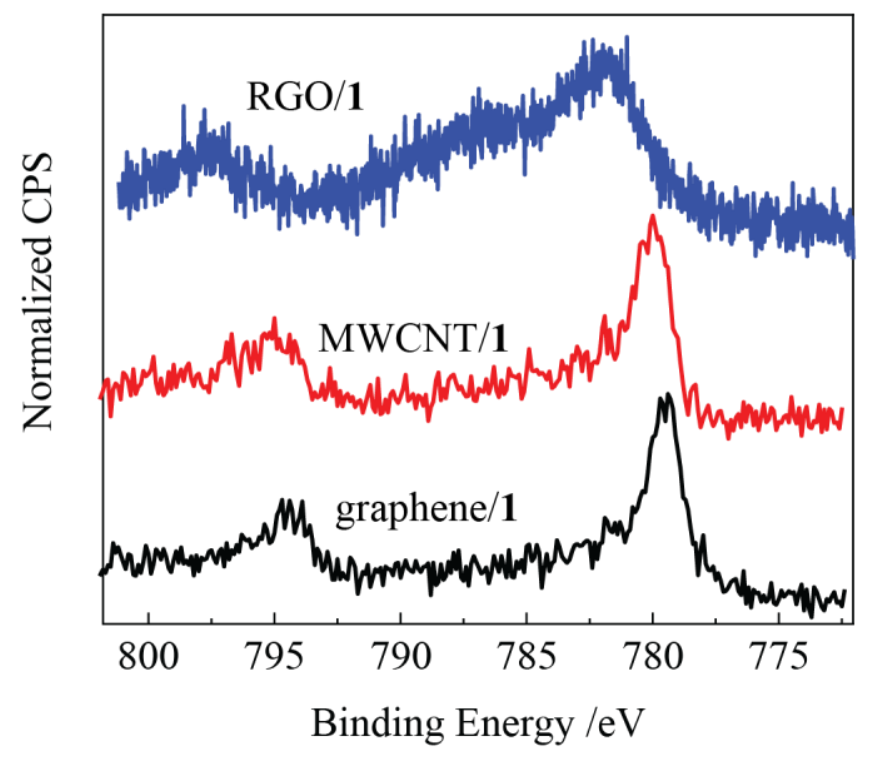

Figure 3.1 Co 2p XP spectra of RGO (blue), MWCNT (red), and graphene (black) after soaking in a $5 \mathrm{mM}$ solution of $\mathbf{1}$.

Voltammetry with acid titrations. Figure 3.2 shows the voltammetry of each type of carbon surface with 1 adsorbed, with and without a Nafion film, and with varied trifluoroacetic acid concentrations (up to at least $4 \mathrm{mM}$ ). Two observations from this voltammetry can be made. The first is that for RGO/1, graphene/1, and MWCNT/1 by at least $8 \mathrm{mM}$ acid the catalytic peak begins to disappear and shift to more negative values. This trend is not observed for fd-RGO/1. In this case, the catalytic peak is stable up to $8 \mathrm{mM}$ acid concentrations. Further experiments with fd$\mathrm{RGO} / \mathbf{1}$ in $10 \mathrm{mM}$ and $50 \mathrm{mM}$ acid concentrations showed stability over many cycles (Figure 3.3). Secondly, for each carbon thin film material (RGO, graphene, and MWCNT), the onset of catalytic current occurs at a more positive potential with a Nafion capping layer than without Nafion. The dashed gray lines are guides for the eye to show the shift in onset potential between films with Nafion and films without Nafion. Notably, the current densities at $2 \mathrm{mM}$ acid (before catalysis starts to drop off) are approximately the same for all materials. 


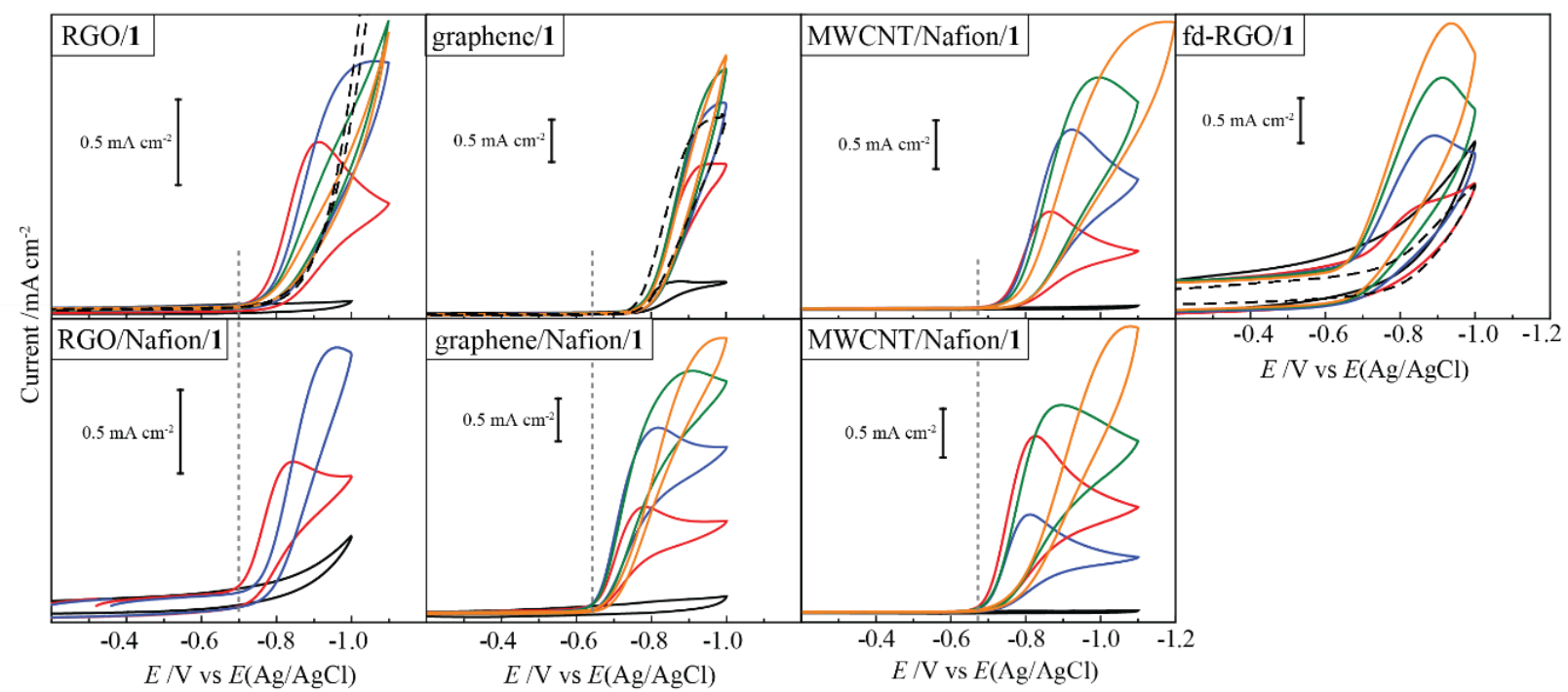

Figure 3.2 Cyclic voltammetry of various graphitic thin films after soaking in a $5 \mathrm{mM}$ solution of $\mathbf{1}$ in acetonitrile for $>1 \mathrm{hr}$ with (top) and without (bottom) a Nafion capping layer. Supporting electrolyte was deaerated $0.1 \mathrm{M} \mathrm{KPF}_{6}$ (black), with $2 \mathrm{mM}$ TFA (red), $4 \mathrm{mM}$ TFA (blue), $6 \mathrm{mM}$ TFA (green), or $8 \mathrm{mM}$ TFA (orange). The dashed black curves are graphitic surfaces with no 1 adsorbed in $8 \mathrm{mM}$ TFA. The vertical dashed lines are guides for the eye to show shift in onset of current with a Nafion capping layer. The scan rate was $50 \mathrm{mV} / \mathrm{s}$.
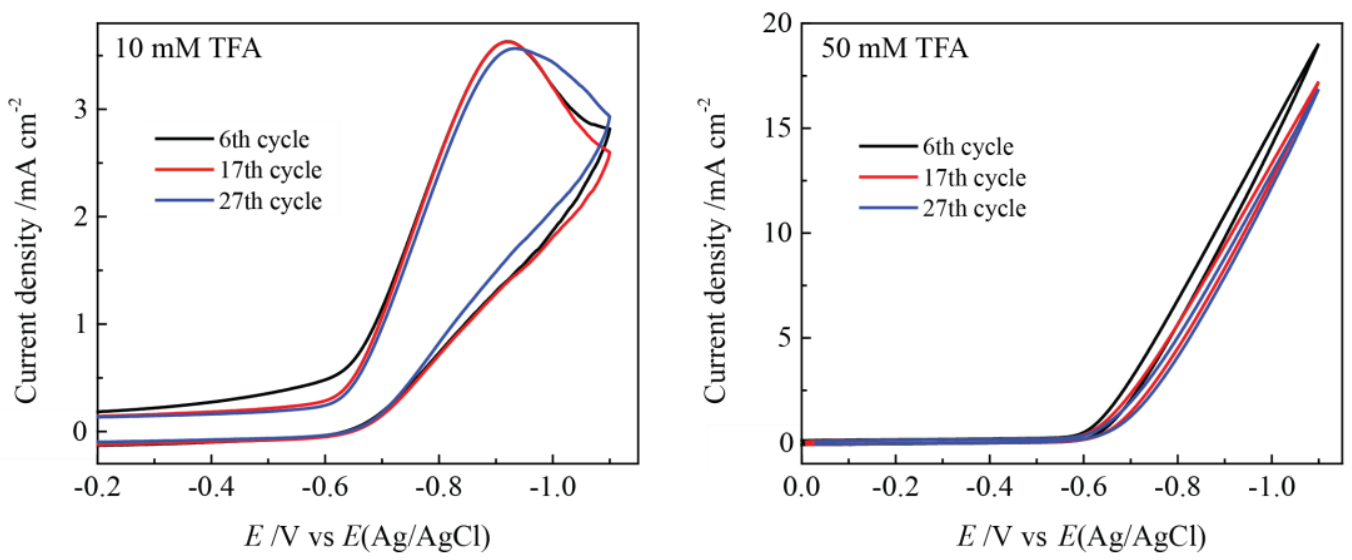

Figure 3.3 Repeated voltammetric cycling of an fd-RGO film that had been soaked in a $5 \mathrm{mM}$ solution of 1 in acetonitrile for $>1$ hr. Supporting electrolyte was deaerated $0.1 \mathrm{M} \mathrm{KPF}_{6}$ with 10 $\mathrm{mM}$ TFA (left) and $50 \mathrm{mM}$ TFA (right). The scan rate was $50 \mathrm{mV} / \mathrm{s}$.

Atomic Force Microscopy. To help explain the poor retention of $\mathbf{1}$ on RGO, graphene, and MWCNT in comparison to strong adsorption on fd-RGO and on graphite electrodes as reported earlier, ${ }^{217}$ AFM images of RGO, graphite, and fd-RGO were taken. These images as well as 
macroscopic photographs are shown in Figure 3.4. The images show no distinct differences in features between RGO and graphite; however, the calculated roughness of graphite (104 nm) is an order of magnitude larger than that of RGO (12 nm). The image of fd-RGO suggests a different morphology than either graphite or RGO. The surface appears to be a network or web of graphitic carbon. This can be seen more clearly by SEM (Figure 3.5). The roughness of fd-RGO (262 nm) is even higher than that of graphite.

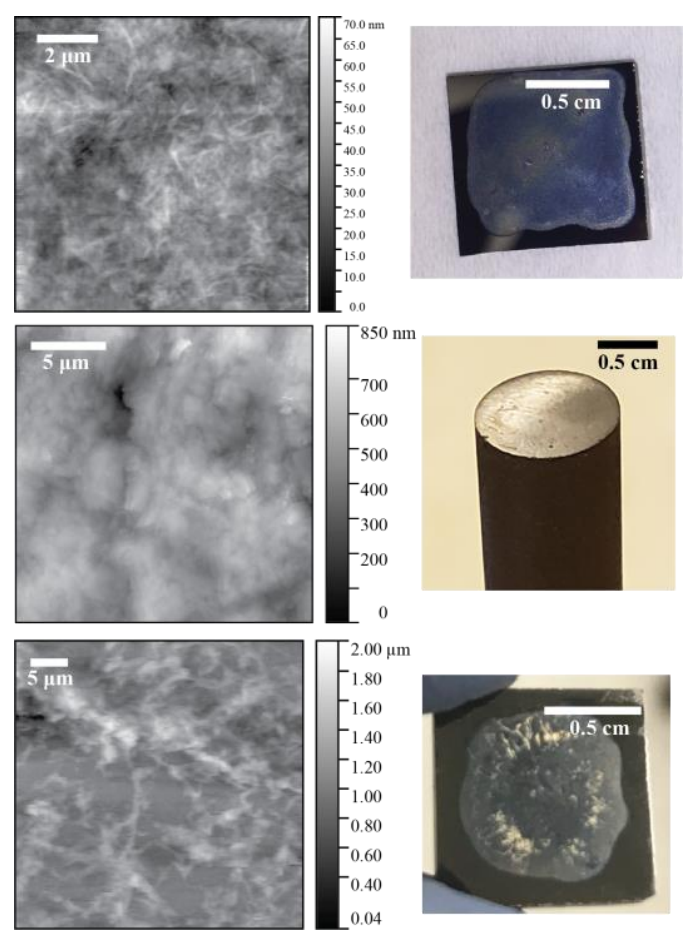

Figure 3.4 Atomic force micrographs (left) and optical photographs (right) of a dropcasted GO thin film (top), a polished graphite rod (middle), and a freeze-dried GO film (bottom). The RMS roughness values calculated for each of these materials are $12 \mathrm{~nm}$ for GO, $104 \mathrm{~nm}$ for graphite, and $262 \mathrm{~nm}$ for fd-RGO.

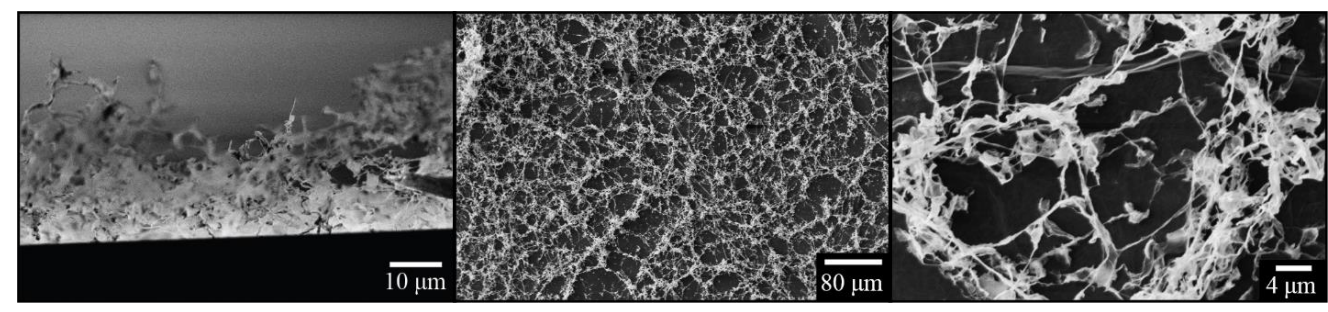

Figure 3.5 Scanning electron micrographs of a freeze-dried GO film on a silicon wafer. Cross section view is on the left. The middle and right images are top down views at different magnifications. 
AFM images were also taken of Nafion thin films in the hopes of elucidating why catalytic current decays so quickly even with a Nafion capping layer. These images can be seen in Figure 3.6. Nafion films on a degreased silicon wafer were smooth with very few features. There are occasional small circular indents in the film, a few hundreds of nanometers in diameter and less than $10 \mathrm{~nm}$ in depth. These could be attributed to holes in the film from nanoscopic bubbles formed in the solution as it dried. However, this changed dramatically when Nafion films were cast on carbon surfaces. On all three surface types, the Nafion film appear to have holes larger than $1 \mu \mathrm{m}$ in diameter and over $100 \mathrm{~nm}$ deep. These are the most pronounced on MWCNT and the least pronounced on graphene.
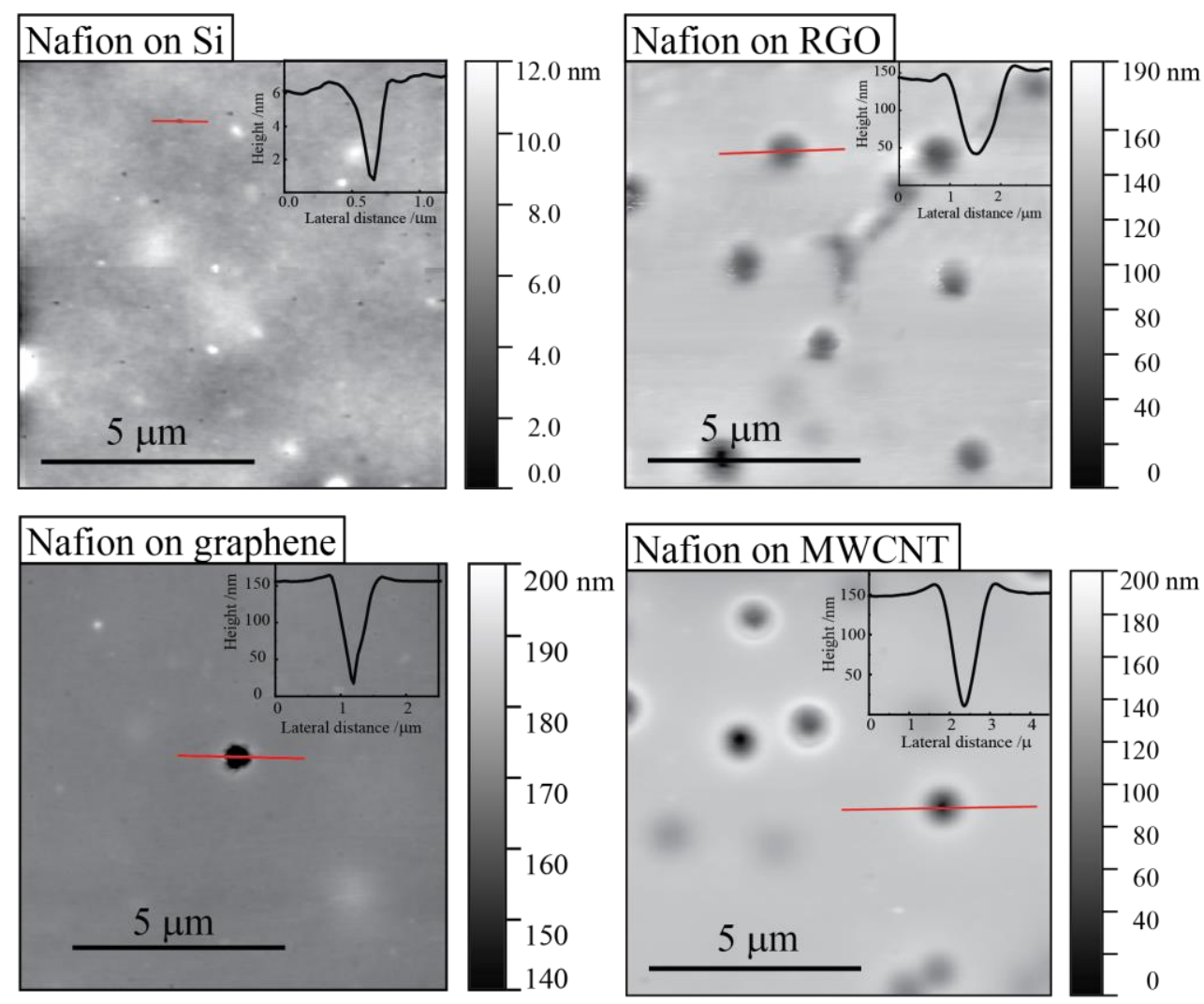

Figure 3.6 AFM images of Nafion thin films cast on a silicon wafer, an RGO film, single layer graphene, and a MWCNT film. The insets are the height profiles along the red line in each image. 


\subsection{Discussion}

It has previously been demonstrated by our laboratory that the proton reduction catalyst $\mathbf{1}$ adsorbs to graphite electrodes and remains adsorbed and catalytically active for several hours of electrolysis in solutions with a $\mathrm{pH}$ as low as $0.3 .^{69,217}$ The data in this chapter suggest that $\mathbf{1}$ can also be adsorbed to a variety of carbon thin films, namely reduced graphene oxide, single-layer graphene, and multi-walled carbon nanotubes. However, desorption from these surfaces under turnover conditions in acidic media is rapid. Correlation between surface roughness of these materials and stability of the catalytic current suggests that intercalation within the graphitic material plays a crucial role in retaining $\mathbf{1}$ during turnover.

Adsorption of 1 to carbon surfaces. XPS data in Figure 3.1 demonstrate that adsorption of 1 occurs on all three thin film materials, but the binding energy of the Co 2 p orbitals is not identical for each type of surface. The Co $2 \mathrm{p}$ signal of $\mathbf{1}$ on RGO appears at about $2.4 \mathrm{eV}$ higher in energy than on graphene and about $1.8 \mathrm{eV}$ higher than on MWCNT. In previously reported systems of coordination complexes adsorbed to graphene, a shift in binding energy of the metal center has been observed, but the shifts were to lower binding energy (compared to the free complexes). ${ }^{219-}$ 220 The large oxygen content of RGO compared to graphene and MWCNT may cause the shift to higher binding energy of the Co center in $\mathbf{1}$. The large oxygen content of RGO makes it more electron-withdrawing, effectively raising the binding energy of all orbitals on $\mathbf{1}$. This hypothesis should be confirmed with further experiments, however, such as XAS measurements. Additionally, the second peak at about $787 \mathrm{eV}$ in the Co $2 \mathrm{p}$ spectrum of RGO/1 suggests the presence of two different cobalt environments on RGO. To compare this spectrum with that in the literature, in reports by Eady et al., the spectrum of $\mathbf{1}$ on electrodeposited RGO also contains two cobalt peaks in the $2 \mathrm{p}$ region whereas the spectrum of $\mathbf{1}$ on graphite powder has only a single peak. ${ }^{217-218}$ It is unclear at this point what the additional cobalt peak on RGO substrates represents and additional experiments are required to determine its origin. However, one possible explanation is that the cobalt center of $\mathbf{1}$ can coordinate to one of the oxygen groups on RGO. This may also explain the large shift in binding energy of the main Co 2p peak. Further XPS experiments should be conducted using RGO at various stages of reduction to determine whether the oxygen functional groups on RGO influence either of the Co $2 p$ peaks (at $787 \mathrm{eV}$ and at $781.8 \mathrm{eV}$ ). These spectra can also be compared with the spectra of cobalt species in the literature and deconvoluted by fitting 
with multiple peaks to help determine what types of bonding environments or oxidation states of cobalt are present. Finally, it should be determined whether the presence of the additional cobalt environment on RGO influences the electrochemistry and electrocatalysis of $\mathbf{1}$.

Adherence of adsorbed 1 to surfaces under catalytic turnover. All four carbon thin films with 1 adsorbed show catalysis in acidic conditions (Figure 3.2). Unfortunately, however, in three of the cases the catalysis is not long-lived. Upon repeated cycling and addition of acid, the catalytic peak becomes less prominent and shifts to more negative potentials for RGO/1, graphene/1, and MWCNT/1. This is indicative of loss of catalyst from the surface. It is apparent from these data, then, that while 1 readily adsorbs to all of these surfaces initially, it becomes weakly adsorbed on RGO, graphene, and MWCNT during catalytic turnover or in acidic conditions. In the case of fd$\mathrm{RGO} / \mathbf{1}$, on the other hand, the catalytic current is stable over the course of the acid titration experiment. Furthermore, repeated cycling of fd-RGO/1 in $10 \mathrm{mM}$ TFA and $50 \mathrm{mM}$ TFA showed little change in the shape of the voltammograms, suggesting 1 does not readily desorb from this surface.

In the previously published graphite system, XPS coupled with sputtering experiments suggested that 1 was not only physisorbed to the surface of the graphite but also intercalated up to about $40 \mathrm{~nm}$ deep into the material. ${ }^{217}$ We therefore hypothesize that though initial adsorption of 1 to carbon surfaces occurs because of $\pi$ - $\pi$ interactions, these interactions alone are not strong enough to retain 1 during catalytic turnover. One possible explanation for this is that upon reduction, $\mathbf{1}$ becomes more negatively charged and this causes a repulsive force with the surface that competes with the $\pi-\pi$ attractive forces. Another possibility is that a contortion about the Co center may occur during catalysis and this would cause a weakening of the $\pi$ - $\pi$ interactions. This could be tested using EXAFS experiments. In any case, we hypothesize that intercalation within the graphite electrode dramatically improves retention of $\mathbf{1}$ under turnover conditions, and that intercalation is not possible on smooth RGO, graphene, and MWCNT thin films.

To support this hypothesis, AFM images were taken of a polished graphite rod (prepared in the same way as Reference 217), an RGO thin film, and a fd-RGO film. Chemically and structurally, graphite and RGO are nearly identical. Both are made up of overlapping sheets of partially oxidized aromatic carbon. However, from the AFM images in Figure 3.4, it is apparent that the microscopic morphologies of the surfaces are very different. The average roughness of the graphite surface is about an order of magnitude larger than that of RGO and the roughness of fd- 
RGO is about twice that of graphite. These data support the hypothesis described above because it suggests that the rougher graphite and fd-RGO surfaces are more porous and likely expose more edges of the graphitic sheets, allowing access for $\mathbf{1}$ to diffuse into the material. RGO thin films, on the other hand, display an extremely smooth surface, suggesting that the majority of the graphitic sheets lie flat on the surface, limiting the number of exposed edges and access sites for intercalation of 1. An exception may be the RGO published in Reference 218, on which catalyst adsorption was stable under turnover conditions in up to $2 \mathrm{mM}$ acid. This is a lower acid concentration than that used here, but this RGO was also likely rougher than the RGO used here as a result of the deposition method. The RGO in this thesis was dropcast from a colloidal suspension of GO onto smooth glassy carbon surfaces, whereas the RGO in Reference 218 was electrodeposited on an FTO electrode from a slurry of GO. Both the rougher FTO surface and the electrodeposition method are likely to produce rougher RGO films that are capable of intercalating $\mathbf{1 .}$

This effect can also explain why retention of $\mathbf{1}$ on graphene and MWCNT was minimal. Graphene is an atomically smooth surface and has no layers that could allow intercalation. MWCNT films are rougher than graphene; however, these films do not have the stacked sheets structure that graphite and RGO have. Instead a MWCNT film is made up of a web of smooth tubes, which may not facilitate trapping of $\mathbf{1}$.

Further experiments to confirm the hypothesis that intercalation plays a role in catalyst retention should be conducted. XPS experiments coupled with ion sputtering can identify whether 1 intercalates within RGO films. These experiments have previously been conducted on graphite electrodes and have shown that $\mathbf{1}$ does intercalate several tens of nanometers into the material. ${ }^{217}$

As an attempt to improve retention of $\mathbf{1}$ on the smooth carbon films where intercalation may not be possible, Nafion films were cast onto the surfaces after $\mathbf{1}$ had been adsorbed. Nafion is a proton-conducting polymer and should allow protons from the bulk solution access to $\mathbf{1}$ at the electrode surface while preventing 1 from diffusing away from the electrode into solution. Unfortunately, however, the retention of $\mathbf{1}$ on all carbon surfaces with the addition of Nafion is not significantly different than without Nafion. In all cases catalytic current begins to drop off around $8 \mathrm{mM}$ acid concentration (Figure 3.2). In the case of graphene/1, however, the Nafion appears to keep the catalyst on the surface for longer than without Nafion. On a bare graphene/1 surface, the current in $4 \mathrm{mM}$ acid is only marginally higher than in $2 \mathrm{mM}$ acid, whereas with Nafion, catalytic current appears to increase with addition of acid until the acid concentration is $8 \mathrm{mM}$. It is worth 
noting that when a Nafion film is present on any of the three electrode surface types, the onset of current occurs at more positive potentials compared to electrodes without Nafion. The reason for this is still unknown but hypothesized to be due to a different local $\mathrm{pH}$ at catalytic sites in a Nafion matrix because of the acidic nature of Nafion itself. A poly-2-vinylpyridine (an electron conducting polymer) thin film cast on each of these surfaces demonstrated a similarly poor ability to prevent 1 from desorbing.

In order to understand the weak retention of 1 with a Nafion capping layer, AFM images of Nafion films are shown in Figure 3.6. On all three carbon thin films, the Nafion layer contains large, circular holes, up to $2 \mu \mathrm{m}$ in diameter and $150 \mathrm{~nm}$ deep. Because of the highly symmetric shape of the holes, they are attributed to air bubble formation in the Nafion solution as it dries into a film. Because graphitic surfaces are generally more hydrophobic than silicon surfaces that have been etched with $\mathrm{O}_{2}$ plasma, we predict that the alcohol/water solution of Nafion wets graphitic materials more poorly and therefore is more likely to trap large air bubbles than when dropcast onto a plasma-etched silicon surface.

\subsection{Conclusions}

In this work a molecular proton reduction electrocatalyst (1) was immobilized on graphitic carbon electrodes through $\pi-\pi$ interactions. The graphitic surfaces studied here are reduced graphene oxide (RGO) thin films, single-layer graphene, and multiwalled carbon nanotube (MWCNT) thin films. This work complements previous reports on the adsorption of $\mathbf{1}$ on graphite rod electrodes and RGO thin films on FTO-coated glass electrodes. ${ }^{69,217-218}$ X-ray photoelectron spectroscopy demonstrates that $\mathbf{1}$ spontaneously adsorbs to the three types of graphitic surfaces, but with a difference in binding energy. In particular, two different cobalt binding energies are observed on RGO, which is consistent with previous reports. ${ }^{218}$ Further experiments are necessary to understand this phenomenon. Electrochemical data show fast desorption from the surfaces under turnover conditions, but retention of the catalyst under turnover is dramatically improved when a rough RGO film is used (freeze-dried RGO) instead of a smooth dropcasted film. Based on these data and the previous reports of long-term retention on graphite electrodes, we hypothesize that intercalation within the graphitic structure plays a crucial role in immobilizing $\mathbf{1}$ on these carbon materials.

Physisorption on carbon surfaces is a facile, cost-effective method for immobilizing molecular catalysts on electrode surfaces. In particular, the catalyst in this report requires no 
synthetic modifications in order to physisorb through $\pi$-stacking on a graphitic surface; and graphitic thin films, particularly RGO, are easily and cheaply fabricated. This system demonstrates a cost-effective approach toward heterogenizing molecular electrocatalysts. Future work should be directed toward fabricating transparent RGO thin films with sufficient roughness to retain adsorbed $\mathbf{1}$ during electrolysis. This might be achieved, for example, by dropcasting GO on a preroughened surface or by roughening the GO film after dropcasting (e.g. with a laser-cutter).

Future experiments. First, as mentioned above, the nature of the catalyst adsorbed to each graphitic surface should be identified. The chemical state of the catalyst can be determined using $\mathrm{x}$-ray photoelectron and $\mathrm{x}$-ray absorption spectroscopies. One hypothesis described in the previous section states that $\mathbf{1}$ may coordinate to oxygen functional groups on partially oxidized graphitic surfaces (i.e. RGO and polished graphite) in addition to $\pi$-stacking with the graphitic network. This hypothesis can be supported by adsorbing 1 to RGO containing varying quantities of oxygen functional groups and monitoring the binding energy in XPS and the coordination number and structure by XANES and EXAFS. Furthermore, it can be shown whether 1 intercalates within these graphitic materials by using XPS coupled with sputtering experiments.

Next, the stabilities of the systems described in this chapter should be quantified. Specifically, the rate of desorption of the catalyst from each type of surface should be quantified in different $\mathrm{pH}$ levels. This can be done by performing bulk electrolysis coupled with XPS measurements. For example, electrodes with adsorbed $\mathbf{1}$ should be held at a specific potential in a solution with a defined $\mathrm{pH}$ for various lengths of time. XPS before and after the electrolysis experiment can determine how much catalyst has been lost from the surface. In XP spectra of these surfaces, the total amount of catalyst cannot be quantified, but by taking the ratio of Co $2 p$ to $C 1 s$ intensity, the relative amount of catalyst can be determined. 


\section{Chapter 4. Reduced Graphene Oxide Thin Films Embedded with a Molecular Catalyst: Kinetic Considerations}

Paper in preparation.

\subsection{Introduction}

Previous studies have investigated kinetic parameters of electrocatalytic thin films that undergo simple outer-sphere, one-electron transfers. ${ }^{221-224}$ Most of these studies include electrocatalytic polymers, such as the polyvinylpyridine electrode coatings that incorporate $\mathrm{IrCl}_{6}{ }^{2-}$ and $\mathrm{Ru}^{\mathrm{II}}(\mathrm{edta})$ complexes described by Oyama and $\mathrm{Anson}^{225}$ or the poly[Ru(vbpy)$\left.)_{3}\right]^{2+}$ films described by Ikeda, et $a .^{226}$ among others. ${ }^{227-230}$ These systems undergo one-electron transfers with metal complexes in solution. Through this work, methods have been developed for determining rate limiting factors in electrocatalytic thin films, and numerical values for parameters such as substrate diffusion and electron diffusion within a film were derived.

In addition to these, many reports have described the kinetics of more complex electrocatalytic reactions. These include the oxidation of formaldehyde at a porphyrin-based polymer on graphite, ${ }^{231}$ reduction of $\mathrm{CO}_{2}$ using a surface-bound Re polymer, ${ }^{232}$ reduction of $\mathrm{O}_{2}$ at Pt nanoparticles dispersed in a polyaniline film, ${ }^{233}$ reduction of $\mathrm{CO}_{2}$ using cobalt sites coordinated in a polymer on graphite electrodes, ${ }^{234-235}$ and others. ${ }^{236-238}$ Fewer reports, however, have investigated the kinetics of electrocatalytic proton reduction in electrocatalytic films. ${ }^{239-242}$ This area warrants further study as the proton reduction half reaction is relevant in electrochemical energy conversion and storage. Understanding electrocatalytic generation of hydrogen in thin films on electrode surfaces is crucial in developing efficient water splitting systems.

Reduced graphene oxide (RGO) thin films, as described in Chapter 2, are an ideal platform in which to load electrocatalysts. RGO is made up of small sheets (nanometer to microns in diameter) of partially oxidized graphene. Because RGO is fabricated from a solution-processable precursor (graphene oxide), it can be cast as a thin film with controllable thickness. Furthermore, RGO thin films can easily be loaded with an electrocatalyst by simple adsorption, as shown in 
Chapter 3. While RGO has been commonly used as a support for electrocatalysts, ${ }^{217-218,}$, 243-246 studies on the fundamental kinetics of electrocatalyst-embedded RGO films are extremely rare. One relevant example, by Huang and coworkers, determined kinetic parameters through cyclic voltammetry and electrochemical impedance spectroscopy of a film formed by alternating RGO sheets and layers of a cobalt porphyrin proton reduction electrocatalyst. ${ }^{247}$ This study, however, assumed the electrocatalytic reaction itself was rate-limiting. This chapter takes an alternative viewpoint: limitations on electrocatalytic current by diffusion and/or electron transport within the film cannot be ruled out a priori. Because the structure of RGO films is significantly different than that of polymer or metal oxide films, rates of electron transport and substrate diffusion within the film are likely also different. It is therefore necessary to identify and understand rate limiting behaviors in electrocatalytic RGO films.

Herein we report kinetic details of proton reduction by the molecular catalyst $\mathbf{1}$ (highlighted in Chapter 3) when embedded within an RGO thin film. Catalyst 1 was chosen because of its high catalytic activity for proton reduction, and because its ability to $\pi$-stack on graphitic surfaces should decrease the likelihood of diffusing out of the RGO film. ${ }^{69}$, 217-218, 248 Graphene oxide films embedded with 1 were dropcasted on glassy carbon electrodes and reduced electrochemically to RGO. Thickness of the films and catalyst density within the films were controlled by manipulating the volume and concentration of the GO-catalyst casting solution.

Figure 4.1 describes the processes that occur within these thin films during electrocatalysis. In these RGO films, cobalt catalyst is distributed throughout the film and is symbolized as $\mathrm{Co}(\mathrm{III})$ in Figure 4.1, as this is the cobalt oxidation state of $\mathbf{1}$ under ambient conditions. To initiate catalysis, the cobalt center is reduced by two electrons to Co(I). This form of the catalyst then converts protons within the film to hydrogen molecules. The rate constant of this reaction $(k)$, the diffusion of protons into the film from the bulk solution $\left(\mathrm{DH}^{+}\right)$, and the diffusion of hydrogen molecules out of the film to the bulk solution $\left(\mathrm{DH}_{2}\right)$ each may be a kinetic limit on the obtainable current. Additionally, the movement of electrons, here defined as the diffusion of electrons through the film $\left(D_{e}\right)$, may also limit the current.

The intent of the study was to identify operating conditions that maximize the electrocatalytic current and turnover frequency by identifying which of the processes in Figure 4.1 primarily limit the current. Based on cyclic voltammetry measurements, it was determined that below a certain thickness, current is primarily limited by slow diffusion of hydrogen out of the 
film. Above this thickness, the current is limited by a convolution of hydrogen diffusion and electronic resistivity of the RGO. An optimal film thickness was determined to be between 200 and $500 \mathrm{~nm}$. Future experiments are delineated that will identify the optimal catalyst density.

electrode

film

solution

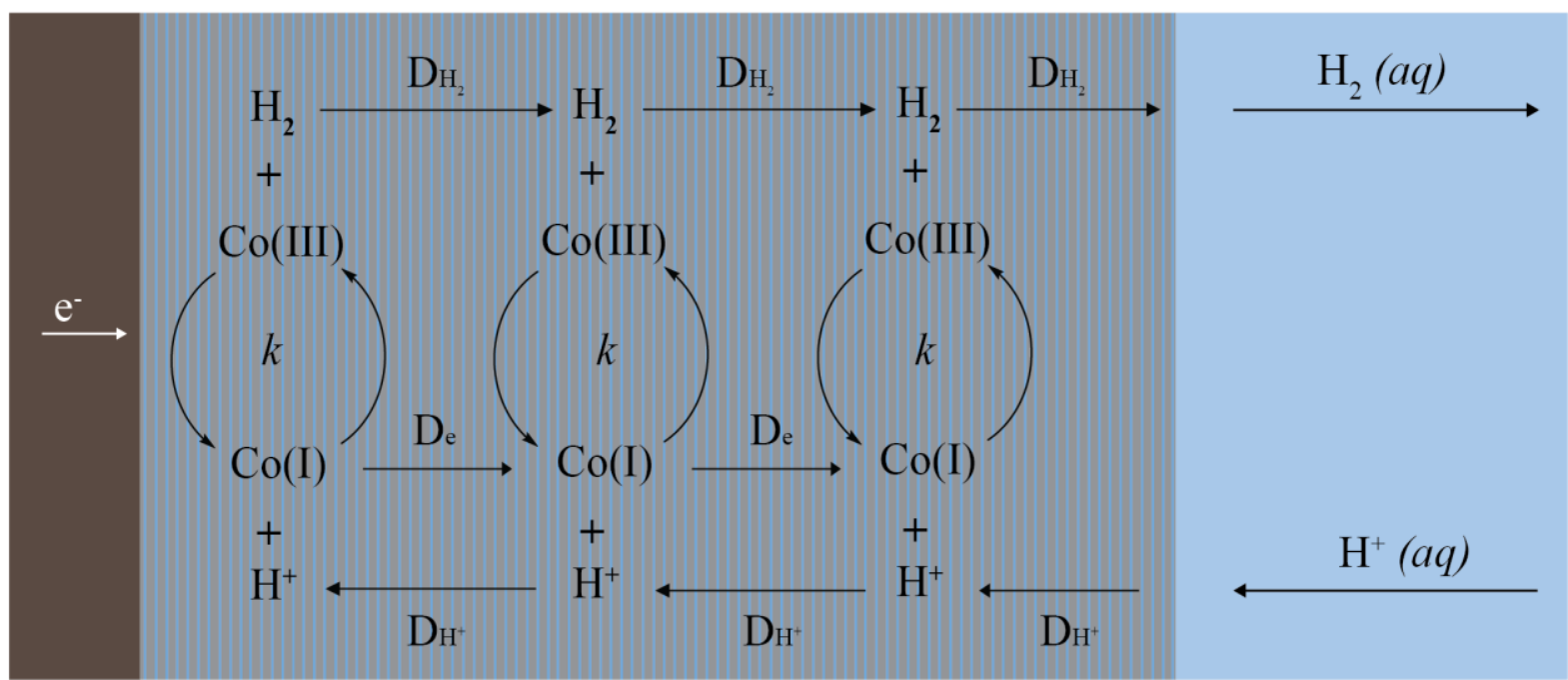

Figure 4.1 Diagram showing the processes that occur within a thin film on an electrode surface during electrocatalysis. The symbols are as follows: $k$ is the rate constant for the catalytic reaction; $\mathrm{DH}^{+}$is the diffusion coefficient of protons within the film; $\mathrm{DH}_{2}$ is the diffusion coefficient of hydrogen within the film; $D_{e}$ is the diffusion coefficient of electrons within the film; $\mathrm{Co}$ (III) is oxidized form of the catalyst; $\mathrm{Co}$ (II) is the reduced form of the catalyst after one electron transfer.

\subsection{Experimental}

Materials. Sodium phosphate dibasic, phosphoric acid, trifluoroacetic acid (TFA, 99\%, Sigma Aldrich), potassium permanganate (98\%, Acros), sulfuric acid (ACS grade, Fisher Scientific Education), and hydrogen peroxide ( $30 \%$ in $\mathrm{H}_{2} \mathrm{O}$, Sigma Aldrich) were used as received. Potassium hexafluorophosphate $(95 \%, \mathrm{TCI})$ was recrystallized from aqueous $0.1 \mathrm{M} \mathrm{KOH}$ before use. Graphite flakes (-20+84 mesh, Johnson Matthey) were used as the carbon source for graphene oxide suspensions. Water with a resistivity $>18.2 \mathrm{M} \Omega$ (Nanopure Barnstead Water Purification) was used throughout. Glassy carbon electrodes ( $3 \mathrm{~mm}$ diameter disk electrodes, $\mathrm{CH}$ Instruments) were prepared by polishing with alumina ( $1 \mu \mathrm{m}, 0.3 \mu \mathrm{m}$, and $0.05 \mu \mathrm{m}$ alumina suspensions) on felt 
and rinsing with water. Polished $\operatorname{Si}(111)$ wafers from Wafer Works doped with As $\left(\leq 0.006 \Omega \mathrm{cm}^{-}\right.$ ${ }^{1}$ ) were used as substrates for AFM imaging.

Preparation of graphene oxide suspensions. See Chapter 2.2 Experimental for details on preparing graphene oxide.

Synthesis of 1. In an inert atmosphere box under $\mathrm{N}_{2}$ pressure (Innovative Technologies), a solution of 3,6-dichloro-1,2-benzenedithiol (0.5 g, $2.4 \mathrm{mmol})$ and sodium methoxide (0.128 g, 2.4 mmol) was added dropwise to a suspension of cobalt(II) sulfate heptahydrate $(0.320 \mathrm{~g}, 1.14 \mathrm{mmol})$ in $40 \mathrm{~mL}$ of dry methanol in a roundbottom flask. The addition caused a color change to deep blue. The resultant solution was stirred for 2 hours. A solution of tetrabutylammonium bromide $(0.407$ $\mathrm{g}, 1.2 \mathrm{mmol}$ ) in $6 \mathrm{~mL}$ dry methanol was added at this time, and the solution was stirred for an additional 2 hours. The sealed flask was then removed from the glovebox and the solvent volume was reduced under vacuum to $<10 \mathrm{~mL}$, resulting in a thick, dark blue slurry. This was filtered and dried. The solid was then recrystallized from dichloromethane/ether to yield $\mathbf{1}$ as a dark blue crystalline solid (50\% yield). The UV-visible absorption spectrum matched that in the literature. ${ }^{218}$

Preparation of $R G O$ and catalyst-embedded RGO thin films. A small volume of the graphene oxide suspension was removed and sonicated for 20 minutes to exfoliate and disperse fully individual graphene oxide sheets. This volume was then centrifuged at $9000 \mathrm{rpm}$ for 30 minutes. The brown supernatant was removed. Its concentration was approximately $2 \mathrm{mg} \mathrm{mL}^{-1}$. This suspension was combined with a defined volume of a solution of $\mathbf{1}$ in acetonitrile or with the same volume of clean acetonitrile and then dropcasted on a glassy carbon surface and allowed to air dry. The thickness of the film was controlled by controlling both the volume and the concentration of the dropcasted suspension. The films were reduced to RGO electrochemically by cycling between open circuit potential and $-1 \mathrm{~V}$ ten times in the same $\mathrm{pH} 2$ electrolyte solution used for electrochemical measurements.

Electrochemical measurements. Electrochemical measurements were taken using a $\mathrm{CH}$ Instruments $760 \mathrm{C}$ potentiostat. A three-electrode cell was used with a graphite rod as the counter electrode and $\mathrm{Ag} \mid \mathrm{AgCl}$ (sat'd $\mathrm{KCl}$ ) (in a compartment separated by a glass frit) as the reference electrode. All potentials are referenced to $\mathrm{Ag} \mid \mathrm{AgCl}$ (sat'd $\mathrm{KCl}$ ). For measurements taken in a nonbuffered solution, the electrolyte was deaerated $0.1 \mathrm{M} \mathrm{KPF}_{6}$ with trifluoroacetic acid ( $\mathrm{pH} 2$ ). For measurements taken in a buffered solution a deaerated $\mathrm{pH} 2$ phosphate buffer of approximately 0.2 M concentration (prepared from sodium phosphate dibasic and phosphoric acid) was used. All 
voltammetry presented in this chapter was background subtracted by averaging four voltammograms of RGO films containing no catalyst and subtracting this averaged data from the voltammetry of the RGO film with catalyst of the same thickness. The data points in the current and TOF versus thickness plots are averages of four background-subtracted voltammograms.

Atomic Force Microscopy. Film thickness was measured by dropcasting a 3-mm diameter droplet of the appropriate volume and concentration of graphene oxide on a degreased polished silicon wafer. After drying, a clean syringe needle was used to gently score the film in several places to create a step edge. A Veemco Dimension Icon Atomic Force Microscope in contact mode was used to collect images. A sample image is shown in Figure 4.2. The thickness was estimated by measuring the height along the dotted blue line and averaging vertically between the two dotted white lines. At least three regions on between one and three different samples for each film thickness were measured and averaged. The error bars for these thicknesses are the standard deviation of these averages.

Optical Profilometry. An Olympus LEXT OLS4100 laser scanning digital microscope was used to measure the thickness of the thickest film. One region on a single film was measured and averaged over $50 \mu \mathrm{m}$. The error bar for this data point is based on the precision of the instrument $(10 \mathrm{~nm})$ rather than the average of discrete samples (in contrast to the data points based on AFM measurements).
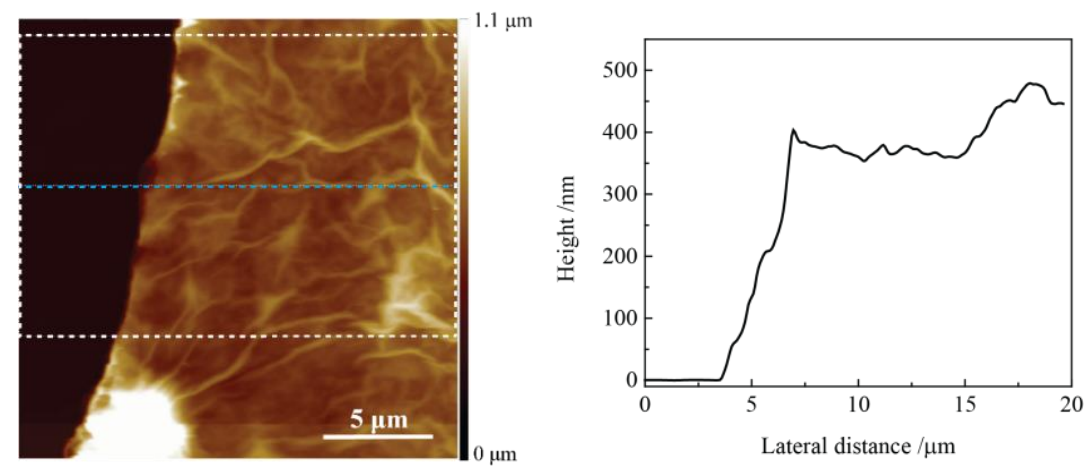

Figure 4.2 (Left) Representative AFM image of an edge site on a $200 \pm 100 \mathrm{~nm}$ film. The film thickness was measured in this region by taking a profile across the blue dashed line and averaging vertically between the two horizontal white dashed lines. This averaged profile is shown on the right. 


\subsection{Results}

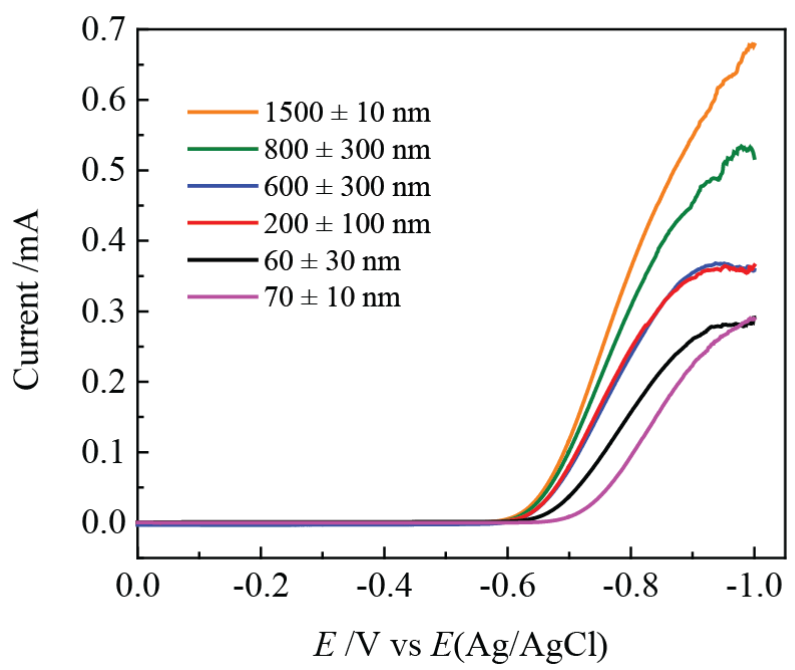

Figure 4.3 Background subtracted linear sweep voltammetry of a series of film thicknesses with a catalyst density of $2 \times 10^{-4}$ mol catalyst per $\mathrm{cm}^{3}$ of RGO. The supporting electrolyte was a deaerated $\sim 0.2 \mathrm{M}$ phosphate buffer at $\mathrm{pH} 2$. Scan rate was $10 \mathrm{mV} \mathrm{s}^{-1}$.

Figure 4.3 shows background-subtracted linear sweep voltammograms of a series of $\mathrm{RGO} / \mathbf{1}$ films of various thicknesses in a $\mathrm{pH} 2$ buffer. The volumetric density of $\mathbf{1}$ within these films was kept constant (about $2 \times 10^{-4} \mathrm{~mol}$ catalyst per $\mathrm{cm}^{3}$ of RGO), meaning thicker films contain more catalyst than thinner films. The voltammogram for each film begins to rise just negative of $0.6 \mathrm{~V}$. For the thinner films, the current appears to reach a plateau at negative potentials, around $0.9 \mathrm{~V}$. The thicker films do not reach a plateau within this potential window. At a given potential within this window, the current for each film increases with increasing film thickness (i.e. increasing total loading of $\mathbf{1}$ ). The current and turnover frequencies from these voltammograms are plotted versus film thickness in Figure 4.4. Figure 4.4a plots the current at the relatively low potential of $-0.95 \mathrm{~V}$, where current is high. Unfortunately, because of the low quantities of catalyst within the films, the $E_{1 / 2}$ of $\mathbf{1}$ was not determined. Previous studies on this catalyst in solution ${ }^{248}$ and adsorbed to graphite electrodes ${ }^{217}$ report the $E_{1 / 2}$ as -0.46 and $-0.65 \mathrm{~V}$, respectively, vs $\mathrm{Ag} \mid \mathrm{AgCl}$ at $\mathrm{pH}$ 7. The data in Figures 4.4a and $4.4 \mathrm{~b}$ were taken at $-300 \mathrm{mV}$ overpotential with respect to the $E_{1 / 2}$ of the catalyst on a graphite electrode. At this potential, the current continually rises as thickness increases. If a point at the origin is considered in addition to the data shown (at $0 \mathrm{~nm}$ film thickness, the current should be $0 \mathrm{~mA}$ ) then the current appears to steeply increase at 
lower film thicknesses and begins to reach a plateau with thicker films. This trend is consistent with what has been seen in the literature. For example, simulated cyclic voltammograms by $\mathrm{C}$. Costentin and J.-M. Saveant under conditions where the solvent is the substrate for electrocatalysis showed plateaued currents as film thickness increased. ${ }^{222}$ It should be noted that the data point corresponding to a $600 \mathrm{~nm}$ film does not follow a clear trend with the other data points and should probably be re-measured. In Figure $4.4 \mathrm{~b}$, the turnover frequency (TOF) calculated from the voltammograms at $-0.95 \mathrm{~V}$ is plotted. TOF values at this potential decrease as film thickness increases, leveling off around $7 \mathrm{~s}^{-1}$.

Figure $4.4 \mathrm{c}$ plots the current at a potential closer to the onset of catalysis, $-0.67 \mathrm{~V}$, approximately $20 \mathrm{mV}$ negative of the reported $E_{1 / 2}$ for $\mathbf{1}$ on graphite electrodes at $\mathrm{pH}$ 7. At this potential current is very low, so the effects of product $\left(\mathrm{H}_{2}\right)$ buildup within the film should be minimal. The shape of the current versus thickness curve is similar to that at $-0.95 \mathrm{~V}$. Interestingly, however, the shape of the TOF versus thickness curve (Figure 4.3d) is quite different. TOF increases with increasing film thickness, reaching a maximum around $200 \pm 100 \mathrm{~nm}$ and then decreasing.

To further determine limiting factors on current, a series of films of varied thickness with a constant amount of catalyst $\left(1.7 \pm 0.2 \times 10^{-10}\right.$ moles $)$ were prepared. Figure 4.5 shows a set of
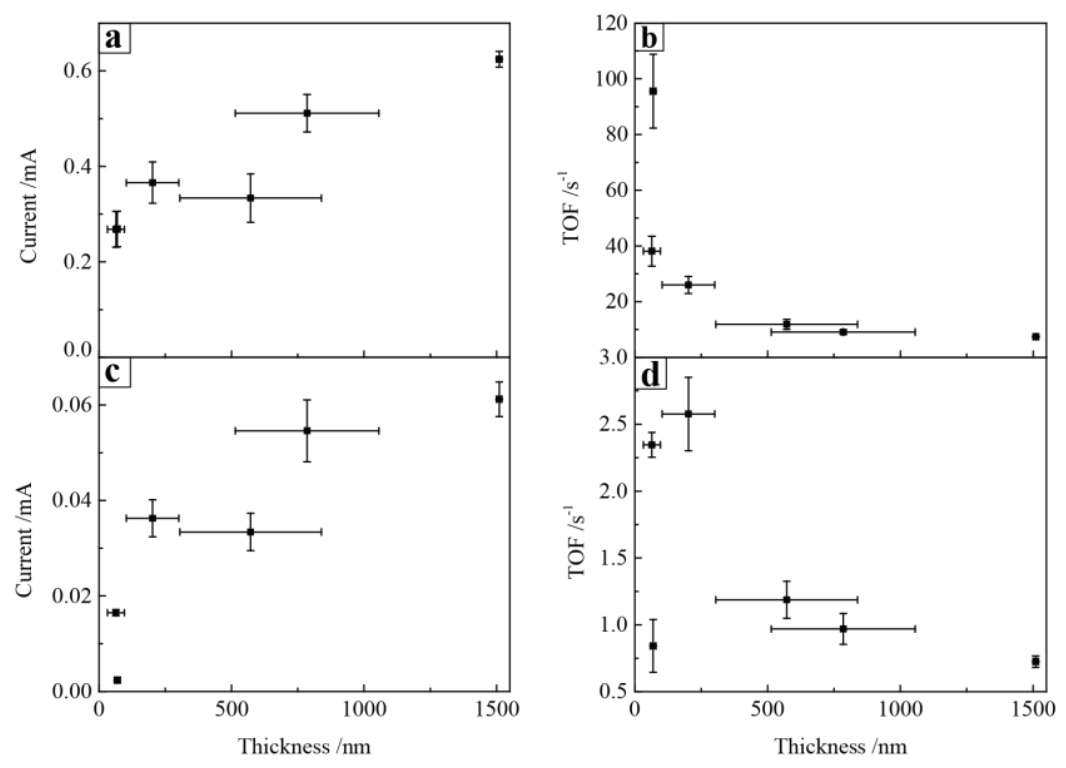

Figure 4.4 Plotted current and TOF versus film thickness from background-subtracted voltammetry in Figure 4.3. (a) Current at -0.95 V. (b) TOF at -0.95 V. (c) Current at -0.67 V. (d) TOF at $-0.67 \mathrm{~V}$. The data point corresponding to a $600 \mathrm{~nm}$ film does not follow a clear trend with the other data points and should probably be re-measured. 


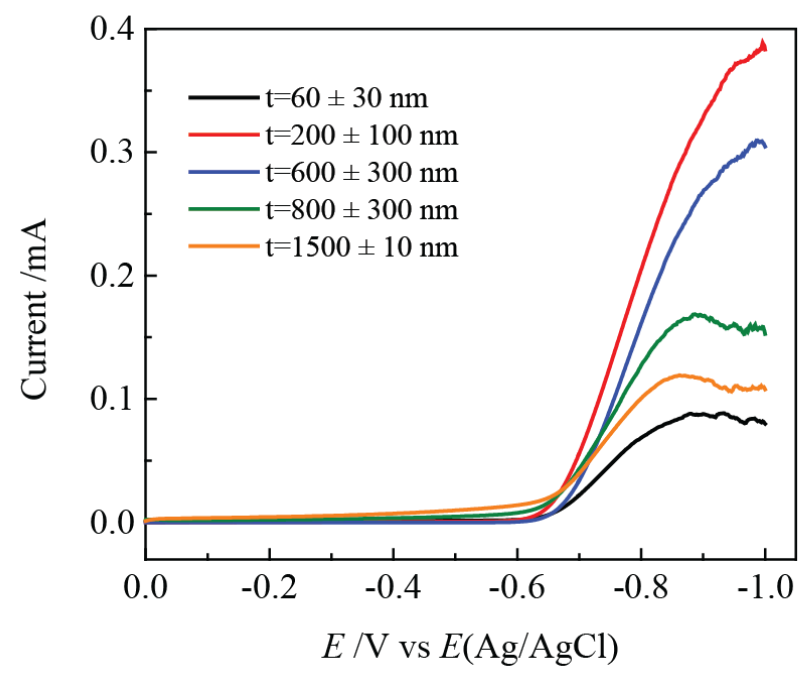

Figure 4.5 Background subtracted linear sweep voltammetry of a series of film thicknesses with $1.7 \pm 0.2 \times 10^{-10}$ mole catalyst in each film. The supporting electrolyte was a deaerated $\sim 0.2 \mathrm{M}$ phosphate buffer at $\mathrm{pH}$ 2. Scan rate was $10 \mathrm{mV} \mathrm{s}^{-1}$.
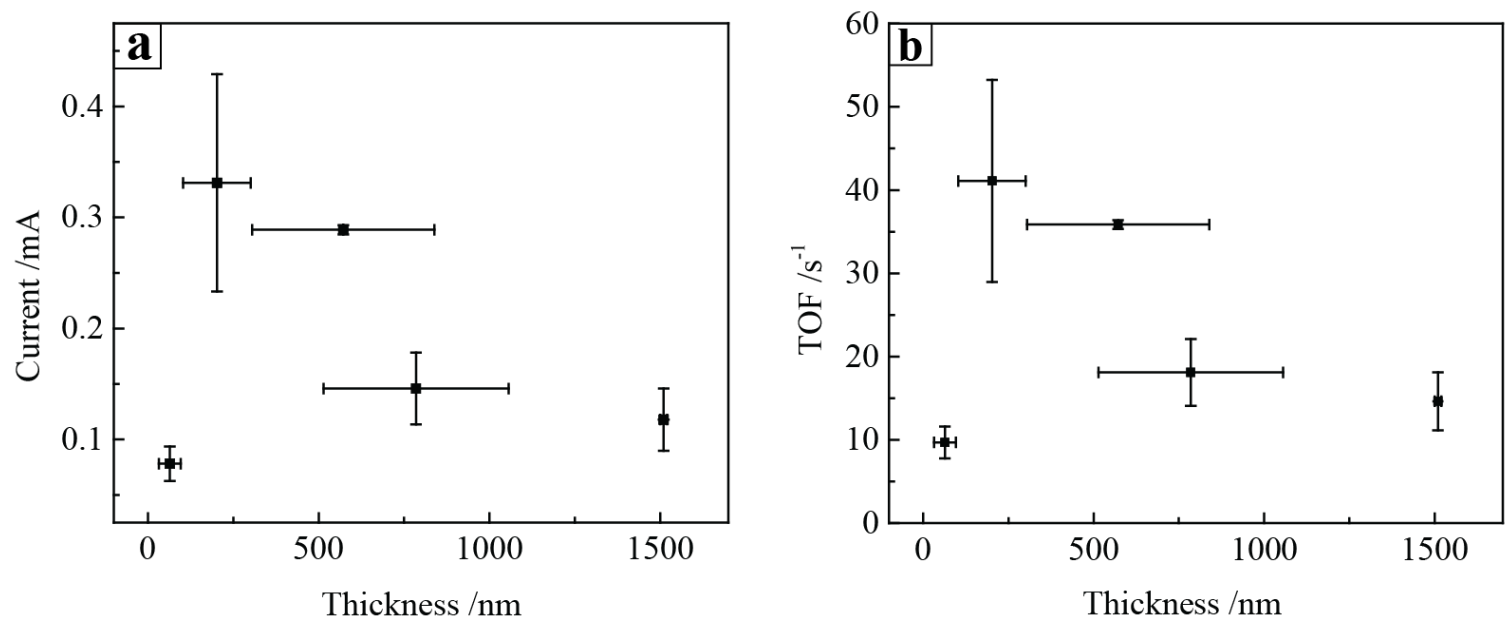

Figure 4.6 Plotted current (a) and TOF (b) versus film thickness from the background-subtracted voltammetry in Figure 4.5.

voltammograms from this series of films. It is obvious that the same trend as Figure 4.3 is not seen here - the current does not trend upward with film thickness. Figure 4.6 plots the current at -0.95 $\mathrm{V}$ versus film thickness and Figure $4.6 \mathrm{~b}$ plots the TOF at this potential. Current and TOF increase 
with increasing film thickness up to between 200 and $600 \mathrm{~nm}$ where it then begins to drop off. Interestingly, this is a similar trend that is seen with TOF in Figure 4.4d.

Figure 4.7 shows cyclic voltammograms of two electrodes containing layered films. One electrode was prepared by dropcasting a $600 \pm 300 \mathrm{~nm}$ film containing $1.7 \pm 0.2 \times 10^{-10}$ moles of catalyst, and after drying, a second $600 \pm 300 \mathrm{~nm}$ film with no catalyst was cast on top. The voltammogram for this electrode is in black. The other electrode was prepared in a reverse order: a $600 \pm 300 \mathrm{~nm}$ film with no catalyst was first cast and dried and then a $600 \pm 300 \mathrm{~nm}$ film containing $1.7 \pm 0.2 \times 10^{-10}$ moles of catalyst was cast on top. The voltammogram for this electrode is in red. Both voltammograms reach similar currents. In comparison, in blue is the voltammogram of the $600 \pm 300 \mathrm{~nm}$ film with $1.7 \times 10^{-10}$ moles of catalyst taken from Figure 4.5. This film reaches a much higher current than either of the layered films.

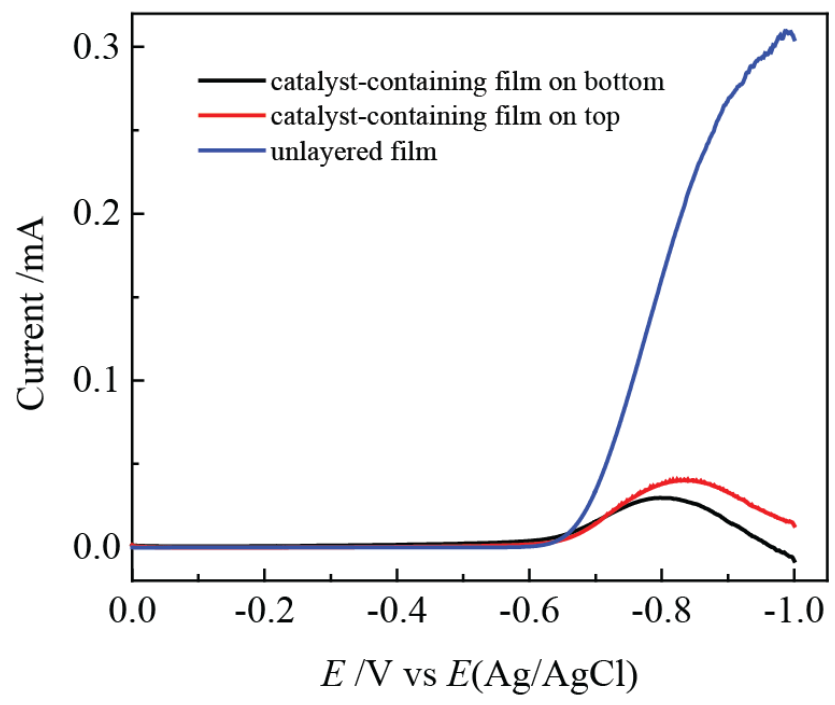

Figure 4.7 Background subtracted linear sweep voltammetry of three catalyst containing films. In blue is the voltammogram from Figure 4.5 (the $600 \pm 300 \mathrm{~nm}$ film containing $1.7 \pm 0.2 \times 10^{-10} \mathrm{~mol}$ catalyst). In black and red are voltammograms from similar films but with an additional $600 \pm 300$ $\mathrm{nm}$ RGO film cast on top of and below, respectively, the catalyst-containing film. Supporting electrolyte was a deaerated $\sim 0.2 \mathrm{M}$ phosphate buffer at $\mathrm{pH}$. Scan rate was $10 \mathrm{mV} \mathrm{s}^{-1}$.

\subsection{Discussion}

From the data described above we conclude that electrocatalytic proton reduction in RGO films embedded with the catalyst $\mathbf{1}$ is limited by both product buildup within the film (i.e. $\mathrm{H}_{2}$ 
diffusion) and electronic conductivity of the films. For thinner films (below about $500 \mathrm{~nm}$ ), hydrogen diffusion out of the film is the limiting factor, and for films thicker than about $500 \mathrm{~nm}$, current is limited by a convolution of both hydrogen diffusion and electronic conductivity of the films.

The data in Figures $4.4 \mathrm{a}$ and $4.4 \mathrm{~b}$ demonstrate two things. First, both substrate and electrons can penetrate the film (i.e. more than just the surface layer of catalyst is active). This is evidenced by the increase in current with thicker films. Second, film thickness plays a role in limiting the rate of catalysis. This is apparent because of the plateau that the current reaches with thicker films and because of the decrease in TOF as film thickness increases. Therefore, current is likely not limited by the electrocatalytic rate constant and is instead limited by one of three things related to film thickness: proton diffusion into the film, electronic conductivity of the film, or hydrogen diffusion out of the film.

C. Costentin and J.-M. Savéant published three papers on the use of cyclic voltammetry to analyze kinetics of electrocatalytic thin films. ${ }^{222-224}$ In these reports voltammetry is simulated under several assumptions. One, the catalytic reaction is an outer-sphere, one-electron transfer between a catalytic site within the film and a dissolved species than can penetrate the film. Two, the catalyst is homogeneous throughout the film, meaning every catalyst site operates by the same mechanism and at the same electrochemical potential and there is a uniform distribution of catalyst throughout the film. Three, the electrode surface is not active for electrocatalysis in the reported potential range (in other words, the only redox chemistry that occurs is electrocatalysis at catalytic sites and self-exchange between adjacent catalyst sites). Four, electron conduction within the film operates by a hopping mechanism and can therefore be described by Fick's laws of diffusion. The authors also assume that only three factors can affect electrocatalytic current: substrate diffusion, electron diffusion, and reaction rate. In each paper, one of these factors is presumed to be fast, thereby reducing the number of possible limiting factors. In the following paragraphs I compare the data in Figure 4.4 to the models in these three papers and show that the predictions from the models do not match the data.

In each of these reports, current is plotted versus film thickness and the trend in Figure 4.4 can be observed, namely an increase in current with film thickness eventually reaching a plateau. This trend is shown in Figure 4.8, and the similarity between this figure and Figures 4.4a and c are apparent. According to these reports, the intersection of the linear portion of the current vs 
thickness curve with the plateau current is defined as the optimum film thickness, $d_{f}^{o p t}$. In the case where the catalytic reaction is fast ( $k$ is large), $d_{f}^{o p t}$ is defined by equations 4.1 and $4.2 .{ }^{224}$ Equation 4.1 applies when electron diffusion is far smaller than substrate diffusion. Equation 4.2 applies when substrate diffusion is far smaller than electron diffusion. The parameters in these equations are as follows: $d_{f}^{o p t}$ is the optimum film thickness; $D_{e}$ is the diffusion coefficient of electrons in the film; $D_{S}$ is diffusion coefficient of substrate $\left(\mathrm{H}^{+}\right.$ions in this case) within the film; $k$ is the rate constant for the catalytic reaction; $\kappa_{A}$ is the partition coefficient of $\mathrm{H}^{+}$ions between solution and film (unitless); $C_{A}^{0}$ is the initial concentration of substrate in the bulk solution. Equation 4.1 also applies when the solvent is the substrate, i.e. where substrate diffusion within the film is not limiting. ${ }^{222}$

$$
\begin{aligned}
& d_{f}^{o p t}=\sqrt{\frac{D_{e}}{k \kappa_{A} C_{A}^{0}}} \\
& d_{f}^{o p t}=\sqrt{\frac{D_{S}}{k C_{A}^{0}}}
\end{aligned}
$$

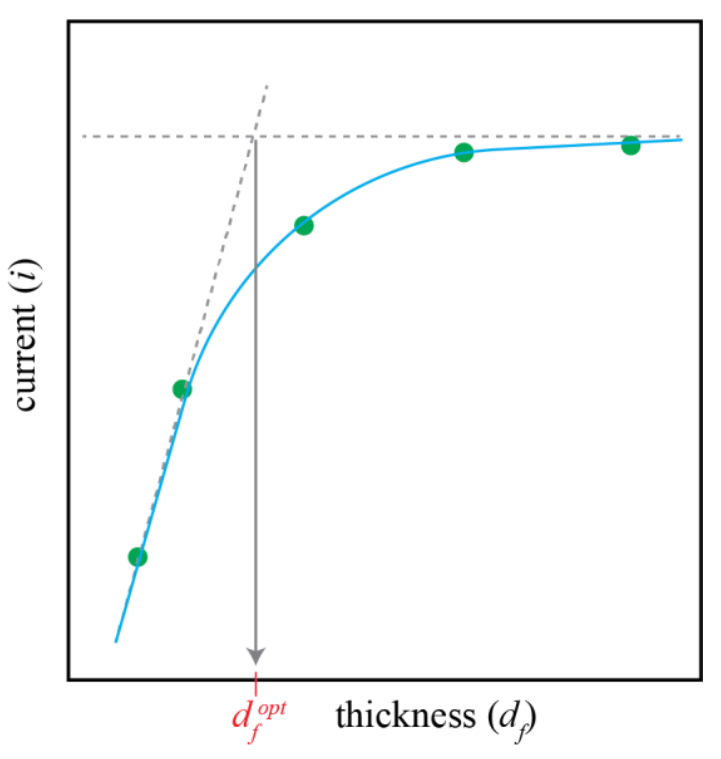

Figure 4.8 Illustration of a current versus thickness trend for an electrocatalytic film that follows the assumptions in the reports by Savéant and Costentin. ${ }^{222-224}$ The green circles are the current at a defined potential from cyclic voltammetry measurements. The dashed lines show the intersection between the linear portion at the beginning of the curve and the plateau region. The thickness at this intersection is the optimum film thickness. 


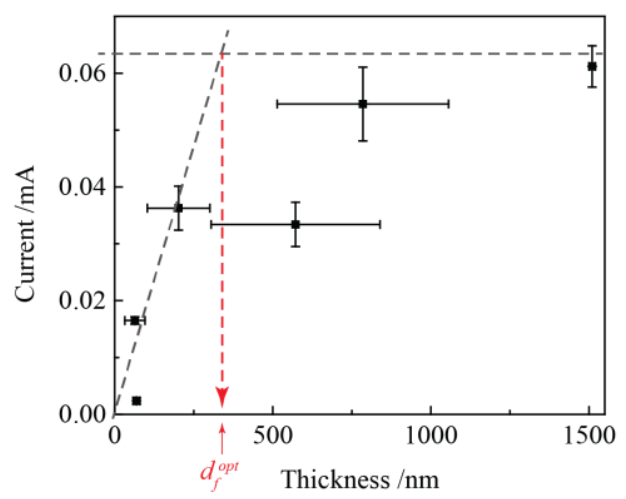

Figure 4.9 Replotted data from Figure 4.4c with gray dashed lines corresponding to the linear portion at the lower end of the curve and the plateau current. The intersection of these dashed lines is at the optimum thickness $\boldsymbol{d}_{\boldsymbol{f}}^{\text {opt }}$.

To determine whether the RGO/1 system obeys this model, the value for $d_{f}^{\text {opt }}$ was first determined from the data in Figure 4.4c. These data are replotted in Figure 4.9 with a linear fit for the data points for the thinnest films. From this figure, it is seen that $d_{f}^{\text {opt }}$ is approximately 300 $\mathrm{nm}$. Subsequently, $d_{f}^{\text {opt }}$ was calculated using Equations 4.1 and 4.2, with the parameters estimated in the following manner. $D_{S}$ was approximated based on literature values. The upper limit was set at $10^{-6} \mathrm{~cm}^{2} / \mathrm{s}$ based on a published diffusion coefficient of protons along the surface of a graphene sheet ${ }^{249}$ and the lower limit was set at $10^{-12} \mathrm{~cm}^{2} / \mathrm{s}$ based on published diffusion coefficients of $\mathrm{Li}^{+}$ ions in graphite. ${ }^{250} \kappa_{A}$ was estimated by using the reported value of 0.93 for a GO film. ${ }^{251}$ Assuming the value of $\kappa_{A}$ for RGO does not differ by more than an order of magnitude, this value is a good approximation. The value of $k C_{A}^{0}$ was calculated using the foot-of-the-wave (FOWA) method from a cyclic voltammogram of surface-adsorbed 1 on RGO in $2 \mathrm{mM}$ trifluoroacetic acid solution (Figure 4.10). The value obtained was $1.5 \times 10^{-5} \mathrm{~cm} / \mathrm{s}$. Lastly, the value of $D_{e}$ was calculated from the conductivity of RGO (reported in Chapter 2 of this thesis as $10^{4} \mathrm{~S} / \mathrm{m}$ ) as follows. Conductivity is related to mobility of electrons:

$$
\sigma=e n \mu_{e}
$$

where $\sigma$ is conductivity; $e$ is the charge of an electron $\left(1.602 \times 10^{-19} \mathrm{C}\right) ; n$ is the density of mobile electrons, estimated to be $10^{20} \mathrm{~cm}^{-3}$ based on literature ${ }^{252}$ and $\mu_{e}$ is the mobility of electrons. Using Equation 4.3 and the values listed for each parameter, the mobility of electrons in these RGO films is about $6.2 \mathrm{~cm}^{2} \mathrm{~V}^{-1} \mathrm{~s}^{-1}$. Following this, the diffusion coefficient of electrons can be calculated using the Einstein Relation: 


$$
D_{e}=\frac{\mu_{e} k_{B} T}{e}
$$

where $k_{B}$ is the Boltzmann constant; $T$ is temperature; and $e$ and $\mu_{e}$ are the same as listed as above. Using Equation 4.4, $D_{e}$ is calculated to be $0.16 \mathrm{~cm}^{2} / \mathrm{s}$.

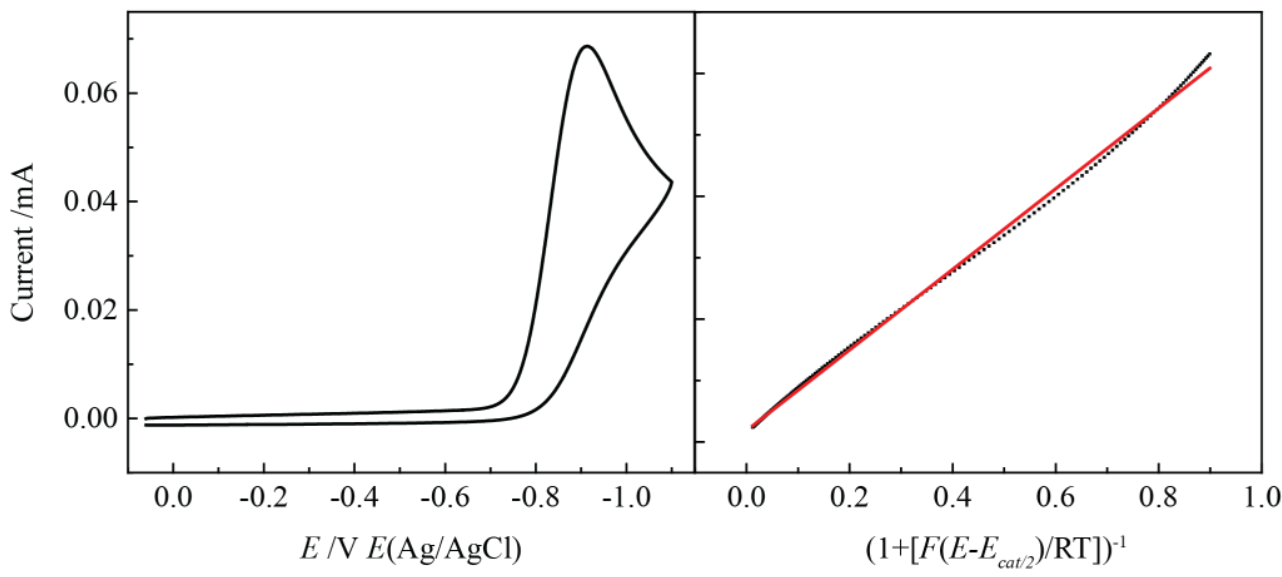

Figure 4.10 Left, cyclic voltammogram of surface adsorbed 1 on an RGO thin film on a glassy carbon electrode in deaerated $0.1 \mathrm{M} \mathrm{KPF}_{6}$ and $2 \mathrm{mM}$ trifluoroacetic acid at a scan rate of $50 \mathrm{mV}$ $\mathrm{s}^{-1}$. Right, FOWA fitting using the data from the voltammogram on the left. The black dotted line is the data and the red line is the linear fit. The value for $E_{\text {cat } / 2}$ was taken as $-0.82 \mathrm{~V}$ (the potential at half the current of the peak current). The slope of the line is $6.6 \times 10^{-5} \mathrm{~A}$, which is equal to $2.24\left(\frac{R T}{F v}\right)^{1 / 2}\left(2 k C_{A}^{0}\right)^{1 / 2}$.

Finally, $d_{f}^{o p t}$ can be calculated using the relationships in Equations 4.1 and 4.2. Using the former, $d_{f}^{o p t}$ is $108 \mathrm{~cm}$, and using the latter, $d_{f}^{o p t}$ is between $2.5 \mu \mathrm{m}$ and $2.5 \mathrm{~mm}$ (depending on the value used for $D_{S}$ ). The values for $d_{f}^{o p t}$ calculated in both cases are orders of magnitude different from the real value of $300 \mathrm{~nm}$, demonstrating that these equations are not good models for the system described here. Most likely, product inhibition is not accounted for in the Costentin \& Savéant model.

In the case where the film conductivity is assumed to be fast, instead of using $d_{f}^{o p t}$, the peak current, $I_{p}$, can be determined from film thickness (within the linear region of the current vs film thickness plot) based on the parameters described above. ${ }^{223}$ The relationship is shown in Equation 4.5.

$$
I_{p}=F k C_{p}^{0} \kappa_{A} C_{A}^{0} d_{f}
$$


The parameter $C_{p}^{0}$ is the concentration of catalyst sites within the film. For films used in Figure 4.4, that value is $2 \times 10^{-4} \mathrm{~mol} / \mathrm{cm}^{3}$. Estimated values for the other parameters are listed above in the description for use of Equations 4.1 and 4.2. Using $d_{f}=200 \mathrm{~nm}$ as an example, the value of $I_{p}$ calculated using Equation 4.5 is $5.3 \times 10^{-9} \mathrm{~A}$, far smaller than the approximately $3.8 \times 10^{-5} \mathrm{~A}$ in Figure 4.4.

In summary, assuming the values used for $D_{S}, D_{e}$, and $\kappa$ are good estimates, the data in Figure 4.4 do not agree with the models in any of these three reports. Therefore, it can be concluded that the electrocatalytic current in the system described here is not limited purely by substrate diffusion, catalytic rate, or electron diffusion. One possibility is that a combination of these factors limits the current. Another possibility is that there is a fourth factor that contributes to limiting current. This may be by product inhibition because of slow product $\left(\mathrm{H}_{2}\right)$ diffusion out of the film, bubble formation, or poisoning of the catalyst with $\mathrm{H}_{2}$ molecules. It may be possible to model product inhibition by addition of an expression or term in Equations 4.1, 4.2, and 4.5. Further work on this front should be pursued.

The data shown in Figure 4.6 help to further understand this system. These data were taken without varying the total amount of catalyst in the films. Because of the initial increase in TOF with film thickness in Figure 4.6, it is apparent that at least for thinner films, conductivity is not a limiting factor. Instead, we hypothesize that the increase in current and TOF (with no increase in the total amount of catalyst present) is due to a decrease in the concentration of catalyst within the film, which limits overlap of the diffusion radii of the catalyst sites.

For films thicker than about $500 \mathrm{~nm}$, the current and TOF start to decrease with increasing film thickness. Currently, there is more than one possible explanation for this phenomenon. First, conductivity of the films may start to play a role in limiting current in films thicker than about 500 $\mathrm{nm}$. Second, the structure or morphology of the film may be different with thicker dropcasted films compared to thinner ones. For example, thinner films may have a higher relative roughness, allow for easier penetration of substrate or escape of product. More characterization is necessary to determine the reason for this decrease in TOF with thicker films, but the data in Figure 4.7 helps to speak to this point.

In Figure 4.7, each of the three voltammograms are from films of equivalent thicknesses $(600 \pm 300 \mathrm{~nm})$ containing equivalent amounts of catalyst $\left(1.7 \pm 0.2 \times 10^{-10}\right.$ moles $)$. The black and red voltammograms are from films that also contain a $600 \pm 300 \mathrm{~nm}$ RGO film on top of and below 
the catalyst-containing film, respectively. Because the current in each of these voltammograms is equivalent and much lower than the blue curve, it suggests that at this film thickness, both substrate/product diffusion in the film and electron conduction within the film play essentially equivalent roles in limiting the total current. In other words, with an RGO film on top of the catalyst-embedded film, diffusion of species to and from the bulk solution is hindered; and with an RGO film below the catalyst-embedded film, conduction of electrons to catalyst sites within the film is hindered to an equal extent. Thereby the current from both electrodes in Figure 4.7 is similarly inhibited.

Future work. First, to definitively confirm that the data presented here do not follow the models in the reports by Savéant and Costentin, the estimated parameters should be measured. The value of $D_{e}$ for these RGO films can be determined by measuring the mobility with Hall or fieldeffect measurements on RGO thin films. Then the real value of $D_{S}$ can be measured by a variety of methods including electrochemical impedance spectroscopy, ${ }^{250}$ potential step chronoamperometry, ${ }^{250}$ or water permeability measurements. ${ }^{251} D_{e}$ and $D_{S}$ can then be compared directly to each other. The partition coefficient $\kappa$ can be measured by determining water uptake in an RGO film (by mass change). These three parameters should then be used to calculate the value of $d_{f}^{o p t}$ using Equations 4.1 and 4.2 and the value of $I_{p}$ using Equation 4.5.

Additionally, it should be determined whether morphological changes of the film may cause variations in limiting current for different film thicknesses. This can be analyzed by using statistical AFM imaging (i.e. many images over a large area of each film) or with mapping Raman spectroscopy. The distribution of catalyst within the film can be identified using mapping XPS with sputtering.

Since the data consistently suggests that a diffusional process controls the current in thinner films, experiments should be performed to determine whether $\mathrm{H}^{+}$diffusion or $\mathrm{H}_{2}$ diffusion is the limiting factor. This can be done in several ways. First, the ability of $\mathrm{H}_{2}$ to slow down the catalytic reaction should be determined. This can be done by performing electrocatalysis using 1 under an atmosphere of $\mathrm{H}_{2}$. The obtained currents can be compared to currents under an atmosphere of $\mathrm{N}_{2}$. If currents under $\mathrm{H}_{2}$ are lower than currents under $\mathrm{N}_{2}$, this is good evidence that buildup of product within the films can limit the electrocatalytic current. If the reaction is reversible under an atmosphere of $\mathrm{H}_{2}$, then this is evidence that product buildup specifically limits current by Le Chatelier's Principle. Second, $\mathrm{H}^{+}$diffusion may be limited by counterion (phosphate) diffusion 
within the RGO films. This can be evaluated by using different buffers with different sized anions. If buffers with smaller anions produce larger electrocatalytic currents, then this is evidence that $\mathrm{H}^{+}$ diffusion within the film primarily limits the current.

The data in Figures 4.4 and 4.6 suggest that the optimal film thickness is between 200 and $500 \mathrm{~nm}$ thick (the thickest film before TOF starts to decrease). Further experiments should keep film thickness constant within this range and vary the amount of catalyst within the film to find the optimum catalyst density. Factors affecting current may change depending on catalyst density; for example, in films with a high catalyst density, self-exchange of protons or electrons may occur, which may impact catalytic rate or transfer of electrons through the film.

Lastly, to be sure that catalytic rate is a not a limiting factor, kinetic isotope effect (KIE) experiments can be carried out on surface-adsorbed $\mathbf{1}$ and embedded $\mathbf{1}$ to determine whether the rate limiting step changes when $\mathbf{1}$ is embedded.

\subsection{Conclusions}

This chapter describes the kinetic factors that limit catalytic current in RGO thin films embedded with a molecular proton reduction electrocatalyst. Based on linear sweep voltammetry data, it was determined that film thickness plays a role in limiting catalytic current. Comparison of the data obtained here with previously published simulated voltammetry demonstrates that the electrocatalytic current in this system is not limited by a single process. Instead, multiple processes can affect the current simultaneously. Specifically, for films thinner than between 200 and 500 $\mathrm{nm}$, current is limited by diffusion of substrate or product into or out of the film, whereas for thicker films, RGO conductivity also plays a role in limiting catalytic current. For films that are $600 \pm 300 \mathrm{~nm}$ thick, electrical conductivity of RGO and diffusion of species within the film play essentially equal roles in limiting the catalytic current. The conclusions drawn from the data in this chapter apply to the conditions described here, i.e. in deaerated $0.2 \mathrm{M}$ phosphate buffer at $\mathrm{pH} 2$ and at $-0.67 \mathrm{~V}$ and $-0.95 \mathrm{~V}$ versus $\mathrm{Ag} \mid \mathrm{AgCl}$. Varying $\mathrm{pH}$, electrolyte identity and concentration, and applied potential may change the behavior of the electrocatalytic films. This is the first study on fundamental kinetics of proton reduction electrocatalysis in RGO thin films, and the results obtained here highlight several further directions that should be pursued. For example, increasing the porosity of the films to allow faster $\mathrm{H}^{+}$and $\mathrm{H}_{2}$ diffusion may allow for larger catalytic currents. Also, the counterion dependence of the current should be investigated, as this can be a critical factor. 


\section{Chapter 5. Discovery of an Unusually Stable Reduced Viologen via Synergistic Folding and Encapsulation}

Adapted from Journal of Electrochemical Society, Vol. 166, "Discovery of Unusually Stable Reduced Viologen via Synergistic Folding and Encapsulation," MacInnes, M. M.; Cousineau, B. R.; Youngs, S. M.; Sinniah, K.; Reczek, J. J.; and Maldonado, S. H825-H834, Copyright 2019.

\subsection{Introduction}

Viologens (1,1'-disubstituted-4,4'-bipyridinium ions) are an important class of redox species. They have received attention for use as electron acceptors in fundamental studies of chemical and electrochemical charge transfer, ${ }^{253-254}$ as the charge storage medium in redox flow batteries, ${ }^{255-260}$ as the active material in electrochromic devices, ${ }^{261-262}$ and as the redox-active component in molecular machines. ${ }^{263-266}$ Their appeal in these applications derives from their stability, highly reversible redox character, and relative ease of sythesis. ${ }^{255-262}$ Viologen dications reversibly undergo one-electron reductions to form highly stable radical cations. ${ }^{253-254}$ This transition is fast because it involves minimal bond length and angle change (i.e. the reorganization energy is low). ${ }^{254} \mathrm{~A}$ second one-electron reduction forms the neutral species, but this transition is not considered fully reversible in polar and protic solvents because of its low solubility in these solvents. ${ }^{254}$

A disadvantage of viologens in energy and electronic applications is the reactivity of the reduced viologen radicals with $\mathrm{O}_{2} \cdot{ }^{253,267-268}$ For species like methyl viologen radicals $\left(\mathbf{M V}^{\bullet+}\right)$, the rate constant for oxidation by $\mathrm{O}_{2}$ is large $\left(8 \times 10^{8} \mathrm{M}^{-1} \mathrm{~s}^{-1}\right),{ }^{269}$ effectively at the diffusion limit. Strategies to inhibit parasitic oxidation of reduced viologens by $\mathrm{O}_{2}$ are therefore of significant importance.

One potential approach to stabilize reduced viologens is by embedding them inside protective structures. ${ }^{137,}$ 143, 264, 270-277 In this vein, viologens have been inserted in polymers, ${ }^{138,278-}$ 288 micelles, ${ }^{270-271,273-275}$ and macromolecules. ${ }^{137,}$ 140, 289-290 In particular, inclusion complexes between viologens and cucurbit[n]urils $(\mathrm{CB}[\mathrm{n}])$, a class of rigid and water-soluble macrocyclic 
host molecules, have been shown to tune the redox potentials of viologens. ${ }^{143,264,272,276,291-293}$ However, enhanced stability of the reduced forms in the presence of $\mathrm{O}_{2}$ has yet to be shown.

In this work, we explore the concept that a hierarchical supramolecular assembly can form structures that inhibit parasitic reaction with $\mathrm{O}_{2}$. Herein, we report the design, assembly, and electrochemical behaviors of covalently-linked viologen dimers complexed with cucurbit[7]uril $(\mathrm{CB}[7])$ and cucurbit[8] uril $(\mathrm{CB}[8])$ in aqueous solution. The internal diameters of $\mathrm{CB}[7]$ and $\mathrm{CB}[8]$ are $11.33 \AA$ and $12.95 \AA$, respectively. ${ }^{294}$ To avoid confusion, in this report the term "dimer" refers strictly to two viologens covalently bound through a defined linker. Two specific linkers are explored: an ortho-benzyl group (2) and a propyl group (3) (Figure 5.1). These linkers space two methyl viologen units by the similar short $N-N$ ' distance of approximately $5 \AA$ but differ in equilibrium geometry and linker rigidity. The electrochemical and spectroscopic data presented here indicate that when these viologen dimers are reduced in the presence of cucurbit[8]uril $(\mathrm{CB}[8])$, a unique and unusually stable monoradical species is formed. We show that specifically the combination of the structure of the viologen dimer with subsequent encapsulation by $\mathrm{CB}[8]$ is required to form a stabilized, reduced radical. Further, data are presented that distinguish these stabilized species from viologen "pimers" which have been extensively studied previously. 254,295 299 This is the first time that a monoradical viologen dimer has been isolated through use of supramolecular assemblies, and it is the first report of a solubilized viologen radical species that is significantly stabilized in the presence of oxygen.
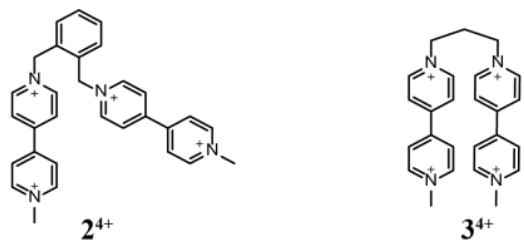

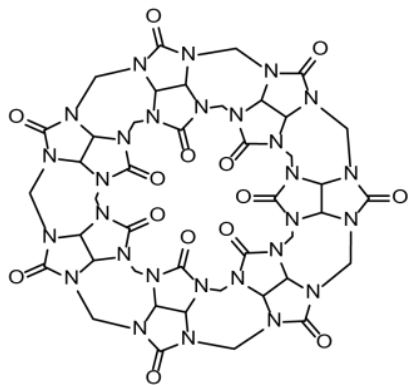

$\mathrm{CB}[7]$

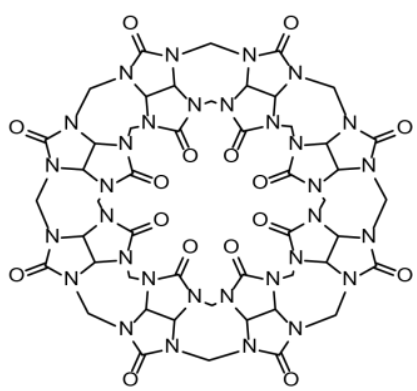

$\mathrm{CB}[8]$

Figure 5.1 Depictions of $\mathbf{2}^{4+}, \mathbf{3}^{4+}, \mathrm{CB}[8]$, and $\mathrm{CB}[7]$. 


\subsection{Experimental.}

Chemicals and materials. Anhydrous dibasic sodium phosphate (Sigma Chemical Company), monobasic potassium phosphate (99.6\%, J.T. Baker, Inc.), sodium dithionite ( $\geq 82 \%$, Sigma Aldrich), cucurbit[7]uril hydrate (CB[7], Sigma Aldrich), cucurbit[8] uril hydrate (Sigma Aldrich), iodomethane (99\%, Sigma Aldrich), 4,4'-bipyridine (98\%, Sigma Aldrich), DMF ( $\geq 99.8 \%$, Sigma Aldrich), $\alpha, \alpha$ '-dibromo-o-xylene (97\%, Sigma Aldrich), and deuterated water (99.8 atom \%, Acros Organics) were used as received. Water with a resistivity $>18.2 \mathrm{M} \Omega$ (Nanopure Barnstead Water Purification) was used throughout. Glassy carbon electrodes were prepared by first polishing with alumina $(1 \mu \mathrm{m}, 0.3 \mu \mathrm{m}$, and $0.05 \mu \mathrm{m}$ alumina suspensions $)$ on felt and then sonicating in methanol for 30 minutes. A carbon cloth electrode was used for bulk electrolysis and was prepared by thoroughly rinsing with clean water. Platinum mesh was used as a counter electrode. For bulk electrolysis experiments, the platinum mesh was isolated from the working electrode by a frit.

Synthesis of N-Methyl-4,4'-bipyridinium iodide. Iodomethane (1.20 mL, $19.2 \mathrm{mmol}, 1.0$ eq.) was added to 4,4'-bipyridine (2.9994 g, $19.2 \mathrm{mmol}, 1.0$ eq.) in $\mathrm{CH}_{2} \mathrm{Cl}_{2}$ (50 mL). The solution was stirred at room temperature for 24 hours. A bright yellow solid precipitated out. The solid was filtered and any residual 4,4'-bipyridine washed away with diethyl ether ( 3 x $10 \mathrm{ml}$ ), yielding $N$ Methyl-4,4'-bipyridinium iodide (86.5 \%, $4.9497 \mathrm{~g}) .{ }^{1} \mathrm{H}$ NMR of the solid showed $>99 \%$ with no trace of starting material, and the collected solid was used directly in the synthesis of $\mathbf{2}$ and $\mathbf{3}$.

Synthesis of 2. N-Methyl-4,4'-bipyridinium iodide (0.6035 g, $2.0 \mathrm{mmol}, 2.2$ eq.) was reacted with $\alpha, \alpha^{\prime}$-dibromo-o-xylene (0.2432 g, $0.92 \mathrm{mmol}, 1.0$ eq.) in DMF (30 mL). The solution was stirred at $60^{\circ} \mathrm{C}$ for 3 days with a rubber septum and vent needle. An orange product precipitated out. The product was filtered and washed with DMF $(2 \times 10 \mathrm{~mL})$ to completely remove any residual $\mathrm{N}$-Methyl-4,4'-bipyridinium iodide, yielding 1 (87.0\%, $0.6893 \mathrm{~g}) .{ }^{1} \mathrm{H}$ NMR (400 $\left.\mathrm{MHz}, \mathrm{D}_{2} \mathrm{O}\right) \delta 9.194(\mathrm{~d}, J=6.8 \mathrm{~Hz}, 4 \mathrm{H}), 9.112(\mathrm{~d}, J=6.4 \mathrm{~Hz}, 4 \mathrm{H}), 8.669(\mathrm{~d}, J=6.8 \mathrm{~Hz}, 4 \mathrm{H}), 8.598(\mathrm{~d}$, $J=6.8 \mathrm{~Hz}, 4 \mathrm{H}), 7.695(\mathrm{~d}, J=9.2 \mathrm{~Hz}, 2 \mathrm{H}), 7.423(\mathrm{~d}, J=9.2 \mathrm{~Hz}, 2 \mathrm{H}), 6.229(\mathrm{~s}, 4 \mathrm{H}), 4.554(\mathrm{~s}, 6 \mathrm{H})$ ppm; ${ }^{13} \mathrm{C}$ NMR (400 MHz, D $\left.2 \mathrm{O}\right) \delta 151.066,149.571,146.438,145.901,131.451,130.981$, 130.837, 127.579, 126.908, 61.362, 48.531 ppm. Analysis by analytical HPLC showed > 99\% purity of $\mathbf{1}$ in the recovered solid. A schematic depiction of the synthetic protocol and the ${ }^{1} \mathrm{H} \mathrm{NMR}$, ${ }^{13} \mathrm{C}$ NMR spectra are shown in Figures 5.2-5.4 respectively. 
Synthesis of 3. $N$-Methyl-4,4'-bipyridinium iodide ( $0.6163 \mathrm{~g}, 2.1 \mathrm{mmol}, 2.2 \mathrm{eq}$.) was added to 1,3-dibromopropane ( $0.094 \mathrm{~mL}, 0.93 \mathrm{~mol}, 1.0$ eq.) in $\mathrm{DMF}(30 \mathrm{~mL})$. The solution was stirred at $60^{\circ} \mathrm{C}$ for 3 days with a rubber septum and vent needle. A yellow product precipitated out. The product was filtered and washed with DMF $(3 \times 10 \mathrm{~mL})$ to completely remove any residual $\mathrm{N}$ Methyl-4,4'-bipyridinium iodide, yielding 3 (35\%, $0.258 \mathrm{~g}$ ). ${ }^{1} \mathrm{H}$ NMR (400 MHz, $\left.\mathrm{D}_{2} \mathrm{O}\right) \delta 9.098(\mathrm{~d}$, $J=6.8 \mathrm{~Hz}, 4 \mathrm{H}), 8.936(\mathrm{~d}, J=6.8 \mathrm{~Hz}, 4 \mathrm{H}), 8.484(\mathrm{~d}, J=6.8 \mathrm{~Hz}, 4 \mathrm{H}), 8.408(\mathrm{~d}, J=6.8 \mathrm{~Hz}, 4 \mathrm{H})$, $4.873(\mathrm{t}, J=8.0 \mathrm{~Hz}, 4 \mathrm{H}), 4.368(\mathrm{~s}, 6 \mathrm{H}), 2.859(\mathrm{~m}, J=7.9 \mathrm{~Hz}, 2 \mathrm{H}) \mathrm{ppm} ;{ }^{13} \mathrm{C} \mathrm{NMR}\left(400 \mathrm{MHz}, \mathrm{D}_{2} \mathrm{O}\right)$ $\delta 150.817,149.677,146.409,145.805,127.522,126.841,58.306,48.483,31.905,30.324$ ppm. Analysis by analytical HPLC showed $>99 \%$ purity of $\mathbf{1}$ in the recovered solid. A schematic depiction of the synthetic protocol and the ${ }^{1} \mathrm{H}$ NMR, ${ }^{13} \mathrm{C}$ NMR spectra are shown in Figures 5.25.4 respectively.

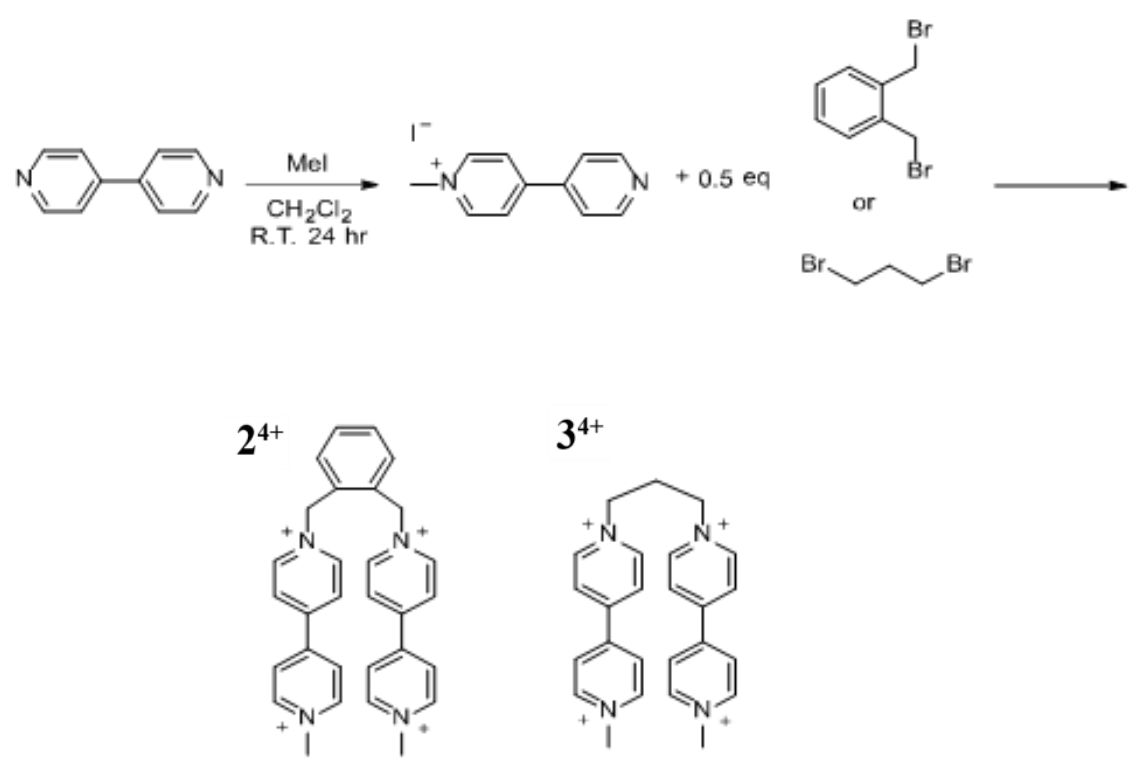

Figure 5.2 Syntheses of $\mathbf{2}^{4+}$ and $\mathbf{3}^{4+}$. Details for these syntheses are described below. 

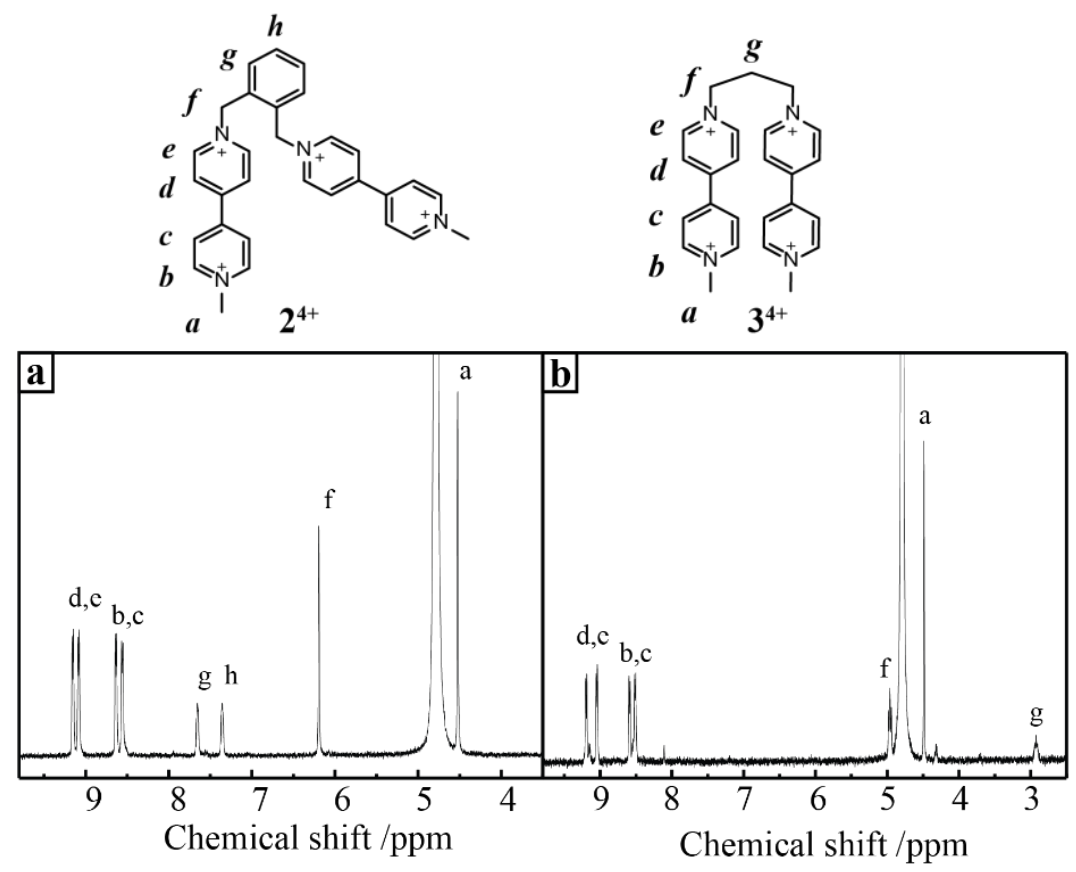

Figure 5.3 ${ }^{1} \mathrm{H}$ NMR spectra of a) an aqueous $\mathrm{D}_{2} \mathrm{O}$ solution of $\mathbf{2}^{4+}$ and b) an aqueous $\mathrm{D}_{2} \mathrm{O}$ solution of $\mathbf{3}^{4+}$. The peaks are labeled corresponding to the protons in the structures above.

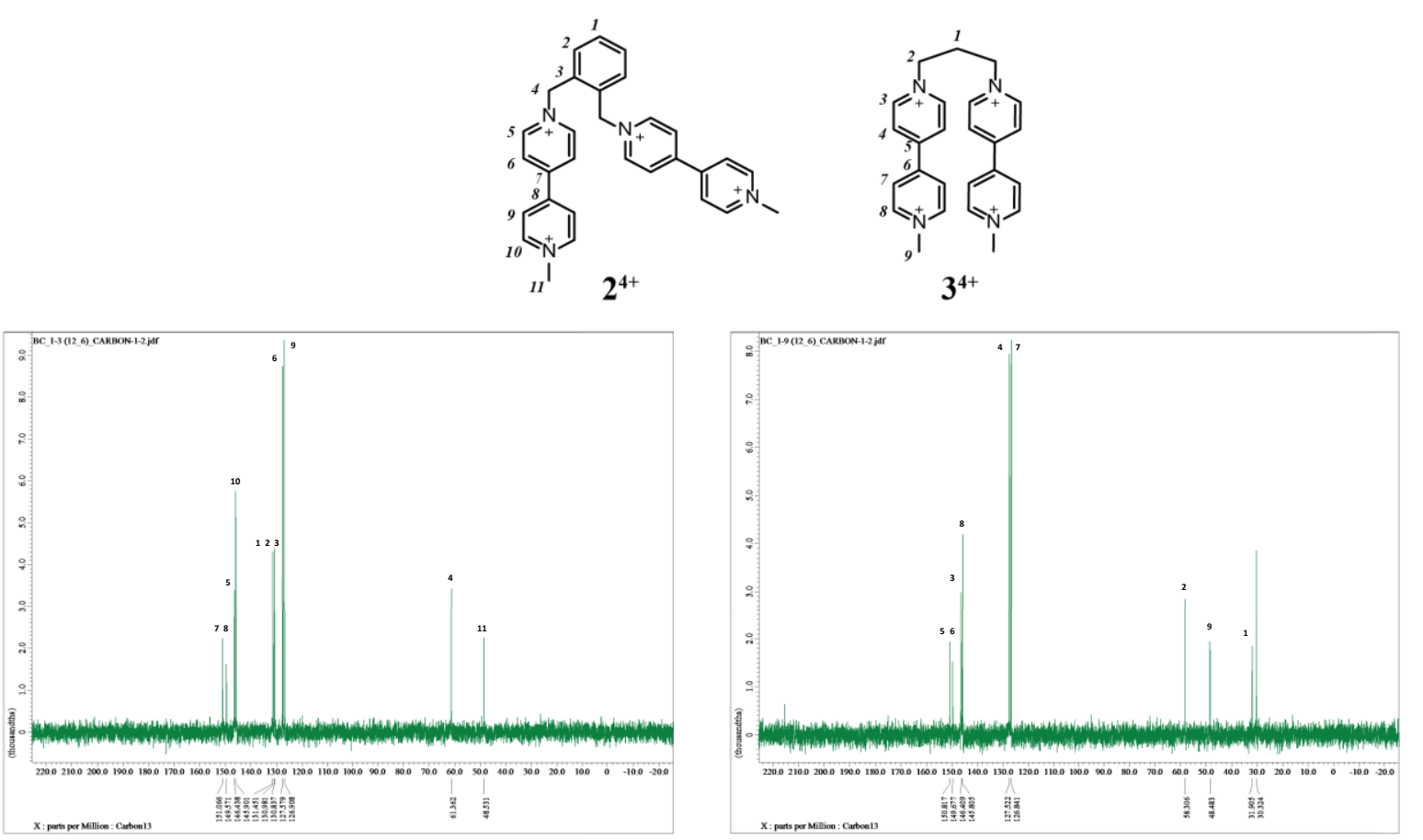

Figure 5.4 ${ }^{13} \mathrm{CNMR}$ spectra of $\mathbf{2}^{4+}$ (left) and $\mathbf{3}^{4+}$ (right). The peaks are labeled corresponding to the carbon atoms in the structures above. 
Preparation of viologen-cucurbituril mixtures. All viologen-cucurbituril solutions were prepared by first dissolving the appropriate amount of viologen in water $\left(\right.$ or $\left.\mathrm{D}_{2} \mathrm{O}\right)$ or buffer solution. Cucurbituril was then added in excess and the solution was stirred for at least 2 hours. The undissolved cucurbituril was filtered out using a $0.2 \mu$ m nylon syringe filter. Based on NMR integrations, this resulted in a $1: 1$ ratio of $\mathbf{2}^{4+}: \mathrm{CB}[8]$; a $1: 1$ ratio of $\mathbf{3}^{4+}: \mathrm{CB}[8]$; a $1: 3$ ratio of $\mathbf{2}^{4+}: \mathrm{CB}[7]$; and a $1: 3$ ratio of $\mathbf{3}^{4+}: \mathrm{CB}[7]$.

Isothermal titration calorimetry (ITC). ITC data was obtained on a Nano ITC Standard Volume instrument from TA Instruments. Experiments were performed in 10 mmol sodium phosphate buffer and all solutions were degassed for $25 \mathrm{~min}$ at room temperature prior to running ITC experiments. The reference cell in the ITC was filled with degassed ultrapure water (18.2 $\mathrm{M} \Omega$ $\mathrm{cm}^{-1}$ resistivity). Experiments were run in multiple-injection mode with 20 injections ( $12 \mu \mathrm{L}$ each) with $1000 \mathrm{~s}$ injection intervals. The stir rate was $250 \mathrm{rpm}$. Once the cell had been filled and the syringe loaded, stirring was turned on and the apparatus was allowed to autoequilibrate. All data were recorded with the TA Instruments software provided, ITCRun version 2.2.3. The area under each peak was integrated, and the resulting data were further analyzed using TA NanoAnalyze version 2.4.1, fitting to a 1:1 binding model of dimer to cucurbituril.

Absorbance Measurements. UV-visible-NIR absorbance spectra were obtained using a Varian Carey 5000 UV-vis-NIR spectrophotometer. Solutions of $0.25 \mathrm{mM}$ viologen with and without approximately 1 equivalent of cucurbituril were electrolyzed and immediately syringed into a $1-\mathrm{cm}$ quartz cuvette sealed with a septum that had been flushed with $\mathrm{N}_{2}$. The supporting electrolyte was deaerated $0.075 \mathrm{M}$ phosphate buffer ( $\mathrm{pH} \sim 7$ ) using sodium monohydrogen phosphate and potassium dihydrogen phosphate. Backgrounds were taken using plain electrolyte solutions. Absorbance changes upon exposure to $\mathrm{O}_{2}$ was monitored using an Analytik Jena SPECORD S600 spectrophotometer. Solutions used for these experiments were $30 \mu \mathrm{M}$ viologen in $0.075 \mathrm{M}$ phosphate buffer ( $\mathrm{pH}$ 7). Solutions containing cucurbituril were prepared by dissolving excess cucurbituril in the buffered viologen solution, stirring for at least two hours and then filtering through a $0.2 \mu \mathrm{m}$ nylon syringe filter to remove undissolved cucurbituril. These solutions were electrolyzed to $100 \%$ under a stream of nitrogen and then the electrodes and nitrogen stream were removed and an immersion probe (Hellma Analytics) was quickly inserted. Spectra were recorded using the immersion probe while stirring the solution at a constant speed. 
Electrochemical measurements. Electrochemical measurements were taken using a $\mathrm{CH}$ Instruments $760 \mathrm{C}$ potentiostat. A three-electrode cell was used with a supporting electrolyte of deaerated 0.075 $\mathrm{M}$ phosphate buffer $(\mathrm{pH} \sim 7)$ as described above. A platinum mesh was used as the counter electrode and $\mathrm{Ag} \mid \mathrm{AgCl}$ was used as the reference electrode. The working electrode for cyclic voltammetry experiments was glassy carbon (geometric surface area $0.071 \mathrm{~cm}^{2}$ ) and for electrolysis of bulk solutions was a carbon cloth electrode.

${ }^{1}$ H NMR spectroscopy. Measurements were conducted on a Varian MR 400 instrument at room temperature and were referenced against the solvent peak. Measurements were performed on $1 \mathrm{mM}$ viologen solutions in $\mathrm{D}_{2} \mathrm{O}$ with and without approximately 1 equivalent of cucurbituril. Sodium dithionite was used to reduce the solutions.

Molecular Orbital Calculations Molecular modeling was carried out using Spartan '16 for Macintosh. All structures were constructed in Spartan and equilibrium geometries were calculated for each redox state independently (4+, no unpaired elections; $3+$, one unpaired electron; $2+$, two unpaired electrons) for dimers $\mathbf{2}$ and $\mathbf{3}$ and the corresponding complexes of the dimers and CB[8]. All geometry calculations were initiated from a randomized starting point and carried out using density functional theory in water solvent with a B3LYP functional at the 6-31G* level. The calculated equilibrium structures were then used in energy calculations to obtain orbital and spinstate information using density functional theory in water solvent with a B97D functional at the 6$311+\mathrm{G}^{* *}$ level. The B97D functional was used to achieve energy calculations that more adequately include dispersion stabilization of the delocalized radicals. ${ }^{55}$

\subsection{Results.}

Dimer Conformers. Molecular modeling was performed to determine the relevant conformers of $\mathbf{2}$ and 3 . The results indicated that dimers $\mathbf{2}^{\mathbf{4 +}}$ and $\mathbf{3}^{\mathbf{4 +}}$ prefer to adopt extended conformations in water due to charge-repulsion (Figure 5.5). In contrast, the reduced dimers $\mathbf{2}^{\mathbf{2 +}}$ and $3^{2+}$ assemble into folded structures that balance charge-repulsion with $\pi$-stacked stabilization of the radical species (so-called pimers). While $\mathbf{2}^{\mathbf{2 +}}$ and $\mathbf{3}^{\mathbf{2 +}}$ adopt conformations with similar distances between the viologen groups, the equilibrium geometry structure calculated for the rigid $o$-xylene-linked $\mathbf{2}^{\mathbf{2}}$ had near-perfect alignment of overlapping viologen surfaces, while propyllinked $\mathbf{3}^{\mathbf{2}}$ displayed a significantly skewed relative orientation of the viologen groups (Figure 5.6). 


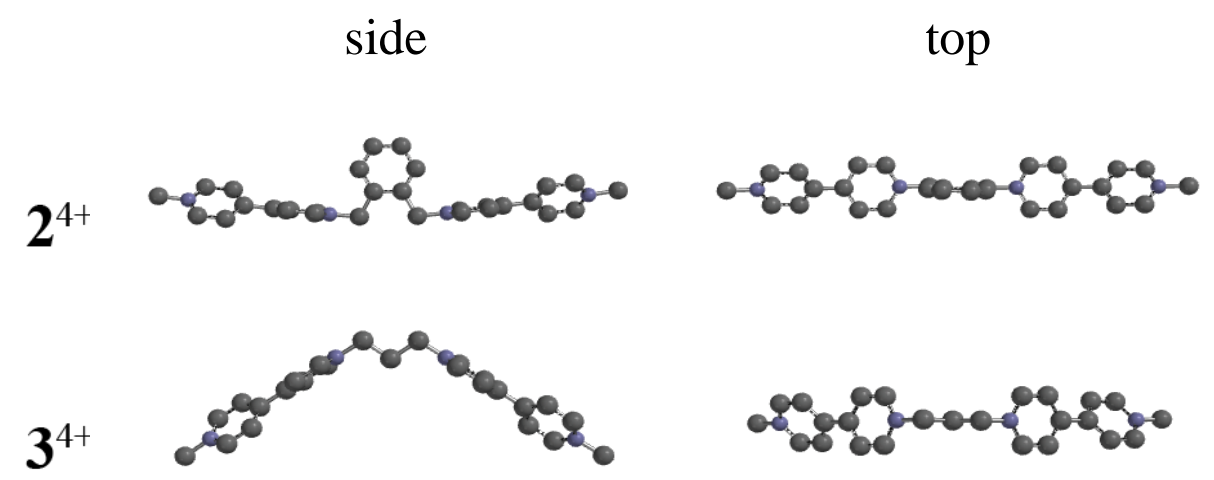

Figure 5.5 Equilibrium geometries modeled for free reduced dimers $\mathbf{2}^{4+}$ and $\mathbf{3}^{4+}$. Note the clear difference in the number of bond rotations needed to access the $\pi$-stacked geometries from these conformations: $\mathbf{2}^{4+}<\mathbf{3}^{4+}$

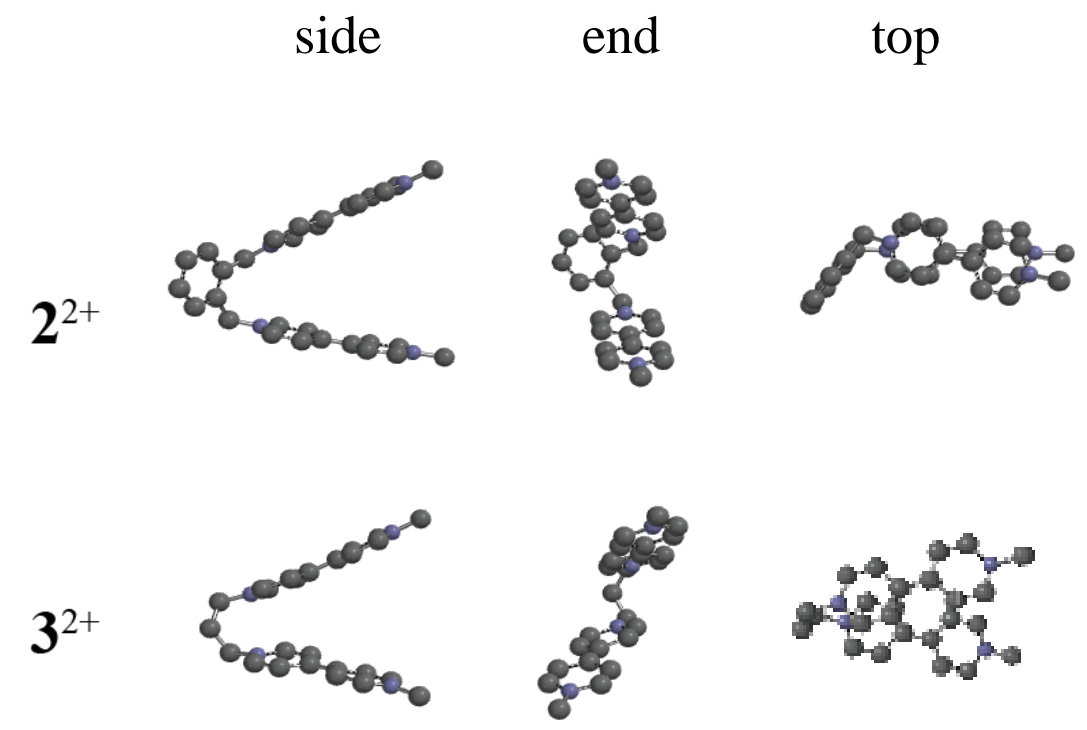

Figure 5.6 Equilibrium geometries modeled for free reduced dimers $\mathbf{2}^{2+}$ and $\mathbf{3}^{2+}$. Side view shows similar spacing of viologen groups, while end and top view illustrate the better $\pi-\pi$ stacking preorganization of $\mathbf{2}^{2+}$. 

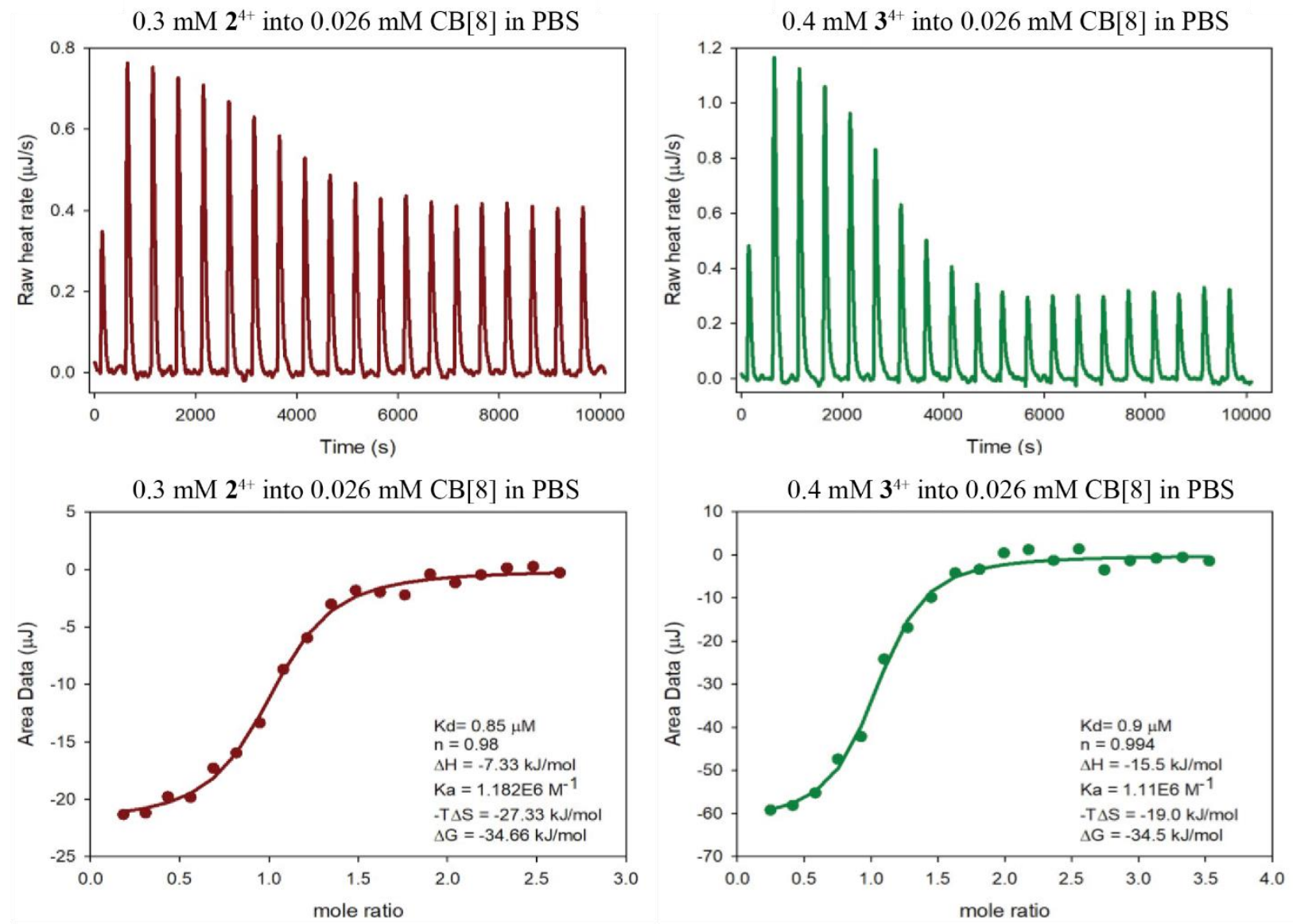

Figure 5.7 ITC determination of binding constant between $\mathbf{2}^{4+}$ and CB[8] (left) and $\mathbf{3}^{4+}$ (right). All solutions for titration were made in phosphate buffer $(10 \mathrm{mmol})$ and degassed in vacuum. Solutions of $2^{4+}(0.3 \mathrm{mM})$ or $3^{4+}(0.4 \mathrm{mM})$ were titrated into a solution of $\mathrm{CB}[8](0.026 \mathrm{mM})$, and the resulting exotherm curve was fit to obtain binding constants of $1.2 \times 10^{6} \mathrm{M}^{-1}$ for $2^{4+}$ with $\mathrm{CB}[8]$ and $1.1 \mathrm{x}$ $10^{6} \mathrm{M}^{-1}$ for $3^{4+}$ with $\mathrm{CB}[8]$, respectively.

Isothermal Calorimetry. ITC measurements (Figure 5.7) were performed on both viologen dimers in the 4+ oxidation state in the presence of $\mathrm{CB}[8]$ in a phosphate buffer. Binding constants between $2^{4+}$ and $\mathrm{CB}[8]$ and between $3^{4+}$ and $\mathrm{CB}[8]$ calculated from this data were $1.18 \times 10^{6} \mathrm{M}^{-1}$ and $1.11 \times 10^{6} \mathrm{M}^{-1}$, respectively, indicating a strong affinity of $\mathrm{CB}[8]$ for both viologens.

Cyclic Voltammetry. The cyclic voltammograms of $\mathbf{2}^{4+}, \mathbf{3}^{4+}$, and $\mathbf{M V}^{2+}$ in the presence and absence of $\mathrm{CB}[7]$ and $\mathrm{CB}[8]$ are shown in Figure 5.8. Scanning cathodically from open circuit potential, MV and compound 2 showed single reversible redox waves in the absence of cucurbituril. The voltammogram of compound $\mathbf{3}$ produced a second reversible wave at a lower current density slightly negative of the main redox wave. The $E_{1 / 2}$ values and peak potential separations $\left(\Delta E_{p}\right)$ of each of these redox waves are listed in Table 5.1. The wave for MV was consistent with the 1- 
electron $\mathbf{M V}^{2+/ 1+}$ transition. ${ }^{253} T$ The $E_{1 / 2}$ for the redox wave of $\mathbf{2}$ and the major wave of $\mathbf{3}$ were much more positive than MV with lower current densities and smaller peak separations. The lower current densities were attributed to smaller diffusion coefficients due to the bulkier nature of the molecules (5.6 × $10^{-6}$ and $8.6 \times 10^{-6} \mathrm{~cm}^{2} \mathrm{~V}^{-1} \mathrm{~s}^{-1}$, for $\mathbf{2}^{4+}$ and $\mathbf{M V}^{2+}$, respectively). The more positive reduction potentials are consistent with favored pimer formation due to the forced proximity of two viologen units on each molecule. ${ }^{270,300-302}$ Lastly, the small peak separations for compounds $\mathbf{2}$ and $\mathbf{3}$ are consistent with 2-electron redox processes, and therefore these waves were ascribed to the $\mathbf{2}^{4+/ 2+}$ and the $\mathbf{3}^{4+/ 2+}$ transitions. ${ }^{30,303}$ The second redox wave in the voltammogram of $\mathbf{3}$ at about $-0.61 \mathrm{~V}$ is likely due to effects from a higher degree of freedom for rotation around the propyl linker as compared to the benzyl linker in $\mathbf{2}$. In other words, a fraction of $\mathbf{3}^{4+}$ exists in a conformation that disfavors pimer formation, resulting in a more negative reduction potential.

Addition of $\mathrm{CB}$ [7] to 2, 3, and MV lowered the peak currents and shifted the $E_{1 / 2}$ values negative while significantly increasing the peak separations in $\mathbf{2}$ and $\mathbf{3}$. The lower peak currents were because of reduced diffusivity of the viologens when encapsulated in the large $\mathrm{CB}[7]$ scaffold. The negative shift in reduction potentials has previously been ascribed to the inability of the $\mathrm{CB}$ [7] cavity to accommodate two viologen units. ${ }^{272,276,291}$ Complexation with $\mathrm{CB}$ [7] therefore inhibits dimer formation. This is also the reason for the increased $\Delta E_{p}$ values. Inhibited pimer formation suggests that the second reductions of $\mathbf{2}$ and $\mathbf{3}$ are more difficult that the first reductions, i.e. $2^{4+/ 3+}$ occurs at a more positive potential than $2^{3+/ 2+}$. Because the redox wave still appeared as a single wave, the separation between $E_{1 / 2}$ for $2^{4+/ 3+}$ and $E_{1 / 2}$ for $2^{3+/ 2+}$ was small, less than about $120 \mathrm{mV}$. ${ }^{303}$ The same was true for $\mathbf{3}$ in $\mathrm{CB}[7]$. Furthermore, encapsulation in $\mathrm{CB}[7]$ is the reason for the absence of the secondary redox wave in the voltammogram of $3 / \mathrm{CB}$ [7]. The two redox waves in the voltammogram of $\mathbf{3}$ were due to the existence of two conformers in solution: one being the preferred orientation for pimer formation and the other being a non-preferred orientation for pimer formation. Since pimer formation is precluded in the presence of $\mathrm{CB}$ [7], these two conformations have the same reduction potential and only one redox wave was observed in the voltammogram of $\mathbf{3 / C B}[7]$.

Addition of $\mathrm{CB}$ [8] to each of the three compounds shifted the potentials of each redox wave positive. This positive shift can be ascribed to the facilitation of pimer formation within the $\mathrm{CB}[8]$ cavity. ${ }^{143,276}$ Interestingly, the voltammograms of $2 / \mathrm{CB}[8]$ and $3 / \mathrm{CB}[8]$ no longer showed single 


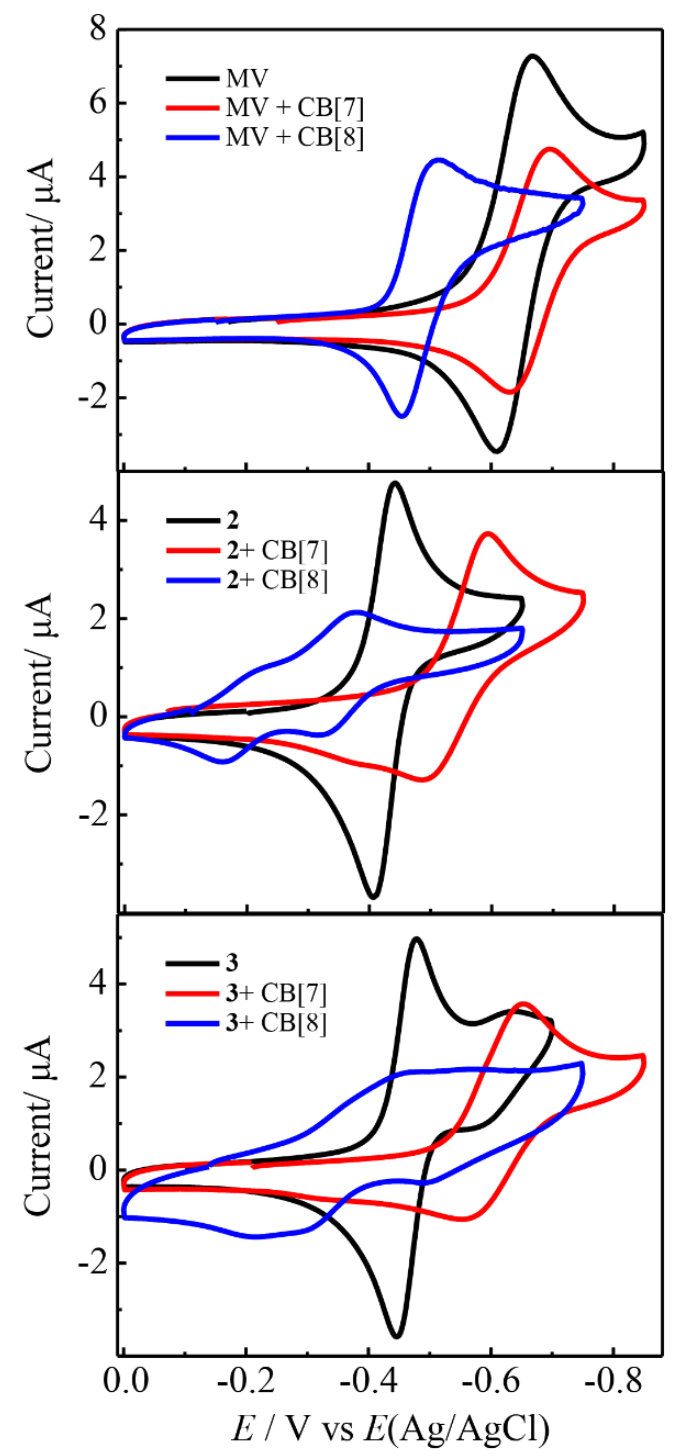

Figure 5.8 Cyclic voltammetry of viologen/cucurbituril pairs. From top to bottom: MV; 2 ; 3; MV $+\mathrm{CB}[7] ; \mathbf{2}+\mathrm{CB}[7] ; \mathbf{3}+\mathrm{CB}[7] ; \mathbf{M V}+\mathrm{CB}[8] ; \mathbf{2}+\mathrm{CB}[8] ; \mathbf{3}+\mathrm{CB}[8]$. Concentrations of MV, $\mathbf{2}$, and 3 , were $0.4,0.2$, and $0.2 \mathrm{mM}$, respectively. Concentrations of $\mathrm{CB}$ [7] and $\mathrm{CB}$ [8] were equivalent to the respective viologen in each panel. All voltammetry was performed in dearated $0.075 \mathrm{M}$ phosphate buffer at $\mathrm{pH}=7$ at a glassy carbon electrode and a scan rate of $0.05 \mathrm{~V} \mathrm{~s}^{-1}$.

reversible redox waves. The voltammogram of $2 / \mathrm{CB}[8]$ contained two apparently reversible redox waves with $\Delta E_{p}$ values larger than that of 2 but smaller than that of $2 / \mathrm{CB}[7]$. The current densities of these waves were much lower than in any of the previous voltammograms. Integration of these waves showed that the sum of the charge passed during both processes was approximately equivalent to the charge passed during the single redox process in either 2 or $2 / \mathrm{CB}$ [7]. These data 
then suggest that the two redox waves in $2 / \mathrm{CB}[8]$ correspond to the 1 -electron $2^{4+/ 3+}$ and $2^{3+/ 2+}$ transitions. The voltammogram of $\mathbf{3 / C B}[8]$ did not have two well-defined reversible waves like that of $2 / \mathrm{CB}[8]$; however it is apparent that more than one redox process occurred and that these processes were generally shifted positive of the redox process for 3 . Because the redox waves were not well-defined and reversible they are not listed in Table 5.1. The quasi-reversibility of the waves in $3 / \mathrm{CB}[8]$ can be attributed, as described above, to the higher degrees of rotational freedom afforded by the propyl linker.

\begin{tabular}{|c|c|c|c|c|}
\hline Compound & $E_{1 / 2}^{a, c} / \mathrm{V}$ & $\Delta E_{p}{ }^{a} / \mathrm{V}$ & $E_{1 / 2^{b, c} / \mathrm{V}}$ & $\Delta E_{p}^{b} / \mathrm{V}$ \\
\hline MV & $-0.638 \pm 0.002$ & $0.058 \pm 0.002$ & -- & -- \\
\hline 2 & $-0.424 \pm 0.002$ & $0.036 \pm .002$ & -- & -- \\
\hline 3 & $-0.462 \pm 0.002$ & $0.032 \pm 0.002$ & $-0.61 \pm 0.01$ & $0.050 \pm 0.010$ \\
\hline $\mathbf{M V}+\mathrm{CB}[7]$ & $-0.663 \pm 0.002$ & $0.065 \pm 0.003$ & -- & -- \\
\hline $\mathbf{2}+\mathrm{CB}[7]$ & $-0.540 \pm 0.002$ & $0.107 \pm 0.002$ & -- & -- \\
\hline $\mathbf{3}+\mathrm{CB}[7]$ & $-0.603 \pm 0.003$ & $0.100 \pm 0.003$ & -- & -- \\
\hline $\mathbf{M V}+\mathrm{CB}[8]$ & $-0.484 \pm 0.004$ & $0.061 \pm 0.004$ & -- & -- \\
\hline $\mathbf{2}+\mathrm{CB}[8]$ & $-0.192 \pm 0.009$ & $0.062 \pm 0.009$ & $-0.348 \pm 0.006$ & $0.062 \pm 0.006$ \\
\hline
\end{tabular}

${ }^{a}$ First most positive redox wave. ${ }^{b}$ Second most positive redox wave. ${ }^{c}$ Midpoint between reduction and oxidation peak potentials with respect to $E(\mathrm{Ag} / \mathrm{AgCl})$ at $0.05 \mathrm{~V} \mathrm{~s}^{-1}$ in a deaerated $0.075 \mathrm{M}$ phosphate buffer $(\mathrm{pH} 7)$.

Table 5.1 Voltammetric features for 2, 3, and MV alone and in the presence of cucurbiturils as shown in Figure 5.1.

Figure 5.9 shows the voltammetric scan rate dependence of 2/CB[8]. At a scan rate of 10 $\mathrm{mV} / \mathrm{s}$, the two reversible redox waves described above were distinctly visible. At $100 \mathrm{mV} / \mathrm{s}$, the first reduction wave was at a slightly lower current compared to the baseline, though the corresponding oxidation wave was unchanged. At $1 \mathrm{~V} / \mathrm{s}$, the first reduction wave was essentially nonexistent, and the corresponding oxidation wave was still unchanged. These data suggest that a slow chemical step precedes the reduction of $\mathbf{2}^{\mathbf{4}} / \mathrm{CB}[8]$ to $\mathbf{2}^{\mathbf{3}} / \mathrm{CB}[8]$. At faster scan rates the chemical step is outpaced and reduction directly to $\mathbf{2}^{\mathbf{2}} / \mathrm{CB}[8]$ occurs. Compound $\mathbf{3}$ in $\mathrm{CB}[8]$ demonstrated a similar scan rate dependence (Figure 5.9). 

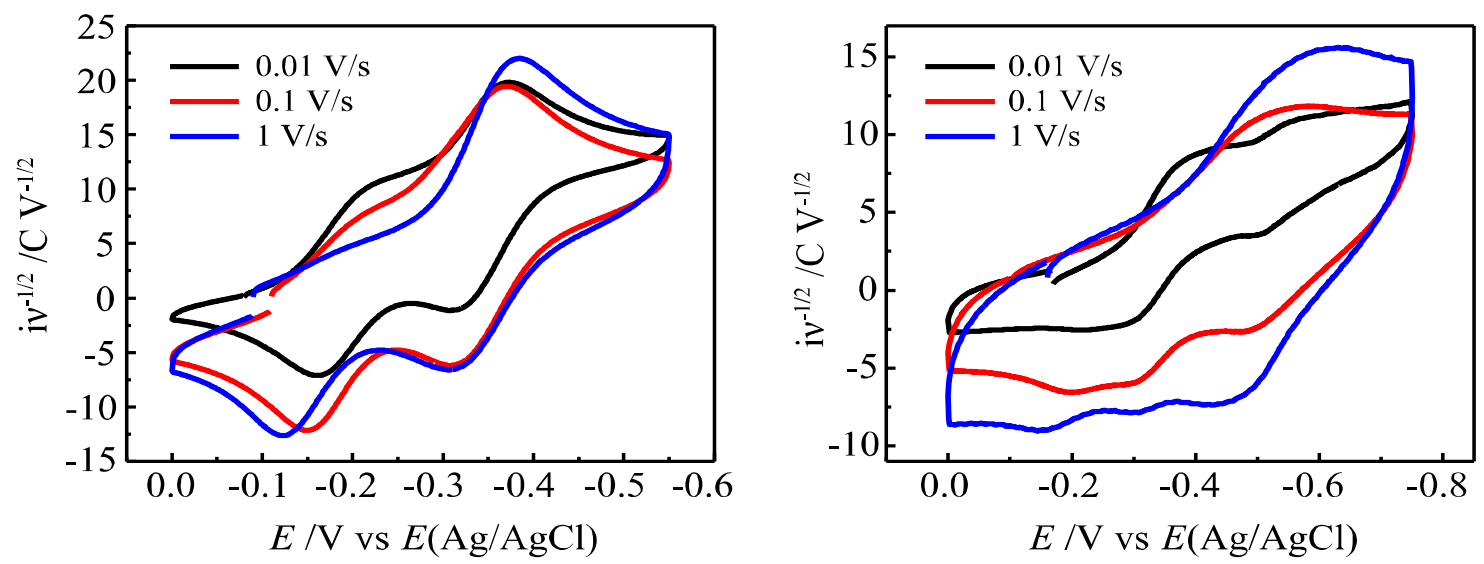

Figure 5.9 Voltammetry of $\mathbf{2}+\mathrm{CB}[8]$ (left) and $\mathbf{3}+\mathrm{CB}[8]$ (right) at various scan rates collected at $0.25 \mathrm{mM}$ viologen with approximately one equivalent of $\mathrm{CB}$ [8]. The electrolyte was deaerated $0.075 \mathrm{M}$ phosphate buffer ( $\mathrm{pH}$ 7). A glassy carbon electrode was used as the working electrode. $\mathrm{Pt}$ mesh was used as the counter electrode and a $\mathrm{Ag}$ wire coated with $\mathrm{AgCl}$ immersed in a fritted compartment containing saturated $\mathrm{KCl}$ was used as the reference electrode.

NMR spectroscopy. ${ }^{1} \mathrm{H}$ NMR spectra were collected to gain further insight on the complexation of $\mathbf{2}$ and $\mathbf{3}$ with $\mathrm{CB}[7]$ and $\mathrm{CB}[8]$. Figure 4 shows the ${ }^{1} \mathrm{H}$ NMR spectra of $\mathbf{2}$ and $\mathbf{3}$ in various oxidation states in the presence and absence of $\mathrm{CB}[7]$ and $\mathrm{CB}[8]$ in $\mathrm{D}_{2} \mathrm{O}$. Upon complexation by $\mathrm{CB}$ [7], all proton signals for $\mathbf{2}^{4+}$ moved to higher chemical shift values (Figure 5.10). The aromatic protons of the viologen units and the bridging methylene peaks were shifted most extensively whereas the peak corresponding to the methyl protons was affected only minimally. The presence of $\mathrm{CB}[8]$ in solution caused qualitatively similar changes in the ${ }^{1} \mathrm{HNMR}$ spectrum of $2^{4+}$. These changes were consistent with the premise that in the 4+ oxidation state, the viologen rings are spread apart in a "linear" dimer conformation due to charge repulsion and the benzyl linker of $\mathbf{2}^{4+}$ spends significant time inside of the cucurbituril cavity. Conversely, the terminal methyl groups on the viologen units are predominantly outside the cavities of the cucurbiturils since there is no possibility of hydrogen bonding by those hydrogens with the $\mathrm{C}=\mathrm{O}$ groups. ${ }^{304}$ The paramagnetic character of $\mathbf{2}^{2+}$ after reduction convoluted the proton signals of the viologens in the corresponding ${ }^{1} \mathrm{H}$ NMR spectra, precluding definitive assignment of viologen orientation upon reduction. However, the significant broadening of the ${ }^{1} \mathrm{H}$ NMR signals from $\mathrm{CB}[8]$ in the presence of reduced dimer was consistent with tight association, and therefore close 


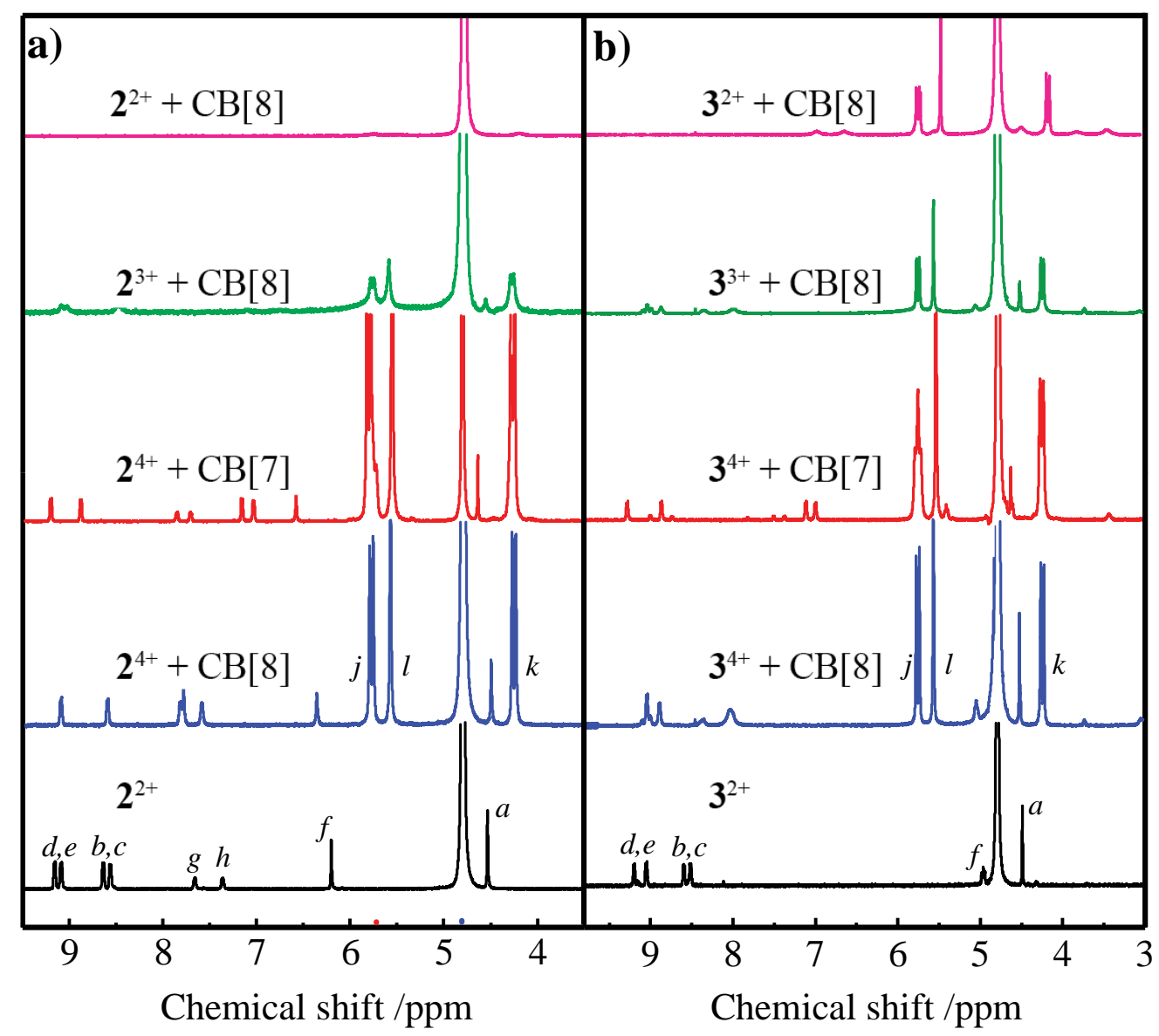

Figure 5.10 ${ }^{1} \mathrm{H}$ NMR spectra of a) aqueous $\mathrm{D}_{2} \mathrm{O}$ solutions of 2 in various oxidation states and in the presence of cucurbiturils and b) aqueous $\mathrm{D}_{2} \mathrm{O}$ solutions of $\mathbf{3}$ in various oxidation states and in the presence of cucurbiturils. Peaks labeled $j, k$, and $l$ correspond to cucurbituril protons.

proximity, of folded $3+$ and 2+ paramagnetic radical dimers within $\mathrm{CB}[8]$. The 2+ dimer species showed a more significant effect on the $\mathrm{CB}$ [8] protons, implying an average closer proximity of radical character to the $\mathrm{CB}[8]$ protons. In Figure $4 \mathrm{~b}$, the spectra for $\mathbf{3}^{4+}$ with and without either $\mathrm{CB}[7]$ or $\mathrm{CB}[8]$ are shown. The changes in the ${ }^{1} \mathrm{H}$ NMR spectral features were generally similar to those in Figure 4a. The signals for the aromatic peaks of the viologen units and the methylene peaks of the propyl linker were more strongly affected by the presence of cucurbituril than the corresponding signatures of the methyl protons. These data suggest that similar to $2^{4+}$, the viologen units and propyl linker of $\mathbf{3}^{4+}$ in the oxidation state are primarily inside the cucurbituril cavities upon complexation. 


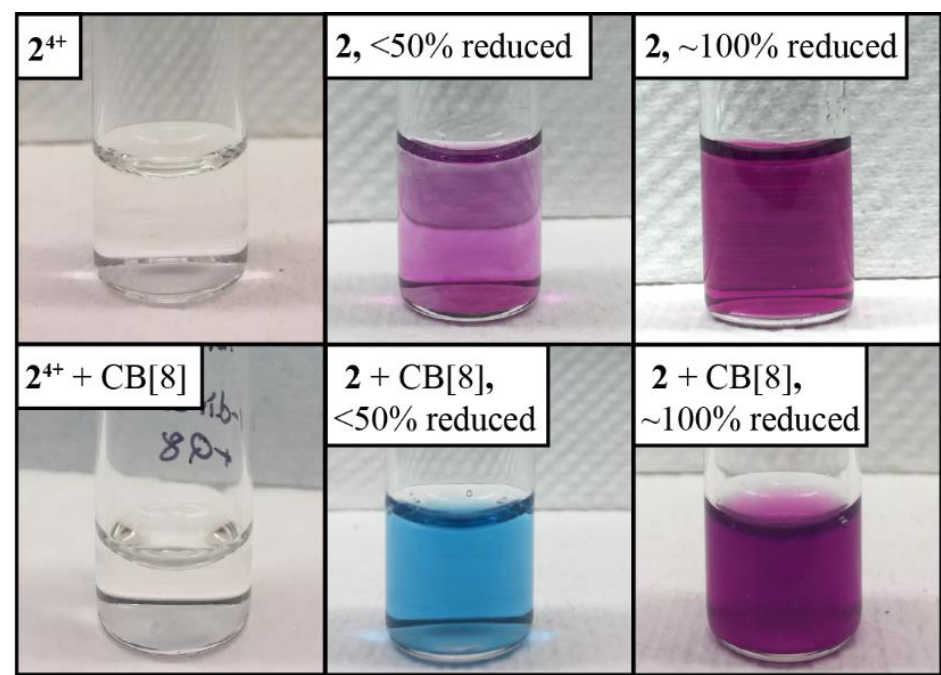

Figure 5.11 Color variation in solutions of 2 with and without CB[8] after addition of reducing equivalents, defined as one reducing equivalent would reduce each viologen unity by $1 \mathrm{e}^{-}$. Top row: $2^{4+}$ (left); 2 after reduction with approximately half of the electrons necessary to fully reduce the solution to $\mathbf{2}^{2+}$ (middle); $\mathbf{2}$ after the solution has been fully reduced to $\mathbf{2}^{2+}$ (right). Bottom row: $\mathbf{2}^{4+}$ with $\mathrm{CB}$ [8] (left); 2 with $\mathrm{CB}$ [8] after reduction with approximately half of the electrons necessary to fully reduce the solution to $2^{2+}$ with $\mathrm{CB}$ [8] (middle); 2 with $\mathrm{CB}[8]$ after the solution has been fully reduced to $2^{2+} / \mathrm{CB}[8]$ (right). The solutions were $0.1 \mathrm{mM} 2$ in water. $\mathrm{CB}$ [8] was added in approximately 1 equivalent.

$U V$-visible-NIR spectroscopy. Figure 5.11 displays photographs of 2 at various stages of reduction with and without $\mathrm{CB}[8]$. In the top left and bottom left are solutions of $0.1 \mathrm{mM} 2^{4+}$ and $0.1 \mathrm{mM}^{4+} / \mathrm{CB}[8]$. Both are colorless. The solutions in the top middle and bottom middle photos were obtained by adding slightly less than half an equivalent of sodium dithionite to the respective solutions. Based on the voltammetry data described above, this addition reduced half of the top solution to $\mathbf{2}^{2+}$, whereas all of the solution in the bottom photo was $\mathbf{2}^{3+} / \mathrm{CB}[8]$. The color of the solution in the top middle was pale purple. The color of the solution in the bottom photo was distinctly blue. The top left and bottom left solutions were obtained by addition of an excess of dithionite to the respective solutions. Both solutions were the same purple color. The top left solution was $2^{2+}$ and the bottom left solution was $2^{2+} / \mathrm{CB}[8]$. The purple color seen in these photos was attributed to the $\mathbf{2}^{2+}$ oxidation state, and the blue color that was only observed in the presence of $\mathrm{CB}[8]$ was attributed to the $\mathbf{2}^{3+}$ oxidation state. It should be noted that these colors were also observed during electrochemical reduction of these solutions. When a solution of $\mathbf{2}^{4+}$ was electrochemically reduced, the color purple was observed to slowly grow in and get steadily darker until the solution was fully reduced to $\mathbf{2}^{2+}$. In contrast, when a solution of $\mathbf{2}^{4+} / \mathrm{CB}[8]$ was 
electrochemically reduced, a blue color was observed, which grew stronger until about half of the charge needed to fully reduce the solution to $2^{2+} / \mathrm{CB}[8]$ had been passed. At this point the color began to change to purple and by the time the solution had been fully reduced, it was the same color as a solution of $\mathbf{2}^{2+}$. These same results were observed with $\mathbf{3}^{4+}$ and $\mathrm{CB}[8]$.

Figure 5.12 shows the UV-vis-NIR spectra of each set of compounds. The NIR spectral region was cut off at $1400 \mathrm{~nm}$ due to strong absorption by water at longer wavelengths. Both $\mathbf{2}^{2+}$ and $3^{2+}$ had broad asymmetric absorbances between 500 and $600 \mathrm{~nm}$ and a second broad band around $850 \mathrm{~nm}$, characteristic of viologen pimers in water. ${ }^{296,302,305-306}$ Addition of CB[7] caused little change to the spectrum of either compound. Addition of $\mathrm{CB}[8]$ also produced little change to the spectra when the viologens were fully reduced to the $2+$ states. However, when the viologens were partially reduced to the $3+$ states in $\mathrm{CB}[8]$, dramatic changes to the spectra were observed. In this case, the visible band was shifted from 536 to $623 \mathrm{~nm}$ for 2 and from 534 to $622 \mathrm{~nm}$ for 3 . The NIR band disappeared completely from the spectrum of $\mathbf{2}$, and in the spectrum of $\mathbf{3}$ it shifted from about $841 \mathrm{~nm}$ into the cut-off region, i.e. $>1400 \mathrm{~nm}$. These features are characteristic of viologen dimers in the $3+$ oxidation state. ${ }^{298,301}$

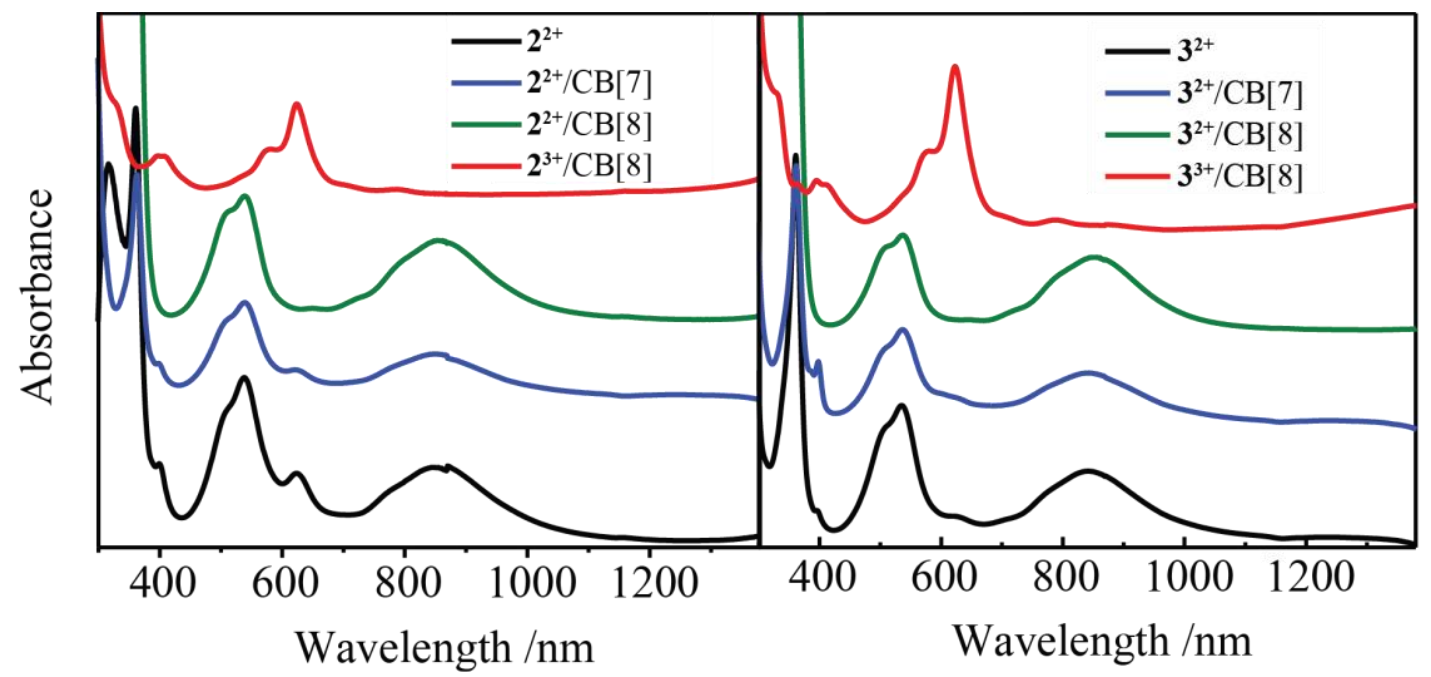

Figure 5.12 Absorbance spectra of 2 and 3 with and without cucurbiturils in solution after reduction to the $2+$ or $3+$ states. (left) $\mathbf{2}^{2+}$ (black), $\mathbf{2}^{2+}$ in the presence of an equivalent of $\mathrm{CB}$ [7] (blue), $\mathbf{2}^{2+}$ in the presence of an equivalent of $\mathrm{CB}[8]$ (green), and $\mathbf{2}^{3+}$ in the presence of an equivalent of $\mathrm{CB}$ [8] (red). (right) $\mathbf{3}^{2+}$ (black), $\mathbf{3}^{2+}$ in the presence of an equivalent of $\mathrm{CB}$ [7] (blue), $3^{2+}$ in the presence of an equivalent of $\mathrm{CB}[8]$ (green), and $\mathbf{3}^{3+}$ in the presence of an equivalent of $\mathrm{CB}[8]$ (red). 
Lifetime in air. Figure 5.13 demonstrates the relative rates of decay of the reduced viologens upon exposure to oxygen with and without $\mathrm{CB}[8]$ present. In Figure 5.13a, a $30 \mu \mathrm{M}$ solution of $\mathbf{2}^{4+}$ was fully electrolyzed to $\mathbf{2}^{2+}$ under a stream of nitrogen. Upon fully reducing the solution, the nitrogen stream and electrodes were removed from the cell and immediately the UVvis absorbance of the solution was monitored by taking a spectrum every 15 seconds. Figure 5.13b shows the same experiment done using a $30 \mu \mathrm{M}$ solution of $2^{4+}$ in the presence of one equivalent of $\mathrm{CB}[8]$. In Figure 5.13a, the absorbance at the $\lambda_{\max }(536 \mathrm{~nm})$ decayed over time, resulting finally in a featureless spectrum. In Figure 5.13b, the peak at $536 \mathrm{~nm}$ decayed and the peak at $623 \mathrm{~nm}$ grew in with an isosbestic point, until the peak at $623 \mathrm{~nm}$ began to decay as well. The final spectrum in this case was also featureless.

In Figure 5.13c, the black squares indicate the absorbance at $536 \mathrm{~nm}$ of the spectrum in Figure 5.13a, which was assigned to the species $2^{2+}$, and in red is the absorbance at $623 \mathrm{~nm}$ in Figure 5.13b, which was assigned to the species $2^{3+} / \mathrm{CB}[8]$. The absorbance at $536 \mathrm{~nm}$ reached zero within $90 \mathrm{~s}$, but the absorbance at $623 \mathrm{~nm}$ decayed much more slowly, reaching zero after around 15 minutes. Each of these decay plots in Figure $5.13 \mathrm{c}$ were fitted with an exponential decay function. The time constant for decay of $\mathbf{2}^{2+}$ without $\mathrm{CB}[8]$ was $41 \pm 2 \mathrm{~s}$, and the half-life was 28 $\pm 1 \mathrm{~s}$. The time constant for decay of $\mathbf{2}^{3+}$ in the presence of $\mathrm{CB}[8]$ (taking the maximum absorbance value in the plot in Figure 5.13c as the starting value) was $206 \pm 1 \mathrm{~s}$ and the half-life was $143 \pm 1 \mathrm{~s}$.

Figures 5.13d-f demonstrate a similar effect with compound 3. In the absence of CB[8], the decay time constant was $16 \pm 1 \mathrm{~s}$ and the half-life was $11 \pm 1 \mathrm{~s}$. In the presence of $\mathrm{CB}$ [8], the decay time constant was $126 \pm 5 \mathrm{~s}$ and the half-life was $87 \pm 3 \mathrm{~s}$.

Figures 5.14g-i show the same experiment for MV. In the absence of $\mathrm{CB}$ [8], the decay time constant was $21 \pm 1 \mathrm{~s}$ and the half-life was $15 \pm 1 \mathrm{~s}$. In the presence of $\mathrm{CB}$ [8], the decay time constant was $45 \pm 2 \mathrm{~s}$ and the half-life was $31 \pm 1 \mathrm{~s}$.

$\mathrm{R}^{2}$ values for the exponential decay fits were 0.951 for $\mathbf{2}^{2+} ; 0.999$ for $\mathbf{2}^{3+} / \mathrm{CB}[8] ; 0.950$ for $\mathbf{3}^{2+} ; 0.968$ for $\mathbf{3}^{3+} / \mathrm{CB}[8]$; 0.942 for $\mathbf{M V}^{1+} ;$ and 0.953 for $\mathbf{M V}^{1+} / \mathrm{CB}[8]$. 

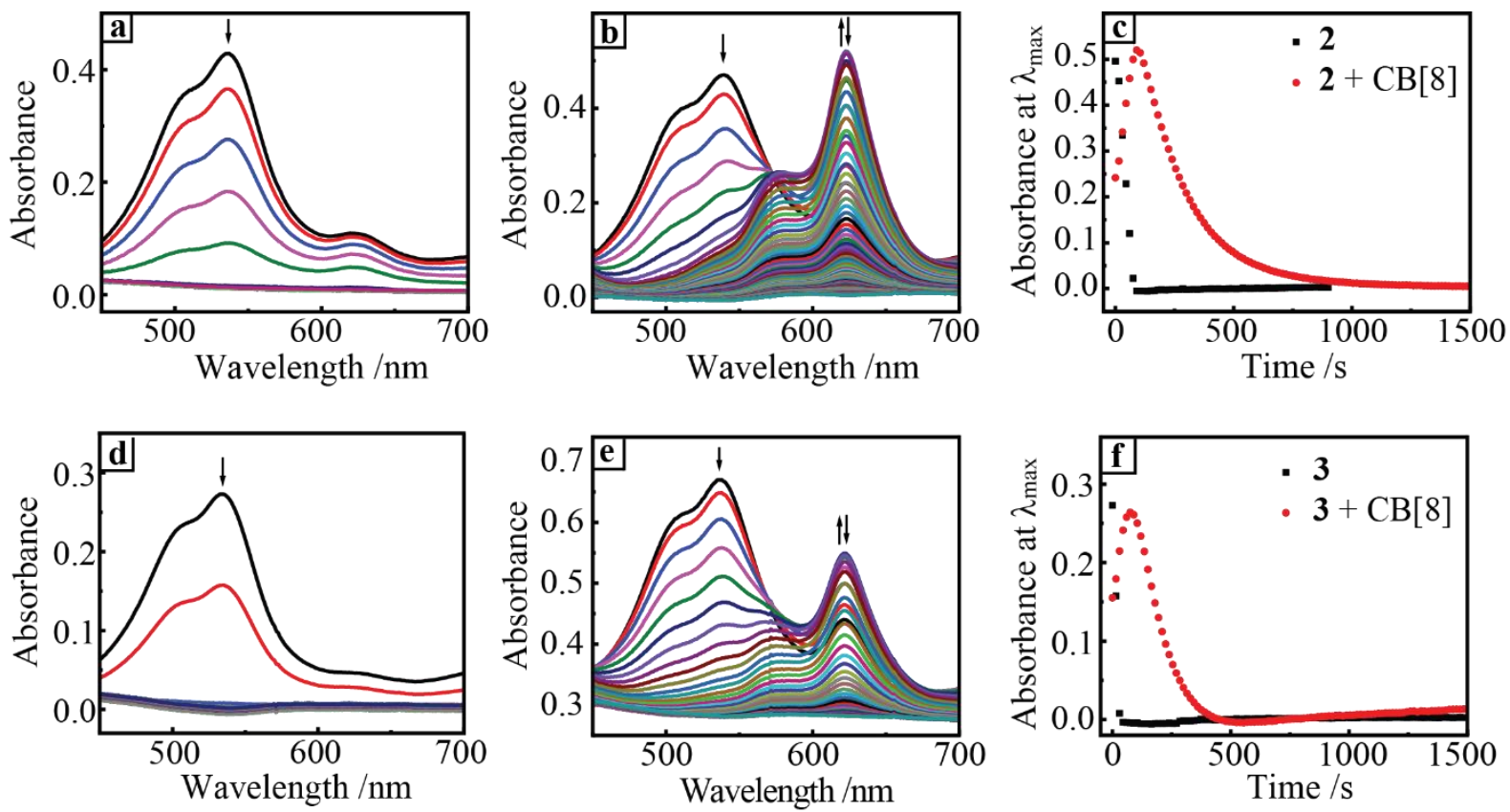

Figure 5.13 a) Time-dependent absorbance spectra for $2^{2+}$ in aqueous solution recorded at $15 \mathrm{~s}$ intervals over $25 \mathrm{~min}$. b) Time-dependent absorbance spectra for $\mathbf{2}^{2+}$ in same solution but with one equivalent of $\mathrm{CB}$ [8]. c) Time-dependence of the absorbance maxima in Figure 5.13a at $\lambda=536$ $\mathrm{nm}$ and in Figure 5.13b at $\lambda=623 \mathrm{~nm}$. d) Time-dependent absorbance spectra for $3^{2+}$ in aqueous solution. e) Time-dependent absorbance spectra for $\mathbf{3}^{2+}$ in the same solution but with one equivalent of CB[8]. f) Time-dependence of the absorbance maxima in Figure 5.13d at $\lambda=534 \mathrm{~nm}$ and in Figure 5.13e at $\lambda=622 \mathrm{~nm}$.
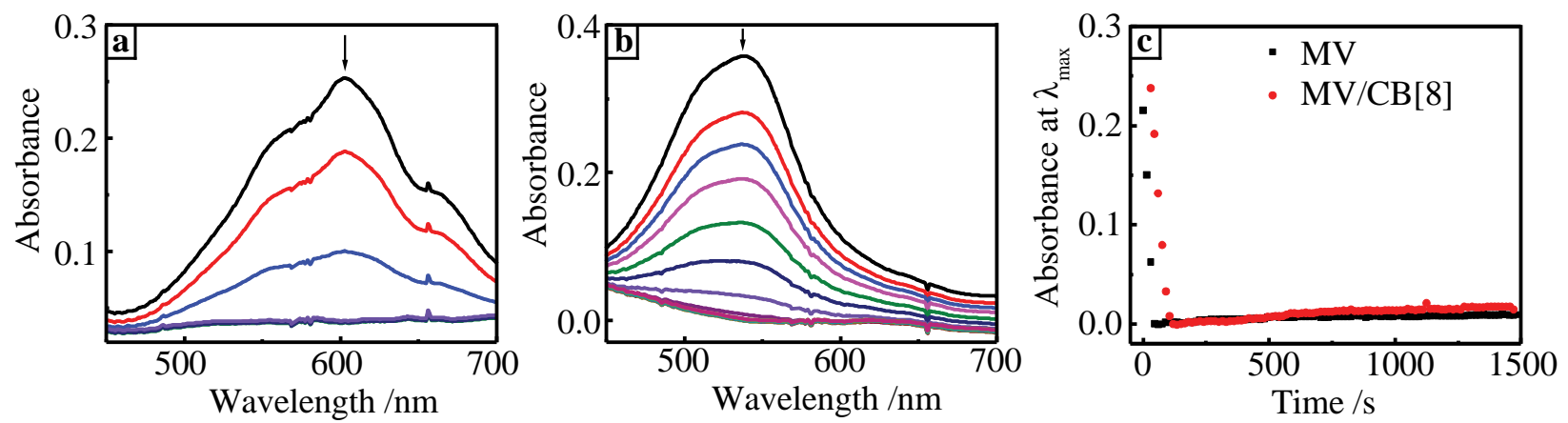

Figure 5.14 a.) Time-dependent absorption spectra for $\mathbf{M V}^{\cdot+}$ in phosphate buffer (pH7) recorded at $15 \mathrm{~s}$ intervals over $25 \mathrm{~min}$. b) Time-dependent absorption spectra for $\mathbf{M V}^{{ }^{+}}$in same solution but with one equivalent of $\mathrm{CB}[8]$. c) Time-dependence of the absorption maxima in Figure 5.14a at $\lambda$ $=603 \mathrm{~nm}$ and in Figure 5.14b at $\lambda=538 \mathrm{~nm}$. 


\subsection{Discussion.}

The data presented here demonstrate the formation of an unusually stable radical viologen species through supramolecular complexation in solution that is disctinct from previously explored viologen radical pimers. This species, a monoradical bis-viologen, has never previously been observed, but similar species have been observed in the case of the "blue-box molecule"296, 301, 307309 (Figure 5.18) and in non-aqueous solvents. ${ }^{298}$ This is the first time that a simple bis-viologen monoradical species has been isolated through supramolecular assembly. It is also the first time that a viologen radical species has been shown to be stabilized toward reaction with dissolved oxygen. This is further discussed below for the case of compound 2. Compound $\mathbf{3}$ demonstrated similar results as compound $\mathbf{2}$.

Intra- and Intermolecular Factors Necessary to Form Stabilized, Reduced Viologen Dimer. Singly reduced viologens can form pairs, often called pimers, in solution with strong overlap of the $\pi$ orbitals between the two units. ${ }^{296,298,302,310}$ The formation of these pimers can have large association constants $\left(\sim 10^{2} \mathrm{M}^{-1}\right)^{253}$ since the $\pi-\pi$ stacking allows for stabilization of the individual viologen radicals. ${ }^{299}, 311$ Studies have shown that this stabilization is mostly attributable to dispersion and delocalization of the two SOMO orbitals across all surfaces of the stacked viologen moieties. ${ }^{299,312-314}$ Generally, such interactions have been described as pancake bonding. ${ }^{299,311-314}$ These delocalized bis-radical species form readily when viologens are reduced in sufficiently high concentrations ${ }^{305,315}$ or when reduced viologen units are in forced close proximity. ${ }^{296}$ The latter scenario is clearly evidenced in the voltammetry of free $2^{4+}$ and $3^{4+}$ by the notable positive shift of the voltammetric response for the reduction from the $4+$ to $2+$ states compared to the reduction of $\mathbf{M V}^{2+}$ to $\mathbf{M V}^{\bullet+}$. The greater positive shift in redox potential as well as the clearer voltammetry observed with $\mathbf{2}$ as compared to 3 suggest that the preorganization of direct $\pi-\pi$ overlap observed in modeling the rigid ortho-benzyl linkage increases the likelihood of stabilized $\pi$ - $\pi$ stacking between radical viologen units of $\mathbf{2}^{2+}$. The equilibrium geometry and flexibility of the propyl linker provides comparatively less probability that the two reduced viologen units of $\mathbf{3}^{2+}$ are well positioned for delocalization of the bis-radical. The voltammetry, NMR, and absorbance data for $\mathbf{2}^{2+}$ and $\mathbf{3}^{2+}$ are in line with the previous literature on $\pi$-stacked bis-radical pimers. ${ }^{253,296,305,315}$

In the presence of cucurbituril complexing agents, the reductions of $\mathbf{2}$ and $\mathbf{3}$ show evidence for reduced viologen radicals unlike the previously described $\pi$-stacked bis-radical pimers. Observations in the presence of $\mathrm{CB}[7]$ show behavior similar to that in previous reports of viologen 
complexation with $\mathrm{CB}[7]$; that is, a negative shift in the reduction potential and little change in the absorbance spectrum. ${ }^{272,293}$ This is attributed to the small size of the CB[7] cavity, which is not able to accommodate two stacked aromatic moieties. ${ }^{272,291,293} \mathrm{CB}[8]$, on the other hand, is uniquely suited to host two reduced, tethered, and stacked viologen units simultaneously because of its large cavity size. $\mathrm{CB}[8]$ thereby promotes the formation of a new type of stabilized radical pimer. The existence of this new radical pimer is seen in the splitting of the voltammetric wave of 2 in the presence of $\mathrm{CB}[8]$ and in the blue color and unique absorbance spectrum of partially reduced 2 in the presence of $\mathrm{CB}[8]$. This effect is also seen the voltammetry and absorbance spectrum of $\mathbf{3}$. In contrast, the inclusion of two reduced $\mathbf{M V}^{+}$equivalents in $\mathrm{CB}$ [8] show no evidence of a stable 3+ radical pimer or otherwise remarkable properties. Previously reported reduction of a viologen dimer connected with a longer flexible linker (hexyl) in the presence of $\mathrm{CB}[8]$ also showed no evidence of a stable $3+$ radical state. ${ }^{263}$ Accordingly, the data here are clear that both dimer pre-organization and complexation within a sufficiently large cavity are necessary to make the blue viologen dimer in the $3+$ oxidation state.

A scheme showing the elementary electrochemical and chemical steps describing the formation of the monoradical for 2 in the presence of $\mathrm{CB}[8]$ is proposed in Figure 5.15. Presumably, the formation of the corresponding monoradical of $\mathbf{3}^{3+}$ is similar, albeit with additional competing equilibria for conformations that are not productive towards the $3+$ state. In this figure, vertical transitions denote chemical steps constituting either conformation or complexation changes while horizontal transitions represent $1 \mathrm{e}^{-}$electrochemical charge transfers. Based on the collected voltammetry and calculations of conformer stability (Figures 5.5, 5.6, and 5.8), the equilibrium form of $\mathbf{2}^{4+}$ has the viologen units spread far apart, i.e. as a linear conformer with $K_{2}<<1$. This linear conformation of $2^{4+}$ binds in a 1:1 ratio with CB[8] and the complex has a disassociation constant $K_{1}=8.3 \times 10^{-7} \mathrm{M}$ as measured by ITC (Figure 5.7). The reduction of both viologen units on unbound $\mathbf{2}$ are degenerate, i.e. the standard potentials are equivalent and the reduction processes are independent of each other, as evidenced by the narrow $\Delta E_{p}$ in the voltammetry of $\mathbf{2}^{4+}$ alone. ${ }^{303}$ Based on the shift of the voltammetric wave of $\mathbf{2}^{4+}$ relative to the voltammetric wave of $\mathbf{M V}^{2+}$, the value of $K_{3} \gg$, i.e. $\mathbf{2}^{2+}$ changes conformation to stabilize the viologen units by co-facial pairing. In the presence of $\mathrm{CB}[8]$, we infer the paired form of $\mathbf{2}^{2+}$ partitions readily into $\mathrm{CB}[8]$ with $K_{4}>K_{1}$ since the voltammetric wave at relatively fast scan rates $\left(>1 \mathrm{~V} \mathrm{~s}^{-1}\right.$ ) for the $4+/ 2+$ condition is shifted towards an even more positive potential (Figure 5.8). 


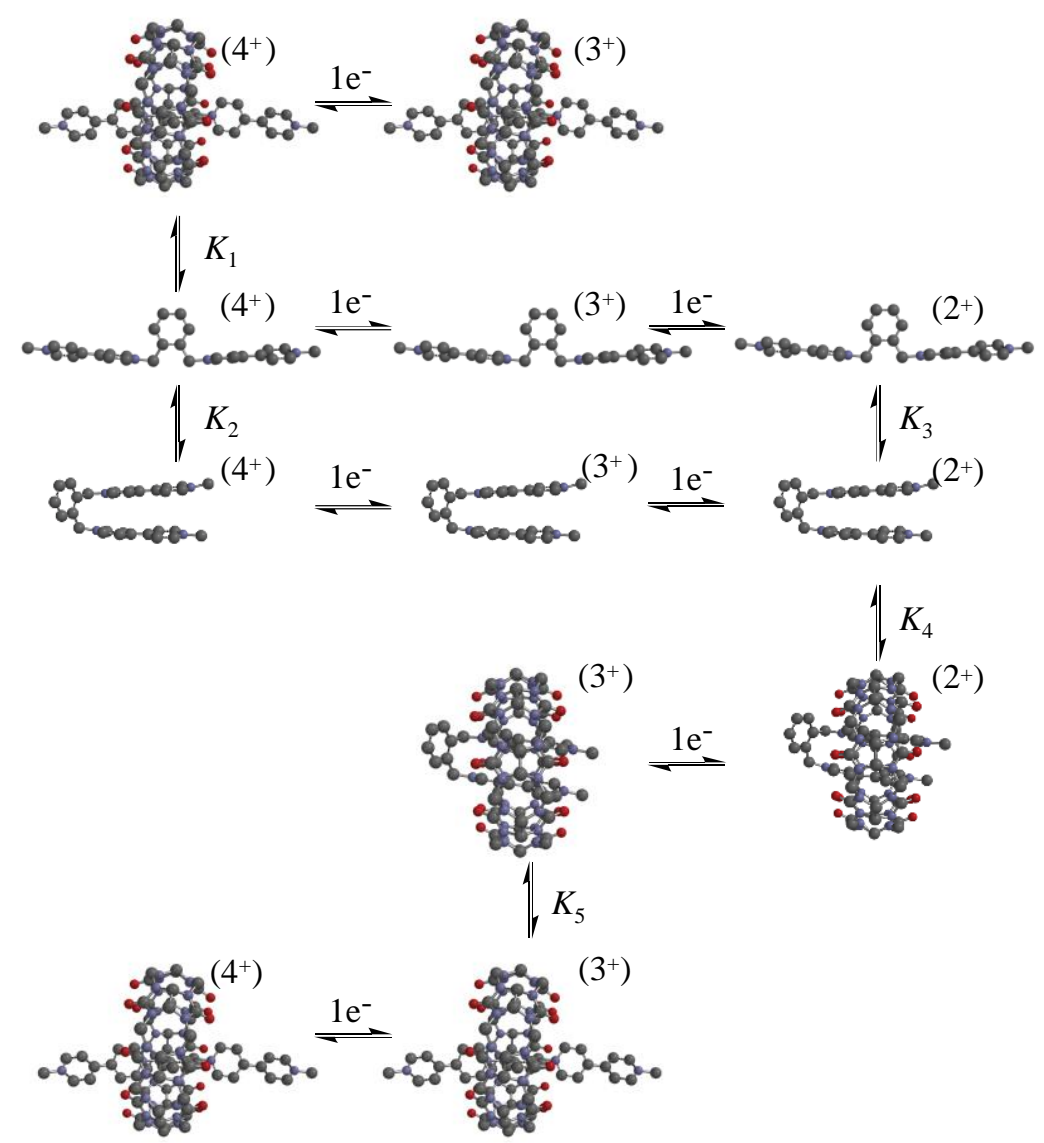

Figure 5.15 Proposed elementary chemical and electrochemical steps involved in the reduction of 2 in the presence of $\mathrm{CB}[8]$.

Reduction of $2^{4+}$ in the presence of $\mathrm{CB}[8]$ at slower scan rates $\left(<0.5 \mathrm{Vs}^{-1}\right)$ allows access to the significantly stabilized $4+/ 3+$ transition, for which the folded $\mathbf{2}^{3+}$ is likely very tightly associated with $\mathrm{CB}[8]$, combining to a remarkable positive shit in the 1e- reduction wave. The large value of $K_{4}$ is also responsible for the scan rate independence of oxidation observed for $\mathbf{2}^{2+}$ in the presence of $\mathrm{CB}[8]$. The preorganized structure requires no conformational rearrangement to access the stable $2^{3+}$ complex, and so sequential $2+/ 3+$ and $3+/ 4+$ oxidation waves are observed at all scan rates.

Electronic Structure of Reduced, Viologen Monoradical The presented absorbance spectra clearly indicate that the electronic structure of the $3+$ state is very different than that of the bisradical form or even of a singly reduced methyl viologen in organic solvents. ${ }^{296,306}$ To gain insight into this empirical observation, molecular orbital calculations were performed on energy minimized structures of $\mathbf{2}^{3+}$ and $\mathbf{2}^{2+}$ encapsulated in CB[8]. Figure 5.16 shows computations of the energetics for the $3+$ radical form of 2 relative to the $2+$ bis-radical. In free solution both species 
yield equilibrium geometries favoring a partially-folded state that balances charge-repulsion with radical dispersion forces. For $\mathbf{2}$, this geometry leads to minimal overlap and perturbation of the $\mathbf{2}^{3+}$ singlet highest occupied molecular orbital (sHOMO) and predicts electrochemical neardegeneracy of the $2^{\text {nd }}$ reduction to the $2+$ state (Figure 5.16). In the presence of $\mathrm{CB}$ [8], both reduced species show strong complexation inside the host cavity. The resultant equilibrium geometry of the dimer is completely folded with a $\pi-\pi$ stacking distance $<3.6 \AA$. In this conformation within $\mathrm{CB}[8]$, the sHOMO of $\mathbf{2}^{3+}$ shows a strong and stabilizing perturbation due to delocalization of the radical across both viologen moieties, with perfectly symmetric constructive overlap at the $\pi-\pi$ interface (Figure 5.16). This constructive (bonding) symmetry suggests a significant stabilization of the complex between $\mathbf{2}^{3+}$ and $\mathrm{CB}$ [8], with a majority of the $\mathbf{2}^{3+}$ sHOMO orbital density existing between the aromatic groups, effectively burying the radical between the two viologen units. Essentially an identical sHOMO orbital was calculated for energy-minimized $\mathbf{3}^{3+}$ in $\mathrm{CB}$ [8] (Figure 5.16).

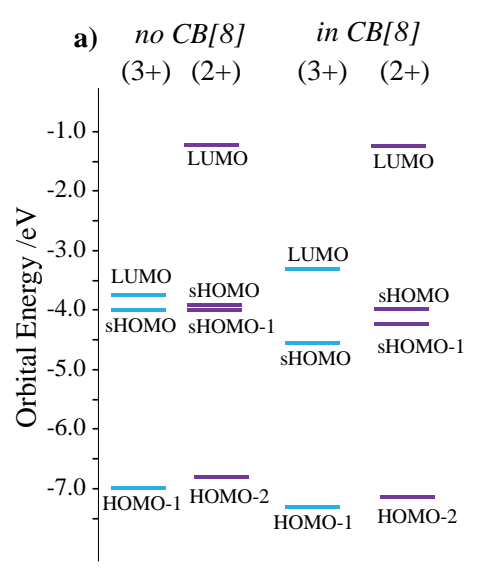

b) sHOMO orbitals
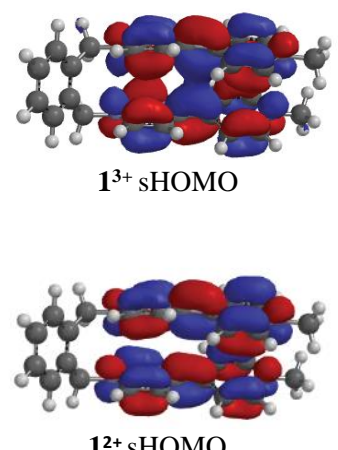

$\mathbf{1}^{2+} \mathrm{sHOMO}$ c) $\mathbf{1}^{3+}$ in $\mathrm{CB}[8]$

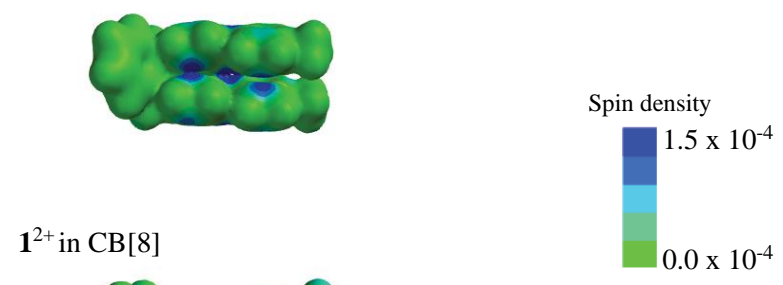

Figure 5.16 a) The relative energies of molecular orbitals from DFT calculations (Spartan 16,B97D, 6-311+G*) showing the $3+$ and $2+$ states of 2 both without and within $\mathrm{CB}[8] . \mathrm{b}$ ) Graphical depiction of the LUMO of $\mathbf{2}$ in the $3+$ oxidation state and the sHOMO of $\mathbf{2}$ in the 2+ oxidation state. c) The spin densities of 2 inside $\mathrm{CB}[8]$ in the $3+$ and $2+$ oxidation states are shown, with $\mathrm{CB}[8]$ omitted for clarity. Side and top views of the $\mathbf{2}$ are shown, with the blue regions highlighting the regions where the radical density is highest.

The spin density map of the complex between $\mathbf{2}^{3+}$ and $\mathrm{CB}[8]$ further supports the point that the transferrable electron is not readily accessible. The density of unpaired spin is mostly located on the internal $\pi$-surface of the folded dimer (Figure 5.16). Conversely, the sHOMO of the $\mathbf{1}^{2+}$ 
dimer in $\mathrm{CB}[8]$ has a node running between the two viologen units at the $\pi-\pi$ interface (Figure 5.16). This aspect can also be visualized in the corresponding spin density map of the complex between $2^{2+}$ bis-radical and $\mathrm{CB}[8]$ which shows significant unpaired spin density on both the internal lower energy singlet HOMO-1 (sHOMO-1) and external (sHOMO) viologen surfaces. These characteristics are further consistent with the "pancake bonding" concept. ${ }^{316-319}$ We posit that the uniform distribution of electron density on both surfaces of the viologens for the sHOMO orbital of $\mathbf{2}^{2+}$ (and $\mathbf{3}^{2+}$ ) results in good electronic coupling with oxidants and corresponding fast electron transfer.

Stability in the Presence of $\mathrm{O}_{2}$ Based on the preceding discussion, we propose that the resultant stability of $\mathbf{2}^{3+}$ and $\mathbf{3}^{3+}$ complexed with $\mathrm{CB}[8]$ in air is largely a consequence of slower charge-transfer kinetics. The observed stability against oxidation of these species by $\mathrm{O}_{2}$ is in accord with their proposed electronic structures. Since the CB[8] cavities force the viologen units in close proximity to form a stable pocket where the radical electron resides between the two aromatic units, this structure inhibits electronic coupling with external oxidants. In effect, this transferrable electron becomes significantly less accessible to oxidants like $\mathrm{O}_{2}$ and hence the rate of oxidation of the viologen dimer back to the 4+ state is slowed. In effect, the viologen rings represent tunneling barriers that inhibit transfer of that specific electron. For oxidation of the $2+$ state, the viologen rings do not act as tunneling barriers since the electron density is explicitly located on their exterior as well as interior. As a result, $\mathrm{O}_{2}$ can readily oxidize the $2+$ state even when the dimer resides inside of $\mathrm{CB}$ [8]. A further contrast along these lines is clear when considering the spin density map for the energy-minimized conformation of two $\mathbf{M V}^{+}$in $\mathrm{CB}$ [8]. In this case, the pimer adopts a twisted conformation (Figure 5.17).

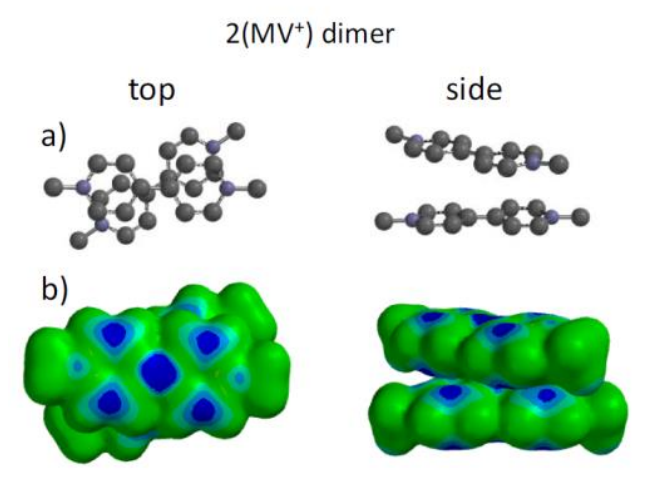

Figure 5.17 Energy minimized structure (hydrogens not shown) (a) and spin density map (b) of the $2\left(\mathbf{M V}^{+}\right)$pimer showing the known twisted conformation of the viologen moieties. 
Relation to Other Viologen Materials Although this report is the first explicit use of preorganization and complexation principles to stabilize a reduced viologen, there are two examples that have some parallels. The first area is redox active polymers based on viologen moieties. ${ }^{257-259}$ In the context of charge-storing catholytes, an organic polymer with pendant side chains consisting of the ortho-benzyl viologen dimers has been reported. ${ }^{259}$ Unlike other poly-viologens, this particular polymer featured a reduced form that was unusually stable in air. The origins of this stability were not explored since that particular material was not easy to oxidize back electrochemically and therefore not deemed readily useful for rapid charge/discharge cycling in redox flow batteries. The oxidation state of this unusually stable viologen was the fully reduced species, i.e. each viologen dimer in the polymer was a $2+$ bis-radical species. Thus, the stabilization effects were not the same as the stabilization of the $3+$ viologen monoradicals described here. Nevertheless, it is possible that the polymeric structure afforded an environment that forced the viologen dimers into a stabilized conformation, similar to the synergistic effects between preorganization and complexation seen here.

Similarly, dually tethered viologen dimers with the proper structure might also enable the $3+$ monoradical state. Specifically, cyclophane compounds can have similar structures as $\mathbf{2}^{3+}$ complexed with $\mathrm{CB}[8]$, but this is because both ends are linked by phenyl groups rather than because of complexation. The most famous cyclophane is cyclobis(paraquat-p-phenylene), i.e. "blue-box" (Figure 5.18), and is widely employed in catenanes and rotaxanes. ${ }^{307,320-321}$ The space between viologen units in para-"blue-box" compounds is large enough to accommodate guest molecules but conversely precludes the possibility of forming a tight pocket to stabilize the $3+$ monoradical. Accordingly, these compounds have never shown a stabilized reduced state. The ortho form of this cyclophane is less studied. The small space between viologen units precludes molecular guests and this molecule is exceedingly difficult to synthesize with high yields. Its synthesis requires inordinately long reaction times because of solubility incompatibilities of the precursors and low concentrations to prevent polymerization pathways. ${ }^{309,322}$ The available data on the electrochemical properties of this compound are scarce, with only a few reports showing some optical data upon reduction or photoexcitation. ${ }^{310,323-325}$ Still, these scant data are in accord with the data shown here and suggest the electrochemical properties (and stability in air) of ortho"blue-box" could be similar. Experimental verification of this hypothesis would prove insightful. 
However, the larger take away of this work is that the combination of intramolecular preorganization and complexation of much simpler-to-synthesize viologens circumvents the need to prepare such cyclophanes. More work is needed to determine whether design improvements can be made to further enhance the stability of reduced viologens.
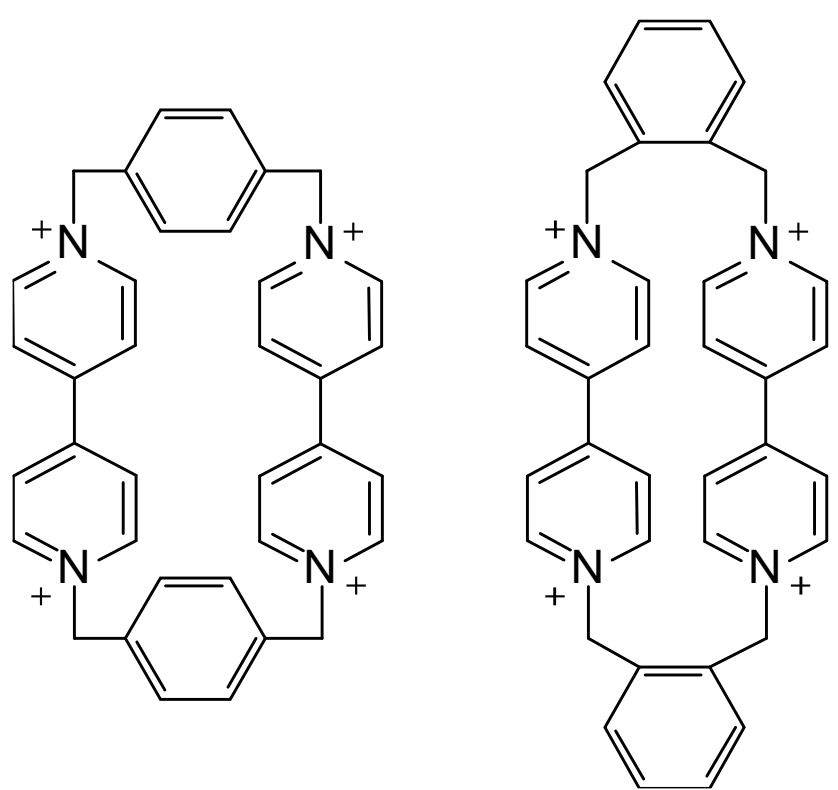

Figure 5.18 Structures of (left) cyclobis(paraquat-p-phenylene) and (right) cyclobis(paraquat-ophenylene).

\subsection{Conclusions.}

This work demonstrates that it is possible to form a stable, reduced viologen radical in aqueous solution through a combination of intramolecular and supramolecular assemblies. The hierarchical reduction of pre-organized viologen moieties and encapsulation of this folded species inside the cavity of the rigid macrocyclic host $\mathrm{CB}[8]$ accesses a conformation that specifically stabilizes a monoradical, $3+$ cation species. This reduced radical possesses a distinct visible light absorbance profile that is unlike any previously reported viologen pimer. Instead, its optical properties bear strong resemblance to bis-viologen cyclophanes with ortho-benzyl linkers. Unlike such cyclophanes which are difficult to synthesize, the compounds shown here can be synthesized in almost quantitative yields and the supramolecular assemblies form readily in water upon electrochemical reduction. These encapsulated monoradicals are significantly more stable in the presence of $\mathrm{O}_{2}$, highlighting a unique reactivity relative to other viologen species. Molecular modeling analyses suggest the stability of this monoradical species results from the central location 
of the SOMO between the viologen rings. Due to the ease of preparation, these monoradical compounds are amenable to further spectroscopic studies that detail their electronic structure. More generally, these results suggest new design motifs for viologens in applications where storing reduced equivalents of charge such as electrochromic windows, redox flow batteries, and solar fuel storage systems is critical. 


\section{Chapter 6. Future Work}

\subsection{Covalent grafting small molecules to RGO thin films}

Molecular catalysts can be immobilized on electrode surfaces through covalent grafting to the surface. ${ }^{34,214-216}$ Covalent grafting is advantageous over physisorption (discussed in Chapter 3) because it is inherently a more robust attachment method (see Introduction). Graphitic materials provide convenient surfaces for grafting small molecules as they provide many functional group handholds. Not only do graphitic carbons contain $\mathrm{C}=\mathrm{C}$ and $\mathrm{C}-\mathrm{H}$ functionalities, but native oxides at defect regions in the form of $\mathrm{C}-\mathrm{OH}, \mathrm{C}-\mathrm{O}-\mathrm{C}$, and $\mathrm{COOH}$ groups also exist on carbon surfaces. This allows for a wide variety of grafting reactions on graphitic carbons. Several examples of covalent attachment of small molecules on graphitic surfaces are given in a review by Campisciano et al. ${ }^{54}$

Described here is preliminary work on three methods for grafting small molecules to RGO thin films. Each of these methods has precedent in the literature, but this is the first time they have been reported for the particular molecules listed below. Each of these methods can be extended to grafting a derivative of the catalyst 1 to RGO films on electrode surfaces. As described below, each grafting method requires the molecule of interest to possess a specific functional group (these are amine, alkoxysilane, and alkyne). Current work is underway to synthesize derivatives of $\mathbf{1}$ that possess one of these functional groups.

Bromoaniline grafting to GO films. The first method of covalent attachment involves amine attack at epoxide groups on the GO surface. This grafting method has been used extensively in published reports, mainly for grafting long alkyl chains to GO to alter its surface chemistry or electronic behavior. ${ }^{55,88-90}$ Few reports exist on the grafting of functional molecules, such as catalysts, to GO surfaces using this method. As a proof-of-concept, in this report 4-bromoaniline was grafted to GO. This molecule was chosen because it was thought to exhibit similar steric preferences and electronics as the ligands of $\mathbf{1}$. The method for grafting via amine functionalities is extremely simple to carry out; however, it requires the GO to be in suspension during the 
reaction. Attempts were made on GO and RGO thin films, but none were successful. Likely steric hindrance becomes a limiting factor for reaction on a film surface.

The reaction was successfully carried out by adding about $50 \mathrm{mg}$ of 4-bromoaniline to about $5 \mathrm{~mL}$ of a suspension of GO in methanol under a nitrogen atmosphere. This mixture was stirred at about $70^{\circ} \mathrm{C}$ and aliquots were removed after various periods of time, washed with clean methanol, and cast as films on degreased Si wafers. Analysis by XPS shows that the reaction reaches approximate completion after only 2 hours (Figure 6.1). Likely the surface coverage can be improved by increasing the number of epoxide groups on GO, which can be accomplished through epoxidation reactions. ${ }^{326}$

Reduction of these bromoaniline-modified GO films to RGO using a solution of cobaltocene or cobaltocene in the presence of $\operatorname{acid}^{327}$ led to loss of the bromine XPS signal. Assumedly the reducing power of cobaltocene was strong enough to reduce the $\mathrm{C}-\mathrm{N}$ bonds of the grafted molecules. To determine whether reduction of GO to RGO with retention of the bromoaniline moiety was possible, electrochemical reductions at varied potentials were attempted. This was carried out by casting films of bromoaniline-modified GO on FTO-coated glass electrodes, immersing these electrodes in a deaerated $1 \mathrm{M} \mathrm{KCl}$ electrolyte containing $1 \mathrm{mM} \mathrm{HCl}$, and cycling three times between the open circuit potential and a potential more negative of that. Four different films were produced in this way, cycling to $-0.75 \mathrm{~V},-0.85 \mathrm{~V},-0.95 \mathrm{~V}$, and $-1.05 \mathrm{~V}$ (vs. $\mathrm{Ag} \mid \mathrm{AgCl}$ ), respectively. Figure 6.2 shows the $\mathrm{Br} 3 \mathrm{~d}$ and $\mathrm{C}$ 1s XP spectra of each film after voltammetric cycling. The $\mathrm{Br} 3 \mathrm{~d}$ signal does not change in intensity at any of the reduction potentials. However, the $\mathrm{C}$ 1s peak shape changes dramatically, becoming narrower and with a less pronounced shoulder at higher binding energy with more negative reduction potentials during $\mathrm{CV}$ cycling. This data demonstrates that cycling to $-1.05 \mathrm{~V}$ in a $1 \mathrm{mM} \mathrm{HCl}$ solution results in a reduced material with a $\mathrm{C} 1$ s peak that displays graphitic character, while retaining the $\mathrm{Br} 3 \mathrm{~d}$ signal. This grafting method, then, is a viable route for covalently immobilizing a derivative of $\mathbf{1}$ to RGO thin films. 


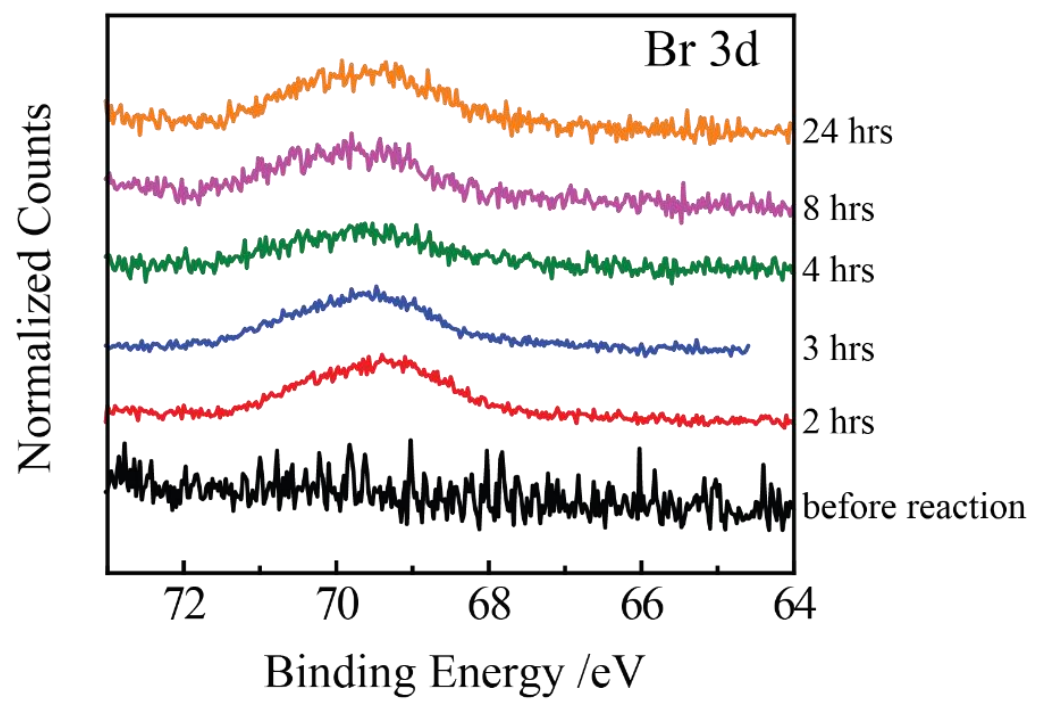

Figure 6.1 $\mathrm{Br} 3 \mathrm{~d}$ XP spectra of films casted from a GO suspension removed from a reaction mixture with 4-bromoaniline after the listed times.

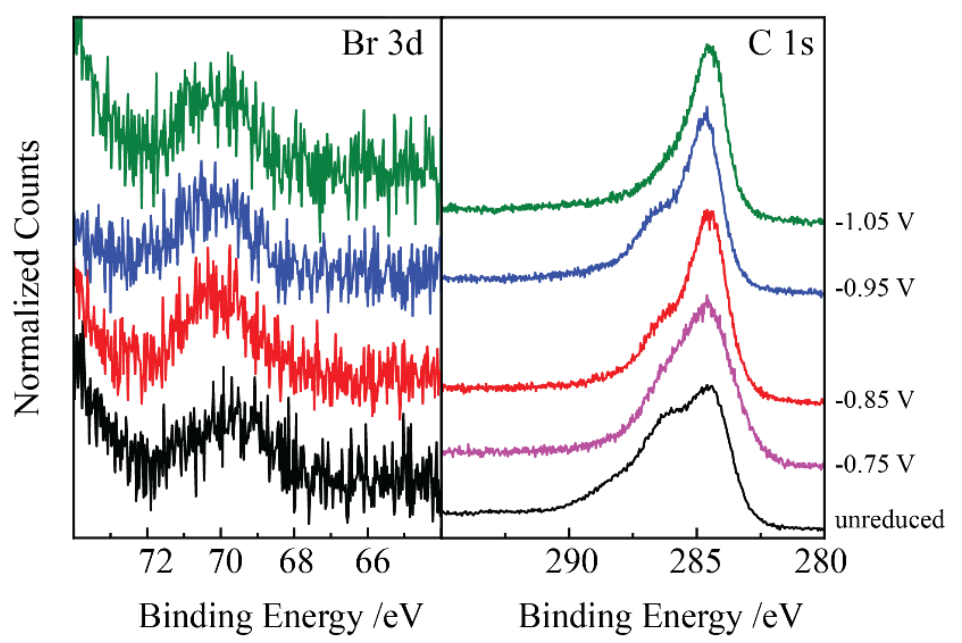

Figure 6.2 $\mathrm{Br} 3 \mathrm{~d}$ XP spectra (left) and C 1s XP spectra (right) of films casted on FTO from a suspension of $\mathrm{GO}$ after reaction with 4-bromoaniline. Each film was cycled in a $1 \mathrm{M} \mathrm{KCl}$ solution containing $1 \mathrm{mM} \mathrm{HCl}$ between open circuit potential and the potentials listed to the right in the figure three times.

Alkoxysilane grafting to GO. The second grafting method investigated in this thesis involves the reaction of an alkoxysilane with the alcohol groups of GO. It is well known that alkoxysilanes react with surface alcohol groups. These types of molecules are often used to modify 
glass and other oxide surfaces. ${ }^{328-330}$ It has also been shown previously that alkoxysilanes graft specifically to GO. ${ }^{54}$ Trimethoxyphenylsilane (TMPS) was chosen as a proof-of-concept because the phenyl group was thought to closely model the sterics and electronics of the ligands of $\mathbf{1}$. Reaction conditions using a slurry of GO and a thin film of GO were both investigated. Figure 6.3 (top left) shows the Si 2p XP spectrum of a sample of GO taken from a slurry stirred with TMPS in methanol at $70^{\circ} \mathrm{C}$ in a sealed vessel for 20 hours. Figure 6.3 (bottom left) shows the Si $2 p$ spectrum of a thin film of GO that was immersed in a solution of TMPS at $70^{\circ} \mathrm{C}$ for 20 hours in a sealed vessel. Both spectra show a distinct Si $2 p$ signal approximately at the binding energy of $\mathrm{SiO}_{\mathrm{x}}$. Reaction on a thin film of GO results in a lower $\mathrm{Si} 2 \mathrm{p}$ peak and a broader $\mathrm{C} 1 \mathrm{~s}$ peak. This suggests the quantity of TMPS is lower under these conditions because the bulky TMPS molecule likely cannot penetrate within the GO film and therefore only reacts at the exposed surface. Furthermore, the $\mathrm{C} 1 \mathrm{~s}$ signal for the GO that had been mixed as a slurry with TMPS is significantly narrowed, indicating a reduction in the overall oxide character of the material. On the other hand, the width of the $\mathrm{C} 1 \mathrm{~s}$ signal of the GO thin film that had been immersed in a solution of TMPS is more similar to that of unreacted GO. (Both C 1s spectra are overlaid with an example $\mathrm{C} 1 \mathrm{~s}$ spectrum of unreacted GO in Figure 6.3 (top and bottom right).)

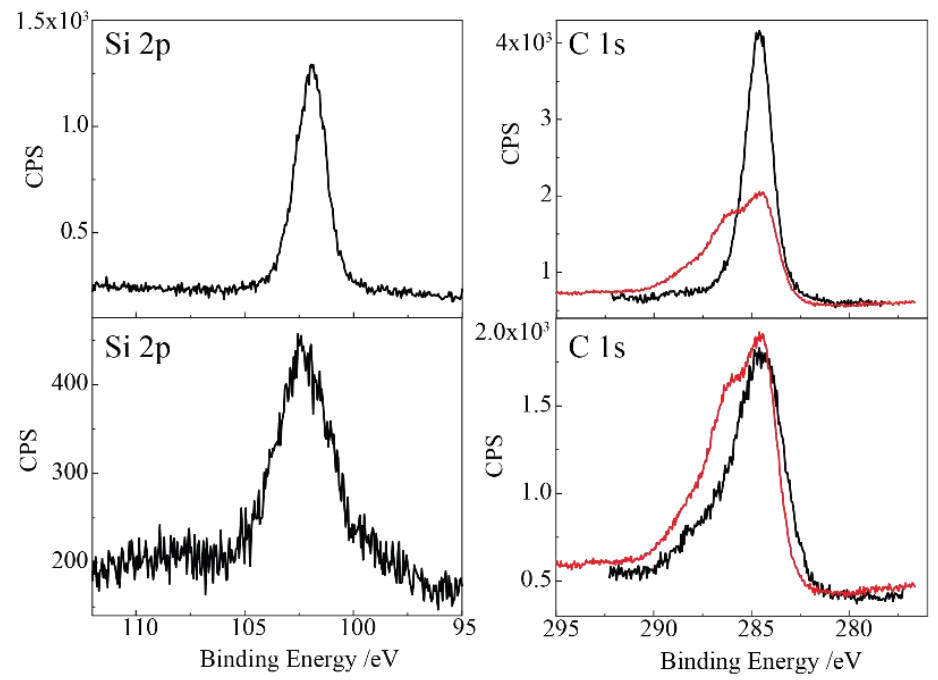

Figure 6.3 Si 2p (left) and C 1s (right) XP spectra of GO films after reaction with TMPS. The top spectra are from a slurry of GO stirred in a solution of TMPs, and the bottom spectra are from a thin film of GO immersed in a solution of TMPS. In red are representative C 1s spectra of an unreacted GO film. 
Future research should investigate the ability of the TMPS-modified GO to undergo reduction to TMPS-modified RGO. Since the linkage requires an oxygen functional group on GO, it may be labile under reductive conditions; therefore, the reduction potential must be precisely controlled in order to retain the TMPS linkage but remove any other oxygen functional group on GO.

Click reaction on $G O$. The third grafting method involves two sequential steps to modify GO. The first is attachment of an azide moiety, which was easily accomplished by following a well-cited procedure using sodium azide in water followed by freeze-drying and washing the resultant solid with water. ${ }^{331}$ FTIR spectroscopy was used to analyze the azide content. The spectra of GO before and after the azidification are shown in Figure 6.4. After reaction there is a distinct band at about $2121 \mathrm{~cm}^{-1}$, characteristic of the antisymmetric $\mathrm{N}=\mathrm{N}=\mathrm{N}$ stretch. For comparison, the stretching frequency of pure sodium azide is observed at about $2104 \mathrm{~cm}^{-1}$. XPS survey spectra of this material revealed a significant sodium signal. This sodium was removed by washing the material with hydrochloric acid (as described in the Methods section of this chapter). The azide IR stretch remained after washing with $\mathrm{HCl}$.

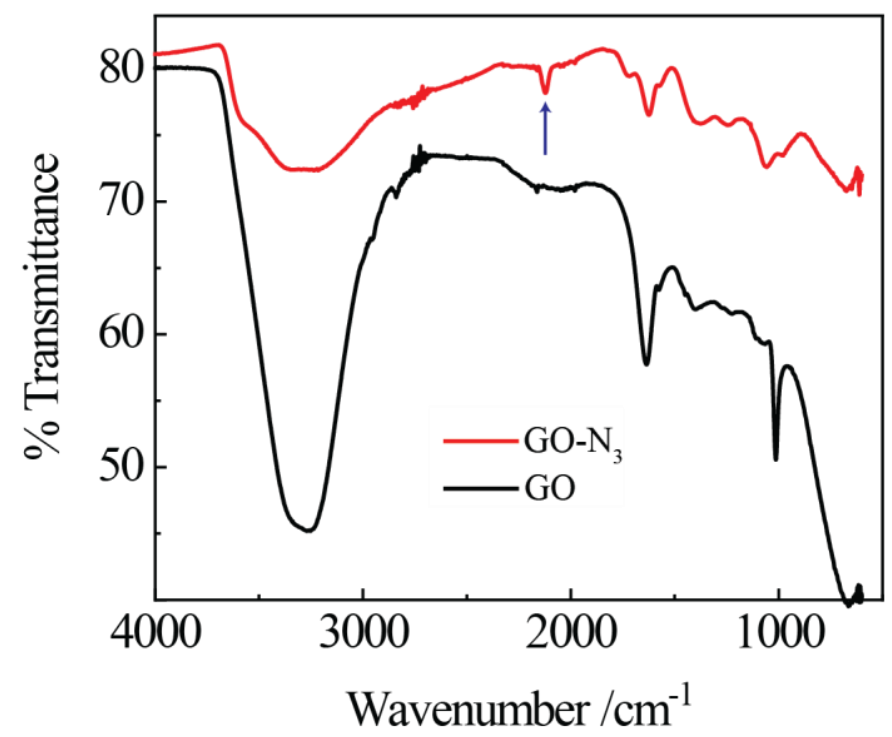

Figure 6.4 FTIR spectra of GO (black) and GO after reaction with sodium azide (red). The arrow points to the N-N-N stretching peak. 
The second step in this method involves grafting an alkyne-containing molecule via a copper-catalyzed click reaction. This step was less straightforward. 6-Iodo-1-hexyne was used for a proof-of-concept, and several attempts using various reaction conditions were made. Every attempt at using a dissolved copper species as the catalyst (e.g. $\mathrm{CuBr}, \mathrm{CuSO}_{4}$ ) resulted in a copper deposit on the surface of the film, detectable by XPS and sometimes even by eye. The copper wire catalyzed click reaction following a modification of the procedure in Reference 332 resulted in the cleanest and apparently most successful click reaction. Shown in Figure 6.5 are the XP spectra of the I $3 \mathrm{~d}$ and $\mathrm{Cu} 2 \mathrm{p}$ regions. After 3 days of reaction there was a small but discernable iodine signal and a minimal copper signal, showing that the reaction was slow but successful. Future work should focus on improving the kinetics of this reaction. Addition of an appropriate ligand may facilitate $\mathrm{Cu}^{0}$ dissolution, or reaction with a slurry of GO-N 3 may improve reaction kinetics by allowing direct contact of the azide with the copper surface.

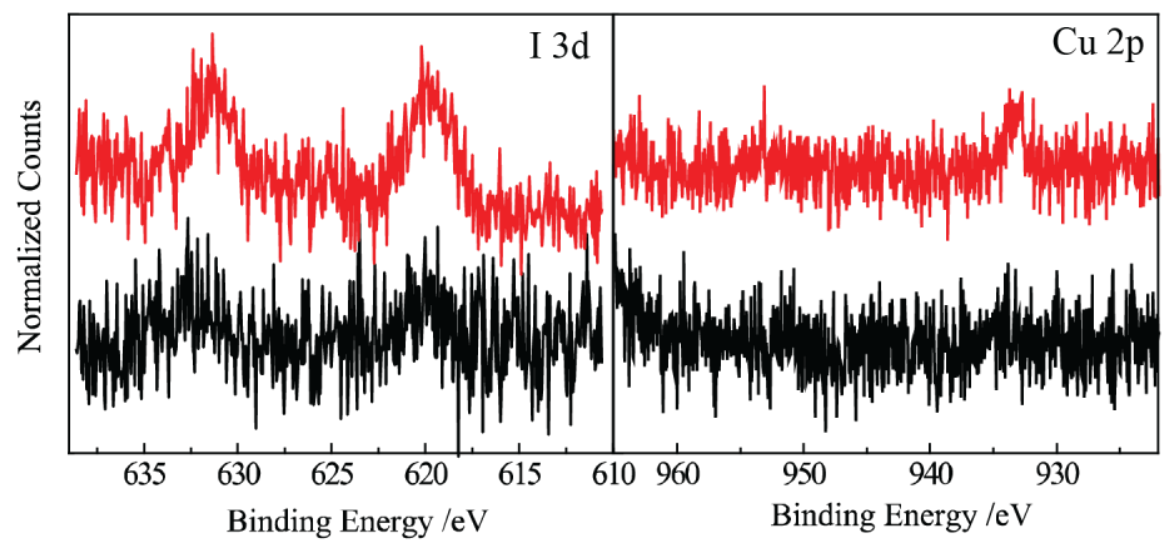

Figure 6.5 I 3d (left) and $\mathrm{Cu} 2 \mathrm{p}$ (right) spectra of a GO-N 3 film after stirring in a solution of 6iodo-1-hexyne with a copper wire wrapped around the stir bar (red) and after stirring in a solution of 6-iodo-1-hexyne with a normal stir bar.

Current and future work. Current efforts are focused on synthesizing a derivative of the benzenedithiolate ligand of $\mathbf{1}$ that contains one of the functional groups required to perform the grafting reactions above, i.e. an amine, an alkyne, or a trimethoxysilane. Future work should focus on improving the grafting reactions listed above. Specifically, the silane grafting reaction needs to be optimized to give a high surface coverage without saturating all surface oxide groups (which would likely influence the conductivity of the RGO). Furthermore, good reduction conditions for silane-grafted GO need to be identified. Likely electrochemical reduction will be the most effective 
route as it is the most controlled process. Additionally, the click reaction needs optimization. Surface coverage is low and reaction kinetics are slow. Kinetics can likely be improved by reaction in a slurry of GO-N 3 or by using an appropriate ligand to solvate $\mathrm{Cu}^{0}$.

Once a modified catalyst is synthesized and the appropriate grafting method optimized, the catalyst can then be grafted to RGO thin films on electrode surfaces and electrocatalysis can be compared to electrocatalysis of the physisorbed systems with the expectation that retention of the catalyst on the surface will be dramatically improved by grafting.

\subsection{In situ $x$-ray absorption measurements on adsorbed cobalt bis(dichlorobenzenedithiolate)}

X-ray absorption near edge structure (XANES) and extended X-ray absorption fine structure (EXAFS) measurements at the cobalt K-edge were conducted of the catalyst $\mathbf{1}$ adsorbed to carbon paper electrodes using beamline 11-2 at SSRL at the Stanford Linear Accelerator Center. Carbon paper electrodes were cut from a larger sheet and immersed in a vial containing a $5 \mathrm{mM}$ solution of 1 in acetonitrile. These electrodes were soaked for at least 2 hours but up to 2 days before measurements were conducted. Measurements were performed by immersing the electrode in a cell containing a $0.1 \mathrm{M} \mathrm{KPF}_{6}$ electrolyte solution with a graphite rod counter electrode and a $\mathrm{Ag} \mid \mathrm{AgCl}$ (saturated $\mathrm{KCl}$ ) reference electrode. A photograph of the cell is shown in Figure 6.6. A Kapton film is glued (with super glue) across the window in the center. The carbon paper electrode is positioned in front of that window. The solvent thickness at that point is $1 \mathrm{~mm}$; however, this was found to be too thick to achieve a signal, so a stack of small squares of tape (electroplater's tape) was placed on the back window of the cell to push the electrode forward against the front window to reduce the solvent layer in front of it. This method of sandwiching the electrode between the tape layer and the front window must inhibit diffusion of species in solution to the electrode surface; however, we deemed this to be insignificant considering the porosity of the electrode itself.

XANES measurements were taken at four different $\mathrm{pH}$ values (acidified with trace metal

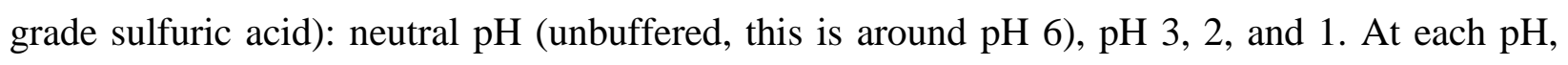
measurements were taken at a range of potentials between open circuit potential (usually around $+0.3 \mathrm{~V}$ ) and $-0.9 \mathrm{~V}$, vs $\mathrm{Ag} \mid \mathrm{AgCl}$. Under each set of conditions, three subsequent scans were taken, normalized, and averaged. One set of EXAFS measurements was also conducted on a sample in a 
$\mathrm{pH} 1$ solution at $-0.8 \mathrm{~V}$ every 30 minutes over the course of 10 hours to determine whether degradation occurs under these conditions.
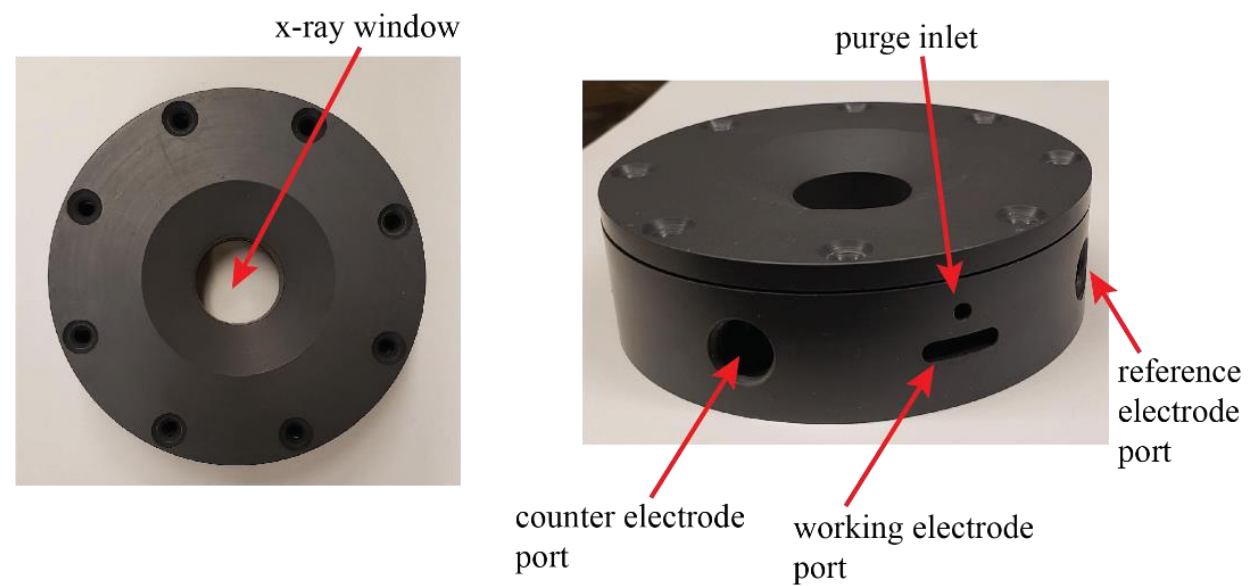

Figure 6.6 Photograph of the electrochemical cell used for XAS experiments. Front view (left) and top view (right). Kapton film was glued (using super glue) across the window in the view on the left. The electrodes were inserted through the holes on the top of the cell in the view on the right and the solution was purged via an $\mathrm{N}_{2}$ line inserted through the smallest round hole. The cell is not air-tight, but a blanket of $\mathrm{N}_{2}$ was kept flowing over the top of the solution during experiments.

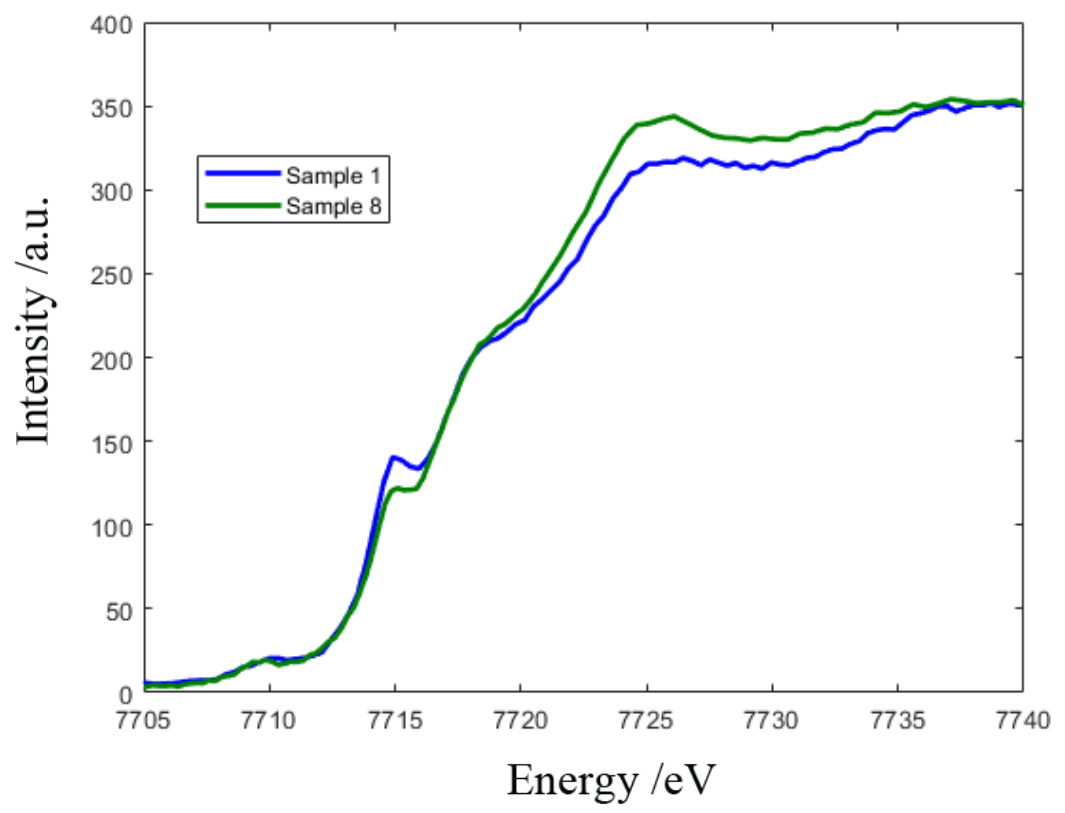

Figure 6.7 Overlay of normalized Co K-edge XANES spectra for two different samples at neutral $\mathrm{pH}$ (no added acid). Sample 1 was taken after soaking the electrode in a solution of $\mathbf{1}$ for about 4 hours on day 1, and Sample 8 was taking after soaking the electrode for about 2 days on day 5. The electrolyte was deaerated $0.1 \mathrm{M} \mathrm{KPF}_{6}$. 
After plotting the XANES data it became apparent that some small sample to sample variations had occurred, as well as variations within a single sample after holding at negative potentials. For example, Figure 6.7 shows the data for two different samples, both at neutral pH and at open circuit potential. The spectrum for Sample 1 was taken first, and the spectrum for Sample 8 was taken about 4 days later. The broader feature at $\sim 7715 \mathrm{eV}$ and the increased amplitude at $7725 \mathrm{eV}$ are consistent with coordination environment changes, from largely 4coordinate initially to more 5-coordinate after 4 days. There are two possible explanations for this finding. First, since the same solution of $\mathbf{1}$ was used to soak electrodes during the week at the beam line, it is possible that the complex underwent a change in solution over time. Some kind of aggregation or polymerization may have occurred. However, since the color of the solution appeared to remain unchanged, I believe this to be unlikely. On the other hand, since Sample 1 and Sample 8 were soaked in the solution of $\mathbf{1}$ for different amounts of time (about 4 hours and about 2 days, respectively) it is possible that longer soaking times allow for a change to occur on the surface of the carbon electrode. For example, 1 may coordinate a native oxide species on the carbon electrode, becoming 5-coordinate. Interestingly, this change in $\mathbf{1}$ over time, either on the surface or in solution, does not appear to affect the electrochemistry. Electrochemical measurements obtained from these carbon electrodes were difficult to interpret due to their high surface areas and likely large potential gradients across the electrodes. However, in previous studies on smaller, planar carbon electrodes, no change in electrochemistry related to a variation in soaking times was observed.

In comparing spectra for one sample at different potentials, it is also apparent that small, irreversible changes occur when moving to low potentials. Figure 6.8 shows spectra at a series of potentials at each $\mathrm{pH}$ point. These series of data were taken starting at open circuit potential (OCP) and then moving progressively more negative, then returning to more positive potentials, ending at OCP again. For example, for the sample at neutral $\mathrm{pH}$, a spectrum was taken at OCP; then the potential was held at $-0.4 \mathrm{~V}$ and a spectrum was taken; then it was held at $-0.6 \mathrm{~V}$ and a spectrum was taken; then $-0.8 \mathrm{~V},-0.9 \mathrm{~V}$, back to $-0.4 \mathrm{~V}$, and finally OCP again. Each spectrum was an average of three scans each of which took about 10 minutes to complete, totaling 30 minutes for a single spectrum. It is apparent from these data that some small changes have occurred at OCP after ramping to negative potentials. In most cases, upon return to $\mathrm{OCP}$, the structure looks more 5- or 
6- coordinate than initially (by the less prominent feature at about $7715 \mathrm{eV}$ ). This could be explained by the cobalt complex coordinating at the axial position to an oxide group on the carbon

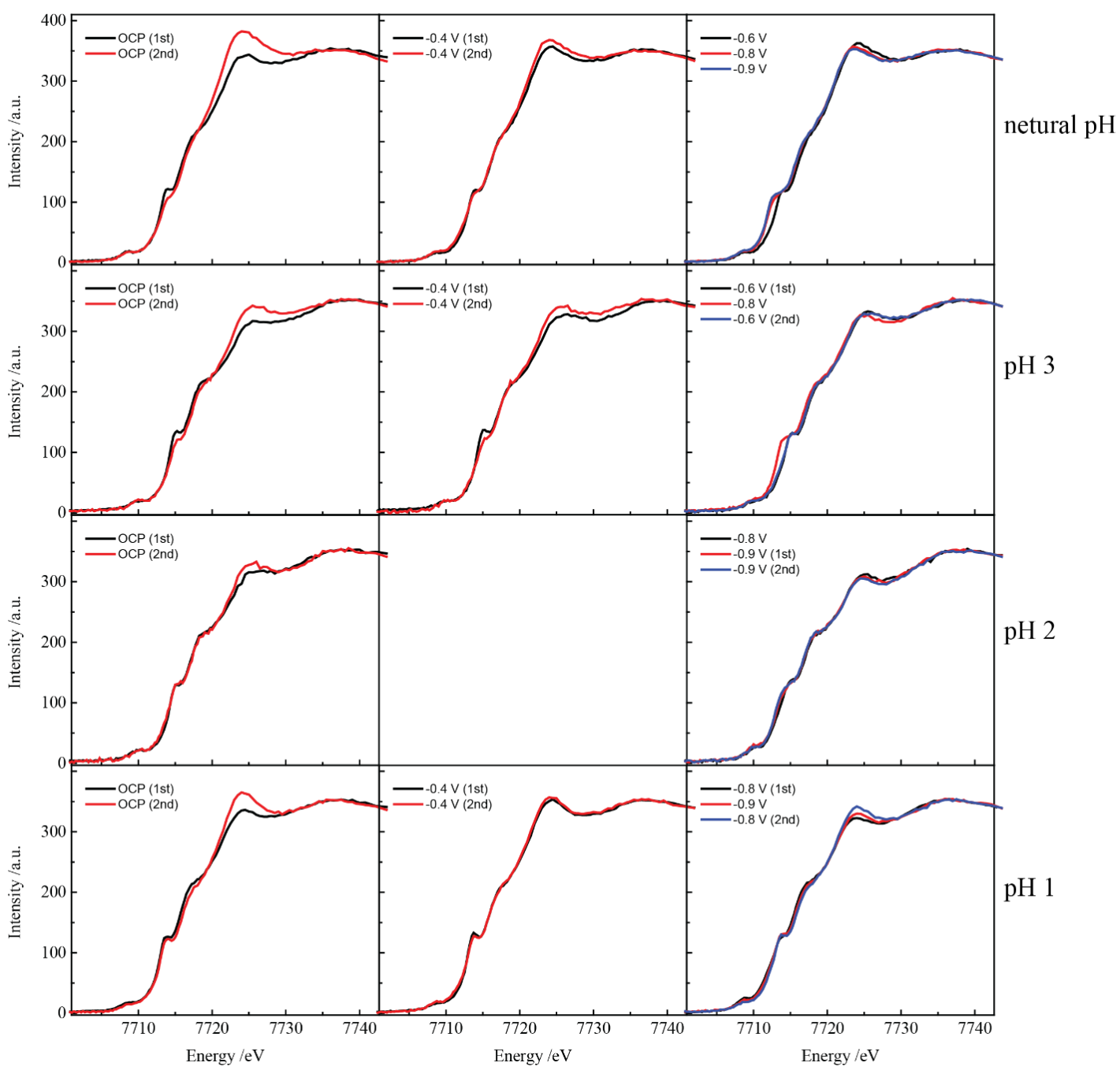

Figure 6.8 Overlay of Co K-edge XANES spectra with the electrode held at various potentials at each $\mathrm{pH}$. Each electrode was first held at OCP, then brought to $-0.4 \mathrm{~V}$, then $-0.8 \mathrm{~V}$, then $-0.9 \mathrm{~V}$, and then it was brought back through the potentials in reverse order, ending at OCP. Hence, OCP (1st) was the first spectrum and OCP (2nd) the last spectrum taken in each case. (Note that at $\mathrm{pH}$ 2, no data was taken at $-0.4 \mathrm{~V}$, hence the missing spectra.) The electrolyte was deaerated $0.1 \mathrm{M}$ $\mathrm{KPF}_{6}$ acidified with sulfuric acid. Each spectrum was taken on the same spot on the same (i.e. the sample position was not moved between scans).

paper electrode. It is also possible that further intercalation occurs when the electrode is held at negative potentials and that this intercalation sufficiently alters the structure to produce the spectral variations observed in Figure 6.8. Further EXAFS measurements and fittings can help determine 
the precise structure of $\mathbf{1}$ before and after ramping to negative potentials. However, at this time it is not clear whether these small changes are purely electrochemical based (i.e. whether ramping to negative potentials is enough to change the spectrum of $\mathbf{1}$ irreversibly) or whether it is a combination of negative potentials and illumination by the x-ray beam that causes irreversible damage to the sample. If it is the former case, this is of scientific interest and merits further study. If it is the latter case, experiments may need to be performed on a less intense beamline to limit beam damage to the sample. Further experiments are needed to determine the exact origin of this observation.

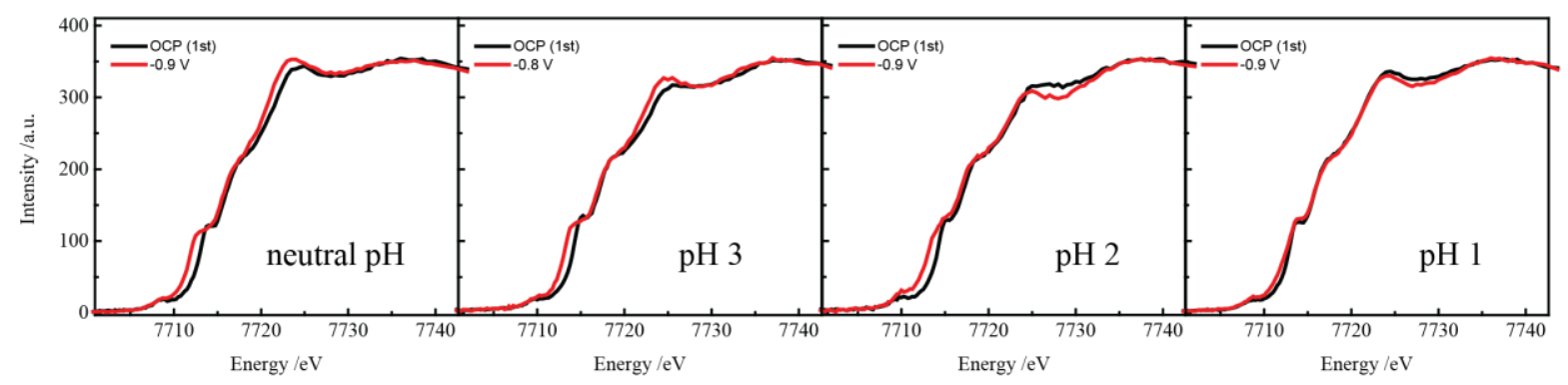

Figure 6.9 Overlays of Co K-edge XANES spectra for individual carbon paper electrodes with adsorbed 1 at the highest (OCP) and lowest (-0.9 or $-0.8 \mathrm{~V}$ ) potentials over the range of $\mathrm{pH}$ values. The electrolyte was deaerated $0.1 \mathrm{M} \mathrm{KPF}_{6}$ acidified with sulfuric acid.

In Figure 6.9, the spectra for each $\mathrm{pH}$ point at OCP and at the lowest potential are overlaid. One important takeaway from these results is that the reduction of $\mathbf{1}$ can be seen at all pH points. That is, a Co(II) species can be identified even under low $\mathrm{pH}$ when catalysis is fast (although smaller amounts of $\mathrm{Co}$ (II) are present in this case). The reduction is very apparent by the shift of the edge to lower energy in neutral $\mathrm{pH}, \mathrm{pH} 3$, and $\mathrm{pH}$ 2. In the $\mathrm{pH} 1$ solution, the edge shift is less dramatic but still occurs to a small extent. This is likely because catalysis in $\mathrm{pH} 1$ solutions is fast, meaning the reduced cobalt species is transient. Because the XANES measurement is an average of all cobalt species over the timescale of the scans, the spectrum then shows more oxidized cobalt character than reduced cobalt character. This implies that electron transfer to the Co(III) catalyst is the rate-limiting step for proton reduction catalysis under these conditions. Based on this finding, further XAS experiments are merited. If inconsistencies in the data and data collection can be limited, we may be able to derive the structure of the reduced $\mathrm{Co}$ (II) species at low $\mathrm{pH}$ and determine whether this is a protonated intermediate in the catalytic cycle. 
Lastly, Figure 6.10 shows a fit of a set of EXAFS data of a carbon electrode with adsorbed $\mathbf{1}$ in a pH 1 solution held at $-0.8 \mathrm{~V}$. This electrode was soaked for about 2 days in the solution of $\mathbf{1}$ before conducting the EXAFS measurements. The XANES spectra of this electrode, taken before the EXAFS spectrum, are shown in Figures 6.8 and 6.9 (at pH 1). Approximately 20 scans were taken and averaged to produce a single spectrum, which was fitted with a shell of four sulfur atoms at $2.19 \AA$. This bond distance matches the crystal structure for $1 .{ }^{248}$ The peak at about $3.8 \AA$ is due to the near linearity of the Co---C---C interaction $\left(164^{\circ}\right)$, which gives rise to a multiple-scattering process. This fit seems to suggest that the cobalt species is the original 4-coordinate square planar bis(benzenedithiolate) complex or something extremely similar. This electrode was soaked for a long time and held at a negative potential for a long time, which has been shown by XANES data to induce a change in the structure. One possible explanation for this phenomenon is that the cobalt species that initially adsorbs to the carbon electrode is coordinated axially to a native oxide species on the electrode and that this bond is broken at low potential. In this case, the change that is seen in the XANES spectra after ramping to negative potentials at low $\mathrm{pH}$ could be due in part to a return to the four-coordinate, $\mathrm{Co}$ (III) state. The changes seen at neutral $\mathrm{pH}$, involving a shift in edge energy in addition to a change in shape, are more consistent with reduction of Co. This, of course, merits further study. EXAFS spectra at each potential and $\mathrm{pH}$ point should be collected and fitted to obtain structural information.
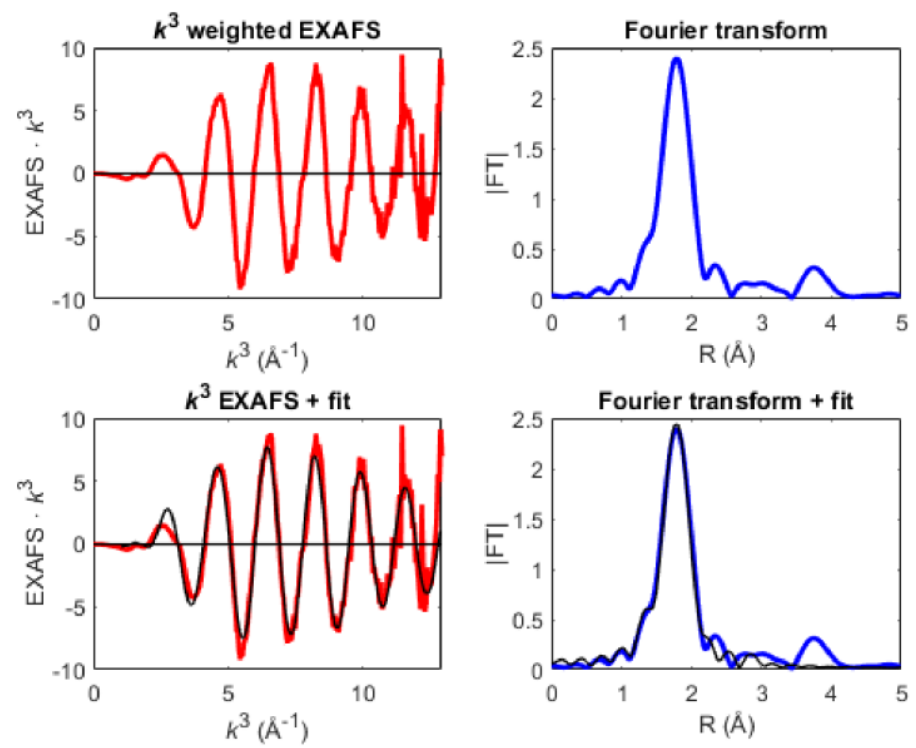

Figure 6.10 Fitted EXAFS data of a carbon paper electrode with 1 adsorbed, held at $-0.8 \mathrm{~V}$ vs $\mathrm{Ag} \mid \mathrm{AgCl}$. The electrolyte was deaerated in $0.1 \mathrm{M} \mathrm{KPF}_{6}$ and acidified to $\mathrm{pH} 1$ with sulfuric acid. Approximately 20 scans were taken and averaged. 
Future work. Further XANES and EXAFS data should be collected on this same system, but the soaking times for each electrode should be kept precisely the same. Additionally, the solution of $\mathbf{1}$ used for soaking should be either checked by UV-visible absorption spectroscopy daily to ensure it is not degrading, or the solution should be remade daily to prevent degradation. Experiments should be also conducted to determine whether an irreversible electrochemical change occurs on the adsorbed catalyst when ramping to negative potentials or if the x-ray beam itself damages the sample at negative potentials. This can be accomplished by (1) taking a spectrum at OCP, (2) ramping to a negative potential and taking a spectrum, (3) returning to OCP and taking a spectrum, and then (4) moving to a new spot on the sample and taking a spectrum. If the spectrum at the new spot is the same as spectrum 3, then the irreversible change is purely electrochemical in nature. If it is instead the same as spectrum 1, then the change is partially beam-induced.

EXAFS spectra should then be collected at different $\mathrm{pH}$ values and different applied potentials to more precisely determine the structure of the catalyst before and after the irreversible change occurs. Additionally, XPS experiments may also help understand the changes we see in the cobalt species. For example, the additional peak at $787 \mathrm{eV}$ seen in the XP spectrum of 1 on RGO in Chapter 3 (Figure 3.2) may be related to the change in the XANES data after long soaking times. Specifically, if longer soaking times result in a change in the way $\mathbf{1}$ binds to the surface, this may result in two different cobalt XPS binding energies.

\subsection{Heterogeneous photoelectrocatalysis using p-GaP photoelectrodes with an electrocatalyst adsorbed to an RGO thin film}

Photoelectrochemical water splitting is a promising clean source of hydrogen gas as the reaction can be driven partially or entirely by sunlight. The RGO films developed in Chapter 2 and modified with a proton reduction catalyst in Chapters 3 and 4 can be used on a semiconductor photoelectrode for photoelectrocatalytic proton reduction. Because RGO films are highly transparent, they allow an underlying semiconductor to absorb sunlight, and because of their electronic conductivity and ability to bind a molecular catalyst, they can facilitate electron transfer from the excited semiconductor electrode to the catalyst. Some preliminary work using p-type gallium phosphide as an electrode material is presented here. 


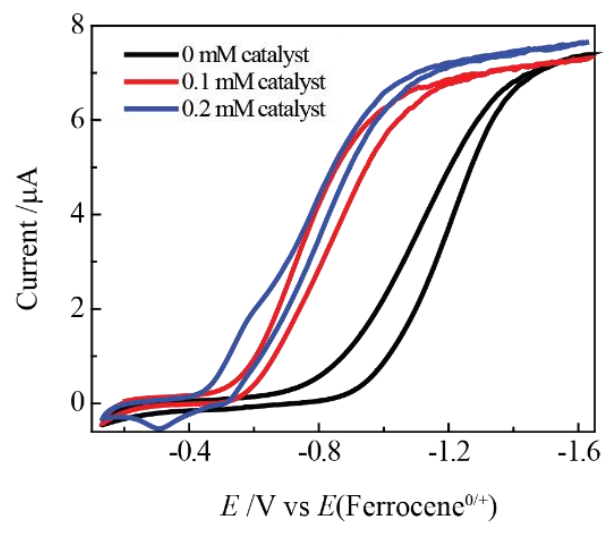

Figure 6.11 Photocurrent response of a p-GaP(100) photoelectrode in deaerated $0.1 \mathrm{M}$ tetrabutylammonium perchlorate in acetonitrile with $10 \mathrm{mM}$ trifluoroacetic acid with various concentrations of 1. A platinum mesh counter electrode and platinum wire quasi-reference electrode were used. The scan rate was $50 \mathrm{mV} / \mathrm{s}$ and the light intensity was $100 \mathrm{~mW} / \mathrm{cm}^{2}$ white light.

Initially, the catalyst 1 (discussed in Chapters 3 and 4) was dissolved in electrolyte solution and bare planar p-GaP(100) wafers were used as electrodes. The electrolyte consisted of $0.1 \mathrm{M}$ tetrabutylammonium perchlorate (TBAP) in acetonitrile with $10 \mathrm{mM}$ trifluoroacetic acid (TFA). The photocurrent responses of this system under $100 \mathrm{~mW} / \mathrm{cm}^{2}$ white light (halogen lamp) are shown in Figure 6.11 Upon addition of $0.1 \mathrm{mM} 1$ to the solution, the onset current shifted positive by about $400 \mathrm{mV}$. After raising the concentration of 1 to $0.2 \mathrm{mM}$, a pre-wave appeared as a shoulder at about $-0.5 \mathrm{~V}$. It is not clear what process caused this wave.

After verifying the ability of $\mathrm{p}-\mathrm{GaP}$ to facilitate charge transport to $\mathbf{1}$ under illumination thereby catalyzing proton reduction, GO films were dropcasted on methylated p-GaP(111A) electrodes (methylated following the Grignard procedure in Reference 333) and reduced using cobaltocene (as in Chapter 2). It should be noted that depositing RGO films on bare p-GaP electrodes caused severe degradation of the GaP surface, resulting in no photocurrent response; therefore, methylation of the GaP surface was performed to inhibit this degradation. The methylated GaP electrode modified with an RGO thin film was immersed in the same electrolyte solution as used in the paragraph above (0.1 M TBAP and $10 \mathrm{mM}$ TFA in acetonitrile). The photocurrent responses (Figure 6.12 were not as pristine as with a bare GaP surface; however, it is apparent that current onset occurred at a slightly more positive potential with the addition of $\mathbf{1}$. This demonstrates that charge transport from p-GaP to $\mathbf{1}$ can occur through a thin RGO film. 


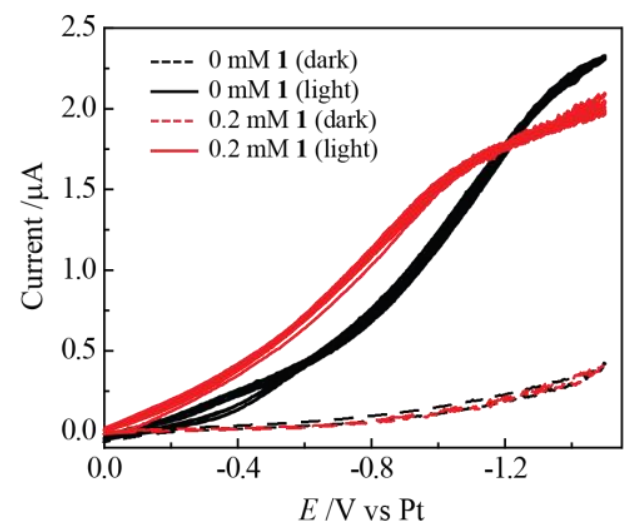

Figure 6.12 Photocurrent response of a methylated p-GaP(111A) photoelectrode with an RGO film in deaerated $0.1 \mathrm{M}$ tetrabutylammonium perchlorate in acetonitrile with $10 \mathrm{mM}$ trifluoroacetic acid with various concentrations of $\mathbf{1}$. A platinum mesh counter electrode and platinum wire quasireference electrode were used. The scan rate was $50 \mathrm{mV} / \mathrm{s}$ and the light intensity was $100 \mathrm{~mW} / \mathrm{cm}^{2}$ white light. The dashed lines are the current in the dark.

The next experiments attempted physisorption of $\mathbf{1}$ to RGO surfaces on p-GaP. The photocurrent response of RGO thin films on methylated p-GaP wafers were first obtained in 0.1 $\mathrm{M} \mathrm{KPF}_{6}$ solution with $10 \mathrm{mM} \mathrm{TFA}$ in water under $100 \mathrm{~mW} / \mathrm{cm}^{2}$. These wafers were then immersed in a $5 \mathrm{mM}$ solution of $\mathbf{1}$ in acetonitrile in an inert-atmosphere glovebox for 40 minutes. After rinsing and drying under a nitrogen stream the photocurrent response was again obtained. Surprisingly, the onset of current was shifted to more negative potentials than before soaking in catalyst (Figure 6.13. This suggests that soaking in the catalyst solution somehow degraded the GaP-RGO interface. Possibly trace oxygen or water in the solution damaged the GaP surface.

In a final experiment, solutions of GO and $\mathbf{1}$ were mixed before casting (following the procedure for forming embedded films in Chapter 4). This mixture was dropcasted on phenylated p-GaP wafers. The GO was reduced to RGO by holding the wafers at $-0.4 \mathrm{~V}$ vs $\mathrm{Ag} \mid \mathrm{AgCl}$ for 300 $\mathrm{s}$ in $0.1 \mathrm{M} \mathrm{KPF}_{6}$ with $10 \mathrm{mM}$ TFA in water under $100 \mathrm{~mW} / \mathrm{cm}^{2}$ white light. The onset of photocurrent with these electrodes was not different between electrodes with $\mathbf{1}$ and electrodes without 1. As can be seen in Figure 6.14, the variation between two identical electrodes exceeds the difference between electrodes with $\mathbf{1}$ and electrodes without $\mathbf{1}$.

In order to obtain more consistent results, GO films fabricated by spincasting were developed in the hopes that these would be more uniform and controllable than films fabricated 


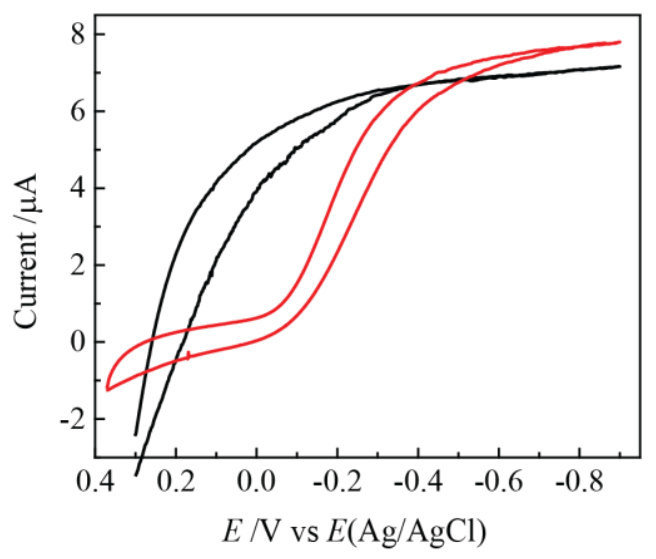

Figure 6.13 Photocurrent response of a phenylated p-GaP(111A) photoelectrode with an RGO film before (black) and after (red) soaking in a $5 \mathrm{mM}$ solution of $\mathbf{1}$ in acetonitrile in a glovebox for 30 minutes. The supporting electrolyte was $0.1 \mathrm{M} \mathrm{KPF}_{6}$ in water with $10 \mathrm{mM}$ trifluoroacetic acid. A platinum mesh counter electrode and a $\mathrm{Ag} \mid \mathrm{AgCl}$ reference electrode were used. The scan rate was $50 \mathrm{mV} / \mathrm{s}$ and the light intensity was $100 \mathrm{~mW} / \mathrm{cm}^{2}$ white light.

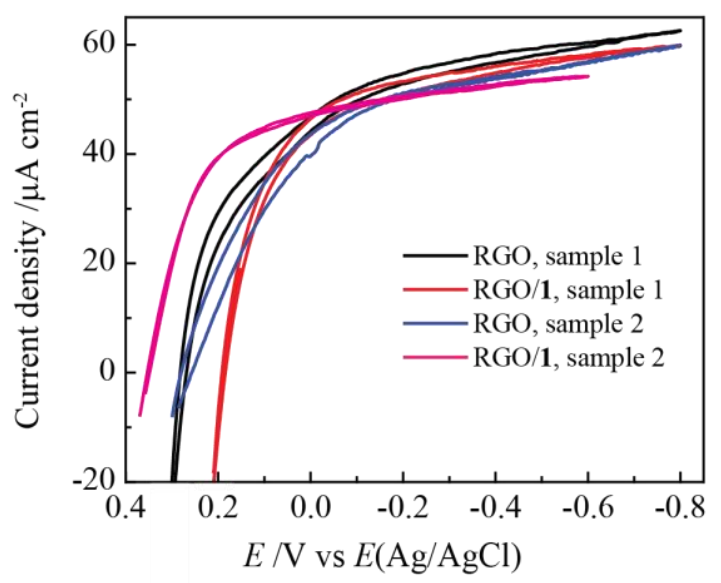

Figure 6.14 Photocurrent response of phenylated p-GaP(111A) photoelectrodes with an RGO film (black and blue) and with an RGO film embedded with $\mathbf{1}$ (red and pink). The supporting electrolyte was $0.1 \mathrm{M} \mathrm{KPF}_{6}$ in water with $10 \mathrm{mM}$ trifluoroacetic acid. A platinum mesh counter electrode and a $\mathrm{Ag} \mid \mathrm{AgCl}$ reference electrode were used. The scan rate was $50 \mathrm{mV} / \mathrm{s}$ and the light intensity was $100 \mathrm{~mW} / \mathrm{cm}^{2}$ white light.

by dropcasting. Unfortunately, however, other issues arose when using the spincasting method. GO can be spincasted on hydrophilic surfaces when suspended in methanol. Gallium phosphide surfaces can be made hydrophilic by a Grignard type functionalization resulting in a terminal 
benzyl alcohol group. However, the hydrophilic surface that was beneficial for spincasting GO was detrimental after the GO was reduced to RGO. Where GO is hydrophilic, RGO is hydrophobic and it was seen to delaminate quickly from benzyl alcohol terminated $\mathrm{GaP}$ surfaces under photoelectrochemical experiments in acidic water.

Future work. One option for spincasting adherent RGO films is to functionalize the GaP surface with a group that can alternate between hydrophobic and hydrophilic character. For example, the functional group in Figure 6.15 can rotate about the methylene bond to direct either the vinyl group (which is hydrophobic) or the alcohol group (hydrophilic) upwards. On this type of surface, when exposed to a protic solvent, like methanol, the surface group should orient with the alcohol group upward, presenting a hydrophilic surface. When the GO film is reduced to RGO, the surface bound moiety should rotate so that the vinyl group faces upward, presenting a hydrophobic surface. Developing a GaP surface functionality with these characteristics is one option to move forward with this project. Another option is to use a different deposition method to obtain highly reproducible, uniform thin RGO films. Several methods exist in the literature, such as Langmuir-Blodgett, spray-casting, and Meyer rod coating. ${ }^{81-82,334-336}$ If highly uniform, reproducible RGO thin films can be formed on $\mathrm{p}-\mathrm{GaP}$, then photoelectrochemical measurements should show better performance with 1-modified RGO films compared to plain RGO films.

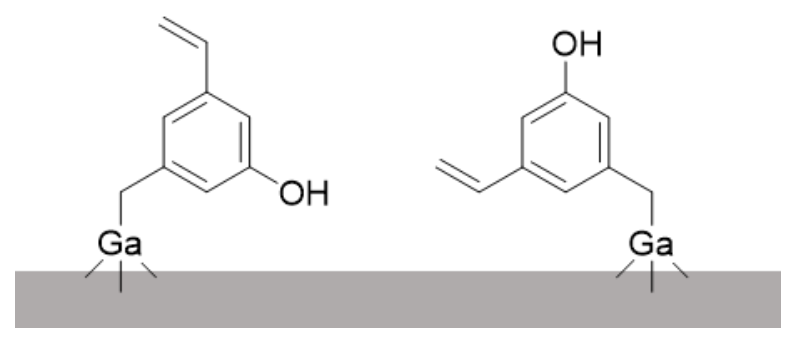

Figure 6.15 Possible functionalization of a gallium phosphide surface that can present both a hydrophobic (left) and hydrophilic (right) surface.

However, even with the poor reproducibility of photoelectrochemical measurements on RGO-modified GaP photocathodes, it is possible that any potential gain with this system in the presence of $\mathbf{1}$ is minimal. Therefore, in addition to the experiments described above, experiments using other semiconductors such as $\mathrm{p}-\mathrm{Si}$ should be conducted. Furthermore, wide-bandgap semiconductors, such as $\mathrm{TiO}_{2}$ or $\mathrm{NiO}$, can be used with an adsorbed sensitizer in addition to 1 . The 
dye crystal violet $\left(\lambda_{\max } \sim 590 \mathrm{~nm}\right)$ was shown in Chapter 2 to adsorb strongly and remain electrochemically active on RGO films. Adsorbing both crystal violet and $\mathbf{1}$ to an RGO film on a wide-bandgap semiconductor is worth investigating as a sensitized photoelectrocatalytic system.

\subsection{Passivation of n-Si photoelectrodes with RGO thin films}

Silicon is a common material for both photoanodes and photocathodes in photoelectrochemical energy conversion. When used as a photocathode, Si is highly stable, but when used as a photoanode the surface is susceptible to oxidation, especially in aqueous and oxygenated media. ${ }^{337-338}$ In order for silicon to be viable as a photoanode material for water oxidation, the surface must be passivated to prevent silicon oxidation. Chemical passivation techniques involve saturating "dangling bonds" at the surface, i.e. binding small molecules to surface defect sites. This lowers the Si surface energy and makes it less likely to react with species such as $\mathrm{O}_{2}$ or $\mathrm{H}_{2} \mathrm{O} .{ }^{339-340}$ Physical passivation involves deposition of an organic or inorganic layer on the Si surface that acts simply as a physical barrier to reactive species. ${ }^{341}$ Graphene has been shown previously to passivate $\mathrm{n}$-Si photoanode surfaces against oxidation in aqueous media. ${ }^{43,76-}$ 77 The combination of a physical passivation layer on top of a chemically passivated surface should doubly extend the lifetime of a silicon surface under oxidative conditions, by simultaneously lowering the energy of the surface atoms and physically blocking reactive species from approaching the surface. To demonstrate this effect, data are presented in this section detailing the use of RGO thin films as a physical passivation method on chemically passivated n-Si photoanodes.

Ultra-thin RGO films were prepared on n-silicon substrates by spincasting a methanolic suspension of $\mathrm{GO}$, followed by reduction to RGO in a $15 \mathrm{mM} \mathrm{CoCp} 2 / \mathrm{TFA}$ solution in the glovebox (see Chapter 2 for details on the reduction method). Spincasted films were highly uniform when the silicon surface was hydrophilic. To this end, bare silicon surfaces were either etched with $\mathrm{O}_{2-}$ plasma directly before spincasting or chemically passivated with a benzyl alcohol group through a Grignard treatment. GO suspensions were spincasted at $500 \mathrm{rpm}$ for 5 seconds, $1500 \mathrm{rpm}$ for 35 seconds, and $2000 \mathrm{rpm}$ for 1 minute. GO was reduced to RGO by immersion in a $15 \mathrm{mM}$ solution of cobaltocene with trifluoroacetic acid for 30 minutes, following the procedure in Chapter 2. 

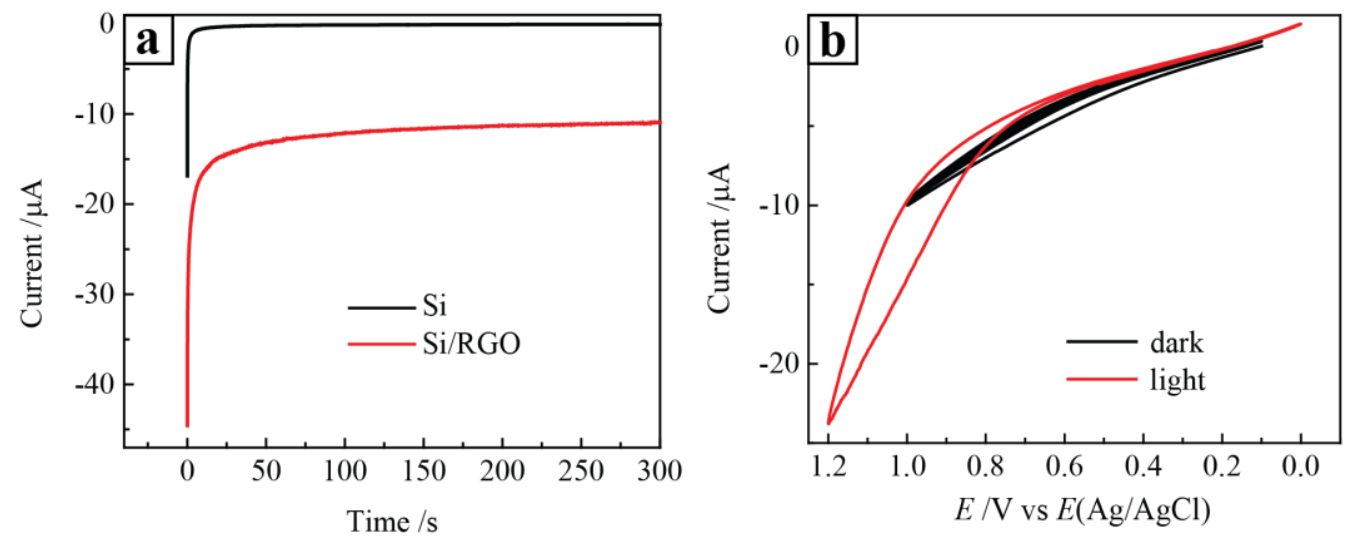

Figure 6.16 (left) Electrolysis of a silicon photoanode with (red) and without (black) an RGO thin film under $0.5 \mathrm{~V}$ and $100 \mathrm{~mW} / \mathrm{cm}^{2}$ white light. (right) Photocurrent response of the $\mathrm{Si} / \mathrm{RGO}$ photoanode after the electrolysis. The electrolyte was $0.5 \mathrm{M} \mathrm{K}_{4} \mathrm{Fe}(\mathrm{CN})_{6}$ in $0.2 \mathrm{M}$ phosphate buffer ( $\mathrm{pH} 7$ ). Platinum mesh was used as the counter electrode and $\mathrm{Ag} \mid \mathrm{AgCl}$ was used as the reference electrode.

Figure 6.16 (a) shows electrolysis data of a bare n-Si electrode (black) and a bare n-Si electrode with an RGO film (red). The electrodes were held at $0.5 \mathrm{~V}$ (vs $\mathrm{Ag} \mid \mathrm{AgCl}$ ) under 50 $\mathrm{mW} / \mathrm{cm}^{2}$ white light (halogen lamp) in a solution of $0.5 \mathrm{M} \mathrm{K} 4 \mathrm{Fe}(\mathrm{CN})_{6}$ in $0.2 \mathrm{M}$ phosphate buffer ( $\mathrm{pH} 7$ ). Platinum mesh was used as the counter electrode. Current on both electrodes dropped off extremely quickly, reaching a plateau within seconds. The initial current and the final plateau current of $\mathrm{Si} / \mathrm{RGO}$ were higher than those of bare $\mathrm{Si}$. Cyclic voltammograms of the $\mathrm{Si} / \mathrm{RGO}$ electrode after electrolysis are shown in Figure 6.16 (b). Current under illumination was not different than current in the dark at the electrolysis potential. From this data it can be concluded that the higher current from $\mathrm{Si} / \mathrm{RGO}$ compared to bare $\mathrm{Si}$ was due to an exceptionally high dark current. This may be due to the formation of a large defect layer on Si during the RGO deposition process.

Figure 6.17 shows data corresponding to silicon wafers functionalized with a benzyl alcohol group (termed $\mathrm{Si}-\mathrm{BnOH}$ ) with and without an RGO film. Passivation with the benzyl alcohol group helps to protect the silicon surface during RGO deposition. Figure 6.17a shows cyclic voltammetry of fresh $\mathrm{Si}-\mathrm{BnOH}$ and $\mathrm{Si}-\mathrm{BnOH} / \mathrm{RGO}$ electrodes in $0.5 \mathrm{M} \mathrm{K} \mathrm{Fe}_{4}(\mathrm{CN})_{6}$ in a 0.2 M phosphate buffer ( $\mathrm{pH} \mathrm{7)}$ under $50 \mathrm{~mW} / \mathrm{cm}^{2}$ white light (halogen lamp). Both show the typical square shape of light-limited current at a semiconductor. The Si-BnOH/RGO electrode photocurrent plateaued at a lower value than the $\mathrm{Si}-\mathrm{BnOH}$ electrode and the plateau region was 

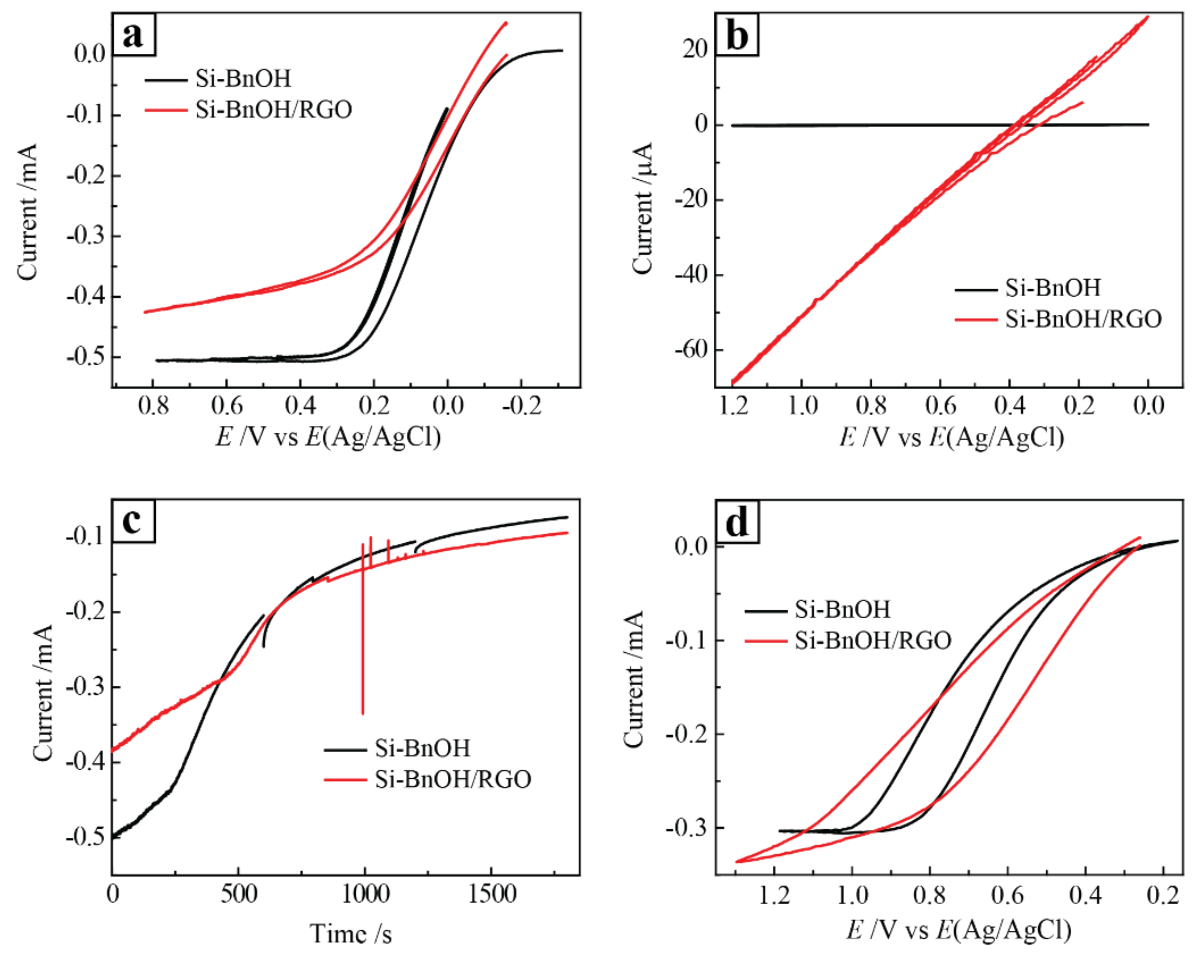

Figure 6.17 (a) Photoresponse of a silicon (111) photoanode functionalized with a benzyl alcohol group with (red) and without (black) an RGO film. (b) Dark current of the same electrodes as in (a). (c) Electrolysis under illumination at $0.5 \mathrm{~V}$ using the same electrodes as in (a) and (b). (d) Photoresponse of the electrodes after conducting the electrolysis in (c). The electrolyte was $0.5 \mathrm{M}$ $\mathrm{K}_{4} \mathrm{Fe}(\mathrm{CN})_{6}$ in $0.2 \mathrm{M}$ phosphate buffer $(\mathrm{pH} 7)$ and illumination in all cases was $50 \mathrm{~mW} / \mathrm{cm}^{2}$ white light. Platinum mesh was used as the counter electrode and $\mathrm{Ag} \mid \mathrm{AgCl}$ was used as the reference electrode.

less flat. The former can be explained by light absorbance from the RGO thin film (bulk RGO is black) and a less-than-ideal interface between RGO and $\mathrm{Si}$, which would result in a non-ideal photocurrent response. This is further supported by the dark current responses shown in Figure 6.17b. From this data the $\mathrm{Si}-\mathrm{BnOH} / \mathrm{RGO}$ electrode showed clear resistor-like behavior, suggesting that even with the chemical passivation, the $\mathrm{Si}-\mathrm{RGO}$ interface is somewhat resistive. However, the electrolysis data (Figure 6.17c) were promising. After holding each electrode at $0.5 \mathrm{~V}$ under 50 $\mathrm{mW} / \mathrm{cm}^{2}$, current at the $\mathrm{Si}-\mathrm{BnOH}$ electrode decayed sharply after about $250 \mathrm{~s}$. While the initial current at the $\mathrm{Si}-\mathrm{BnOH} / \mathrm{RGO}$ electrode was lower than $\mathrm{Si}-\mathrm{BnOH}$, it did not sharply decay until after about $500 \mathrm{~s}$. This points to the ability of RGO to stabilize silicon photoanodes under oxidative conditions. Figure 6.17d shows voltammograms under illumination after a 30-minute electrolysis at $0.5 \mathrm{~V}$ under illumination. Both voltammograms retained the ideal square shape, but the onset of 
current and plateau of current occurred at more positive potentials for both electrodes, compared to the voltammetry before electrolysis, which is indicative of surface degradation.

The final set of data shown here correspond to $\mathrm{Si}-\mathrm{BnOH}$ and $\mathrm{Si}-\mathrm{BnOH} / \mathrm{RGO}$ electrodes after having been subjected to drying on a vacuum line ( 200 mTorr) for two days as an attempt to fully dehydrate the RGO film. The ability of an RGO thin film to protect silicon wafers from oxidation can clearly be seen from this data. The electrolysis data in Figure $6.18 \mathrm{c}$ shows that the current from the $\mathrm{Si}-\mathrm{BnOH} / \mathrm{RGO}$ electrode was steady for longer than 20 minutes. At about 22 minutes, it rapidly droped off. Current from the $\mathrm{Si}-\mathrm{BnOH}$ electrode, on the other hand, rapidly droped off in under 5 minutes. Furthermore, the difference in the quality of photocurrent after a $\sim 30$ minute electrolysis can be seen in Figure $6.18 \mathrm{~b}$. While both curves shifted positive compared to before electrolysis (Figure 6.18a), the curve for $\mathrm{Si}-\mathrm{BnOH}$ shifted far more positive than Si$\mathrm{BnOH} / \mathrm{RGO}$. The electrolysis potential $(0.5 \mathrm{~V})$ was still in the plateau current region (i.e. maximum current) for $\mathrm{Si}-\mathrm{BnOH} / \mathrm{RGO}$, whereas it was at the base of the wave (minimum current) for Si-BnOH. Furthermore, Figure 6.19 shows XPS data of these surfaces. The Si oxide peak of $\mathrm{Si}-\mathrm{BnOH}$ clearly increased after electrolysis but for $\mathrm{Si}-\mathrm{BnOH} / \mathrm{RGO}$ there was little change.
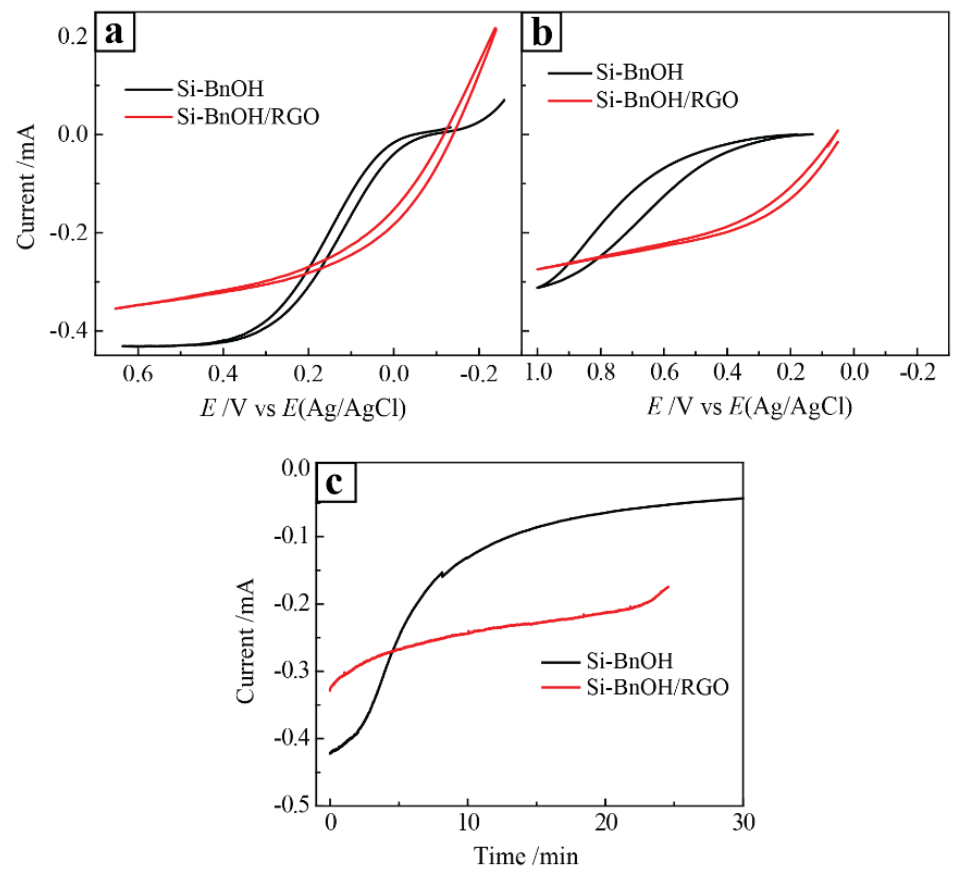

Figure 6.18 (a) Photoresponse of a silicon (111) photoanode functionalized with a benzyl alcohol group with (red) and without (black) an RGO film after drying under vacuum. (b) Photoresponse of the electrodes after conducting the electrolysis in (c). (c) Electrolysis under illumination and 0.5 $\mathrm{V}$ using the same electrodes as in (a) and (b). The electrolyte was $0.5 \mathrm{M} \mathrm{K} 4 \mathrm{Fe}(\mathrm{CN})_{6}$ in $0.2 \mathrm{M}$ 
phosphate buffer ( $\mathrm{pH} 7$ ) and illumination in all cases was $50 \mathrm{~mW} / \mathrm{cm}^{2}$ white light. Platinum mesh was used as the counter electrode and $\mathrm{Ag} \mid \mathrm{AgCl}$ was used as the reference electrode.
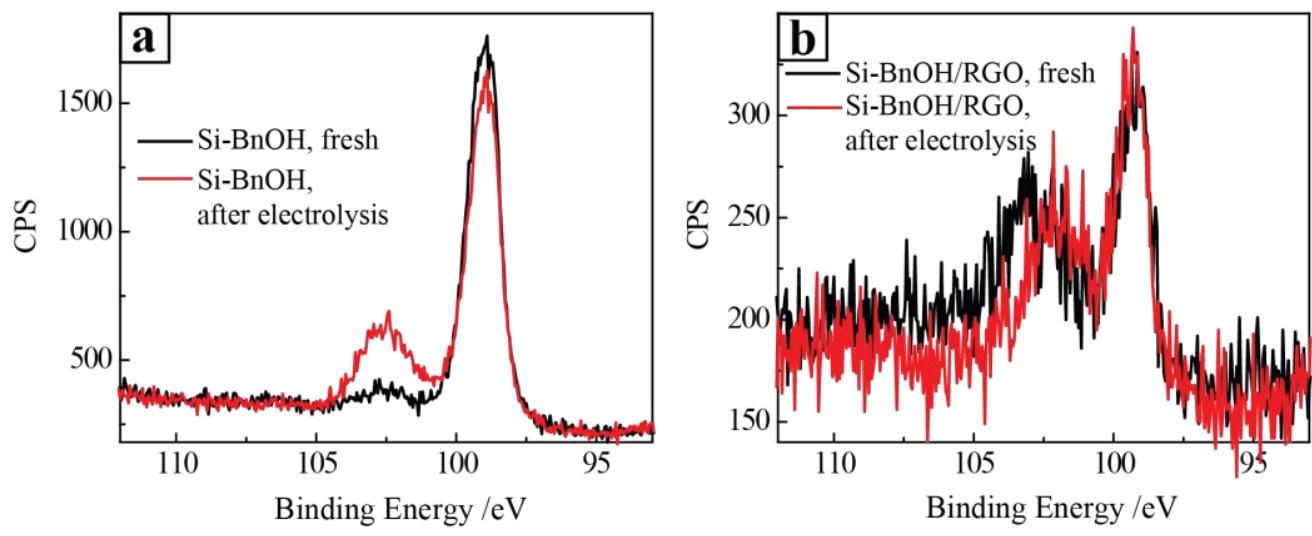

Figure 6.19 Si $2 p$ XP spectra of the silicon photoanodes before and after electrolysis under illumination. (a) Silicon (111) functionalized with a benzyl alcohol group. (b) Silicon (111) functionalized with a benzyl alcohol group and with an RGO film that had been dried under vacuum.

Future work. Unfortunately, at this point the RGO thin films became irreproducible. Spincasting resulted in spotty, thick, or nonexistent films. The cause of this was attributed to a change in the chemical functionalities of GO that had been kept in suspension over time. Further work is needed to obtain reproducible, uniform thin films of GO again. Once these films can be obtained, additional experiments showing reproducible passivation of $\mathrm{n}-\mathrm{Si}$ under oxidative conditions should be performed. The best results so far were obtained when the Si wafer was first covalently passivated (with a benzyl alcohol group) and when the RGO film was dried under vacuum before performing electrochemistry. The effect of the drying procedure should be investigated, i.e. effects of drying time and of vacuum strength. Separately, effects of film thickness can be investigated. Additional experiments should be conducted on other unstable semiconductor photoelectrodes (such as $\mathrm{GaP}$ and $\mathrm{InGaP}$ ).

Furthermore, it is worth investigating whether an RGO film can be casted on a bare semiconductor surface (i.e. with no initial chemical passivation) without damaging the surface, and whether this film alone can passivate the surface. This might be achievable, for example, by using a completely air-free and water-free suspension of GO under an inert atmosphere. Due to the hydrophilic nature of GO, it will be difficult to obtain a completely water-free suspension, 
however, with proper use of drying agents and repeated washing with dry solvents, it should be possible.

\subsection{Graphene oxide self-assembled monolayers on silicon oxide surfaces}

As discussed in Chapter 3, graphene oxide (GO) can be functionalized with small molecules in a variety of ways. Using these functionalization methods, a variety of small molecules, proteins, and nanoparticles have been tethered to GO sheets ${ }^{54,342-344}$ but little research has delved into tethering GO sheets to macroscopic surfaces. ${ }^{345}$ Tethering GO to surfaces can be beneficial in several ways, including inhibition of film delamination, allowing for formation of single-layer GO films, and pattering of single-layer GO films.

Among the many reported GO functionalization methods, alkoxysilane and amino functionalities bind to GO through the hydroxyl and epoxide groups, respectively. These grafting methods are discussed further in Chapter 3. It is also known that alkoxysilane groups bind to glass (silicon oxide) surfaces through terminal hydroxyl groups. ${ }^{330}$ Using this information, two molecular tethers were investigated for the binding of GO monolayers to silicon oxide surfaces. They are (3-aminopropyl)-triethoxysilane (APTES) and tetraethylorthosilicate (TEOS) (Figure 6.19). These species were first bound to silicon wafers containing a native oxide layer by soaking the slides in a $1 \%$ solution of the appropriate silane in toluene for 24 hours. The slides were then rinsed with clean toluene and acetone, dried, and then immersed in a homogeneous methanolic suspension of GO. In the case of APTES, the suspension was heated to about $70^{\circ} \mathrm{C}$ for 2 hours, then cooled, and the slide was rinsed with methanol and then water and dried under a nitrogen stream. In the case of TEOS, the suspension was left at room temperature for varied amounts of time.
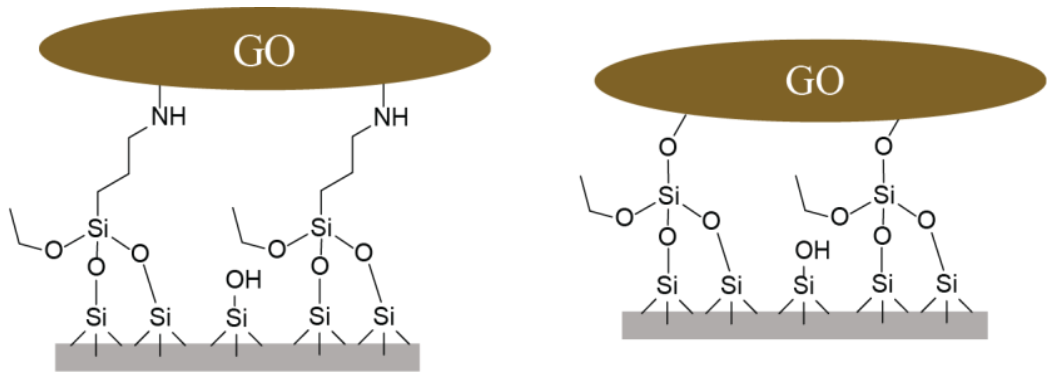

Figure 6.20 Scheme for GO monolayer attachment on silicon oxide surfaces using APTES (left) and TEOS (right). 
AFM images of the modified Si surfaces are shown in Figure 6.20. From these images it can be seen that in the case of APTES a full coverage or almost full coverage GO film was present. The film was thin enough that individual overlapping GO sheets could be distinguished, suggesting it was approximately one sheet thick. However, this should further be confirmed by examining the step height at an edge of the film. In the case of TEOS, the surface grafting reaction was much slower. After 20 hours soaking in a GO suspension, single GO sheets could be observed scattered across the surface. After 4 days in a GO suspension, a higher density of GO sheets could be observed. And after 1 week in a GO suspension even more sheets were observed. However, at this point it was apparent that the sheets had begun to aggregate in clumps leaving areas of bare glass exposed. It was hypothesized that deposition of a GO sheet on a surface leads to aggregation at that site over time, in the same way that a crystallite nucleates crystal growth in a solution. Reaction of GO with surface-bound TEOS at room temperature was too slow to out-compete aggregation.
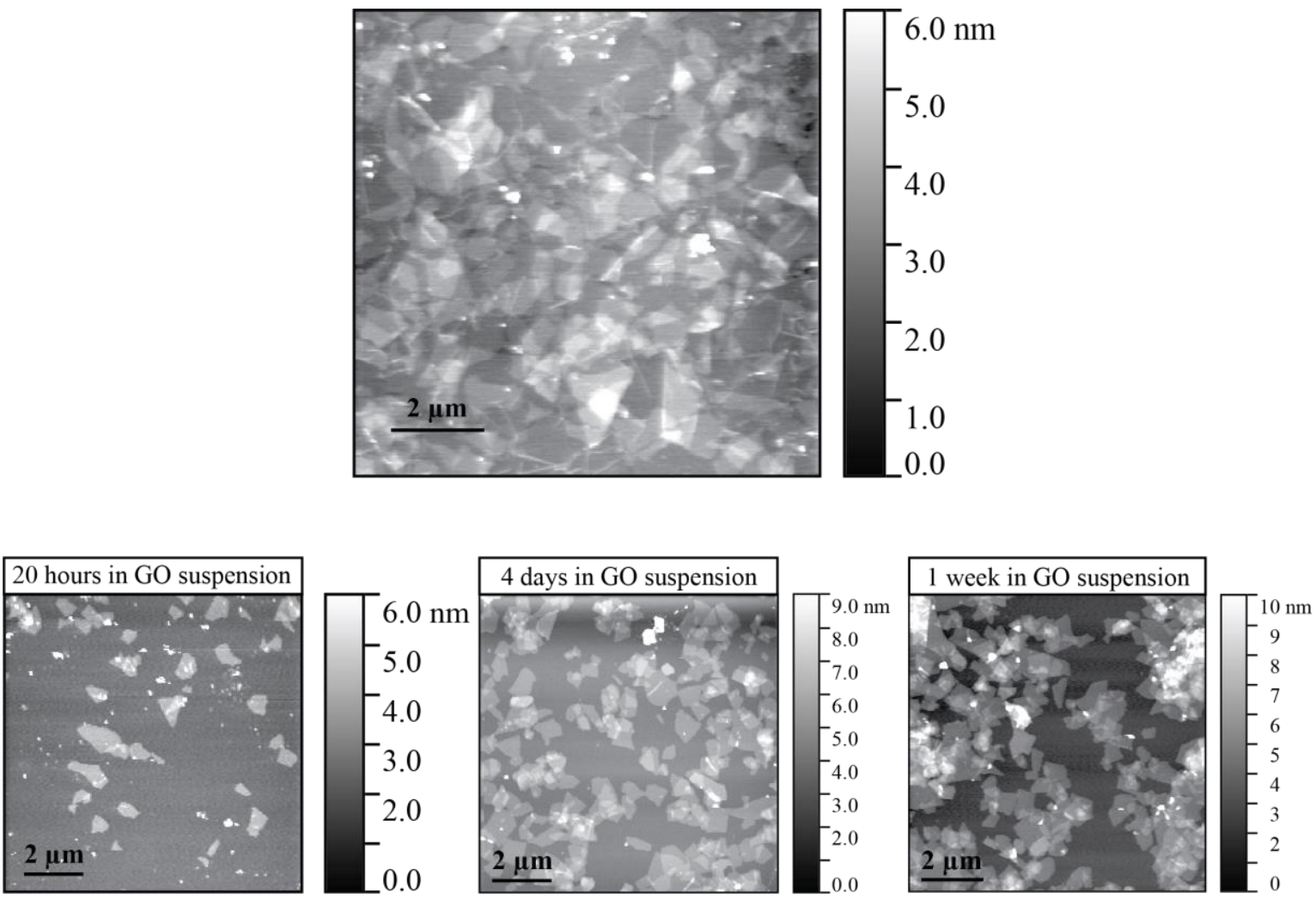

Figure 6.21 Atomic force micrographs of self-assembled GO films on silicon wafers. (Top) A silicon wafer that had been functionalized with APTES and then immersed in a methanolic suspension of $\mathrm{GO}$ at $70^{\circ} \mathrm{C}$ for two hours. (Bottom) Silicon wafers that had been functionalized with TEOS and then immersed in a methanolic suspension of GO at room temperature for 20 hours, 4 days, and 1 week. 
Future work. From these data it is apparent that a full (or nearly full) coverage GO thin film can be obtained on an APTES-functionalized glass surface at elevated temperatures but not on a TEOS-functionalized surface at room temperature. Further experiments should be conducted to determine whether GO can be bound to APTES-functionalized surfaces at lower temperatures or whether a more uniform thin film can be formed on TEOS-functionalized surfaces at elevated temperatures. Once GO monolayers can be formed on glass surfaces, these films can be reduced to electronically conducting RGO. The pairing of electronically conductive, atomically thin films on insulating substrates makes these films viable options for the channel regions in field-effect transistors. Further FET experiments should be performed to extract and verify the film's electronic characteristics. Additionally, patterned conductive thin films are useful in the electronics and sensing industries. By masking portions of the glass substrate during functionalization, patterned GO thin films should be achievable. 


\section{Chapter 7. Summary and Conclusions}

This thesis describes efforts toward improving electrochemical energy conversion systems, specifically in terms of heterogeneous electrocatalytic solar energy conversion and stabilization of redox mediators for use as long-term energy storage materials. Photoelectrochemistry is a promising method for the conversion of solar energy to storable chemical fuels. In photoelectrochemical cells, sunlight absorbed by semiconductor electrodes immersed in an electrolyte solution causes electrons to flow between the semiconductors, which drives redox reactions in the electrolyte solution, thereby generating chemical fuels.

One major obstacle for implementing large scale photoelectrochemical energy conversion is high activation overpotentials for redox reactions occurring at electrode surfaces. Addition of an electrocatalyst can help mitigate these high overpotentials. Specifically, heterogeneous molecular electrocatalysis is a promising platform that has received comparatively little attention in photoelectrochemical systems. Molecular catalysts are advantageous over solid-state catalysts because they have well-defined active sites that can be more easily understood and controlled. Heterogenization of molecular electrocatalysts is desirable because it means less material waste than homogeneous systems, and it opens the door to catalysts that are insoluble in the electrolyte medium. Heterogenization of a molecular species can be achieved by physisorption to a surface using van der Waals forces or by covalent attachment through chemical bonds to the surface. Physisorption has the benefit of being cheap and facile but often has the disadvantage of low stability. Covalent attachment is generally more expensive and difficult to achieve but can have better stability.

Both physisorbed and covalent heterogeneous electrocatalytic systems have received considerable attention especially using carbon-based electrodes because carbon is amenable to modification by both methods. Heterogeneous photoelectrocatalytic systems, however, have received far less attention in part because of the high surface-specificity of surface attachment. The work in this thesis describes the use of reduced graphene oxide (RGO) thin films on electrode

surfaces to immobilize a molecular electrocatalyst. These films can be used in 
photoelectrocatalytic systems because they are both transparent and conductive, allowing light to penetrate to the underlying semiconductor and electrons to penetrate to the electrolyte interface. RGO thin films can be deposited on essentially any electrode surface, thereby allowing previously studied methods for small molecule immobilization on carbon surfaces to be applied to semiconductor electrodes in photoelectrochemical cells.

A novel method of fabricating thin RGO films is presented in Chapter 2 of this thesis. This method is novel in that it uses an outer-sphere chemical reductant to reduce GO films to high quality RGO films. Because of the gentleness of the method, these RGO films can be casted on a wide variety of substrates. The reducing solution (cobaltocene and an organic acid) are noncorrosive to substrates like reactive metals (e.g. indium) and flexible substrates like paper, demonstrating the applicability of this method to a wide range of substrate materials. This is highly beneficial for use in photoelectrochemical systems, considering the low stability of many semiconductor surfaces. Furthermore, these RGO films are shown to adsorb the molecular proton reduction electrocatalyst cobalt(III) bis(dichlorobenzenedithiolate) (1). The adsorption is assumed to be due to $\pi$-stacking interactions with the aromatic network of RGO; however, some XPS and XAS data suggest that additional interactions with the surface may occur, such as coordination to oxide groups. Further characterization using XAS, XANES, and EXAFS is necessary.

Retention of the adsorbed catalyst on these RGO films is shown to be highly dependent on the surface morphology of the RGO. Under turnover conditions, the catalyst is quickly lost from smooth RGO films, whereas it is retained for significantly longer times on roughened films. Based on these results and published reports on graphite electrodes and RGO electrodeposited on FTOcoated glass electrodes, ${ }^{69,217-218}$ it is hypothesized that the catalyst can intercalate within rougher films, thereby becoming mechanically trapped. These data are relevant for research on adsorption of small molecules to graphitic surfaces using $\pi$-stacking interactions. The work here shows that while a molecule may readily adsorb to a surface, the interactions holding it on that surface may not be strong enough to retain it during a chemical transformation, such as catalytic turnover. However, if intercalation within the graphitic material also occurs, this can help to retain the molecule for a much longer period of time. This phenomenon can help explain variations and irreproducibility in reports of molecular adsorption to graphitic surfaces where different deposition methods and different substrates can produce very different film morphologies. Furthermore, it points toward potential approaches for improving stability of physisorbed systems that utilize $\pi$ - 
stacking interactions. For example, the strength of $\pi$-stacking interactions between a molecule and a surface may be improved by adding more $\pi$-systems or larger $\pi$-systems to the small molecule. For instance, the use of pyrene ligands rather than benzene ligands may enhance surface stability in the case of $\mathbf{1}$. Another option may be to fabricate reproducibly rough RGO films. If the roughness (and surface area) of these films can be defined and controlled and if they are thin enough to allow light transmittance, then they can be useful for studying heterogeneous molecular electrocatalysis in photoelectrochemical cells.

Besides the use of rough RGO surfaces, another method to improve catalyst retention developed here is the direct embedding of the electrocatalyst $\mathbf{1}$ in RGO films by co-deposition. This is achieved by dropcasting a mixture of a solution of $\mathbf{1}$ with a suspension of GO followed by reduction. This thesis further presents work describing kinetic limiting factors on electrocatalytic proton reduction in these electrocatalytic "mixed" films. By varying the thickness of the films and the density of catalyst within the films, it is shown that the electrocatalytic current is limited by a convolution of factors, most likely diffusion of product and substrate within the film as well as electrical conductivity of the film. For thinner films, the current appears to be limited by diffusion of either substrate or product (or both), whereas for thicker films, another limiting factor comes into play, most likely electrical conductivity of the RGO films. This thesis demonstrates the first work on understanding the kinetics of electrocatalysis in embedded RGO films. To date, literature has been published on the kinetics of proton reduction in electrocatalytic polymer films ${ }^{239}$, 241-242 and other reactions in electrocatalytic inorganic films (such as porous metal oxides). ${ }^{232,236,346}$ Given the unique structure of RGO compared to conventional polymers and inorganic films, we expect the electrical conductivity and diffusion of material into and out of the film to be similarly unique. Further experiments on this front are described in Chapter 4, which will help to identify whether product or substrate diffusion is limiting in thinner films and to determine whether changes in morphology influence the attainable current in thick films versus thin films. For example, determining catalytic reversibility or whether the catalyst can be poisoned by product can help determine whether product buildup can affect the system. Statistical imaging of the films can help demonstrate whether the surface morphology is consistent over a range of thicknesses.

In addition to the experiments on physisorbed $\mathbf{1}$ on and within RGO thin films on electrode surfaces, some data on method development for covalent attachment of molecules to RGO films are also presented. The following three methods for attachment of small molecules on RGO are 
demonstrated: attachment of amine-containing molecules via RGO epoxide groups; azidification of RGO followed by a copper-catalyzed click reaction with an alkyne-containing species; and attachment of an alkoxysilane molecule to RGO alcohol groups. Each of these methods successfully grafts a small molecule to the surface of graphene oxide thin films. Reaction with silanes appears to give the highest surface coverage and the click reaction appears to give the lowest surface coverage. Further experiments to optimize each of these grafting reactions are detailed in Chapter 6. Ultimately, these reactions will be used to graft a version of 1 to RGO thin films. Current work is focused on synthesizing derivatives of $\mathbf{1}$ containing amine, alkyne, and alkoxysilane functionalities. Once these derivatives are synthesized, RGO films containing the three types of grafted catalyst will be compared to each other and to the physisorbed systems. This work will demonstrate differences and similarities in the electrochemistry, catalytic activity, and stability of different methods of immobilizing 1 on graphitic carbon electrode surfaces.

The ultimate goal of developing these RGO films with immobilized catalyst is to use them on semiconductor electrodes for photoelectrocatalytic proton reduction. Some preliminary results are shown in Chapter 6 of this thesis using these RGO films to modify gallium phosphide photocathodes. Both surface-adsorbed $\mathbf{1}$ on smooth RGO films and RGO films embedded with $\mathbf{1}$ were deposited on $\mathrm{p}$-GaP surfaces. The data from these experiments are not wholly conclusive but suggest that inconsistencies in RGO film deposition lead to large variations in photocurrent and onset potential. Further experiments are presented that suggest modifications of the GaP surface that will protect the native surface against chemical attack and allow for spincasting of highly uniform and reproducible RGO films. Additionally, other types of semiconductors should be tested with these RGO thin films, for example $\mathrm{Si}$, which has a very large overpotential for proton reduction.

Chapter 6 also presents some preliminary data that uses RGO thin films to address another shortcoming in photoelectrochemical energy conversion. Many semiconductor materials exhibit low stability in water and under illumination and applied potential. Silicon is one such material. Under illumination and positive bias in water, $\mathrm{n}-\mathrm{Si}$ rapidly oxidizes to $\mathrm{SiO}_{\mathrm{x}}$ leading to loss in photocurrent and photovoltage. In basic conditions (commonly used in water oxidation) corrosion also occurs because $\mathrm{SiO}_{\mathrm{x}}$ is soluble in basic media. Methods for protecting semiconductor surfaces against degradation pathways like this involve chemical passivation by saturating dangling bonds at the surface, which lowers the energy of the surface, or physical passivation by depositing a layer 
that can block reactive species from approaching the semiconductor surface. Many of these methods, however, are highly surface-specific and expensive to implement.

Reported here are data demonstrating the ability of RGO thin films, which are cheap and not surface-specific, to passivate n-Si surfaces. Silicon (111) surfaces were first chemically passivated with a benzyl-alcohol group and then RGO thin films were spincasted on top. Photoelectrochemical and XPS data demonstrate longer-lived photocurrent and less silicon oxidation in pH 7 electrolyte on electrodes with the RGO film compared to electrodes without the RGO film, suggesting RGO can act as a barrier for reactive species such as $\mathrm{OH}^{-}$and $\mathrm{H}_{2} \mathrm{O}$ to approach the silicon surface. Further experiments should first demonstrate reproducibility of the data presented here. Then the ability of RGO to passivate other semiconductor surfaces (such as $\mathrm{GaP}$ ) and in other conditions (such as acidic or basic media) should be investigated. An important benefit of using RGO to passivate semiconductor photoelectrodes is that RGO can simultaneously be used to immobilize electrocatalysts, thereby addressing two problems associated with photoelectrochemical cells. Future experiments should investigate the ability of RGO thin films to simultaneously protect photoelectrode surfaces and facilitate heterogeneous electrocatalysis by immobilizing a catalyst like $\mathbf{1}$.

Chapter 5 of this thesis addresses a different area in photoelectrochemical energy storage. While the water-splitting reaction is the most common reaction in fuel-generating photoelectrochemical cells, hydrogen gas presents many safety concerns when it comes to storage and transportation because of its gaseous form and reactivity with oxygen. Solid-state, liquid, and dissolved redox couples do not present these safety concerns and are therefore more desirable than $\mathrm{H}_{2}$ as fuel sources in this respect. Viologens, for example, are a class of organic molecules that are redox active and are commonly used in solution state or as polymers in electrochemical energy storage. One problem that arises when using viologens to store electrochemical energy is their sensitivity to oxygen. Viologen radicals readily oxidize in trace amounts of oxygen, which limits their shelf-life.

Chapter 5 addresses this issue by describing the use of cucurbituril cage molecules to trap and stabilize a unique oxidation state of bis-viologens. Free in solution, these viologens undergo a 2 -electron reduction, between the 4+ (stable in air) and the 2+ oxidation states. When encapsulated within $\mathrm{CB}[8]$, an intermediate oxidation state $(3+)$ is accessible. Interestingly, these entrapped $3+$ bis-viologens are exceptionally stable in the presence of oxygen. Oxidation of solutions of the 
encapsulated 3+ viologens exposed to air were about an order of magnitude slower than oxidation of solutions of the free $2+$ viologens exposed to air. Molecular orbital calculations on these species suggest that the origin of this stability arises from a SOMO orbital that is embedded between the two aromatic moieties of the folded viologen within the cucurbituril. This phenomenon occurs because the viologen moieties are forced into a unique conformation by a combination of their covalent linkage and encapsulation within the cage molecule.

This work demonstrates a method for stabilizing a reduced viologen in the presence of oxygen by using a combination of synthetic and supramolecular techniques. Understanding the basis of this stabilization opens the door for development of other stabilized systems. For example, the viologen moieties can be methylated to provide a larger tunneling barrier around the buried SOMO electron, potentially slowing reaction with $\mathrm{O}_{2}$ even further. Alternatively, a solid porous structure, like a MOF, could potentially be designed with cavities that can host bis-viologens in the same way that $\mathrm{CB}[8]$ does in solution. In this way, the viologens will be stabilized both by forced conformation (as in $\mathrm{CB}[8]$ ) and by residing inside a solid support into which diffusion of $\mathrm{O}_{2}$ should be slow. Looking in another direction, solubility of $\mathrm{CB}[8]$ in water is quite poor. Development of an alternative cage molecule that has the same effect on bis-viologens but is also highly soluble in water would be extremely useful. And lastly, development of a method to crystallize 3+ bis-viologens entrapped in $\mathrm{CB}$ [8] may also be useful, as a way to store the charged material less densely.

In summary, this thesis presents important progress in two areas related to photoelectrochemical energy conversion and storage. The first is immobilization of a molecular electrocatalyst on both electrode and photoelectrode surfaces, which has previously proved difficult due in part to the surface-specificity of immobilization techniques. In this thesis, immobilization is achieved by use of transparent and conductive graphitic thin films that allow for both physisorption and covalent attachment of molecular species. Insights into the role of surface morphology of these films on the retention of a physisorbed molecular catalyst as well as limiting kinetic factors for electrocatalysis using these films are described. This work presents a cheap, facile, and generalizable method for immobilizing molecular electrocatalysts on electrode and semiconductor surfaces and describes the first efforts in understanding the fundamental kinetics of an electrocatalytic graphitic film. Second, stabilization of organic radicals for long-term storage of electrochemical energy is presented. Viologens possess many characteristics beneficial for use as 
long-term energy storage materials but suffer from fast oxidation in the presence of trace oxygen. Synergistic effects from covalent linkage and supramolecular assembly are demonstrated in this thesis to thermodynamically and kinetically stabilize two reduced viologen radicals against reaction with oxygen. This work presents a facile method that allows for extending the shelf-life of reduced viologen radicals and points towards methods for improving this shelf-life by further slowing down the reaction rate with oxygen. 


\section{Bibliography}

1. Berna, F.; Goldberg, P.; Horwitz, L. K.; Brink, J.; Holt, S.; Bamford, M.; Chazan, M., Microstratigraphic evidence of in situ fire in the Acheulean strata of Wonderwerk Cave, Northern Cape province, South Africa. Proceedings of the National Academy of Sciences 2012, 109 (20), E1215-E1220.

2. DeLong, J. P.; Burger, O., Socio-Economic Instability and the Scaling of Energy Use with Population Size. PLOS ONE 2015, 10 (6), e0130547.

3. Capuano, L., International Energy Outlook 2018. US Energy Information Administration, C. f. S. a. I. S., Ed. Washington DC, 2018.

4. Marchal, V.; Dellink, R.; Vuuren, D. v.; Clapp, C.; Chateau, J.; Lanzi, E.; Magne, B.; Vliet, J. v. OECD Environmental Outlook to 2050; OECD: 2011.

5. Wuebbles, D. J.; Fahey, D. W.; Hibbard, K. A.; DeAngelo, B.; Doherty, S.; Hayhoe, K.; Horton, R.; Kossin, J. P.; Taylor, P. C.; Waple, A. M.; Weaver, C. P. 2017: Executive summary. In: Climate Science Special Report: Fourth National Climate Assessment, Volume I; U.S. Global Change Research Program: Washington, DC, USA, 2017.

6. Levy, B. S.; Patz, J. A., Climate Change, Human Rights, and Social Justice. Annals of Global Health 2015, 81 (3), 310-322.

7. Patz, J. A.; Gibbs, H. K.; Foley, J. A.; Rogers, J. V.; Smith, K. R., Climate Change and Global Health: Quantifying a Growing Ethical Crisis. EcoHealth 2007, 4 (4), 397-405.

8. Tol, R. S. J., The Economic Impacts of Climate Change. Review of Environmental Economics and Policy 2018, 12 (1), 4-25.

9. Lewis, N. S.; Nocera, D. G., Powering the planet: Chemical challenges in solar energy utilization. Proceedings of the National Academy of Sciences 2006, 103 (43), 15729-15735.

10. Ismail, A. A.; Bahnemann, D. W., Photochemical splitting of water for hydrogen production by photocatalysis: A review. Solar Energy Materials and Solar Cells 2014, 128, 85-101.

11. Andreiadis, E. S.; Chavarot-Kerlidou, M.; Fontecave, M.; Artero, V., Artificial Photosynthesis: From Molecular Catalysts for Light-driven Water Splitting to Photoelectrochemical Cells. Photochemistry and Photobiology 2011, 87 (5), 946-964.

12. Ran, J.; Zhang, J.; Yu, J.; Jaroniec, M.; Qiao, S. Z., Earth-abundant cocatalysts for semiconductor-based photocatalytic water splitting. Chemical Society Reviews 2014, 43 (22), 7787-7812.

13. Fonash, S., Solar cell device physics. Elsevier: 2012.

14. Luo, J.; Im, J.-H.; Mayer, M. T.; Schreier, M.; Nazeeruddin, M. K.; Park, N.-G.; Tilley, S. D.; Fan, H. J.; Grätzel, M., Water photolysis at $12.3 \%$ efficiency via perovskite photovoltaics and Earth-abundant catalysts. Science 2014, 345 (6204), 1593-1596.

15. Gao, Y.; Le Corre, V. M.; Gaïtis, A.; Neophytou, M.; Hamid, M. A.; Takanabe, K.; Beaujuge, P. M., Homo-Tandem Polymer Solar Cells with VOC> 1.8 V for Efficient PV-Driven Water Splitting. Advanced Materials 2016, 28 (17), 3366-3373. 
16. Li, W.; Jiang, N.; Hu, B.; Liu, X.; Song, F.; Han, G.; Jordan, T. J.; Hanson, T. B.; Liu, T. L.; Sun, Y., Electrolyzer design for flexible decoupled water splitting and organic upgrading with electron reservoirs. Chem 2018, 4 (3), 637-649.

17. Jia, J.; Seitz, L. C.; Benck, J. D.; Huo, Y.; Chen, Y.; Ng, J. W. D.; Bilir, T.; Harris, J. S.; Jaramillo, T. F., Solar water splitting by photovoltaic-electrolysis with a solar-to-hydrogen efficiency over 30\%. Nature communications 2016, 7 (1), 1-6.

18. Sankir, N. D.; Sankir, M., Photoelectrochemical Solar Cells. John Wiley \& Sons: 2018.

19. Li, Z.; Luo, W.; Zhang, M.; Feng, J.; Zou, Z., Photoelectrochemical cells for solar hydrogen production: current state of promising photoelectrodes, methods to improve their properties, and outlook. Energy \& Environmental Science 2013, 6 (2), 347-370.

20. Memming, R., Photoelectrochemical solar energy conversion. In Electrochemistry II, Springer: 1988; pp 79-112.

21. Tan, M. X.; Laibinis, P. E.; Nguyen, S. T.; Kesselman, J. M.; Stanton, C. E.; Lewis, N. S., Principles and applications of semiconductor photoelectrochemistry. Progress in inorganic chemistry 1994, 21-144.

22. Abe, R., Recent progress on photocatalytic and photoelectrochemical water splitting under visible light irradiation. Journal of Photochemistry and Photobiology C: Photochemistry Reviews 2010, 11 (4), 179-209.

23. Dutta, S., A review on production, storage of hydrogen and its utilization as an energy resource. Journal of Industrial and Engineering Chemistry 2014, 20 (4), 1148-1156.

24. Ye, R.; Henkensmeier, D.; Yoon, S. J.; Huang, Z.; Kim, D. K.; Chang, Z.; Kim, S.; Chen, R., Redox Flow Batteries for Energy Storage: A Technology Review. Journal of Electrochemical Energy Conversion and Storage 2017, 15 (1).

25. Singh, V.; Kim, S.; Kang, J.; Byon, H. R., Aqueous organic redox flow batteries. Nano Research 2019, 12 (9), 1988-2001.

26. Selzer, Y.; Turyan, I.; Mandler, D., Studying heterogeneous catalysis by the scanning electrochemical microscope (SECM): The reduction of protons by methyl viologen catalyzed by a platinum surface. The Journal of Physical Chemistry B 1999, 103 (9), 1509-1517.

27. Tilley, S. D.; Lany, S.; Van de Krol, R., Advances in Photoelectrochemical Water Splitting: Theory, Experiment and Systems Analysis. Royal Society of Chemistry: 2018.

28. Mills, A.; Douglas, P.; Russell, T., Kinetic study of the reduction of water to hydrogen by reduced methyl viologen mediated by platinised alumina. Journal of the Chemical Society, Faraday Transactions 1990, 86 (9), 1417-1423.

29. Agar, J.; Bowden, F. P., The kinetics of electrode reactions. I and II. Proceedings of the Royal Society of London. Series A. Mathematical and Physical Sciences 1938, 169 (937), 206-234.

30. Bard, A. J.; Faulkner, L. R., Electrochemical Methods: Fundamental and Applications. 2nd ed.; John Wiley \& Sons: Hoboken, NJ, 2001.

31. Conway, B.; Jerkiewicz, G., Relation of energies and coverages of underpotential and overpotential deposited $\mathrm{H}$ at $\mathrm{Pt}$ and other metals to the 'volcano curve'for cathodic $\mathrm{H} 2$ evolution kinetics. Electrochimica Acta 2000, 45 (25-26), 4075-4083.

32. McKone, J. R.; Marinescu, S. C.; Brunschwig, B. S.; Winkler, J. R.; Gray, H. B., Earthabundant hydrogen evolution electrocatalysts. Chemical Science 2014, 5 (3), 865-878.

33. Tran, P. D.; Artero, V.; Fontecave, M., Water electrolysis and photoelectrolysis on electrodes engineered using biological and bio-inspired molecular systems. Energy \& Environmental Science 2010, 3 (6), 727-747. 
34. Bullock, R. M.; Das, A. K.; Appel, A. M., Surface immobilization of molecular electrocatalysts for energy conversion. Chemistry-A European Journal 2017, 23 (32), 76267641.

35. Tian, H., Molecular Catalyst Immobilized Photocathodes for Water/Proton and Carbon Dioxide Reduction. ChemSusChem 2015, 8 (22), 3746-3759.

36. Yan, Y.; Xia, B. Y.; Zhao, B.; Wang, X., A review on noble-metal-free bifunctional heterogeneous catalysts for overall electrochemical water splitting. Journal of Materials Chemistry A 2016, 4 (45), 17587-17603.

37. Zou, X.; Zhang, Y., Noble metal-free hydrogen evolution catalysts for water splitting. Chemical Society Reviews 2015, 44 (15), 5148-5180.

38. Zeng, M.; Li, Y., Recent advances in heterogeneous electrocatalysts for the hydrogen evolution reaction. Journal of Materials Chemistry A 2015, 3 (29), 14942-14962.

39. Bansal, A.; Li, X.; Lauermann, I.; Lewis, N. S.; Yi, S. I.; Weinberg, W. H., Alkylation of Si Surfaces Using a Two-Step Halogenation/Grignard Route. Journal of the American Chemical Society 1996, 118 (30), 7225-7226.

40. Schvartzman, M.; Sidorov, V.; Ritter, D.; Paz, Y., Surface passivation of (100) InP by organic thiols and polyimide as characterized by steady-state photoluminescence. Semiconductor science and technology 2001, 16 (10), L68.

41. Huang, M. L.; Chang, Y. C.; Chang, C. H.; Lee, Y. J.; Chang, P.; Kwo, J.; Wu, T. B.; Hong, M., Surface passivation of III-V compound semiconductors using atomic-layer-depositiongrown Al2O3. Applied Physics Letters 2005, 87 (25), 252104.

42. Yang, G. H.; Zhang, Y.; Kang, E. T.; Neoh, K. G.; Huang, W.; Teng, J. H., Surface Passivation of (100)-Oriented GaAs via Plasma Deposition of an Ultrathin S-Containing Polymer Film and Its Effect on Photoluminescence. The Journal of Physical Chemistry B 2003, 107 (33), 8592-8598.

43. Nielander, A. C.; Bierman, M. J.; Petrone, N.; Strandwitz, N. C.; Ardo, S.; Yang, F.; Hone, J.; Lewis, N. S., Photoelectrochemical Behavior of n-Type Si(111) Electrodes Coated With a Single Layer of Graphene. Journal of the American Chemical Society 2013, 135 (46), 1724617249.

44. Wang, W.; Xu, X.; Zhou, W.; Shao, Z., Recent Progress in Metal-Organic Frameworks for Applications in Electrocatalytic and Photocatalytic Water Splitting. Advanced Science 2017, 4 (4), 1600371.

45. Hou, Y.; Zhuang, X.; Feng, X., Recent Advances in Earth-Abundant Heterogeneous Electrocatalysts for Photoelectrochemical Water Splitting. Small Methods 2017, 1 (6), 1700090.

46. Thoi, V. S.; Sun, Y.; Long, J. R.; Chang, C. J., Complexes of earth-abundant metals for catalytic electrochemical hydrogen generation under aqueous conditions. Chemical Society Reviews 2013, 42 (6), 2388-2400.

47. Wang, M.; Li, F., Electrochemical Water Oxidation and Reduction Catalyzed by Organometallic Compounds. In Applied Homogeneous Catalysis with Organometallic Compounds, 2017; pp 1135-1194.

48. Wang, N.; Zheng, H.; Zhang, W.; Cao, R., Mononuclear first-row transition-metal complexes as molecular catalysts for water oxidation. Chinese Journal of Catalysis 2018, 39 (2), 228244. 
49. Joya, K. S.; Vallés-Pardo, J. L.; Joya, Y. F.; Eisenmayer, T.; Thomas, B.; Buda, F.; de Groot, H. J. M., Molecular Catalytic Assemblies for Electrodriven Water Splitting. ChemPlusChem 2013, 78 (1), 35-47.

50. Wang, M.; Han, K.; Zhang, S.; Sun, L., Integration of organometallic complexes with semiconductors and other nanomaterials for photocatalytic $\mathrm{H} 2$ production. Coordination Chemistry Reviews 2015, 287, 1-14.

51. Coutard, N.; Kaeffer, N.; Artero, V., Molecular engineered nanomaterials for catalytic hydrogen evolution and oxidation. Chemical Communications 2016, 52 (95), 13728-13748.

52. Materna, K. L.; Crabtree, R. H.; Brudvig, G. W., Anchoring groups for photocatalytic water oxidation on metal oxide surfaces. Chemical Society Reviews 2017, 46 (20), 6099-6110.

53. Silverman, B. M.; Wieghaus, K. A.; Schwartz, J., Comparative properties of siloxane vs phosphonate monolayers on a key titanium alloy. Langmuir 2005, 21 (1), 225-228.

54. Campisciano, V.; Gruttadauria, M.; Giacalone, F., Modified Nanocarbons for Catalysis. ChemCatChem 2019, 11 (1), 90-133.

55. Kuila, T.; Bose, S.; Mishra, A. K.; Khanra, P.; Kim, N. H.; Lee, J. H., Chemical functionalization of graphene and its applications. Progress in Materials Science 2012, 57 (7), 1061-1105.

56. Toma, F. M.; Sartorel, A.; Iurlo, M.; Carraro, M.; Parisse, P.; Maccato, C.; Rapino, S.; Gonzalez, B. R.; Amenitsch, H.; Da Ros, T.; Casalis, L.; Goldoni, A.; Marcaccio, M.; Scorrano, G.; Scoles, G.; Paolucci, F.; Prato, M.; Bonchio, M., Efficient water oxidation at carbon nanotube-polyoxometalate electrocatalytic interfaces. Nature Chemistry 2010, 2 (10), 826-831.

57. Sandanayaka, A. S. D.; Chitta, R.; Subbaiyan, N. K.; D’Souza, L.; Ito, O.; D’Souza, F., Photoinduced Charge Separation in Ion-Paired Porphyrin-Single-Wall Carbon Nanotube Donor-Acceptor Hybrids. The Journal of Physical Chemistry C 2009, 113 (30), 1342513432.

58. Wang, R.-Q.; Suo, L.; Zheng, D.-M.; Du, Y.; Wu, L.-X.; Bi, L.-H., A heterogeneous catalyst containing tetraruthenium (IV)-substituted silicotungstate: Preparation, characterization and catalytic performance toward oxidation of n-tetradecane with air. Inorganica Chimica Acta 2016, 443, 218-223.

59. Le Goff, A.; Artero, V.; Jousselme, B.; Tran, P. D.; Guillet, N.; Métayé, R.; Fihri, A.; Palacin, S.; Fontecave, M., From hydrogenases to noble metal-free catalytic nanomaterials for H2 production and uptake. Science 2009, 326 (5958), 1384-1387.

60. Tran, P. D.; Le Goff, A.; Heidkamp, J.; Jousselme, B.; Guillet, N.; Palacin, S.; Dau, H.; Fontecave, M.; Artero, V., Noncovalent Modification of Carbon Nanotubes with PyreneFunctionalized Nickel Complexes: Carbon Monoxide Tolerant Catalysts for Hydrogen Evolution and Uptake. Angewandte Chemie International Edition 2011, 50 (6), 1371-1374.

61. Blakemore, J. D.; Gupta, A.; Warren, J. J.; Brunschwig, B. S.; Gray, H. B., Noncovalent immobilization of electrocatalysts on carbon electrodes for fuel production. Journal of the American Chemical Society 2013, 135 (49), 18288-18291.

62. Downes, C. A.; Marinescu, S. C., Efficient electrochemical and photoelectrochemical H2 production from water by a cobalt dithiolene one-dimensional metal-organic surface. Journal of the American Chemical Society 2015, 137 (43), 13740-13743.

63. Andreiadis, E. S.; Jacques, P.-A.; Tran, P. D.; Leyris, A.; Chavarot-Kerlidou, M.; Jousselme, B.; Matheron, M.; Pécaut, J.; Palacin, S.; Fontecave, M., Molecular engineering of a cobalt- 
based electrocatalytic nanomaterial for $\mathrm{H} 2$ evolution under fully aqueous conditions. Nature chemistry 2013, 5 (1), 48-53.

64. Muresan, N. M.; Willkomm, J.; Mersch, D.; Vaynzof, Y.; Reisner, E., Immobilization of a molecular cobaloxime catalyst for hydrogen evolution on a mesoporous metal oxide electrode. Angewandte Chemie International Edition 2012, 51 (51), 12749-12753.

65. Thomas, C. M.; Rüdiger, O.; Liu, T.; Carson, C. E.; Hall, M. B.; Darensbourg, M. Y., Synthesis of carboxylic acid-modified [FeFe]-hydrogenase model complexes amenable to surface immobilization. Organometallics 2007, 26 (16), 3976-3984.

66. Al Cheikh, J.; Villagra, A.; Ranjbari, A.; Pradon, A.; Antuch, M.; Dragoe, D.; Millet, P.; Assaud, L., Engineering a cobalt clathrochelate/glassy carbon interface for the hydrogen evolution reaction. Applied Catalysis B: Environmental 2019, 250, 292-300.

67. Li, X.; Lei, H.; Liu, J.; Zhao, X.; Ding, S.; Zhang, Z.; Tao, X.; Zhang, W.; Wang, W.; Zheng, $\mathrm{X}$., Carbon Nanotubes with Cobalt Corroles for Hydrogen and Oxygen Evolution in $\mathrm{pH}$ 0-14 Solutions. Angewandte Chemie International Edition 2018, 57 (46), 15070-15075.

68. McAllister, J.; Bandeira, N. A.; McGlynn, J. C.; Ganin, A. Y.; Song, Y.-F.; Bo, C.; Miras, H. N., Tuning and mechanistic insights of metal chalcogenide molecular catalysts for the hydrogen-evolution reaction. Nature communications 2019, 10 (1), 1-10.

69. Eady, S. C.; MacInnes, M. M.; Lehnert, N., Immobilized Cobalt Bis (benzenedithiolate) Complexes: Exceptionally Active Heterogeneous Electrocatalysts for Dihydrogen Production from Mildly Acidic Aqueous Solutions. Inorganic chemistry 2017, 56 (19), 11654-11667.

70. Seo, J.; Pekarek, R. T.; Rose, M. J., Photoelectrochemical operation of a surface-bound, nickel-phosphine $\mathrm{H} 2$ evolution catalyst on p-Si (111): a molecular semiconductor| catalyst construct. Chemical Communications 2015, 51 (68), 13264-13267.

71. Hou, Y.; Abrams, B. L.; Vesborg, P. C.; Björketun, M. E.; Herbst, K.; Bech, L.; Setti, A. M.; Damsgaard, C. D.; Pedersen, T.; Hansen, O., Bioinspired molecular co-catalysts bonded to a silicon photocathode for solar hydrogen evolution. Nature materials 2011, 10 (6), 434-438.

72. Nann, T.; Ibrahim, S. K.; Woi, P. M.; Xu, S.; Ziegler, J.; Pickett, C. J., Water splitting by visible light: a nanophotocathode for hydrogen production. Angewandte Chemie International Edition 2010, 49 (9), 1574-1577.

73. Beiler, A. M.; Khusnutdinova, D.; Jacob, S. I.; Moore, G. F., Chemistry at the interface: polymer-functionalized $\mathrm{GaP}$ semiconductors for solar hydrogen production. Industrial \& Engineering Chemistry Research 2016, 55 (18), 5306-5314.

74. Beden, B.; Leger, J., C. Lamy in J. O'M. Bockris, BE Conway and RE White (Eds.), Modern Aspects of Electrochemistry, Vol. 22. Plenum Press, New York: 1992.

75. Memming, R., Semiconductor electrochemistry. John Wiley \& Sons: 2015.

76. Sim, U.; Yang, T.-Y.; Moon, J.; An, J.; Hwang, J.; Seo, J.-H.; Lee, J.; Kim, K. Y.; Lee, J.; Han, S., N-doped monolayer graphene catalyst on silicon photocathode for hydrogen production. Energy \& Environmental Science 2013, 6 (12), 3658-3664.

77. Chatterjee, S.; Carter, R.; Oakes, L.; Erwin, W. R.; Bardhan, R.; Pint, C. L., Electrochemical and corrosion stability of nanostructured silicon by graphene coatings: toward high power porous silicon supercapacitors. The Journal of Physical Chemistry C 2014, 118 (20), 1089310902.

78. Tan, R. K. L.; Reeves, S. P.; Hashemi, N.; Thomas, D. G.; Kavak, E.; Montazami, R.; Hashemi, N. N., Graphene as a flexible electrode: review of fabrication approaches. Journal of Materials Chemistry A 2017, 5 (34), 17777-17803. 
79. Choi, W.; Lahiri, I.; Seelaboyina, R.; Kang, Y. S., Synthesis of Graphene and Its Applications: A Review. Critical Reviews in Solid State and Materials Sciences 2010, 35 (1), 52-71.

80. Poh, H. L.; Šaněk, F.; Ambrosi, A.; Zhao, G.; Sofer, Z.; Pumera, M., Graphenes prepared by Staudenmaier, Hofmann and Hummers methods with consequent thermal exfoliation exhibit very different electrochemical properties. Nanoscale 2012, 4 (11), 3515-3522.

81. Cote, L. J.; Kim, F.; Huang, J., Langmuir-Blodgett Assembly of Graphite Oxide Single Layers. Journal of the American Chemical Society 2009, 131 (3), 1043-1049.

82. Wang, J.; Liang, M.; Fang, Y.; Qiu, T.; Zhang, J.; Zhi, L., Rod-Coating: Towards Large-Area Fabrication of Uniform Reduced Graphene Oxide Films for Flexible Touch Screens. Advanced Materials 2012, 24 (21), 2874-2878.

83. Eda, G.; Chhowalla, M., Graphene-based Composite Thin Films for Electronics. Nano Letters 2009, 9 (2), 814-818.

84. Eluyemi, M.; Eleruja, M.; Adedeji, A.; Olofinjana, B.; Fasakin, O.; Akinwunmi, O.; Ilori, O.; Famojuro, A.; Ayinde, S.; Ajayi, E., Synthesis and characterization of graphene oxide and reduced graphene oxide thin films deposited by spray pyrolysis method. Graphene 2016, 5 (3), 143-154.

85. Bao, H.; Pan, Y.; Ping, Y.; Sahoo, N. G.; Wu, T.; Li, L.; Li, J.; Gan, L. H., Chitosanfunctionalized graphene oxide as a nanocarrier for drug and gene delivery. Small 2011, 7 (11), 1569-1578.

86. Niyogi, S.; Bekyarova, E.; Itkis, M. E.; McWilliams, J. L.; Hamon, M. A.; Haddon, R. C., Solution properties of graphite and graphene. Journal of the American Chemical Society 2006, 128 (24), 7720-7721.

87. Xu, Y.; Liu, Z.; Zhang, X.; Wang, Y.; Tian, J.; Huang, Y.; Ma, Y.; Zhang, X.; Chen, Y., A graphene hybrid material covalently functionalized with porphyrin: synthesis and optical limiting property. Advanced materials 2009, 21 (12), 1275-1279.

88. Jang, J.; Pham, V. H.; Hur, S. H.; Chung, J. S., Dispersibility of reduced alkylaminefunctionalized graphene oxides in organic solvents. Journal of colloid and interface science 2014, 424, 62-66.

89. Ikeda, Y.; Karim, M. R.; Takehira, H.; Matsui, T.; Taniguchi, T.; Koinuma, M.; Matsumoto, Y.; Hayami, S., Proton conductivity of graphene oxide hybrids with covalently functionalized alkylamines. Chemistry Letters 2013, 42 (11), 1412-1414.

90. Compton, O. C.; Dikin, D. A.; Putz, K. W.; Brinson, L. C.; Nguyen, S. T., Electrically conductive "alkylated" graphene paper via chemical reduction of amine-functionalized graphene oxide paper. Advanced materials 2010, 22 (8), 892-896.

91. Li, F.; Zhang, B.; Li, X.; Jiang, Y.; Chen, L.; Li, Y.; Sun, L., Highly efficient oxidation of water by a molecular catalyst immobilized on carbon nanotubes. Angewandte Chemie International Edition 2011, 50 (51), 12276-12279.

92. Hu, X. M.; Rønne, M. H.; Pedersen, S. U.; Skrydstrup, T.; Daasbjerg, K., Enhanced catalytic activity of cobalt porphyrin in $\mathrm{CO} 2$ electroreduction upon immobilization on carbon materials. Angewandte Chemie International Edition 2017, 56 (23), 6468-6472.

93. Hummers Jr, W. S.; Offeman, R. E., Preparation of graphitic oxide. Journal of the american chemical society 1958, 80 (6), 1339-1339.

94. Hofmann, U.; König, E., Untersuchungen über graphitoxyd. Zeitschrift für anorganische und allgemeine Chemie 1937, 234 (4), 311-336.

95. Staudenmaier, L., Verfahren zur darstellung der graphitsäure. Berichte der deutschen chemischen Gesellschaft 1898, 31 (2), 1481-1487. 
96. Chua, C. K.; Pumera, M., Chemical reduction of graphene oxide: a synthetic chemistry viewpoint. Chem. Soc. Rev. 2014, 43 (1), 291-312.

97. Pei, S.; Cheng, H.-M., The reduction of graphene oxide. Carbon 2012, 50 (9), 3210-3228.

98. Gao, W., The chemistry of graphene oxide. In Graphene oxide, Springer: 2015; pp 61-95.

99. Cheung, T., X-ray photoemission of polynuclear aromatic carbon. Journal of applied physics 1984, 55 (5), 1388-1393.

100. Leiro, J.; Heinonen, M.; Laiho, T.; Batirev, I., Core-level XPS spectra of fullerene, highly oriented pyrolitic graphite, and glassy carbon. Journal of Electron Spectroscopy and Related Phenomena 2003, 128 (2-3), 205-213.

101. Malard, L. M.; Pimenta, M. A.; Dresselhaus, G.; Dresselhaus, M. S., Raman spectroscopy in graphene. Physics Reports 2009, 473 (5-6), 51-87.

102. Pimenta, M.; Dresselhaus, G.; Dresselhaus, M. S.; Cancado, L.; Jorio, A.; Saito, R., Studying disorder in graphite-based systems by Raman spectroscopy. Physical chemistry chemical physics 2007, 9 (11), 1276-1290.

103. Tuinstra, F.; Koenig, J. L., Raman Spectrum of Graphite. J. Chem. Phys. 1970, 53 (3), 11261130.

104. Baranov, A.; Bekhterev, A.; Bobovich, Y. S.; Petrov, V., Interpretation of certain characteristics in Raman spectra of graphite and glassy carbon. Opt. Spektrosk 1987, 62 (1036), 99.

105. Cançado, L. G.; Takai, K.; Enoki, T.; Endo, M.; Kim, Y. A.; Mizusaki, H.; Jorio, A.; Coelho, L. N.; Magalhães-Paniago, R.; Pimenta, M. A., General equation for the determination of the crystallite size La of nanographite by Raman spectroscopy. Appl. Phys. Lett. 2006, 88 (16), 163106.

106. Ferrari, A. C.; Robertson, J., Interpretation of Raman spectra of disordered and amorphous carbon. Phys. Rev. B 2000, 61 (20), 14095-14107.

107. Lucchese, M. M.; Stavale, F.; Ferreira, E. H. M.; Vilani, C.; Moutinho, M. V. O.; Capaz, R. B.; Achete, C. A.; Jorio, A., Quantifying ion-induced defects and Raman relaxation length in graphene. Carbon 2010, 48 (5), 1592-1597.

108. Sadezky, A.; Muckenhuber, H.; Grothe, H.; Niessner, R.; Pöschl, U., Raman microspectroscopy of soot and related carbonaceous materials: Spectral analysis and structural information. Carbon 2005, 43 (8), 1731-1742.

109. Claramunt, S.; Varea, A.; López-Díaz, D.; Velázquez, M. M.; Cornet, A.; Cirera, A., The Importance of Interbands on the Interpretation of the Raman Spectrum of Graphene Oxide. $J$. Phys. Chem. C 2015, 119 (18), 10123-10129.

110. Kawashima, Y.; Katagiri, G., Fundamentals, overtones, and combinations in the Raman spectrum of graphite. Phys. Rev. B 1995, 52 (14), 10053-10059.

111. Tai, F.; Lee, S.-C.; Chen, J.; Wei, C.; Chang, S., Multipeak fitting analysis of Raman spectra on DLCH film. Journal of Raman Spectroscopy: An International Journal for Original Work in all Aspects of Raman Spectroscopy, Including Higher Order Processes, and also Brillouin and Rayleigh Scattering 2009, 40 (8), 1055-1059.

112. Kaniyoor, A.; Ramaprabhu, S., A Raman spectroscopic investigation of graphite oxide derived graphene. Aip Advances 2012, 2 (3), 032183.

113. Angoni, K., Remarks on the structure of carbon materials on the basis of raman spectra. Carbon 1993, 31 (4), 537-547. 
114. Cançado, L.; Reina, A.; Kong, J.; Dresselhaus, M., Geometrical approach for the study of G' band in the Raman spectrum of monolayer graphene, bilayer graphene, and bulk graphite. Physical Review B 2008, 77 (24), 245408.

115. Koutsos, V.; Manias, E.; Ten Brinke, G.; Hadziioannou, G., Atomic force microscopy and real atomic resolution. simple computer simulations. EPL (Europhysics Letters) 1994, 26 (2), 103.

116.Zhang, J.; Shen, G.; Wang, W.; Zhou, X.; Guo, S., Individual nanocomposite sheets of chemically reduced graphene oxide and poly (N-vinyl pyrrolidone): preparation and humidity sensing characteristics. Journal of Materials Chemistry 2010, 20 (48), 10824-10828.

117. Shaw, J. E.; Perumal, A.; Bradley, D. D.; Stavrinou, P. N.; Anthopoulos, T. D., Nanoscale current spreading analysis in solution-processed graphene oxide/silver nanowire transparent electrodes via conductive atomic force microscopy. Journal of Applied Physics 2016, 119 (19), 195501.

118. Shang, Y.; Zhang, D.; Liu, Y.; Guo, C., Preliminary comparison of different reduction methods of graphene oxide. Bulletin of Materials Science 2015, 38 (1), 7-12.

119. Eigler, S.; Dotzer, C.; Hirsch, A., Visualization of defect densities in reduced graphene oxide. Carbon 2012, 50 (10), 3666-3673.

120. Gao, W.; Alemany, L. B.; Ci, L.; Ajayan, P. M., New insights into the structure and reduction of graphite oxide. Nat. Chem. 2009, 1 (5), 403-8.

121. Pei, S.; Zhao, J.; Du, J.; Ren, W.; Cheng, H.-M., Direct reduction of graphene oxide films into highly conductive and flexible graphene films by hydrohalic acids. Carbon 2010, 48 (15), 4466-4474.

122.Fan, Z.-J.; Kai, W.; Yan, J.; Wei, T.; Zhi, L.-J.; Feng, J.; Ren, Y.-m.; Song, L.-P.; Wei, F., Facile synthesis of graphene nanosheets via Fe reduction of exfoliated graphite oxide. ACS nano 2011, 5 (1), 191-198.

123. Wang, Y.; Sun, L.; Fugetsu, B., Thiourea Dioxide as a Green Reductant for the Mass Production of Solution-Based Graphene. B. Chem. Soc. Jpn. 2012, 85 (12), 1339-1344.

124.Zhang, J.; Yang, H.; Shen, G.; Cheng, P.; Zhang, J.; Guo, S., Reduction of graphene oxide via L-ascorbic acid. Chem. Commun. 2010, 46 (7), 1112-1114.

125. Kumar, A.; Khandelwal, M., A novel synthesis of ultra thin graphene sheets for energy storage applications using malonic acid as a reducing agent. J. Mater. Chem. A 2014, 2 (47), 2034520357.

126. Moon, I. K.; Lee, J.; Ruoff, R. S.; Lee, H., Reduced graphene oxide by chemical graphitization. Nat. Commun. 2010, 1, 73.

127. Paredes, J.; Villar-Rodil, S.; Solís-Fernández, P.; Martínez-Alonso, A.; Tascon, J., Atomic force and scanning tunneling microscopy imaging of graphene nanosheets derived from graphite oxide. Langmuir 2009, 25 (10), 5957-5968.

128. Doğan, H. Ö.; Ekinci, D.; Demir, Ü., Atomic scale imaging and spectroscopic characterization of electrochemically reduced graphene oxide. Surface science 2013, 611, 54-59.

129. Rozada, R.; Paredes, J. I.; Villar-Rodil, S.; Martínez-Alonso, A.; Tascón, J. M., Towards full repair of defects in reduced graphene oxide films by two-step graphitization. Nano Research 2013, 6 (3), 216-233.

130. Dreyer, D. R.; Murali, S.; Zhu, Y.; Ruoff, R. S.; Bielawski, C. W., Reduction of graphite oxide using alcohols. J. Mater. Chem. 2011, 21 (10), 3443-3447.

131. Viinikanoja, A.; Kauppila, J.; Damlin, P.; Suominen, M.; Kvarnström, C., In situ FTIR and Raman spectroelectrochemical characterization of graphene oxide upon electrochemical 
reduction in organic solvents. Physical Chemistry Chemical Physics 2015, 17 (18), 1211512123.

132. Bagri, A.; Mattevi, C.; Acik, M.; Chabal, Y. J.; Chhowalla, M.; Shenoy, V. B., Structural evolution during the reduction of chemically derived graphene oxide. Nature chemistry $\mathbf{2 0 1 0}$, $2(7), 581-587$.

133. Storm, M. M.; Johnsen, R. E.; Norby, P., In situ X-ray powder diffraction studies of the synthesis of graphene oxide and formation of reduced graphene oxide. Journal of Solid State Chemistry 2016, 240, 49-54.

134. Pham, V. H.; Cuong, T. V.; Nguyen-Phan, T. D.; Pham, H. D.; Kim, E. J.; Hur, S. H.; Shin, E. W.; Kim, S.; Chung, J. S., One-step synthesis of superior dispersion of chemically converted graphene in organic solvents. Chem. Commun. 2010, 46 (24), 4375-7.

135. Pham, V. H.; Hur, S. H.; Kim, E. J.; Kim, B. S.; Chung, J. S., Highly efficient reduction of graphene oxide using ammonia borane. Chem. Commun. 2013, 49 (59), 6665-7.

136. Sliwa, W.; Bachowska, B.; Girek, T., Viologen-based supramolecular structures. Current Organic Chemistry 2012, 16 (10), 1332-1358.

137. Kaliappan, R.; Ling, Y.; Kaifer, A. E.; Ramamurthy, V., Sulfonatocalix[8]arene as a potential reaction cavity: photo- and electro-active dicationic guests arrest conformational equilibrium. Langmuir 2009, 25 (16), 8982-92.

138. Guo, D.-S.; Chen, S.; Qian, H.; Zhang, H.-Q.; Liu, Y., Electrochemical stimulus-responsive supramolecular polymer based on sulfonatocalixarene and viologen dimers. Chemical Communications 2010, 46 (15), 2620-2622.

139. Guo, D.-S.; Wang, L.-H.; Liu, Y., Highly effective binding of methyl viologen dication and its radical cation by p-sulfonatocalix $[4,5]$ arenes. The Journal of organic chemistry 2007, 72 (20), 7775-7778.

140. Yasuda, A.; Mori, H.; Seto, J., Electrochromic properties of alkylviologen-cyclodextrin systems. Journal of Applied Electrochemistry 1987, 17 (3), 567-573.

141. Yasuda, A.; Kondo, H.; Itabashi, M.; Seto, J., Structure changes of viologen+ $\beta$-cyclodextrin inclusion complex corresponding to the redox state of viologen. Journal of electroanalytical chemistry and interfacial electrochemistry 1986, 210 (2), 265-275.

142. Gadde, S.; E Kaifer, A., Cucurbituril complexes of redox active guests. Current Organic Chemistry 2011, 15 (1), 27-38.

143. Jeon, W. S.; Kim, H.-J.; Lee, C.; Kim, K., Control of the stoichiometry in host-guest complexation by redox chemistry of guests: Inclusion of methylviologen in cucurbit[8]uril. Chem. Commun. 2002, (17), 1828-1829.

144. Jariwala, D.; Sangwan, V. K.; Lauhon, L. J.; Marks, T. J.; Hersam, M. C., Carbon nanomaterials for electronics, optoelectronics, photovoltaics, and sensing. Chem. Soc. Rev. 2013, 42 (7), 2824-2860.

145.El-Kady, M. F.; Shao, Y.; Kaner, R. B., Graphene for batteries, supercapacitors and beyond. Nat. Rev. Mater. 2016, 1 (7), 1-14.

146. Wang, X.; Shi, G., An introduction to the chemistry of graphene. Phys. Chem. Chem. Phys. 2015, 17 (43), 28484-28504.

147.Zhou, M.; Wang, Y.; Zhai, Y.; Zhai, J.; Ren, W.; Wang, F.; Dong, S., Controlled synthesis of large-area and patterned electrochemically reduced graphene oxide films. Chem. Eur. J. 2009, 15 (25), 6116-20. 
148. Kauppila, J.; Kunnas, P.; Damlin, P.; Viinikanoja, A.; Kvarnström, C., Electrochemical reduction of graphene oxide films in aqueous and organic solutions. Electrochim. Acta 2013, 89, 84-89.

149. Bouša, M.; Frank, O.; Jirka, I.; Kavan, L., In situ Raman spectroelectrochemistry of graphene oxide. Phys. Status Solidi B 2013, 250 (12), 2662-2667.

150. Viinikanoja, A.; Kauppila, J.; Damlin, P.; Suominen, M.; Kvarnstrom, C., In situ FTIR and Raman spectroelectrochemical characterization of graphene oxide upon electrochemical reduction in organic solvents. Phys. Chem. Chem. Phys. 2015, 17 (18), 12115-23.

151. Toh, S. Y.; Loh, K. S.; Kamarudin, S. K.; Daud, W. R. W., Graphene production via electrochemical reduction of graphene oxide: Synthesis and characterisation. Chem. Eng. J. 2014, 251, 422-434.

152. Guo, H.-L.; Wang, X.-F.; Qian, Q.-Y.; Wang, F.-B.; Xia, X.-H., A green approach to the synthesis of graphene nanosheets. ACS Nano 2009, 9, 2653-2659.

153. Eady, S. C.; Peczonczyk, S. L.; Maldonado, S.; Lehnert, N., Facile heterogenization of a cobalt catalyst via graphene adsorption: robust and versatile dihydrogen production systems. Chem. Commun. 2014, 50 (59), 8065-8.

154. Alcantara, R.; Jimenez-Mateos, J. M.; Lavela, P.; Tirado, J. L., Carbon black: a promising electrode material for sodium-ion batteries. Electrochem. Commun. 2001, 3, 639-642.

155. Shao, Y.; Wang, J.; Engelhard, M.; Wang, C.; Lin, Y., Facile and controllable electrochemical reduction of graphene oxide and its applications. J. Mater. Chem. 2010, 20 (4), 743-748.

156. Wang, X.; Zhi, L.; Muellen, K., Transparent, Conductive Graphene Electrodes for DyeSensitized Solar Cells. Nano Lett. 2008, 8 (1), 323-327.

157. Becerril, H. A.; Mao, J.; Liu, Z.; Stoltenberg, R. M.; Bao, Z.; Chen, Y., Evaluation of Solution-Processed Reduced Graphene Oxide Films as Transparent Conductors. ACS Nano 2008, 2 (3), 463-470.

158. Qin, M.-M.; Ji, W.; Feng, Y.-Y.; Feng, W., Transparent conductive graphene films prepared by hydroiodic acid and thermal reduction. Chinese Phys. B 2014, 23 (2), 028103.

159. Chen, W.; Yan, L.; Bangal, P. R., Preparation of graphene by the rapid and mild thermal reduction of graphene oxide induced by microwaves. Carbon 2010, 48 (4), 1146-1152.

160. Acik, M.; Lee, G.; Mattevi, C.; Pirkle, A.; Wallace, R. M.; Chhowalla, M.; Cho, K.; Chabal, Y., The Role of Oxygen during Thermal Reduction of Graphene Oxide Studied by Infrared Absorption Spectroscopy. J. Phys. Chem. C 2011, 115 (40), 19761-19781.

161. Larciprete, R.; Fabris, S.; Sun, T.; Lacovig, P.; Baraldi, A.; Lizzit, S., Dual path mechanism in the thermal reduction of graphene oxide. J. Am. Chem. Soc. 2011, 133 (43), 17315-21.

162. Yang, H.; Cao, Y.; He, J.; Zhang, Y.; Jin, B.; Sun, J.-L.; Wang, Y.; Zhao, Z., Highly conductive free-standing reduced graphene oxide thin films for fast photoelectric devices. Carbon 2017, 115, 561-570.

163. Yang, D.; Velamakanni, A.; Bozoklu, G.; Park, S.; Stoller, M.; Piner, R. D.; Stankovich, S.; Jung, I.; Field, D. A.; Ventrice, C. A.; Ruoff, R. S., Chemical analysis of graphene oxide films after heat and chemical treatments by X-ray photoelectron and Micro-Raman spectroscopy. Carbon 2009, 47 (1), 145-152.

164. Nekahi, A.; Marashi, P. H.; Haghshenas, D., Transparent conductive thin film of ultra large reduced graphene oxide monolayers. Appl. Surf. Sci. 2014, 295, 59-65.

165.Eda, G.; Lin, Y.-Y.; Miller, S.; Chen, C.-W.; Su, W.-F.; Chhowalla, M., Transparent and conducting electrodes for organic electronics from reduced graphene oxide. Appl. Phys. Lett. 2008, 92 (23), 233305. 
166.Su, C.-Y.; Xu, Y.; Zhang, W.; Zhao, J.; Tang, X.; Tsai, C.-H.; Li, L.-J., Electrical and Spectroscopic Characterizations of Ultra-Large Reduced Graphene Oxide Monolayers. Chem. Mater. 2009, 21 (23), 5674-5680.

167. J. T. Robinson, F. K. P., E. S. Snow, Z. Wei, and P. E. Sheehan, Reduced Graphene Oxide Molecular Sensors. Nano Lett. 2008, 8 (10), 3137-3140.

168. Shin, H.-J.; Kim, K. K.; Benayad, A.; Yoon, S.-M.; Park, H. K.; Jung, I.-S.; Jin, M. H.; Jeong, H.-K.; Kim, J. M.; Choi, J.-Y.; Lee, Y. H., Efficient Reduction of Graphite Oxide by Sodium Borohydride and Its Effect on Electrical Conductance. Adv. Funct. Mater. 2009, 19 (12), 1987-1992.

169. Stankovich, S.; Dikin, D. A.; Piner, R. D.; Kohlhaas, K. A.; Kleinhammes, A.; Jia, Y.; Wu, Y.; Nguyen, S. T.; Ruoff, R. S., Synthesis of graphene-based nanosheets via chemical reduction of exfoliated graphite oxide. Carbon 2007, 45 (7), 1558-1565.

170. Park, S.; An, J.; Jung, I.; Piner, R. D.; An, S. J.; Li, X.; Velamakanni, A.; Ruoff, R. S., Colloidal suspensions of highly reduced graphene oxide in a wide variety of organic solvents. Nano Lett. 2009, 9, 1593-1597.

171. Gilje, S.; Han, S.; Wang, M.; Wang, K. L.; Kaner, R. B., A chemical route to graphene for device applications. Nano Lett. 2007, 7, 3394-3398.

172. Ambrosi, A.; Chua, C. K.; Bonanni, A.; Pumera, M., Lithium Aluminum Hydride as Reducing Agent for Chemically Reduced Graphene Oxides. Chem. Mater. 2012, 24 (12), 2292-2298.

173. Liu, H.; Zhang, L.; Guo, Y.; Cheng, C.; Yang, L.; Jiang, L.; Yu, G.; Hu, W.; Liu, Y.; Zhu, D., Reduction of graphene oxide to highly conductive graphene by Lawesson's reagent and its electrical applications. J. Mater. Chem. C 2013, 1 (18), 3104.

174.Zhou, X.; Zhang, J.; Wu, H.; Yang, H.; Zhang, J.; Guo, S., Reducing Graphene Oxide via Hydroxylamine: A Simple and Efficient Route to Graphene. J. Phys. Chem. C 2011, 115 (24), 11957-11961.

175. Chen, Y.; Zhang, X.; Yu, P.; Ma, Y., Stable dispersions of graphene and highly conducting graphene films: a new approach to creating colloids of graphene monolayers. Chem. Commun. 2009, (30), 4527.

176. Fernandez-Merino, M. J.; Guardia, L.; Paredes, J. I.; Villar-Rodil, S.; Solis-Fernandez, P.; Martinez-Alonso, A.; Tascon, J. M. D., Vitamin C is an ideal substitute for hydrazine in the reduction of graphene oxide suspensions. J. Phys. Chem. C 2010, 114, 6426-6432.

177. Chen, W.; Yan, L.; Bangal, P. R., Chemical reduction of graphene oxide to graphene by sulfur-containing compounds. J. Phys. Chem. C 2010, 114, 19885-19890.

178.Zhou, T.; Chen, F.; Liu, K.; Deng, H.; Zhang, Q.; Feng, J.; Fu, Q., A simple and efficient method to prepare graphene by reduction of graphite oxide with sodium hydrosulfite. Nanotechnology 2011, 22 (4), 045704.

179. Tu, Y.; Utsunomiya, T.; Ichii, T.; Sugimura, H., Enhancing the electrical conductivity of vacuum-ultraviolet-reduced graphene oxide by multilayered stacking. J. Vac. Sci. Technol. B 2017, 35 (3), 03D110.

180. Wang, D.-W.; Wu, K.-H.; Gentle, I. R.; Lu, G. Q., Anodic chlorine/nitrogen co-doping of reduced graphene oxide films at room temperature. Carbon 2012, 50 (9), 3333-3341.

181. Krishnamurthy, S.; Lightcap, I. V.; Kamat, P. V., Electron transfer between methyl viologen radicals and graphene oxide: Reduction, electron storage and discharge. J. Photoch. Photobio. A 2011, 221 (2-3), 214-219. 
182. Park, S.; Yoon, S. W.; Lee, K.-B.; Kim, D. J.; Jung, Y. H.; Do, Y.; Paik, H.-j.; Choi, I. S., Carbon Nanotubes as a Ligand in $\mathrm{Cp} 2 \mathrm{ZrCl} 2-\mathrm{Based}$ Ethylene Polymerization. Macromol. Rapid Comm. 2006, 27 (1), 47-50.

183. Park, S.; Choi, I. S., Production of Ultrahigh-Molecular-Weight Polyethylene/Pristine MWCNT Composites by Half-Titanocene Catalysts. Adv. Mater. 2009, 21 (8), 902-905.

184. Choi, B.; Lee, J.; Lee, S.; Ko, J. H.; Lee, K. S.; Oh, J.; Han, J.; Kim, Y. H.; Choi, I. S.; Park, S., Generation of ultra-high-molecular-weight polyethylene from metallocenes immobilized onto N-doped graphene nanoplatelets. Macromol. Rapid Commun. 2013, 34 (6), 533-8.

185. Connelly, N. G.; Geiger, W. E., Chemical redox agents for organometallic chemistry. Chem. Rev. 1996, 96, 877-910.

186. Nielson, R. M.; McManis, G. E.; Golovin, M. N.; Weaver, M. J., Solvent dynamical effects in electron transfer: comparisons of self-exchange kinetics for cobaltocenium-cobaltocene and related redox couples with theoretical predictions. J. Phys. Chem. 1988, 92, 3441-3450.

187. Nielson, R. M.; McManis, G. E.; Safford, L. K.; Weaver, M. J., Solvent and electrolyte effects on the kinetics of ferrocenium-ferrocene self-exchange: a reevaluation. J. Phys. Chem. 1989, 93, 2152-2157.

188. Banthorpe, D. V.; Charlwood, B. V.; Wasserman, A., Radical Anions Formed by Reaction of Cobaltocene with Protic Acids. J. Chem. Soc., Chem. Commun. 1972, 294-295.

189. Koelle, U.; Infelta, P. P.; Graetzel, M., Kinetics and Mechanism of the Reduction of Protons to Hydrogen by Cobaltocene. Inorg. Chem. 1988, 27, 879-883.

190. Chalkley, M. J.; Del Castillo, T. J.; Matson, B. D.; Roddy, J. P.; Peters, J. C., Catalytic N2to-NH3 Conversion by Fe at Lower Driving Force: A Proposed Role for MetalloceneMediated PCET. ACS Cent. Sci. 2017, 3 (3), 217-223.

191. Rountree, E. S.; Dempsey, J. L., Reactivity of Proton Sources with a Nickel Hydride Complex in Acetonitrile: Implications for the Study of Fuel-Forming Catalysts. Inorg. Chem. 2016, 55 (10), 5079-87.

192. Chen, J.; Zhang, Y.; Zhang, M.; Yao, B.; Li, Y.; Huang, L.; Li, C.; Shi, G., Water-enhanced oxidation of graphite to graphene oxide with controlled species of oxygenated groups. Chem. Sci. 2016, 7, 1874-1881.

193. McCreery, R. L., Structural Effects on Electron Transfer Kinetics. In Electroanal. Chem., Bard, A. J., Ed. Marcel Dekker, Inc.: New York, NY, 1966; Vol. 17, p 273.

194. Levi, G.; Senneca, O.; Causà, M.; Salatino, P.; Lacovig, P.; Lizzit, S., Probing the chemical nature of surface oxides during coal char oxidation by high-resolution XPS. Carbon 2015, 90, 181-196.

195. Cuesta, A.; Dhamelincourt, P.; Laureyns, J.; Martinez-Alonso, A.; Tascon, J. M. D., Raman microprobe studies on carbon materials. Carbon 1994, 32 (8), 1523-1532.

196. Eigler, S.; Hof, F.; Enzelberger-Heim, M.; Grimm, S.; Müller, P.; Hirsch, A., Statistical Raman Microscopy and Atomic Force Microscopy on Heterogeneous Graphene Obtained after Reduction of Graphene Oxide. J. Phys. Chem. C 2014, 118 (14), 7698-7704.

197. Gomez-Navarro, C.; Meyer, J. C.; Sundaram, R. S.; Chuvilin, A.; Kurasch, S.; Burghard, M.; Kern, K.; Kaiser, U., Atomic structure of reduced graphene oxide. Nano Lett. 2010, 10 (4), 1144-8.

198. Díez-Betriu, X.; Álvarez-García, S.; Botas, C.; Álvarez, P.; Sánchez-Marcos, J.; Prieto, C.; Menéndez, R.; de Andrés, A., Raman spectroscopy for the study of reduction mechanisms and optimization of conductivity in graphene oxide thin films. J. Mater. Chem. C 2013, 1 (41), 6905. 
199. Vollebregt, S.; Ishihara, R.; Tichelaar, F. D.; Hou, Y.; Beenakker, C. I. M., Influence of the growth temperature on the first and second-order Raman band ratios and widths of carbon nanotubes and fibers. Carbon 2012, 50 (10), 3542-3554.

200. Galus, Z.; Adams, R. N., The Anodic Oxidation of Triphenylmethane Dyes. J. Am. Chem. Soc. 1964, 86, 1666-1671.

201. Mernagh, T. P.; Cooney, R. P.; Johnson, R. A., Raman Spectra of Graphon Carbon Black. Carbon 1984, 22 (1), 39-42.

202. Basko, A. C. F. a. D. M., Raman spectroscopy as a versatile tool for studying the properties of graphene. Nat. Nanotechnol. 2013, 8.

203. Sakai, S.; Takayanagi, H.; Sumimoto, N.; Fukuzawa, S.-i.; Fujinami, T.; Saeki, H., Preparation and properties of surface-active organocobalt complexes having long-chain alkyl groups. Appl. Organomet. Chem. 1990, 4, 35-42.

204. Murr, N. E., Sels de cobalticinium. II*. Proprietes spectroscopiques et polarographiques de quelques derives du cobalticinium. J. Organomet. Chem. 1976, 112, 189-199.

205. Murr, N. E., Electrochemical reduction of substituted cobaltocinium cations in aqueous media. Transition Met. Chem. 1981, 6, 321-324.

206. Gaylord, N. G., Reduction with complex metal hydrides. J. Chem. Educ. 1957, 34, 367-374.

207. Cui, P.; Lee, J.; Hwang, E.; Lee, H., One-pot reduction of graphene oxide at subzero temperatures. Chem. Commun. 2011, 47 (45), 12370-2.

208. Mohan, V. B.; Brown, R.; Jayaraman, K.; Bhattacharyya, D., Characterisation of reduced graphene oxide: Effects of reduction variables on electrical conductivity. Mater. Sci. Eng. B 2015, 193, 49-60.

209. Wilson, A. D.; Newell, R. H.; McNevin, M. J.; Muckerman, J. T.; Rakowski DuBois, M.; DuBois, D. L., Hydrogen oxidation and production using nickel-based molecular catalysts with positioned proton relays. Journal of the American Chemical Society 2006, 128 (1), 358366.

210. Das, B.; Thapper, A.; Ott, S.; Colbran, S. B., Structural features of molecular electrocatalysts in multi-electron redox processes for renewable energy - recent advances. Sustainable Energy \& Fuels 2019, 3 (9), 2159-2175.

211. Matheu, R.; Garrido-Barros, P.; Gil-Sepulcre, M.; Ertem, M. Z.; Sala, X.; Gimbert-Suriñach, C.; Llobet, A., The development of molecular water oxidation catalysts. Nature Reviews Chemistry 2019, 3 (5), 331-341.

212. Gentil, S.; Serre, D.; Philouze, C.; Holzinger, M.; Thomas, F.; Le Goff, A., Electrocatalytic O2 Reduction at a Bio-inspired Mononuclear Copper Phenolato Complex Immobilized on a Carbon Nanotube Electrode. Angewandte Chemie International Edition 2016, 55 (7), 25172520.

213. Toma, F. M.; Sartorel, A.; Iurlo, M.; Carraro, M.; Rapino, S.; Hoober-Burkhardt, L.; Da Ros, T.; Marcaccio, M.; Scorrano, G.; Paolucci, F.; Bonchio, M.; Prato, M., Tailored Functionalization of Carbon Nanotubes for Electrocatalytic Water Splitting and Sustainable Energy Applications. ChemSusChem 2011, 4 (10), 1447-1451.

214. Das, A. K.; Engelhard, M. H.; Bullock, R. M.; Roberts, J. A., A hydrogen-evolving Ni (P2N2) 2 electrocatalyst covalently attached to a glassy carbon electrode: preparation, characterization, and catalysis. Comparisons with the homogeneous analogue. Inorganic chemistry 2014, 53 (13), 6875-6885. 
215. Yao, S. A.; Ruther, R. E.; Zhang, L.; Franking, R. A.; Hamers, R. J.; Berry, J. F., Covalent attachment of catalyst molecules to conductive diamond: $\mathrm{CO} 2$ reduction using "smart" electrodes. Journal of the American Chemical Society 2012, 134 (38), 15632-15635.

216. Kormányos, A.; Hossain, M. S.; Foss, F. W.; Janáky, C.; Rajeshwar, K., Electrocatalytic behavior of freely-diffusing and immobilized synthetic flavins in aqueous media. Catalysis Science \& Technology 2016, 6 (24), 8441-8448.

217.Eady, S. C.; MacInnes, M. M.; Lehnert, N., A Smorgasbord of Carbon: Electrochemical Analysis of Cobalt-Bis (benzenedithiolate) Complex Adsorption and Electrocatalytic Activity on Diverse Graphitic Supports. ACS applied materials \& interfaces 2016, 8 (36), 23624-23634.

218. Eady, S. C.; Peczonczyk, S. L.; Maldonado, S.; Lehnert, N., Facile heterogenization of a cobalt catalyst via graphene adsorption: robust and versatile dihydrogen production systems. Chemical Communications 2014, 50 (59), 8065-8068.

219.Zhou, X.; Wang, X.; Wang, B.; Chen, Z.; He, C.; Wu, Y., Preparation, characterization and NH3-sensing properties of reduced graphene oxide/copper phthalocyanine hybrid material. Sensors and actuators B: chemical 2014, 193, 340-348.

220. Yang, J.; Mu, D.; Gao, Y.; Tan, J.; Lu, A.; Ma, D., Cobalt phthalocyanine-graphene complex for electro-catalytic oxidation of dopamine. Journal of natural gas chemistry 2012, 21 (3), 265-269.

221. Andrieux, C.; Dumas-Bouchiat, J.; Saveant, J., Catalysis of electrochemical reactions at redox polymer electrodes: kinetic model for stationary voltammetric techniques. Journal of Electroanalytical Chemistry and Interfacial Electrochemistry 1982, 131, 1-35.

222. Costentin, C.; Saveant, J.-M., Cyclic voltammetry analysis of electrocatalytic films. The Journal of Physical Chemistry C 2015, 119 (22), 12174-12182.

223. Costentin, C.; Savéant, J.-M., Cyclic voltammetry of fast conducting electrocatalytic films. Physical Chemistry Chemical Physics 2015, 17 (29), 19350-19359.

224. Costentin, C.; Savéant, J. M., Cyclic voltammetry of electrocatalytic films: fast catalysis regimes. ChemElectroChem 2015, 2 (11), 1774-1784.

225. Oyama, N.; Anson, F. C., Catalysis of electrode processes by multiply-charged metal complexes electrostatically bound to polyelectrolyte coatings on graphite electrodes, and the use of polymer-coated rotating disk electrodes in diagnosing kinetic and conduction mechanisms. Analytical Chemistry 1980, 52 (8), 1192-1198.

226. Ikeda, T.; Leidner, C.; Murray, R. W., Kinetics of Outer-Sphere Electron Transfers Between Metal Complexes in Solutions and Polymeric Films on Modified Electrodes. Journal of the American Chemical Society 1981, 103 (25), 7422-7425.

227. Ohsaka, T.; Okajima, T.; Oyama, N., Electrode kinetics of metal complexes confined in electropolymerized poly (N, N-dialkyl substituted aniline) films on graphite surfaces. Journal of electroanalytical chemistry and interfacial electrochemistry 1986, 215 (1-2), 191-207.

228. Chiba, K.; Ohsaka, T.; Oyama, N., Electrode kinetics of electroactive electropolymerized polymers deposited on graphite electrode surfaces. Journal of electroanalytical chemistry and interfacial electrochemistry 1987, 217 (2), 239-251.

229. Kuo, K.-N.; Murray, R. W., Electrocatalysis with ferrocyanide electrostatically trapped in an alkylaminesiloxane polymer film on a Pt electrode. Journal of Electroanalytical Chemistry and Interfacial Electrochemistry 1982, 131, 37-59. 
230. Ikeda, T.; Schmehl, R.; Denisevich, P.; Willman, K.; Murray, R. W., Permeation of electroactive solutes through ultrathin polymeric films on electrode surfaces. Journal of the American Chemical Society 1982, 104 (10), 2683-2691.

231. Ciszewski, A.; Milczarek, G., Kinetics of electrocatalytic oxidation of formaldehyde on a nickel porphyrin-based glassy carbon electrode. Journal of Electroanalytical Chemistry 1999, 469 (1), 18-26.

232. Orchanian, N. M.; Hong, L. E.; Skrainka, J. A.; Esterhuizen, J. A.; Popov, D. A.; Marinescu, S. C., Surface-Immobilized Conjugated Polymers Incorporating Rhenium Bipyridine Motifs for Electrocatalytic and Photocatalytic CO2 Reduction. ACS Applied Energy Materials 2018, 2 (1), 110-123.

233. Coutanceau, C.; Croissant, M.; Napporn, T.; Lamy, C., Electrocatalytic reduction of dioxygen at platinum particles dispersed in a polyaniline film. Electrochimica Acta 2000, 46 (4), 579588.

234.Liu, Y.; Leung, K. Y.; Michaud, S. E.; Soucy, T. L.; McCrory, C. C., Controlled Substrate Transport to Electrocatalyst Active Sites for Enhanced Selectivity in the Carbon Dioxide Reduction Reaction. Comments on Inorganic Chemistry 2019, 39 (5), 242-269.

235. Liu, Y.; McCrory, C. C., Modulating the mechanism of electrocatalytic CO 2 reduction by cobalt phthalocyanine through polymer coordination and encapsulation. Nature communications 2019, 10 (1), 1-10.

236. Kulesza, P. J.; Karnicka, K.; Miecznikowski, K.; Chojak, M.; Kolary, A.; Barczuk, P. J.; Tsirlina, G.; Czerwinski, W., Network electrocatalytic films of conducting polymer-linked polyoxometallate-stabilized platinum nanoparticles. Electrochimica acta 2005, 50 (25-26), 5155-5162.

237. Jirkovský, J. S.; Halasa, M.; Schiffrin, D. J., Kinetics of electrocatalytic reduction of oxygen and hydrogen peroxide on dispersed gold nanoparticles. Physical Chemistry Chemical Physics 2010, 12 (28), 8042-8053.

238. Chen, X.; Xia, B.; He, P., Dynamics of the electrocatalytic oxidation of cysteine at Nafion film coated electrodes. Journal of electroanalytical chemistry and interfacial electrochemistry 1990, 281 (1-2), 185-198.

239. Abe, T.; Toda, G.; Tajiri, A.; Kaneko, M., Electrochemistry of ferric ruthenocyanide (Ruthenium Purple), and its electrocatalysis for proton reduction. Journal of Electroanalytical Chemistry 2001, 510 (1-2), 35-42.

240. Abe, T.; Kawai, N.; Tajiri, A.; Kaneko, M., Electrochemistry of ruthenium purple confined in a polymer matrix: Voltammetry, electrocatalysis for hydrogen evolution, and electrontransport characteristics. Bulletin of the Chemical Society of Japan 2003, 76 (3), 645-650.

241. Abe, T.; Fujita, T.; Sekimoto, K.; Tajiri, A.; Kaneko, M., Electrochemical hydrogen evolution by catalyst membrane composed of platinum, alkyl viologen and Nafion: consideration of the kinetically dominant factor based on understanding of electron-mediating ability of the viologen molecule. Journal of Molecular Catalysis A: Chemical 2003, 201 (1-2), 55-62.

242. Taguchi, F.; Abe, T.; Kaneko, M., New molecule-based catalyst to produce H2 by metalloporphine dispersed into polymer membrane. Journal of Molecular Catalysis A: Chemical 1999, 140 (1), 41-46.

243. Lee, I.; Joo, J. B.; Shokouhimehr, M., Graphene derivatives supported nanocatalysts for oxygen reduction reaction. Chinese Journal of Catalysis 2015, 36 (11), 1799-1810. 
244. Videla, A. H. M.; Ban, S.; Specchia, S.; Zhang, L.; Zhang, J., Non-noble Fe-NX electrocatalysts supported on the reduced graphene oxide for oxygen reduction reaction. Carbon 2014, 76, 386-400.

245.Lv, J.-J.; Li, S.-S.; Wang, A.-J.; Mei, L.-P.; Chen, J.-R.; Feng, J.-J., Monodisperse Au-Pd bimetallic alloyed nanoparticles supported on reduced graphene oxide with enhanced electrocatalytic activity towards oxygen reduction reaction. Electrochimica Acta 2014, 136, 521-528.

246.Xia, B. Y.; Wu, H. B.; Yan, Y.; Wang, H. B.; Wang, X., One-Pot Synthesis of Platinum Nanocubes on Reduced Graphene Oxide with Enhanced Electrocatalytic Activity. Small 2014, 10 (12), 2336-2339.

247. Huang, D.; Lu, J.; Li, S.; Luo, Y.; Zhao, C.; Hu, B.; Wang, M.; Shen, Y., Fabrication of cobalt porphyrin. Electrochemically reduced graphene oxide hybrid Films for electrocatalytic hydrogen evolution in aqueous solution. Langmuir 2014, 30 (23), 6990-6998.

248. McNamara, W. R.; Han, Z.; Yin, C.-J. M.; Brennessel, W. W.; Holland, P. L.; Eisenberg, R., Cobalt-dithiolene complexes for the photocatalytic and electrocatalytic reduction of protons in aqueous solutions. Proceedings of the National Academy of Sciences 2012, 109 (39), 15594-15599.

249. Achtyl, J. L.; Unocic, R. R.; Xu, L.; Cai, Y.; Raju, M.; Zhang, W.; Sacci, R. L.; Vlassiouk, I. V.; Fulvio, P. F.; Ganesh, P., Aqueous proton transfer across single-layer graphene. Nature communications 2015, 6 (1), 1-7.

250. NuLi, Y.; Yang, J.; Jiang, Z., Intercalation of lithium ions into bulk and powder highly oriented pyrolytic graphite. Journal of Physics and Chemistry of Solids 2006, 67 (4), 882-886.

251. Cho, Y. H.; Kim, H. W.; Lee, H. D.; Shin, J. E.; Yoo, B. M.; Park, H. B., Water and ion sorption, diffusion, and transport in graphene oxide membranes revisited. Journal of Membrane Science 2017, 544, 425-435.

252. Wang, Y.; Chen, Y.; Lacey, S. D.; Xu, L.; Xie, H.; Li, T.; Danner, V. A.; Hu, L., Reduced graphene oxide film with record-high conductivity and mobility. Materials Today 2018, 21 (2), 186-192.

253. Bird, C. L.; Kuhn, A. T., Electrochemistry of the viologens. Chemical Society Reviews 1981, $10(1), 49-82$.

254. Monk, P. M. S., The Viologens: Physicochemical Properties, Synthesis and Applications of the Salts of 4,4'-Bipyridine. John Wiley \& Sons Ltd.: West Sussex, England, 1998.

255. Striepe, L.; Baumgartner, T., Viologens and Their Application as Functional Materials. Chemistry 2017.

256. Liu, T.; Wei, X.; Nie, Z.; Sprenkle, V.; Wang, W., A Total Organic Aqueous Redox Flow Battery Employing a Low Cost and Sustainable Methyl Viologen Anolyte and 4-HO-TEMPO Catholyte. Advanced Energy Materials 2016, 6 (3).

257. Sen, S.; Saraidaridis, J.; Kim, S. Y.; Palmore, G. T., Viologens as charge carriers in a polymerbased battery anode. ACS Appl Mater Interfaces 2013, 5 (16), 7825-30.

258. Nagarjuna, G.; Hui, J.; Cheng, K. J.; Lichtenstein, T.; Shen, M.; Moore, J. S.; RodriguezLopez, J., Impact of redox-active polymer molecular weight on the electrochemical properties and transport across porous separators in nonaqueous solvents. J Am Chem Soc 2014, 136 (46), 16309-16.

259. Burgess, M.; Chénard, E.; Hernández-Burgos, K.; Nagarjuna, G.; Assary, R. S.; Hui, J.; Moore, J. S.; Rodríguez-López, J., Impact of Backbone Tether Length and Structure on the 
Electrochemical Performance of Viologen Redox Active Polymers. Chemistry of Materials 2016, 28 (20), 7362-7374.

260. Janoschka, T.; Friebe, C.; Hager, M. D.; Martin, N.; Schubert, U. S., An Approach Toward Replacing Vanadium: A Single Organic Molecule for the Anode and Cathode of an Aqueous Redox-Flow Battery. ChemistryOpen 2017, 6 (2), 216-220.

261. Mortimer, R. J., Organic electrochromic materials. Electrochimica Acta 1999, 44 (18), 29712981.

262. Mortimer, R. J., Electrochromic Materials. Annual Review of Materials Research 2011, 41 (1), 241-268.

263. Jeon, W. S.; Ziganshina, A. Y.; Lee, J. W.; Ko, Y. H.; Kang, J. K.; Lee, C.; Kim, K., A [2] Pseudorotaxane-Based Molecular Machine: Reversible Formation of a Molecular Loop Driven by Electrochemical and Photochemical Stimuli. Angewandte Chemie 2003, 115 (34), 4231-4234.

264. Song, Y.; Huang, X.; Hua, H.; Wang, Q., The synthesis of a rigid conjugated viologen and its cucurbituril pseudorotaxanes. Dyes and Pigments 2017, 137, 229-235.

265. Wessels, H. R.; Slebodnick, C.; Gibson, H. W., Viologen-Based Rotaxanes from Dibenzo30-crown-10. Journal of the American Chemical Society 2018, 140 (23), 7358-7370.

266. Zhang, B.; Dong, Y.; Li, J.; Yu, Y.; Li, C.; Cao, L., Pseudo[n,m]rotaxanes of Cucurbit[7/8]uril and Viologen-Naphthalene Derivative: A Precise Definition of Rotaxane. Chinese Journal of Chemistry 2019, 37 (3), 269-275.

267. Thorneley, R. N. F., A convenient electrochemical preparation of reduced methyl viologen and a kinetic study of the reaction with oxygen using an anaerobic stopped-flow apparatus. Biochimica et Biophysica Acta 1974, 333, 487-496.

268. Leest, R. E. V. D., The coulometric determination of oxygen with the electolytically generated viologen radical-cation. Electroanalytical Chemistry and Interfacial Electrochemistry 1973, 43, 251-255.

269. Farrington, J. A.; Ebert, M.; Land, E. J., Bipyridylium quaternary salts and related compounds. Part 6.-Pulse radiolysis studies of the reaction of paraquat radical analogues with oxygen. Journal of the Chemical Society, Faraday Transactions 1: Physical Chemistry in Condensed Phases 1978, 74, 665-675.

270. Hoshino, K.; Sasaki, H.; Suga, K.; Saji, T., Partition parameters for three redox forms of viologen in a nonionic micellar solution. Bull. Chem. Soc. Jpn. 1987, 60, 1521-1522.

271. Kaifer, A. E.; Bard, A. J., Micellar effects on the reductive electrochemistry of methylviologen. The Journal of Physical Chemistry 1985, 89 (22), 4876-4880.

272. Kim, H.-J.; Jeon, W. S.; Young, H. K.; Kim, K., Inclusion of methylviologen in cucurbit[7]uril. PNAS 2002, 99 (8), 5007-5011.

273. Kostela, J.; Elmgren, M.; Hansson, P.; Almgren, M., Electrochemical properties of an amphiphilic viologen in differently charged micelles. Journal of Electroanalytical Chemistry 2002, 536, 97-107.

274.Lee, C.-W.; M.-K., O.; Jang, J.-M., Reduction potentials of N-hexadecyl-N'-methyl viologen $(2+/+)$ solubilized in cationic, nonionic, and anionic micelles. Langmuir 1993, 9, 1934-1936.

275.Park, J. W.; Ko, S. H.; Park, J.-Y., Electrochemical studies of viologens in homogeneous aqueous and sodium dodecyl sulfate micellar solutions. Bull. Korean Chem. Soc. 1992, 13, 259-265. 
276. Kaifer, A. E.; Li, W.; Yi, S., Cucurbiturils as Versatile Receptors for Redox Active Substrates. Israel Journal of Chemistry 2011, 51 (5-6), 496-505.

277. Pospisil, L.; Fuoco, R.; Papoff, P., Electrocatalytic reduction of oxygen by cation radicals of methyl viologen or diquat complexes with macrocyclic crown ethers. J. Electroanal. Chem. 1988, 256, 83-93.

278. Willman, K. W.; Murray, R. W., Viologen homopolymer, polymer mixture and polymer bilayer films on electrodes: Electropolymerization, electrolysis, spectroelectrochemistry, trace analysis and photoreduction. Journal of Electroanalytical Chemistry and Interfacial Electrochemistry 1982, 133 (2), 211-231.

279. Bookbinder, D. C.; Wrighton, M. S., Electrochromic polymers covalently anchored to electrode surfaces. Optical and electrochemical properties of a viologen-based polymer. Journal of the Electrochemical Society 1983, 130 (5), 1080-1087.

280. Liu, X.; Neoh, K. G.; Kang, E. T., Redox-Sensitive Microporous Membranes Prepared from Poly(vinylidene fluoride) Grafted with Viologen-Containing Polymer Side Chains. Macromolecules 2003, 36 (22), 8361-8367.

281. Kepley, L. J.; Bard, A. J., Polymer Films on Electrodes XXVII. Electrochemical and Ellipsometric Measurements of a Viologen-Siloxane Polymer Film: Deposition, Solvent Swelling, Oxidation-State-Dependent Thickness, and Charge Transport. Journal of The Electrochemical Society 1995, 142 (12), 4129-4138.

282. Mortimer, R. J.; Dyer, A. L.; Reynolds, J. R., Electrochromic organic and polymeric materials for display applications. Displays 2006, 27 (1), 2-18.

283. Nakahira, T.; Graetzel, M., Fast electron storage with colloidal semiconductors functionalized with polymeric viologen. The Journal of Physical Chemistry 1984, 88 (18), 4006-4010.

284. Sato, H.; Tamamura, T., Polymer effect in electrochromic behavior of oligomeric viologens. Journal of Applied Polymer Science 1979, 24 (10), 2075-2085.

285. Oyama, N.; Ohsaka, T.; Yamamoto, H.; Kaneko, M., Charge-transfer reactions in pendant viologen polymers coated on graphite electrodes and at electrode/pendant viologen polymer film interfaces. The Journal of Physical Chemistry 1986, 90 (16), 3850-3856.

286. Dalton, E.; Murray, R. W., Viologen (2+/1+) and viologen (1+/0) electron-self-exchange reactions in a redox polymer. The Journal of Physical Chemistry 1991, 95 (16), 6383-6389.

287. Simon, M. S.; Moore, P. T., Novel polyviologens: Photochromic redox polymers with filmforming properties. Journal of Polymer Science: Polymer Chemistry Edition 1975, 13 (1), 116.

288. Factor, A.; Heinsohn, G., Polyviologens-a novel class of cationic polyelectrolyte redox polymers. Journal of Polymer Science Part B: Polymer Letters 1971, 9 (4), 289-295.

289. Tomokazu, M.; Tetsuya, K.; Uichi, A.; Tetsuo, O., INCLUSION, SOLUBILIZATION, AND STABILIZATION OF TWO-ELECTRON REDUCED SPECIES OF METHYL VIOLOGEN BY CYCLODEXTRINS. Chemistry Letters 1985, 14 (12), 1825-1828.

290. Annibaldi, V.; Breslin, C. B., Electrochemistry of viologens at polypyrrole doped with sulfonated $\beta$-cyclodextrin. Journal of Electroanalytical Chemistry 2019, 832, 399-407.

291. Ong, W.; Gómez-Kaifer, M.; Kaifer, A. E., Cucurbit[7]uril: A Very Effective Host for Viologens and Their Cation Radicals. Organic Letters 2002, 4 (10), 1791-1794.

292. Sun, S.; Zhang, R.; Andersson, S.; Pan, J.; Zou, D.; Åkermark, B.; Sun, L., Host-Guest Chemistry and Light Driven Molecular Lock of Ru(bpy)3-Viologen with Cucurbit[7-8] urils. The Journal of Physical Chemistry B 2007, 111 (47), 13357-13363. 
293. Buck, A. T.; Paletta, J. T.; Khindurangala, S. A.; Beck, C. L.; Winter, A. H., A noncovalently reversible paramagnetic switch in water. Journal of the American Chemical Society 2013, 135 (29), 10594-10597.

294. El-Barghouthi, M. I.; Assaf, K. I.; Rawashdeh, A. M. M., Molecular dynamics of methyl viologen-cucurbit[n] uril complexes in aqueous solution. J. Chem. Theory Comput. 2010, 6, 984-992.

295. Blandamer, M. J.; Brivati, J. A.; Fox, M. F.; Symons, M. C. R.; Verman, G. S. P., Waterinduced dimerization of various dye and related paramagnetic ions studied by optical and electron spin resonance spectroscopy. Trans. Faraday Soc. 1967, 63, 1850-1857.

296. Neta, P.; Richoux, M.-C.; Harriman, A., Intramolecular association of covalently linked viologen radicals. Journal of the Chemical Society, Faraday Transactions 2: Molecular and Chemical Physics 1985, 81 (9), 1427-1443.

297. Lü, J.-M.; Rosokha, S. V.; Kochi, J. K., Stable (long-bonded) dimers via the quantitative selfassociation of different cationic, anionic, and uncharged $\pi$-radicals: Structures, energetics, and optical transitions. Journal of the American Chemical Society 2003, 125 (40), 12161-12171.

298. Deronzier, A.; Galland, B.; Vieira, M., Electrochemical behavior of some paraquat dimer molecules a potential direct bielectronic mediating system. Nouv. J. Chim. 1982, 6, 97-100.

299. Geraskina, M. R.; Dutton, A. S.; Juetten, M. J.; Wood, S. A.; Winter, A. H., The Viologen Cation Radical Pimer: A Case of Dispersion-Driven Bonding. Angewandte Chemie 2017, 129 (32), 9563-9567.

300. Neta, P.; Richoux, M.-C.; Harriman, A., Intramolecular Association of Covalently Linked Viologen Radicals. J. Chem. Soc., Faraday Trans. 2 1985, 81, 1427-1443.

301. Enea, O.; Crouigneau, P.; Moser, J.; Graetzel, M.; Huenig, S. On the pimerization of bridged viologen radicals photoproduced at the surface of illuminated titanium(IV) oxide sols, p. 26771-267-71. (accessed 1991).

302. Masaoki, F.; Shun-ichi, N., Photoreduction of Bisviologen Compounds, Viologen-(CH2)nViologen, by 2-Propanol. Bulletin of the Chemical Society of Japan 1982, 55 (2), 513-516.

303. Richardson, D. E.; Taube, H., Determination of E20-E10 in multistep charge transfer by stationary-electrode pulse and cyclic voltammetry: application to binuclear ruthenium ammines. Inorganic Chemistry 1981, 20 (4), 1278-1285.

304. Peerannawar, S. R.; Gobre, V. V.; Gejji, S. P., Binding of viologen derivatives to cucurbit [8] uril. Computational and Theoretical Chemistry 2012, 983, 16-24.

305. Mizuguchi, J.; Karfunkel, H., Semi-Empirical Calculations on the Optical Absorption of Methylviologen and p-Cyanophenylviologen in Different Oxidation States. Berichte der Bunsengesellschaft für physikalische Chemie 1993, 97 (11), 1466-1472.

306. Lee, C.; Lee, Y. M.; Moon, M. S.; Park, S. H.; Park, J. W.; Kim, K. G.; Jeon, S.-J., UV-visNIR and Raman spectroelectrochemical studies on viologen cation radicals: evidence for the presence of various types of aggregate species. Journal of Electroanalytical Chemistry 1996, 416 (1), 139-144.

307. Odell, B.; Reddington, M. V.; Slawin, A. M. Z.; Spencer, N.; Stoddart, J. F.; Williams, D. J., Cyclobis(paraquat-p-phenylene). A Tetracationic Multipurpose Receptor. Angewandte Chemie International Edition in English 1988, 27 (11), 1547-1550.

308. Rauwolf, C.; Straßner, T., Modeling of Selforganizing Systems. Molecular modeling annual 1997, 3 (1), 1-16.

309. Geuder, W.; Hünig, S.; Suchy, A., Single and double bridged viologenes and intramolecular pimerization of their cation radicals. Tetrahedron 1986, 42 (6), 1665-1677. 
310. Enea, O.; Crouigneau, P.; Moser, J.; Grätzel, M.; Huenig, S., On the pimerization of bridged viologen radicals photoproduced at the surface of illuminated titanium (IV) oxide sols. New journal of chemistry 1991, 15 (ARTICLE), 267-71.

311.Zhang, D. W.; Tian, J.; Chen, L.; Zhang, L.; Li, Z. T., Dimerization of Conjugated Radical Cations: An Emerging Non-Covalent Interaction for Self-Assembly. Chemistry-An Asian Journal 2015, 10 (1), 56-68.

312. Huang, J.; Kertesz, M., Intermolecular covalent $\pi-\pi$ bonding interaction indicated by bond distances, energy bands, and magnetism in biphenalenyl biradicaloid molecular crystal. Journal of the American Chemical Society 2007, 129 (6), 1634-1643.

313. Goto, K.; Kubo, T.; Yamamoto, K.; Nakasuji, K.; Sato, K.; Shiomi, D.; Takui, T.; Kubota, M.; Kobayashi, T.; Yakusi, K., A stable neutral hydrocarbon radical: Synthesis, crystal structure, and physical properties of 2, 5, 8-tri-tert-butyl-phenalenyl. Journal of the American Chemical Society 1999, 121 (7), 1619-1620.

314. Fukui, K.; Sato, K.; Shiomi, D.; Takui, T.; Itoh, K.; Gotoh, K.; Kubo, T.; Yamamoto, K.; Nakasuji, K.; Naito, A., Electronic structure of a stable phenalenyl radical in crystalline state as studied by SQUID measurements, cw-ESR, and 13C CP/MAS NMR spectroscopy. Synthetic metals 1999, 103 (1-3), 2257-2258.

315. Kosower, E. M.; Cotter, J. L., Stable Free Radicals. II. The Reduction of 1-Methyl-4cyanopyridinium Ion to Methylviologen Cation Radical. Journal of the American Chemical Society 1964, 86 (24), 5524-5527.

316.Zafra, J. L.; Qiu, L.; Yanai, N.; Mori, T.; Nakano, M.; Alvarez, M. P.; Navarrete, J. T. L.; Gómez-García, C. J.; Kertesz, M.; Takimiya, K.; Casado, J., Reversible Dimerization and Polymerization of a Janus Diradical To Produce Labile $\mathrm{C}-\mathrm{C}$ Bonds and Large Chromic Effects. Angewandte Chemie International Edition 2016, 55 (47), 14563-14568.

317. Preuss, K. E., Pancake bonds: $\pi$-Stacked dimers of organic and light-atom radicals. Polyhedron 2014, 79, 1-15.

318. Miller, L. L.; Mann, K. R., $\pi$-Dimers and $\pi$-Stacks in Solution and in Conducting Polymers. Accounts of Chemical Research 1996, 29 (9), 417-423.

319. Kertesz, M., Pancake Bonding: An Unusual Pi-Stacking Interaction. Chemistry - A European Journal 2019, 25 (2), 400-416.

320. Trabolsi, A.; Khashab, N.; Fahrenbach, A. C.; Friedman, D. C.; Colvin, M. T.; Cotí, K. K.; Benítez, D.; Tkatchouk, E.; Olsen, J.-C.; Belowich, M. E., Radically enhanced molecular recognition. Nature chemistry 2010, 2 (1), 42.

321. Collier, C. P.; Mattersteig, G.; Wong, E. W.; Luo, Y.; Beverly, K.; Sampaio, J.; Raymo, F. M.; Stoddart, J. F.; Heath, J. R., A [2] catenane-based solid state electronically reconfigurable switch. Science 2000, 289 (5482), 1172-1175.

322. Geuder, W.; Hünig, S.; Suchy, A., Phane mit zwei 4,4'-Bipyridiniumeinheiten - eine neue Verbindungsklasse. Angewandte Chemie 1983, 95 (6), 501-502.

323. Moser, J.; Graetzel, M., Light-induced electron transfer in colloidal semiconductor dispersions: single vs. dielectronic reduction of acceptors by conduction-band electrons. Journal of the American Chemical Society 1983, 105 (22), 6547-6555.

324. Sun, X.; Yang, Y.-K., Photochromism of double-bridged viologens in a polar polymer matrix. Journal of the Chemical Society, Perkin Transactions 2 1996, (2), 225-228.

325. Yang, Y.-K.; Sun, X., PHOTOREDUCTION OF DOUBLE-BRIDGED VIOLOGEN POLYMER MATRIX FILM. Journal of Photopolymer Science and Technology 1994, 7 (1), 193-198. 
326. Katsuki, T.; Sharpless, K. B., The first practical method for asymmetric epoxidation. Journal of the American Chemical Society 1980, 102 (18), 5974-5976.

327. MacInnes, M. M.; Hlynchuk, S.; Acharya, S.; Lehnert, N.; Maldonado, S., Reduction of Graphene Oxide Thin Films by Cobaltocene and Decamethylcobaltocene. ACS applied materials \& interfaces 2017, 10 (2), 2004-2015.

328. Rozlosnik, N.; Gerstenberg, M. C.; Larsen, N. B., Effect of Solvents and Concentration on the Formation of a Self-Assembled Monolayer of Octadecylsiloxane on Silicon (001). Langmuir 2003, 19 (4), 1182-1188.

329. Wang, Y.; Lieberman, M., Growth of Ultrasmooth Octadecyltrichlorosilane Self-Assembled Monolayers on SiO2. Langmuir 2003, 19 (4), 1159-1167.

330. Ulman, A., Formation and structure of self-assembled monolayers. Chemical reviews 1996, 96 (4), 1533-1554.

331. Eigler, S.; Hu, Y.; Ishii, Y.; Hirsch, A., Controlled functionalization of graphene oxide with sodium azide. Nanoscale 2013, 5 (24), 12136-12139.

332. Bell, C. A.; Jia, Z.; Perrier, S.; Monteiro, M. J., Modulating catalytic activity of polymerbased cuAAC "click” reactions. Journal of Polymer Science Part A: Polymer Chemistry 2011, 49 (21), 4539-4548.

333. Mukherjee, J.; Peczonczyk, S.; Maldonado, S., Wet chemical functionalization of III- V semiconductor surfaces: Alkylation of gallium phosphide using a grignard reaction sequence. Langmuir 2010, 26 (13), 10890-10896.

334. Cote, L. J.; Kim, J.; Zhang, Z.; Sun, C.; Huang, J., Tunable assembly of graphene oxide surfactant sheets: wrinkles, overlaps and impacts on thin film properties. Soft Matter 2010, 6 (24), 6096-6101.

335. Taylor, A. P.; Velásquez-García, L. F., Electrospray-printed nanostructured graphene oxide gas sensors. Nanotechnology 2015, 26 (50), 505301.

336. Shao, J.-J.; Lv, W.; Yang, Q.-H., Self-Assembly of Graphene Oxide at Interfaces. Advanced Materials 2014, 26 (32), 5586-5612.

337. Gerischer, H., Photodecomposition of semiconductors thermodynamics, kinetics and application to solar cells. Faraday Discussions of the Chemical Society 1980, 70, 137-151.

338. Sun, K.; Shen, S.; Liang, Y.; Burrows, P. E.; Mao, S. S.; Wang, D., Enabling silicon for solarfuel production. Chemical reviews 2014, 114 (17), 8662-8719.

339. DeBenedetti, W. J. I.; Chabal, Y. J., Functionalization of oxide-free silicon surfaces. Journal of Vacuum Science \& Technology A 2013, 31 (5), 050826.

340. Thissen, P.; Seitz, O.; Chabal, Y. J., Wet chemical surface functionalization of oxide-free silicon. Progress in surface science 2012, 87 (9-12), 272-290.

341. Liu, R.; Zheng, Z.; Spurgeon, J.; Yang, X., Enhanced photoelectrochemical water-splitting performance of semiconductors by surface passivation layers. Energy \& Environmental Science 2014, 7 (8), 2504-2517.

342. Wang, Y.; Li, Z.; Wang, J.; Li, J.; Lin, Y., Graphene and graphene oxide: biofunctionalization and applications in biotechnology. Trends in biotechnology 2011, 29 (5), 205-212.

343. Iqbal, M.; Li, C.; Jiang, B.; Hossain, M. S. A.; Islam, M. T.; Henzie, J.; Yamauchi, Y., Tethering mesoporous $\mathrm{Pd}$ nanoparticles to reduced graphene oxide sheets forms highly efficient electrooxidation catalysts. Journal of Materials Chemistry A 2017, 5 (40), 2124921256. 
344. Hu, S. H.; Chen, Y. W.; Hung, W. T.; Chen, I. W.; Chen, S. Y., Quantum-dot-tagged reduced graphene oxide nanocomposites for bright fluorescence bioimaging and photothermal therapy monitored in situ. Advanced materials 2012, 24 (13), 1748-1754.

345. Xu, Y.-y.; Niu, X.-y.; Dong, Y.-1; Zhang, H.-g.; Li, X.; Chen, H.-1.; Chen, X.-g., Preparation and characterization of open-tubular capillary column modified with graphene oxide nanosheets for the separation of small organic molecules. Journal of Chromatography A 2013, $1284,180-187$.

346. Kulesza, P. J.; Grzybowska, B.; Malik, M. A.; Galkowski, M. T., Tungsten oxides as active supports for highly dispersed platinum microcenters: electrocatalytic reactivity toward reduction of hydrogen peroxide and oxygen. Journal of The Electrochemical Society 1997, 144 (6), 1911. 